\title{
Fe(III)-catalyzed $N$-amidomethylation of secondary and primary anilines with TosMIC
}

\author{
Yigao Li, Xiaohuang Ren, Yi Chen, Xinju Zhu, Xin-Qi Hao, Mao-Ping Song* \\ College of Chemistry, Zhengzhou University, No. 100 of Science Road, Zhengzhou, 450001, P. \\ R. China \\ Email: zhuxinju@zzu.edu.cn; mpsong@zzu.edu.cn
}




\section{Table of Contents}

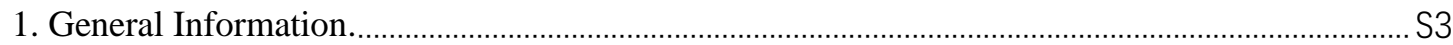

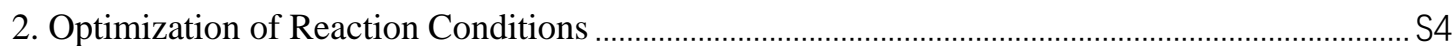

3. General Procedure for the Preparation of 3, 5-10 ..................................................................... S7

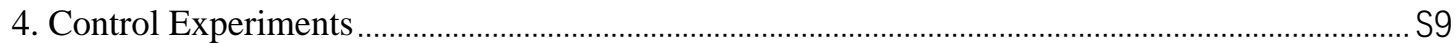

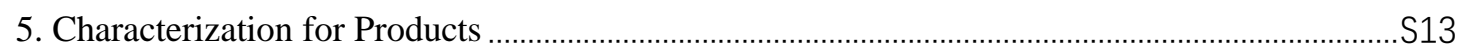

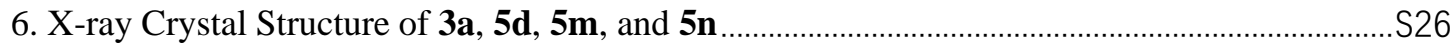

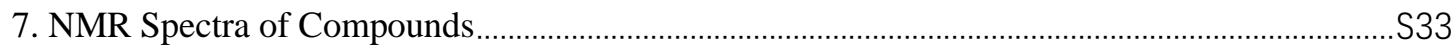




\section{General Information.}

Unless otherwise indicated, all the starting materials and reagents were commercially available and used without further purification. Aniline derivatives $\mathbf{4 b}-\mathbf{d}^{\mathbf{1}}, \mathbf{4} \mathbf{g}-\mathbf{h}^{1}$ and TosMIC derivatives $\mathbf{2 a - \mathbf { e } ^ { 2 }}$ are known compounds and synthesized according to previously reported methods. Melting points were determined on a melting point apparatus. Flash column chromatography was performed using 200-300 mesh silica gel. Analytical and preparative thin-layer chromatography (TLC) plates coated with commercial silica gel GF254 were used to monitor the reactions and purify products. ${ }^{1} \mathrm{H}$, ${ }^{13} \mathrm{C}\left\{{ }^{1} \mathrm{H}\right\}$, and ${ }^{19} \mathrm{~F}$ NMR spectra were recorded on Bruker DPX 400 or Bruker DPX 600 instruments using TMS as an internal standard. Data are reported as follows: chemical shift ( $\delta \mathrm{ppm})$, multiplicity ( $\mathrm{s}=$ singlet, $\mathrm{d}=$ doublet, $\mathrm{t}=$ triplet, $\mathrm{m}=$ multiplet, $\mathrm{br}=$ broad, $\mathrm{dd}=$ double doublet, $\mathrm{dt}=$ double triplet), integration, and coupling constants $(J)$ in hertz $(\mathrm{Hz})$. HRMS were determined on a Q-TOF micro MS/MS system ESI spectrometer. The structure of products 3a (CCDC file Number 2109915), 5d (CCDC file Number2109916), 5m (CCDC file Number 2109913), and 5n (CCDC file Number 2109914) were further confirmed by X-ray diffraction collected on a diffractometer with graphitemono-chromated Mo $\mathrm{K} \alpha$ radiation.

Warning: Special precaution should be taken when performing workups since cyanide waste may be generated!!! 


\section{Optimization of Reaction Conditions}

Table S1. Optimization of solvent and dosages of $2 \mathrm{a}^{a}$

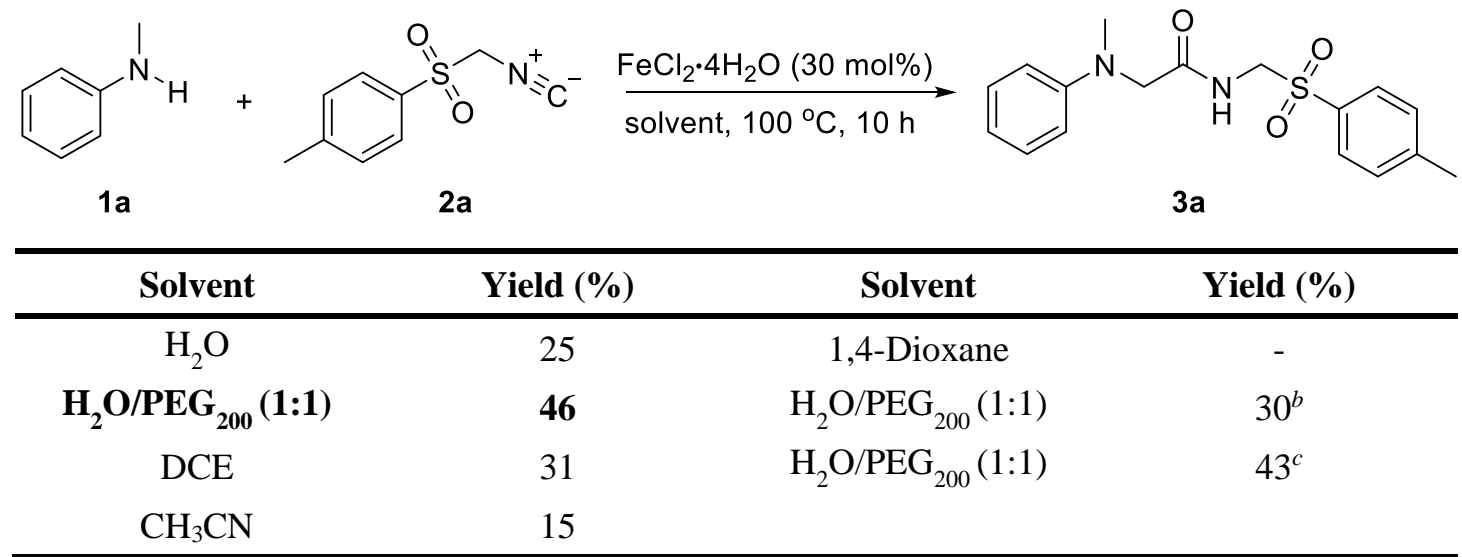

${ }^{a}$ Reaction conditions: 1a $(0.2 \mathrm{mmol}), \mathbf{2 a}(0.6 \mathrm{mmol}), \mathrm{FeCl}_{2} \cdot 4 \mathrm{H}_{2} \mathrm{O}(30 \mathrm{~mol} \%)$, solvent $(2 \mathrm{~mL})$, $100{ }^{\circ} \mathrm{C}, 10 \mathrm{~h}$, under air. ${ }^{b} \mathbf{2 a}(0.4 \mathrm{mmol}),{ }^{c} \mathbf{2} \mathbf{a}(0.8 \mathrm{mmol})$.

Table S2. Optimization of catalysts ${ }^{a}$

\begin{tabular}{cccc} 
1a & Yatalyst & Yield (\%) \\
\hline Catalyst & 46 & $\mathrm{Co}(\mathrm{acac})_{2}$ & 23 \\
\hline $\mathrm{FeCl}_{2} \cdot 4 \mathrm{H}_{2} \mathrm{O}$ & 44 & $\mathrm{Co}(\mathrm{acac})_{3}$ & 40 \\
$\mathrm{FeSO}_{4} \cdot 7 \mathrm{H}_{2} \mathrm{O}$ & $\mathbf{5 2}$ & $\mathrm{CuI}$ & 17 \\
$\mathbf{F e}(\mathbf{a c a c})_{3}$ & 46 & $\mathrm{Cu}(\mathrm{OAc})_{2} \cdot \mathrm{H}_{2} \mathrm{O}$ & 7 \\
$\mathrm{Fe}\left(\mathrm{ClO}_{4}\right)_{2}$ & 36 & $\mathrm{Cu}(\mathrm{acac})_{2}$ & 17 \\
$\mathrm{NiCl}_{2}$ & 32 & - & 38 \\
$\mathrm{CoCl}_{2} \cdot 6 \mathrm{H}_{2} \mathrm{O}$ & & & -
\end{tabular}

${ }^{a}$ Reaction conditions: 1a $(0.2 \mathrm{mmol}), \mathbf{2 a}(0.6 \mathrm{mmol})$, catalyst $(30 \mathrm{~mol} \%), \mathrm{H}_{2} \mathrm{O} / \mathrm{PEG}_{200}(1: 1)(2 \mathrm{~mL})$, $100{ }^{\circ} \mathrm{C}, 10 \mathrm{~h}$, under air. 
Table S3. Optimization of additive without catalyst ${ }^{a}$

\begin{tabular}{|c|c|c|c|}
\hline 1a & & & $3 a$ \\
\hline Additive & Yield (\%) & Additive & Yield (\%) \\
\hline - & 38 & $\mathrm{~K}_{3} \mathrm{PO}_{4} \cdot 3 \mathrm{H}_{2} \mathrm{O}$ & 15 \\
\hline $\mathrm{NaOCH}_{3}$ & 21 & $\mathrm{CH}_{3} \mathrm{COOH}$ & 39 \\
\hline $\mathrm{KO}^{t} \mathrm{Bu}$ & 33 & $\mathrm{MesCOOH}$ & 40 \\
\hline $\mathrm{NaOH}$ & 35 & 1-AdCOOH & 39 \\
\hline $\mathrm{CsCO}_{3}$ & 11 & $\mathrm{CF}_{3} \mathrm{COOH}$ & 20 \\
\hline
\end{tabular}

${ }^{a}$ Reaction conditions: 1a $(0.2 \mathrm{mmol}), \mathbf{2 a}(0.6 \mathrm{mmol})$, additive (1 equiv), $\mathrm{H}_{2} \mathrm{O} / \mathrm{PEG}_{200}(1: 1)(2 \mathrm{~mL})$, $100^{\circ} \mathrm{C}, 10 \mathrm{~h}$, under air.

Table S4. Optimization of temperature ${ }^{a}$

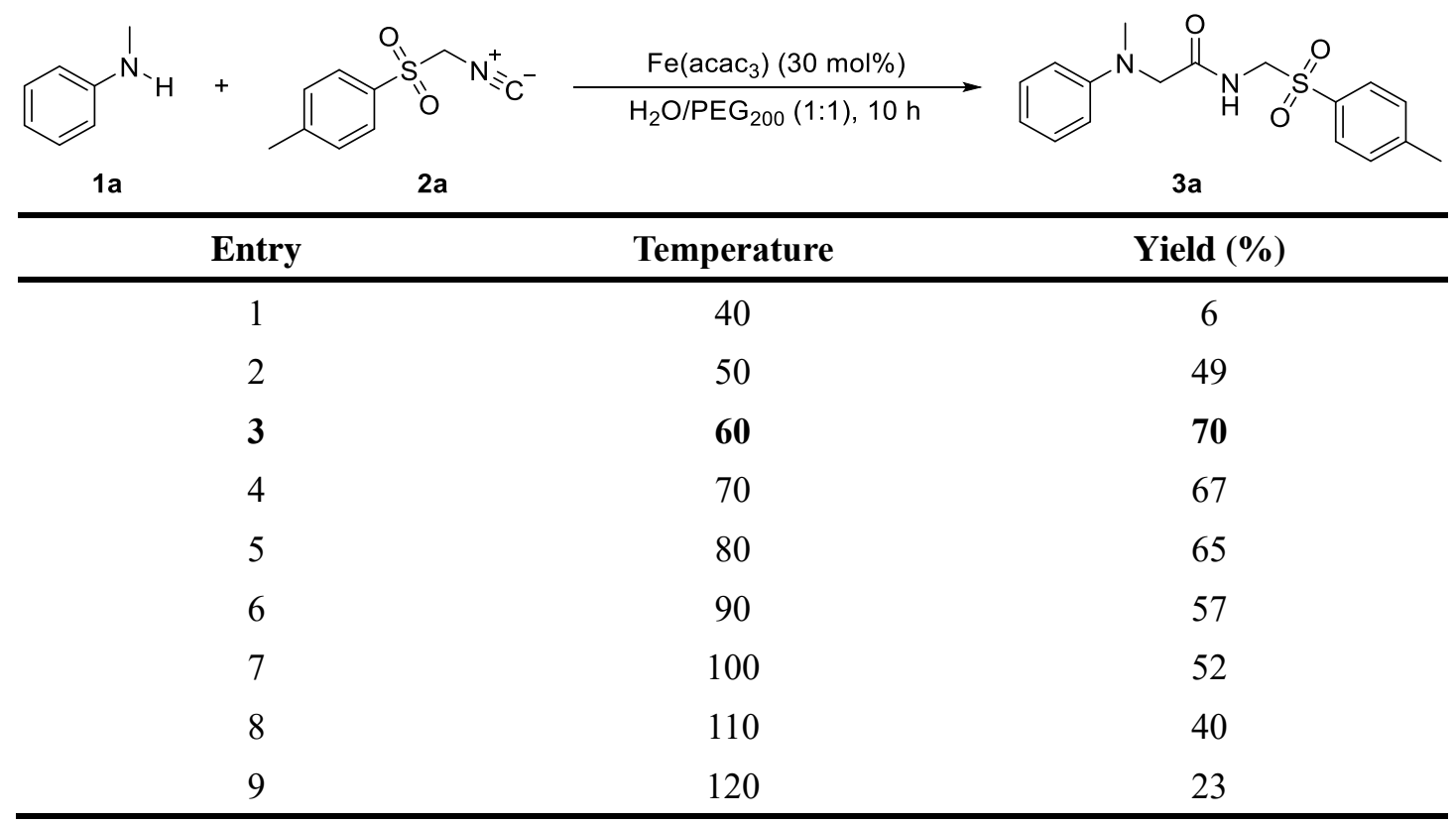

${ }^{a}$ Reaction conditions: 1a $(0.2 \mathrm{mmol}), \mathbf{2 a}(0.6 \mathrm{mmol}), \mathrm{Fe}(\mathrm{acac})_{3}(30 \mathrm{~mol} \%), \mathrm{H}_{2} \mathrm{O} / \mathrm{PEG}_{200}(1: 1)(2$ $\mathrm{mL}), 10 \mathrm{~h}$, under air. 
Table S5. Optimization of dosages of $\mathrm{Fe}(\mathrm{acac})_{3}{ }^{a}$

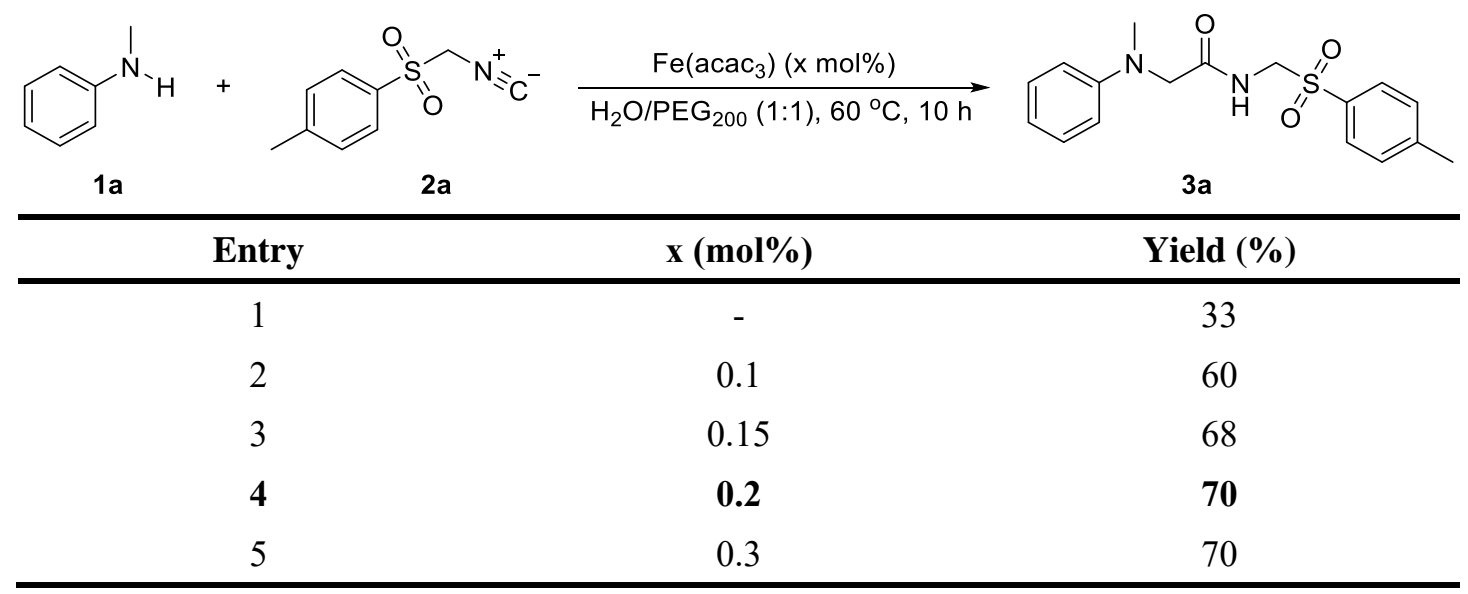

${ }^{a}$ Reaction conditions: 1a $(0.2 \mathrm{mmol}), \mathbf{2 a}(0.6 \mathrm{mmol}), \mathrm{Fe}(\mathrm{acac})_{3}, \mathrm{H}_{2} \mathrm{O} / \mathrm{PEG}_{200}(1: 1)(2 \mathrm{~mL}), 60^{\circ} \mathrm{C}, 10$ $\mathrm{h}$, under air.

Table S6. Optimization of time ${ }^{a}$

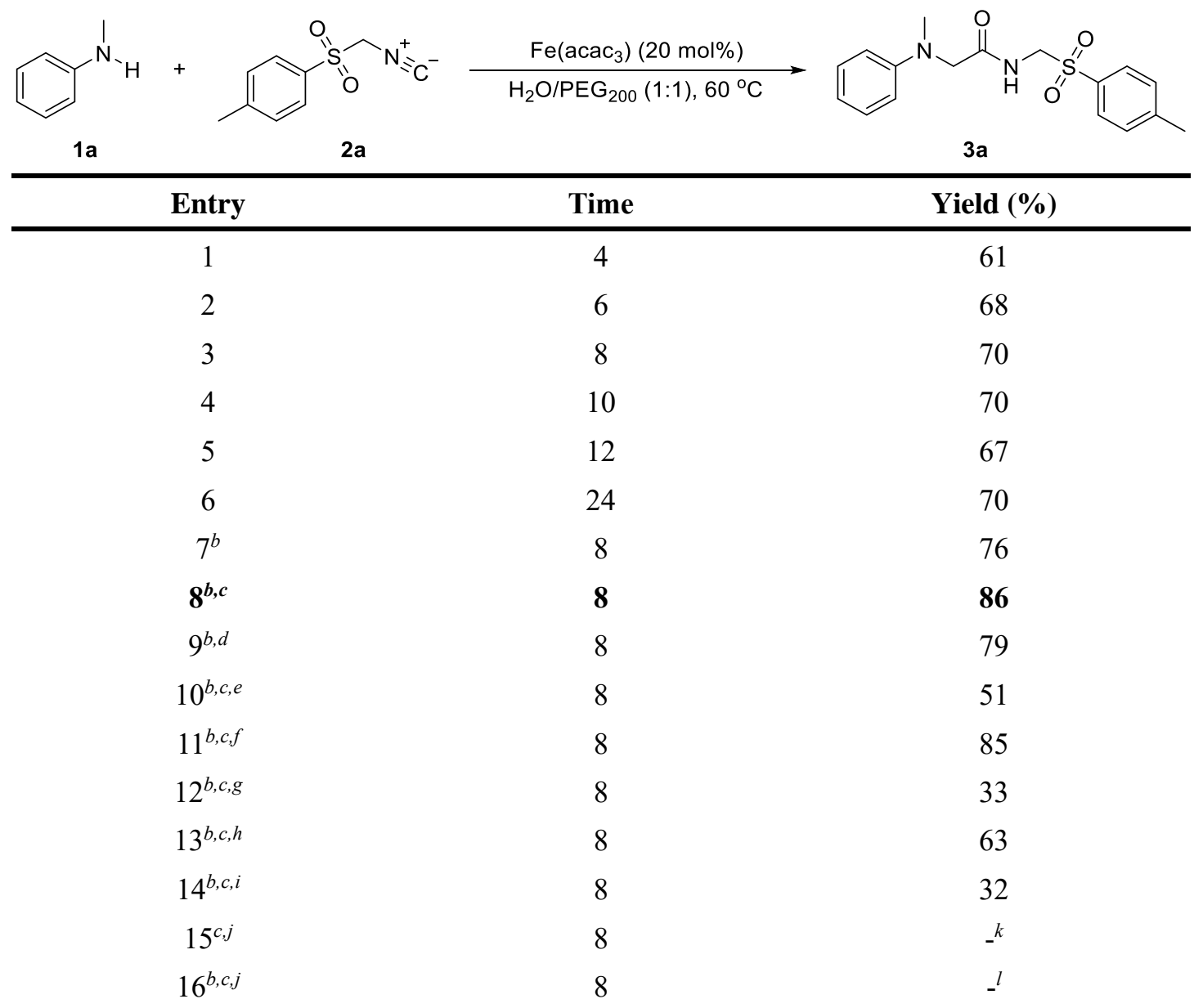

${ }^{a}$ Reaction conditions: 1a $(0.2 \mathrm{mmol}), \mathbf{2 a}(0.6 \mathrm{mmol}), \mathrm{Fe}(\mathrm{acac})_{3}\left(20 \mathrm{~mol}^{\circ}\right), \mathrm{H}_{2} \mathrm{O} / \mathrm{PEG}_{200}(1: 1)(2$ $\mathrm{mL}), 60{ }^{\circ} \mathrm{C}, 10 \mathrm{~h}$, under air. ${ }^{b}$ under Ar. ${ }^{c} \mathrm{H}_{2} \mathrm{O} / \mathrm{PEG}_{400}(1 / 1)(2 \mathrm{~mL}) .{ }^{d} \mathrm{H}_{2} \mathrm{O} / \mathrm{PEG}_{600}(1 / 1)(2 \mathrm{~mL}) .{ }^{e} \mathbf{2 a}$ $(0.4 \mathrm{mmol}) .{ }^{f_{2} \mathrm{a}}(0.8 \mathrm{mmol}) .{ }^{g}$ Without $\mathrm{Fe}(\mathrm{acac})_{3} .{ }^{h}$ Replacement of $\mathrm{Fe}(\mathrm{acac})_{3}$ with $\mathrm{Fe}(\mathrm{acac})_{2}$. ${ }^{i}$ Replacement of $\mathrm{Fe}(\mathrm{acac})_{3}$ with $\mathrm{K}_{3} \mathrm{Fe}(\mathrm{CN})_{6} .{ }^{j}$ Without $2 \mathrm{a} .{ }^{k} 29 \%$ of $1 \mathrm{a}$ was retrieved. ${ }^{1} 39 \%$ of $1 \mathrm{a}$ was retrieved. 


\section{General Procedure for the Preparation of 3, 5-10.}

General Procedure for 3, 5, and 6a-e: To a $15 \mathrm{~mL}$ sealed tube were added aniline derivatives (1.0 equiv, $0.2 \mathrm{mmol}$ ), TosMIC derivatives ( 3.0 equiv, $0.6 \mathrm{mmol})$, and $\mathrm{Fe}(\mathrm{acac})_{3}(0.2$ equiv, $0.04 \mathrm{mmol})$ in a mixed solvent of $\mathrm{PEG}_{400}$ and $\mathrm{H}_{2} \mathrm{O}(\mathrm{v} / \mathrm{v}=1 / 1,0.1 \mathrm{M}$ of aniline derivatives in $2 \mathrm{~mL}$ solvent $)$ under an $\mathrm{Ar}$ atmosphere. The reaction mixture was heated at $60{ }^{\circ} \mathrm{C}$ (oil bath) for several hours (8$24 \mathrm{~h})$. After cooling down to room temperature, the reaction mixture was diluted by $\mathrm{H}_{2} \mathrm{O}(10 \mathrm{~mL})$ and extracted by dichloromethane $(3 \times 10 \mathrm{~mL})$. The combined organic layers were dried by $\mathrm{Na}_{2} \mathrm{SO}_{4}$, filtered through Celite, and concentrated under reduced pressure. The residue was purified by preparative TLC on silica gel plates to give the corresponding products.

General Procedure for $\mathbf{6 f - i}$ : To a $15 \mathrm{~mL}$ sealed tube were added aniline derivatives (1.0 equiv, 0.2 $\mathrm{mmol}$ ), TosMIC 2a (1.0 equiv, $0.2 \mathrm{mmol}$ ), isocyanide derivatives 2' (3.0 equiv, $0.6 \mathrm{mmol}$ ), and $\mathrm{Fe}(\mathrm{acac})_{3}(0.2$ equiv, $0.04 \mathrm{mmol})$ in a mixed solvent of $\mathrm{PEG}_{400}$ and $\mathrm{H}_{2} \mathrm{O}(\mathrm{v} / \mathrm{v}=1 / 1,0.1 \mathrm{M}$ of aniline derivatives in $2 \mathrm{~mL}$ solvent) under an Ar atmosphere. The reaction mixture was heated at $60^{\circ} \mathrm{C}$ (oil bath) for $24 \mathrm{~h}$. After cooling down to room temperature, the reaction mixture was diluted by $\mathrm{H}_{2} \mathrm{O}$ $(10 \mathrm{~mL})$ and extracted by dichloromethane $(3 \times 10 \mathrm{~mL})$. The combined organic layers were dried by $\mathrm{Na}_{2} \mathrm{SO}_{4}$, filtered through Celite, and concentrated under reduced pressure. The residue was purified by preparative TLC on silica gel plates using petroleum/ethyl acetate as the eluent to give the corresponding products.

Gram-Scale Synthesis of 3a: To a $500 \mathrm{~mL}$ Schlenk bottle were added 1a (10 mmol, $1.07 \mathrm{~g}), 2 \mathrm{a}(30$ mmol, $5.86 \mathrm{~g})$, and $\mathrm{Fe}(\mathrm{acac})_{3}(2 \mathrm{mmol}, 0.71 \mathrm{~g})$ in a mixed solvent of $\mathrm{PEG}_{400}$ and $\mathrm{H}_{2} \mathrm{O}(\mathrm{v} / \mathrm{v}=1 / 1$, $0.1 \mathrm{M}$ of $1 \mathrm{a}$ in $100 \mathrm{~mL}$ solvent) under an Ar atmosphere. The reaction mixture was heated at $60{ }^{\circ} \mathrm{C}$ (oil bath) for $8 \mathrm{~h}$. After cooling down to room temperature, the reaction mixture was diluted by $\mathrm{H}_{2} \mathrm{O}$ $(100 \mathrm{~mL})$ and extracted by dichloromethane $(3 \times 100 \mathrm{~mL})$. The combined organic layers were dried by $\mathrm{Na}_{2} \mathrm{SO}_{4}$, filtered through Celite, and concentrated under reduced pressure. The residue was purified through a silica gel column using petroleum ether/ethyl acetate $=5: 1$ as the eluent to give pure product 3a in $76 \%$ yield $(2.52 \mathrm{~g})$.

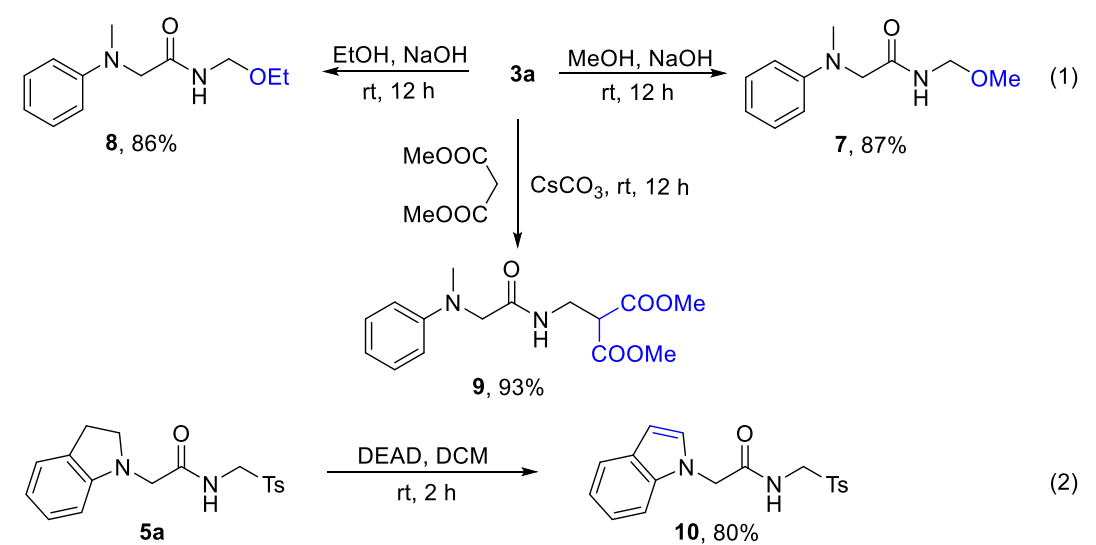

Scheme S1. Derivatization of $\alpha$-Amino Amides

Procedure for Preparation of 7: To a $15 \mathrm{~mL}$ sealed tube were added 3a $(0.2 \mathrm{mmol}, 66.5 \mathrm{mg})$ and $\mathrm{NaOH}(0.4 \mathrm{mmol}, 16.0 \mathrm{mg})$ in $\mathrm{CH}_{3} \mathrm{OH}\left(0.1 \mathrm{M}\right.$ of $3 \mathrm{a}$ in $\left.2 \mathrm{~mL} \mathrm{CH}_{3} \mathrm{OH}\right)$. The vessel was sealed with 
a Teflon-lined cap, and the reaction mixture was allowed to stir at the room temperature for $12 \mathrm{~h}$. After removal of solvent, the residue was purified by preparative TLC on silica gel plates using petroleum/ethyl acetate $=1 / 1$ as the eluent to afford 7 in $87 \%$ yield $(36.2 \mathrm{mg})$.

Procedure for Preparation of 8: To a $15 \mathrm{~mL}$ sealed tube were added 3a $(0.2 \mathrm{mmol}, 66.5 \mathrm{mg})$ and $\mathrm{NaOH}(0.4 \mathrm{mmol}, 16.0 \mathrm{mg})$ in EtOH $(0.1 \mathrm{M}$ of 3a in $2 \mathrm{~mL} \mathrm{EtOH})$. The vessel was sealed with a Teflon-lined cap, and the reaction mixture was allowed to stir at the room temperature for $12 \mathrm{~h}$. After removal of solvent, the residue was purified by preparative TLC on silica gel plates using petroleum/ethyl acetate $=1 / 1$ as the eluent to afford $\mathbf{8}$ in $86 \%$ yield $(38.2 \mathrm{mg})$.

Procedure for Preparation of 9: To a $15 \mathrm{~mL}$ sealed tube were added 3a $(0.2 \mathrm{mmol}, 66.5 \mathrm{mg})$, Dimethyl malonate $(0.4 \mathrm{mmol}, 64.0 \mathrm{mg})$, and $\mathrm{Cs}_{2} \mathrm{CO}_{3}(0.4 \mathrm{mmol}, 130.3 \mathrm{mg})$ in dichloromethane ( $0.1 \mathrm{M}$ of $\mathbf{3 a}$ in $2 \mathrm{~mL}$ dichloromethane). The vessel was sealed with a Teflon-lined cap, and the reaction mixture was allowed to stir at the room temperature for $12 \mathrm{~h}$. After removal of solvent, the residue was purified by preparative TLC on silica gel plates using petroleum/ethyl acetate $=1 / 1$ as the eluent to afford 9 in $93 \%$ yield $(57.4 \mathrm{mg})$.

Procedure for Preparation of 10: To a solution of 3a $(0.2 \mathrm{mmol}, 66.5 \mathrm{mg})$ in dichloromethane $(0.02 \mathrm{M}$ of $3 \mathrm{a}$ in $10 \mathrm{~mL}$ dichloromethane), Diethyl azodicarboxylate $(0.22 \mathrm{mmol}, 38.3 \mathrm{mg})$ was added. The reaction mixture was allowed to stir at the room temperature for $2 \mathrm{~h}$. After removal of solvent, the residue was purified by preparative TLC on silica gel plates using dichloromethane/ethyl acetate $=10 / 1$ as the eluent to afford $\mathbf{1 0}$ in $80 \%$ yield $(54.8 \mathrm{mg})$. 


\section{Control Experiments}

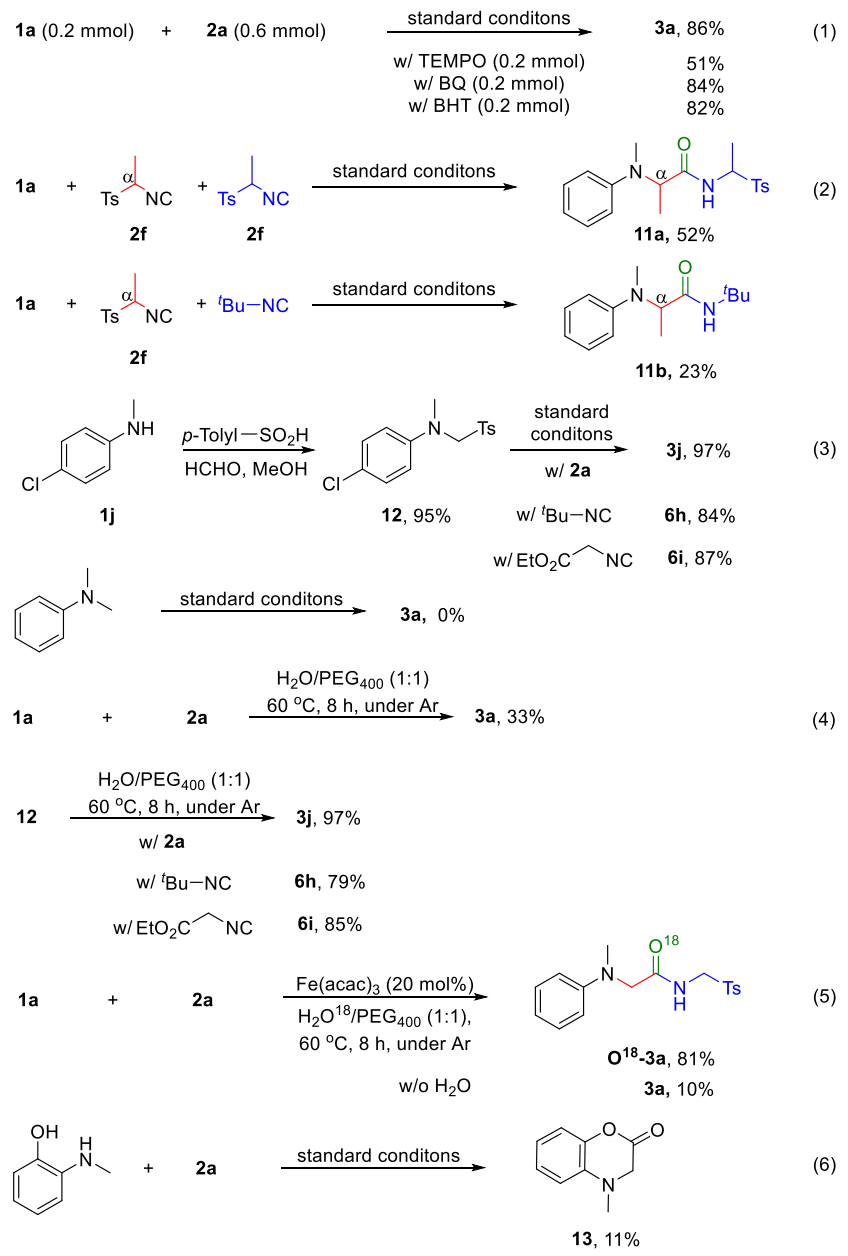

Scheme S2. Control Experiments

Reaction in the presence of radical scavengers. To a $15 \mathrm{~mL}$ sealed tube were added $1 \mathbf{a}(0.2 \mathrm{mmol}$, $21.4 \mathrm{mg}), 2 \mathrm{a}(0.6 \mathrm{mmol}, 117.1 \mathrm{mg}), \mathrm{Fe}(\mathrm{acac})_{3}(0.04 \mathrm{mmol}, 14.1 \mathrm{mg})$, and radical scavengers (TEMPO, BQ, or BHT, 1.0 equiv) in a mixed solvent of $\mathrm{PEG}_{400}$ and $\mathrm{H}_{2} \mathrm{O}(\mathrm{v} / \mathrm{v}=1 / 1,0.1 \mathrm{M}$ of $1 \mathrm{a}$ in $2 \mathrm{~mL}$ solvent) under an Ar atmosphere. The reaction mixture was heated at $60^{\circ} \mathrm{C}$ (oil bath) for $8 \mathrm{~h}$. After cooling down to room temperature, the reaction mixture was diluted by $\mathrm{H}_{2} \mathrm{O}(10 \mathrm{~mL})$ and extracted by dichloromethane $(3 \times 10 \mathrm{~mL})$. The combined organic layers were dried by $\mathrm{Na}_{2} \mathrm{SO}_{4}$, filtered through Celite, and concentrated under reduced pressure. The residue was purified by preparative TLC on silica gel plates using dichloromethane/ethyl acetate $=10: 1$ as the eluent to give the corresponding products $3 \mathbf{a}$ in 51,84 and $82 \%$ yields, respectively.

Procedure for Preparation of 11a: To a $15 \mathrm{~mL}$ sealed tube were added 1a $(0.2 \mathrm{mmol}, 21.4 \mathrm{mg}), 1$ methyl-1-tosylmethyl isocyanide $\mathbf{2 f}(0.6 \mathrm{mmol}, 125.5 \mathrm{mg})$, and Fe(acac) $3(0.04 \mathrm{mmol}, 14.1 \mathrm{mg})$ in a mixed solvent of $\mathrm{PEG}_{400}$ and $\mathrm{H}_{2} \mathrm{O}(\mathrm{v} / \mathrm{v}=1 / 1,0.1 \mathrm{M}$ of $1 \mathrm{a}$ in $2 \mathrm{~mL}$ solvent $)$ under an $\mathrm{Ar}$ atmosphere. The reaction mixture was heated at $60^{\circ} \mathrm{C}$ (oil bath) for $8 \mathrm{~h}$. After cooling down to room temperature, the reaction mixture was diluted by $\mathrm{H}_{2} \mathrm{O}(10 \mathrm{~mL})$ and extracted by dichloromethane $(3 \times 10 \mathrm{~mL})$. The combined organic layers were dried by $\mathrm{Na}_{2} \mathrm{SO}_{4}$, filtered through Celite, and concentrated under 
reduced pressure. The residue was purified by preparative TLC on silica gel plates using dichloromethane as the eluent to give the corresponding product $11 \mathrm{a}$ in $52 \%$ yield $(37.5 \mathrm{mg})$.

Procedure for Preparation of 11b: To a $15 \mathrm{~mL}$ sealed tube were added 1a $(0.2 \mathrm{mmol}, 21.4 \mathrm{mg})$, 1-methyl-1-tosylmethyl isocyanide $\mathbf{2 f}(0.2 \mathrm{mmol}, 41.8 \mathrm{mg})$, tert-butyl isocyanide (0.6 mmol, 49.9 $\mathrm{mg})$, and $\mathrm{Fe}(\mathrm{acac})_{3}(0.04 \mathrm{mmol}, 14.1 \mathrm{mg})$ in a mixed solvent of $\mathrm{PEG}_{400}$ and $\mathrm{H}_{2} \mathrm{O}(\mathrm{v} / \mathrm{v}=1 / 1,0.1 \mathrm{M}$ of $1 \mathrm{a}$ in $2 \mathrm{~mL}$ solvent) under an $\mathrm{Ar}$ atmosphere. The reaction mixture was heated at $60{ }^{\circ} \mathrm{C}$ (oil bath) for $8 \mathrm{~h}$. After cooling down to room temperature, the reaction mixture was diluted by $\mathrm{H}_{2} \mathrm{O}(10 \mathrm{~mL})$ and extracted by dichloromethane $(3 \times 10 \mathrm{~mL})$. The combined organic layers were dried by $\mathrm{Na}_{2} \mathrm{SO}_{4}$, filtered through Celite, and concentrated under reduced pressure. The residue was purified by preparative TLC on silica gel plates using dichloromethane as the eluent to give the corresponding product $11 \mathbf{b}$ in $23 \%$ yield $(10.8 \mathrm{mg})$.

Procedure for Preparation of 12: To a $50 \mathrm{~mL}$ round-bottom flask was added 1j (1.0 mmol, 141.6 $\mathrm{mg}$ ) and $p$-toluenesulfinic acid (1.1 mmol, $171.8 \mathrm{mg})$ in $\mathrm{MeOH}(0.1 \mathrm{M}$ of $\mathbf{1 j}$ in $10 \mathrm{~mL} \mathrm{MeOH})$. The mixture was cooled to $0{ }^{\circ} \mathrm{C}$, and aqueous formaldehyde ( $37 \%$ solution in water, $1.1 \mathrm{mmol}, 89.3 \mathrm{mg}$ ) was added. After 30 minutes, the solid was filtered and washed with $\mathrm{MeOH}$ without any other purification.

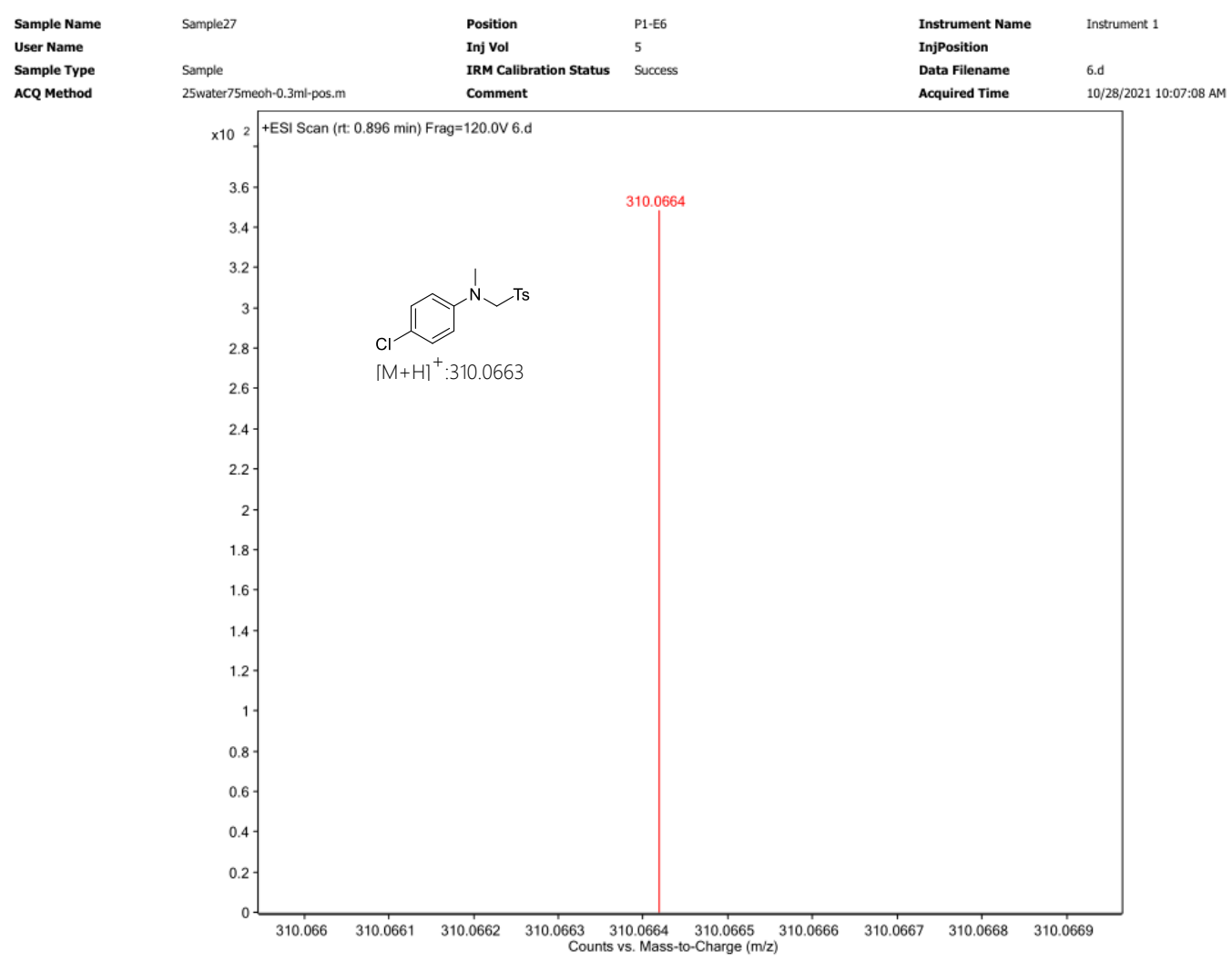

Figure S1. HRMS (positive ESI) for 12

Exploration of intermediates 12. To a $15 \mathrm{~mL}$ sealed tube were added $\mathbf{1 j}(0.2 \mathrm{mmol}, 28.3 \mathrm{mg}), \mathbf{2 a}$ $(0.6 \mathrm{mmol}, 117.1 \mathrm{mg})$, and $\mathrm{Fe}(\mathrm{acac})_{3}(0.04 \mathrm{mmol}, 14.1 \mathrm{mg})$ in a mixed solvent of $\mathrm{PEG}_{400}$ and $\mathrm{H}_{2} \mathrm{O}$ $(\mathrm{v} / \mathrm{v}=1 / 1,0.1 \mathrm{M}$ of $\mathbf{1 j}$ in $2 \mathrm{~mL}$ solvent) under an $\mathrm{Ar}$ atmosphere. The reaction mixture was heated at $60{ }^{\circ} \mathrm{C}$ (oil bath) for $1 \mathrm{~h}$. After cooling down to room temperature, the reaction mixture was 
concentrated directly. The HRMS spectra of intermediates $\mathbf{1 2}$ was determined as shown in Figure S2.

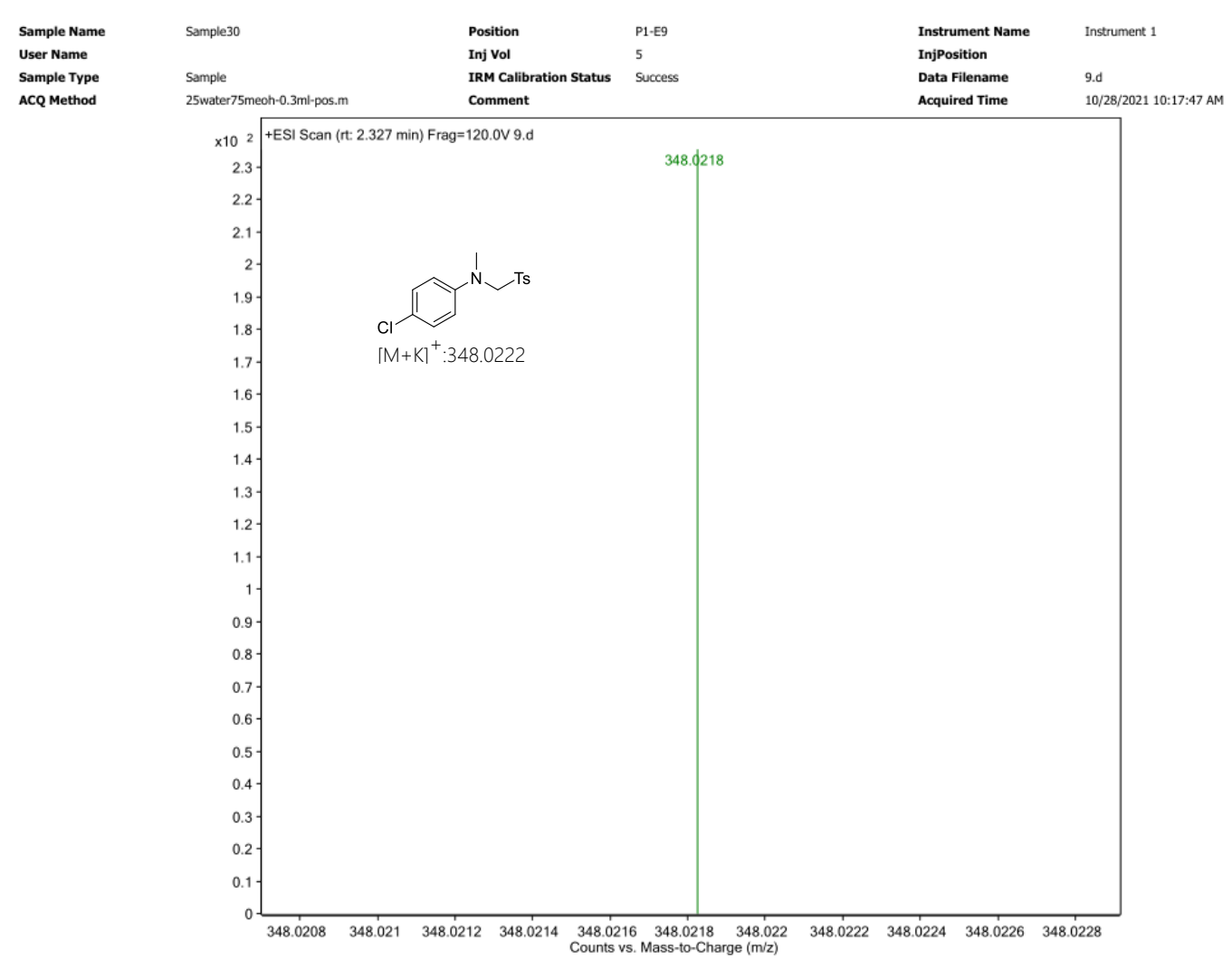

Figure S2. HRMS (positive ESI) for reaction residue

Reaction of 1a and 2a without $\mathrm{H}_{2} \mathrm{O}$. To a $15 \mathrm{~mL}$ sealed tube were added $\mathbf{1 a}(0.2 \mathrm{mmol}, 21.4 \mathrm{mg})$, 2a $(0.6 \mathrm{mmol}, 117.1 \mathrm{mg})$, and $\mathrm{Fe}(\mathrm{acac})_{3}(0.04 \mathrm{mmol}, 14.1 \mathrm{mg})$ in a solvent of $\mathrm{PEG}_{400}(\mathrm{v} / \mathrm{v}=1 / 1,0.1$ $\mathrm{M}$ of $1 \mathrm{a}$ in $2 \mathrm{~mL} \mathrm{PEG}_{400}$ ) under an $\mathrm{Ar}$ atmosphere. The reaction mixture was heated at $60{ }^{\circ} \mathrm{C}$ (oil bath) for $8 \mathrm{~h}$. After cooling down to room temperature, the reaction mixture was directly purified by preparative TLC on silica gel plates using dichloromethane/ethyl acetate $=10: 1$ as the eluent to afford $\mathbf{3 a}$ in $10 \%$ yield $(6.6 \mathrm{mg})$.

Reaction of 1a and 2a under $\mathbf{H}_{\mathbf{2}} \mathbf{O}^{\mathbf{1 8}}$. To a $15 \mathrm{~mL}$ sealed tube were added $\mathbf{1 a}(0.2 \mathrm{mmol}, 21.4 \mathrm{mg})$, 2a $(0.6 \mathrm{mmol}, 117.1 \mathrm{mg})$, and $\mathrm{Fe}(\mathrm{acac})_{3}(0.04 \mathrm{mmol}, 14.1 \mathrm{mg})$ in a mixed solvent of $\mathrm{PEG}_{400}$ and $\mathrm{H}_{2} \mathrm{O}^{18}(\mathrm{v} / \mathrm{v}=1 / 1,0.1 \mathrm{M}$ of $\mathbf{1 a}$ in $2 \mathrm{~mL}$ solvent) under an $\mathrm{Ar}$ atmosphere. The reaction mixture was heated at $60^{\circ} \mathrm{C}$ (oil bath) for $8 \mathrm{~h}$. After cooling down to room temperature, the reaction mixture was directly evaporated, and the residue was purified by preparative TLC on silica gel plates using dichloromethane/ethyl acetate $=10: 1$ as the eluent to afford ${ }^{18} \mathbf{O}-3 \mathbf{a}$ in $81 \%$ yield $(53.8 \mathrm{mg})$. 


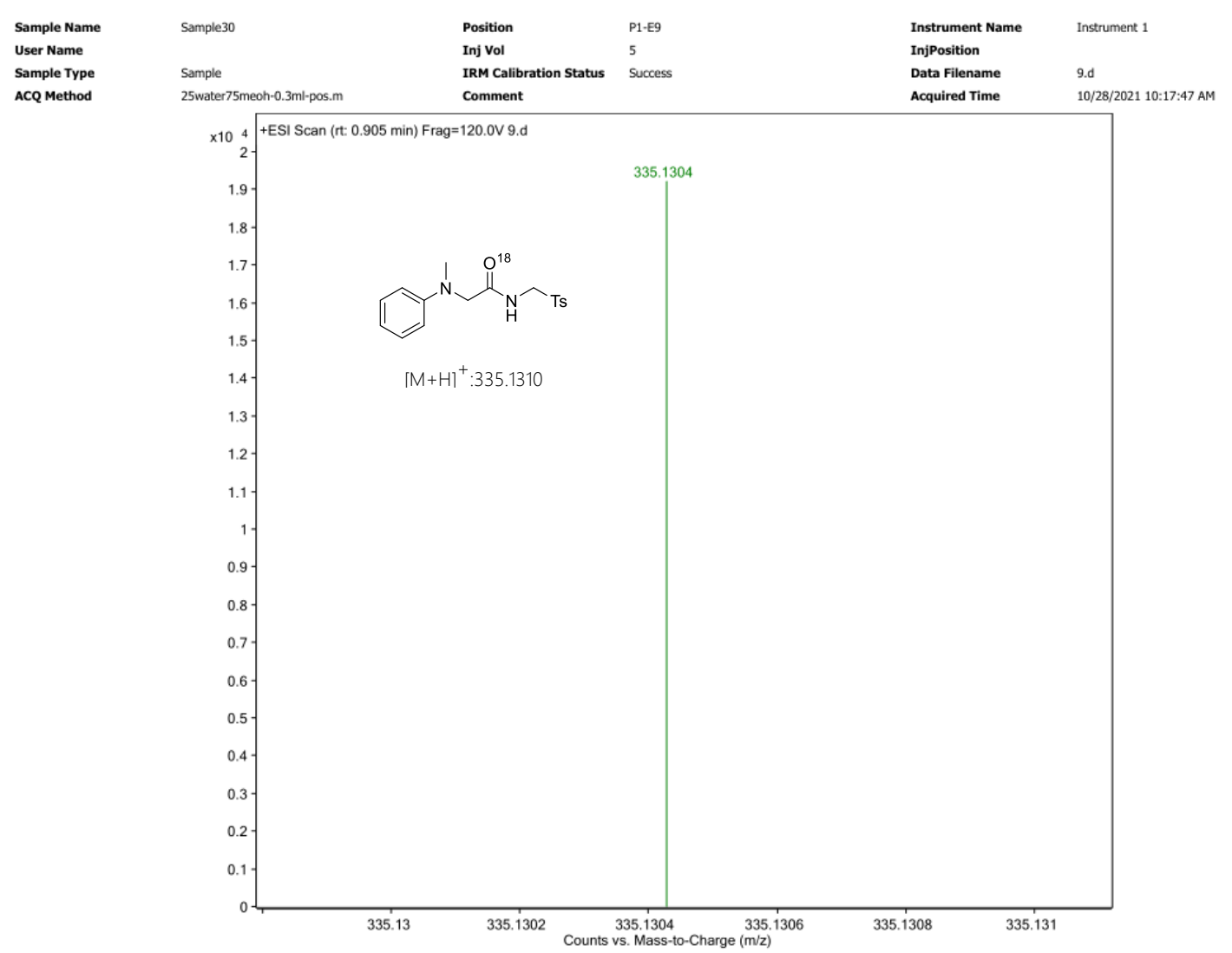

Figure S3. HRMS (positive ESI) for ${ }^{\mathbf{1 8}} \mathbf{O}-\mathbf{3 a}$

Procedure for Preparation of 13: To a $15 \mathrm{~mL}$ sealed tube were added 2-methylaminophenol $(0.2$ mmol, $24.6 \mathrm{mg}), 2 \mathrm{a}(0.6 \mathrm{mmol}, 117.1 \mathrm{mg})$, and $\mathrm{Fe}(\mathrm{acac})_{3}(0.04 \mathrm{mmol}, 14.1 \mathrm{mg})$ in a mixed solvent of $\mathrm{PEG}_{400}$ and $\mathrm{H}_{2} \mathrm{O}(\mathrm{v} / \mathrm{v}=1 / 1,0.1 \mathrm{M}$ of 2-methylaminophenol in $2 \mathrm{~mL}$ solvent) under an $\mathrm{Ar}$ atmosphere. The reaction mixture was heated at $60{ }^{\circ} \mathrm{C}$ (oil bath) for $8 \mathrm{~h}$. After cooling down to room temperature, the reaction mixture was diluted by $\mathrm{H}_{2} \mathrm{O}(10 \mathrm{~mL})$ and extracted by dichloromethane ( 3 $\times 10 \mathrm{~mL}$ ). The combined organic layers were dried by $\mathrm{Na}_{2} \mathrm{SO}_{4}$, filtered through Celite, and concentrated under reduced pressure. The residue was purified by preparative TLC on silica gel plates using petroleum/ethyl acetate $=5 / 1$ as the eluent to give the corresponding product $\mathbf{1 3}$ in $11 \%$ yield (3.6 mg). 


\section{Characterization for Products}

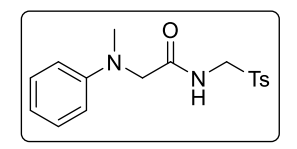

2-(Methyl(phenyl)amino)- $N$-(tosylmethyl)acetamide (3a). Purified by analytical TLC on silica gel with dichloromethane/ethyl acetate $=10: 1$ as an eluent with $\mathrm{R}_{\mathrm{f}}=0.4$, white solid (57.2 $\left.\mathrm{mg}, 86 \%\right)$. The spectroscopic data correspond to those previously reported in the literature. ${ }^{3} .{ }^{1} \mathrm{H}$ NMR $\left(600 \mathrm{MHz}, \mathrm{CDCl}_{3}\right) \delta 7.72(\mathrm{~d}, J$ $=7.8 \mathrm{~Hz}, 2 \mathrm{H}), 7.34-7.26(\mathrm{~m}, 5 \mathrm{H}), 6.89(\mathrm{t}, J=7.3 \mathrm{~Hz}, 1 \mathrm{H}), 6.67(\mathrm{~d}, J=8.1 \mathrm{~Hz}, 2 \mathrm{H}), 4.68(\mathrm{~d}, J=$ $6.8 \mathrm{~Hz}, 2 \mathrm{H}), 3.75(\mathrm{~s}, 2 \mathrm{H}), 2.98(\mathrm{~s}, 3 \mathrm{H}), 2.45(\mathrm{~s}, 3 \mathrm{H}) .{ }^{13} \mathrm{C}\left\{{ }^{1} \mathrm{H}\right\} \mathrm{NMR}\left(151 \mathrm{MHz}, \mathrm{CDCl}_{3}\right) \delta 170.3$, 149.0, 145.5, 133.8, 129.9, 129.5, 128.8, 119.2, 113.4, 59.9, 58.5, 40.0, 21.8. HRMS (ESI) $m / z$ : (M $+\mathrm{H})^{+}$calcd for $\mathrm{C}_{17} \mathrm{H}_{21} \mathrm{~N}_{2} \mathrm{O}_{3} \mathrm{~S}^{+}, 333.1268$; found, 333.1268 .

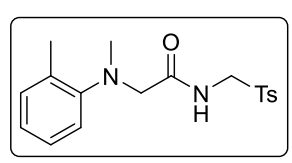

2-(Methyl(o-tolyl)amino)- $N$-(tosylmethyl)acetamide (3b). Purified by analytical TLC on silica gel with dichloromethane/ethyl acetate $=10: 1$ as an eluent with $\mathrm{R}_{\mathrm{f}}=0.4$, yellow oil $(48.5 \mathrm{mg}, 70 \%)$. The spectroscopic data correspond to those previously reported in the literature. ${ }^{4} \mathrm{H}$ NMR $(600 \mathrm{MHz}$, $\left.\mathrm{CDCl}_{3}\right) \delta 7.93(\mathrm{br}, 1 \mathrm{H}), 7.66(\mathrm{~d}, J=8.2 \mathrm{~Hz}, 2 \mathrm{H}), 7.26(\mathrm{~d}, J=7.0 \mathrm{~Hz}, 2 \mathrm{H}), 7.22(\mathrm{~d}, J=7.4 \mathrm{~Hz}, 1 \mathrm{H})$, $7.18(\mathrm{t}, J=7.6 \mathrm{~Hz}, 1 \mathrm{H}), 7.07-7.03(\mathrm{~m}, 2 \mathrm{H}), 4.74(\mathrm{~d}, J=6.9 \mathrm{~Hz}, 2 \mathrm{H}), 3.47(\mathrm{~s}, 2 \mathrm{H}), 2.65(\mathrm{~s}, 3 \mathrm{H})$, $2.41(\mathrm{~s}, 3 \mathrm{H}), 2.35$ (s, 3H). ${ }^{13} \mathrm{C}\left\{{ }^{1} \mathrm{H}\right\} \mathrm{NMR}\left(101 \mathrm{MHz}, \mathrm{CDCl}_{3}\right) \delta 169.9,150.6,145.4,134.0,132.5$, 131.6, 130.0, 128.7, 127.1, 124.7, 120.3, 60.2, 59.9, 43.6, 21.8, 18.5. HRMS (ESI) $m / z:(\mathrm{M}+\mathrm{H})^{+}$ calcd for $\mathrm{C}_{18} \mathrm{H}_{23} \mathrm{~N}_{2} \mathrm{O}_{3} \mathrm{~S}^{+}$, 347.1424; found, 347.1428.

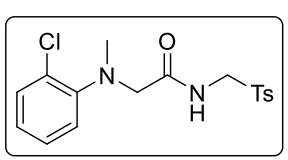

\section{2-((2-Chlorophenyl)(methyl)amino)- $N$-(tosylmethyl)acetamide}

(3c). Purified by analytical TLC on silica gel with dichloromethane/ethyl acetate $=$ 10:1 as an eluent with $\mathrm{R}_{\mathrm{f}}=0.4$, white solid ( $32.3 \mathrm{mg}, 44 \%$ ). The spectroscopic data correspond to those previously reported in the literature. ${ }^{4}{ }^{1} \mathrm{H}$ NMR (600 $\left.\mathrm{MHz}, \mathrm{CDCl}_{3}\right) \delta 8.08(\mathrm{t}, J=6.6 \mathrm{~Hz}, 1 \mathrm{H}), 7.75(\mathrm{~d}, J=7.8 \mathrm{~Hz}, 2 \mathrm{H}), 7.39(\mathrm{~d}, J=7.9 \mathrm{~Hz}, 1 \mathrm{H}), 7.31(\mathrm{~d}$, $J=7.9 \mathrm{~Hz}, 2 \mathrm{H}), 7.25$ (t, $J=7.7 \mathrm{~Hz}, 1 \mathrm{H}), 7.13(\mathrm{~d}, J=8.0 \mathrm{~Hz}, 1 \mathrm{H}), 7.06$ (t, $J=7.6 \mathrm{~Hz}, 1 \mathrm{H}), 4.77$ (d, $J=7.0 \mathrm{~Hz}, 2 \mathrm{H}), 3.52(\mathrm{~s}, 2 \mathrm{H}), 2.73(\mathrm{~s}, 3 \mathrm{H}), 2.43(\mathrm{~s}, 3 \mathrm{H}) .{ }^{13} \mathrm{C}\left\{{ }^{1} \mathrm{H}\right\} \mathrm{NMR}\left(101 \mathrm{MHz}, \mathrm{CDCl}_{3}\right) \delta 169.8$, 148.3, 145.4, 134.1, 130.7, 129.9, 129.1, 128.7, 127.9, 125.3, 121.9, 60.2, 60.0, 42.0, 21.8. HRMS (ESI) $m / z:(\mathrm{M}+\mathrm{H})^{+}$calcd for $\mathrm{C}_{17} \mathrm{H}_{20} \mathrm{ClN}_{2} \mathrm{O}_{3} \mathrm{~S}^{+}, 367.0878$; found, 367.0881 .

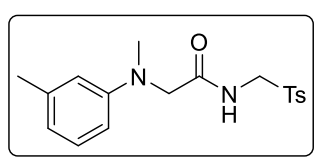

2-(Methyl( $m$-tolyl)amino)- $N$-(tosylmethyl)acetamide (3d). Purified by analytical TLC on silica gel with dichloromethane/ethyl acetate $=10: 1$ as an eluent with $\mathrm{R}_{\mathrm{f}}=0.4$, white solid (51.3 mg, 74\%). The spectroscopic data correspond to those previously reported in the literature. ${ }^{4}{ }^{1} \mathrm{H}$ NMR $(600$ $\left.\mathrm{MHz}, \mathrm{CDCl}_{3}\right) \delta 7.71(\mathrm{~d}, J=8.2 \mathrm{~Hz}, 2 \mathrm{H}), 7.36(\mathrm{t}, J=6.6 \mathrm{~Hz}, 1 \mathrm{H}), 7.32(\mathrm{~d}, J=8.0 \mathrm{~Hz}, 2 \mathrm{H}), 7.16(\mathrm{t}$, $J=7.8 \mathrm{~Hz}, 1 \mathrm{H}), 6.70(\mathrm{~d}, J=7.4 \mathrm{~Hz}, 1 \mathrm{H}), 6.52(\mathrm{~s}, 1 \mathrm{H}), 6.47(\mathrm{dd}, J=8.2,2.5 \mathrm{~Hz}, 1 \mathrm{H}), 4.66(\mathrm{~d}, J=$ $6.9 \mathrm{~Hz}, 2 \mathrm{H}), 3.74(\mathrm{~s}, 2 \mathrm{H}), 2.96(\mathrm{~s}, 3 \mathrm{H}), 2.45(\mathrm{~s}, 3 \mathrm{H}), 2.33(\mathrm{~s}, 3 \mathrm{H}) .{ }^{13} \mathrm{C}\left\{{ }^{1} \mathrm{H}\right\} \mathrm{NMR}\left(151 \mathrm{MHz}, \mathrm{CDCl}_{3}\right)$ $\delta 170.4,149.1,145.5,139.4,133.9,129.9,129.3,128.8,120.2,114.3,110.7,59.9,58.6,40.1,21.8$, 21.7. HRMS (ESI) $m / z$ : $(\mathrm{M}+\mathrm{H})^{+}$calcd for $\mathrm{C}_{18} \mathrm{H}_{23} \mathrm{~N}_{2} \mathrm{O}_{3} \mathrm{~S}^{+}$, 347.1424; found, 347.1429. 


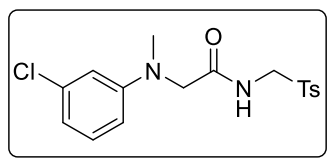

2-((3-Chlorophenyl)(methyl)amino)- $N$-(tosylmethyl)acetamide (3e).

Purified by analytical TLC on silica gel with dichloromethane/ethyl acetate $=10: 1$ as an eluent with $\mathrm{R}_{\mathrm{f}}=0.3$, white solid (46.2 $\mathrm{mg}, 63 \%$ ). The spectroscopic data correspond to those previously reported in the literature. ${ }^{4}{ }^{1} \mathrm{H}$ NMR (600 MHz, $\left.\mathrm{CDCl}_{3}\right) \delta 7.72(\mathrm{~d}, J=8.2 \mathrm{~Hz}, 2 \mathrm{H}), 7.34(\mathrm{~d}, J=8.2 \mathrm{~Hz}, 2 \mathrm{H}), 7.25(\mathrm{t}$, $J=6.2 \mathrm{~Hz}, 1 \mathrm{H}), 7.17(\mathrm{t}, J=8.1 \mathrm{~Hz}, 1 \mathrm{H}), 6.83(\mathrm{dt}, J=7.9,0.8 \mathrm{~Hz}, 1 \mathrm{H}), 6.64(\mathrm{t}, J=2.1 \mathrm{~Hz}, 1 \mathrm{H}), 6.52$ $(\mathrm{dd}, J=8.4,2.5 \mathrm{~Hz}, 1 \mathrm{H}), 4.67(\mathrm{~d}, J=6.8 \mathrm{~Hz}, 2 \mathrm{H}), 3.75(\mathrm{~s}, 2 \mathrm{H}), 2.97(\mathrm{~s}, 3 \mathrm{H}), 2.45(\mathrm{~s}, 3 \mathrm{H}) .{ }^{13} \mathrm{C}\left\{{ }^{1} \mathrm{H}\right\}$ NMR $\left(151 \mathrm{MHz}, \mathrm{CDCl}_{3}\right) \delta 169.7,150.0,145.7,135.4,133.7,130.4,130.0,128.8,119.0,113.3$, 111.4, 59.9, 58.0, 39.9, 21.8. HRMS (ESI) $m / z$ : $(\mathrm{M}+\mathrm{H})^{+}$calcd for $\mathrm{C}_{17} \mathrm{H}_{20} \mathrm{ClN}_{2} \mathrm{O}_{3} \mathrm{~S}^{+}, 367.0878$; found, 367.0877 .

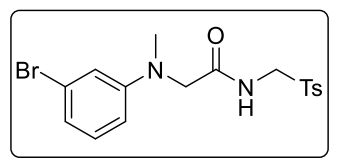

2-((3-Bromophenyl)(methyl)amino)- $N$-(tosylmethyl)acetamide

(3f). Purified by analytical TLC on silica gel with dichloromethane/ethyl acetate $=10: 1$ as an eluent with $\mathrm{R}_{\mathrm{f}}=0.3$, white solid (58.4 $\mathrm{mg}, 71 \%$ ). The spectroscopic data correspond to those previously reported in the literature. ${ }^{5}{ }^{1} \mathrm{H}$ NMR $\left(600 \mathrm{MHz}, \mathrm{CDCl}_{3}\right) \delta 7.71(\mathrm{~d}, J=8.2 \mathrm{~Hz}, 2 \mathrm{H}), 7.35(\mathrm{~d}, J=8.1 \mathrm{~Hz}, 2 \mathrm{H}), 7.25(\mathrm{t}$, $J=6.6 \mathrm{~Hz}, 1 \mathrm{H}), 7.11(\mathrm{t}, J=8.1 \mathrm{~Hz}, 1 \mathrm{H}), 6.98(\mathrm{~d}, J=7.9 \mathrm{~Hz}, 1 \mathrm{H}), 6.81(\mathrm{t}, J=1.9 \mathrm{~Hz}, 1 \mathrm{H}), 6.56(\mathrm{dd}$, $J=8.3,2.4 \mathrm{~Hz}, 1 \mathrm{H}), 4.67(\mathrm{~d}, J=6.9 \mathrm{~Hz}, 2 \mathrm{H}), 3.75(\mathrm{~s}, 2 \mathrm{H}), 2.96(\mathrm{~s}, 3 \mathrm{H}), 2.45(\mathrm{~s}, 3 \mathrm{H}) .{ }^{13} \mathrm{C}\left\{{ }^{1} \mathrm{H}\right\} \mathrm{NMR}$ $\left(101 \mathrm{MHz} \mathrm{CDCl}_{3}\right) \delta 169.7,150.2,145.7,133.7,130.7,130.1,128.8,123.6,121.9,116.1,111.8$, 59.9, 57.9, 39.9, 21.8. HRMS (ESI) $m / z$ : $(\mathrm{M}+\mathrm{H})^{+}$calcd for $\mathrm{C}_{17} \mathrm{H}_{20} \mathrm{BrN}_{2} \mathrm{O}_{3} \mathrm{~S}^{+}, 411.0373$; found, 411.0376 .

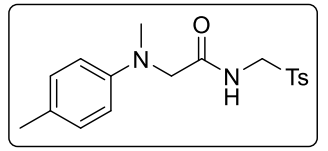

2-(Methyl(p-tolyl)amino)-N-(tosylmethyl)acetamide (3g). Purified by analytical TLC on silica gel with dichloromethane/ethyl acetate $=10: 1$ as an eluent with $\mathrm{R}_{\mathrm{f}}=0.4$, white solid (43.6 mg, 63\%). The spectroscopic data correspond to those previously reported in the literature. ${ }^{5}{ }^{1} \mathrm{H}$ NMR $(600$ $\left.\mathrm{MHz}, \mathrm{CDCl}_{3}\right) \delta 7.72(\mathrm{~d}, J=8.1 \mathrm{~Hz}, 2 \mathrm{H}), 7.42(\mathrm{br}, 1 \mathrm{H}), 7.33(\mathrm{~d}, J=8.1 \mathrm{~Hz}, 2 \mathrm{H}), 7.08(\mathrm{~d}, J=8.3 \mathrm{~Hz}$, 2H), 6.59 (d, $J=8.4 \mathrm{~Hz}, 2 \mathrm{H}), 4.67$ (d, $J=6.9 \mathrm{~Hz}, 2 \mathrm{H}), 3.69(\mathrm{~s}, 2 \mathrm{H}), 2.94(\mathrm{~s}, 3 \mathrm{H}), 2.45$ (s, 3H), 2.29 (s, 3H). ${ }^{13} \mathrm{C}\left\{{ }^{1} \mathrm{H}\right\} \mathrm{NMR}\left(151 \mathrm{MHz}, \mathrm{CDCl}_{3}\right) \delta 170.5,147.0,145.5,133.8,129.9,129.9,128.8,128.7$, 113.8, 59.9, 58.9, 40.3, 21.8, 20.3. HRMS (ESI) $m / z:(\mathrm{M}+\mathrm{H})^{+}$calcd for $\mathrm{C}_{18} \mathrm{H}_{23} \mathrm{~N}_{2} \mathrm{O}_{3} \mathrm{~S}^{+}, 347.1424$; found, 347.1426 .

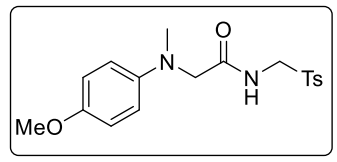

2-((4-Methoxyphenyl)(methyl)amino)- $N$-(tosylmethyl)acetamide (3h). Purified by analytical TLC on silica gel with dichloromethane/ethyl acetate $=10: 1$ as an eluent with $\mathrm{R}_{\mathrm{f}}=0.3$, white solid $(50.0 \mathrm{mg}, 69 \%)$. The spectroscopic data correspond to those previously reported in the literature. ${ }^{3}{ }^{1} \mathrm{H}$ NMR $\left(400 \mathrm{MHz}, \mathrm{CDCl}_{3}\right) \delta 7.72(\mathrm{~d}, J=8.3 \mathrm{~Hz}, 2 \mathrm{H}), 7.57(\mathrm{t}, J=6.8 \mathrm{~Hz}, 1 \mathrm{H}), 7.33(\mathrm{~d}$, $J=8.0 \mathrm{~Hz}, 2 \mathrm{H}), 6.87-6.83(\mathrm{~m}, 2 \mathrm{H}), 6.68-6.64(\mathrm{~m}, 2 \mathrm{H}), 4.69(\mathrm{~d}, J=6.9 \mathrm{~Hz}, 2 \mathrm{H}), 3.77(\mathrm{~s}, 3 \mathrm{H})$, $3.64(\mathrm{~s}, 2 \mathrm{H}), 2.90(\mathrm{~s}, 3 \mathrm{H}), 2.45(\mathrm{~s}, 3 \mathrm{H}) .{ }^{13} \mathrm{C}\left\{{ }^{1} \mathrm{H}\right\} \mathrm{NMR}\left(101 \mathrm{MHz}, \mathrm{CDCl}_{3}\right) \delta 170.6,153.5,145.5$, 143.7, 133.8, 129.9, 128.8, 115.6, 114.8, 59.9, 59.5, 55.7, 41.0, 21.8. HRMS (ESI) $m / z:(\mathrm{M}+\mathrm{H})^{+}$ calcd for $\mathrm{C}_{18} \mathrm{H}_{23} \mathrm{~N}_{2} \mathrm{O}_{4} \mathrm{~S}^{+}, 363.1373$; found, 363.1373 . 


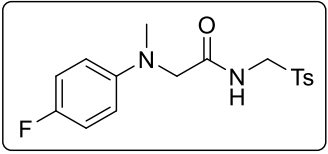

2-((4-Fluorophenyl)(methyl)amino)- $N$-(tosylmethyl)acetamide

(3i).

Purified by analytical TLC on silica gel with dichloromethane/ethyl acetate $=10: 1$ as an eluent with $\mathrm{R}_{\mathrm{f}}=0.4$, white solid $(55.4 \mathrm{mg}, 79 \%)$. The spectroscopic data correspond to those previously reported in the literature. ${ }^{3}{ }^{1} \mathrm{H}$ NMR $\left(600 \mathrm{MHz}, \mathrm{CDCl}_{3}\right) \delta 7.71(\mathrm{~d}, J=8.2 \mathrm{~Hz}, 2 \mathrm{H}), 7.43(\mathrm{t}, J=6.4 \mathrm{~Hz}, 1 \mathrm{H}), 7.34(\mathrm{~d}$, $J=8.0 \mathrm{~Hz}, 2 \mathrm{H}), 6.97(\mathrm{t}, J=8.6 \mathrm{~Hz}, 2 \mathrm{H}), 6.63-6.60(\mathrm{~m}, 2 \mathrm{H}), 4.69$ (d, $J=6.8 \mathrm{~Hz}, 2 \mathrm{H}), 3.70(\mathrm{~s}, 2 \mathrm{H})$, $2.94(\mathrm{~s}, 3 \mathrm{H}), 2.46(\mathrm{~s}, 3 \mathrm{H}) \cdot{ }^{13} \mathrm{C}\left\{{ }^{1} \mathrm{H}\right\} \mathrm{NMR}\left(101 \mathrm{MHz}, \mathrm{CDCl}_{3}\right) \delta 170.2,156.8(\mathrm{~d}, J=239.4 \mathrm{~Hz}), 145.7$ (d, $J=2.1 \mathrm{~Hz}), 145.6,133.7,130.0,128.8,115.9$ (d, $J=22.3 \mathrm{~Hz}), 115.0$ (d, $J=7.7 \mathrm{~Hz}), 59.9,59.1$, 40.7, 21.8. ${ }^{19} \mathrm{~F}$ NMR $\left(565 \mathrm{MHz}, \mathrm{CDCl}_{3}\right) \delta$-125.47. HRMS (ESI) $\mathrm{m} / z:(\mathrm{M}+\mathrm{H})^{+}$calcd for $\mathrm{C}_{17} \mathrm{H}_{20} \mathrm{FN}_{2} \mathrm{O}_{3} \mathrm{~S}^{+}, 351.1173$; found, 351.1174 .

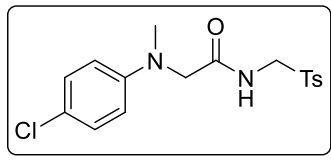

2-((4-Chlorophenyl)(methyl)amino)- $N$-(tosylmethyl)acetamide $\quad(3 \mathrm{j})$. Purified by analytical TLC on silica gel with dichloromethane/ethyl acetate $=10: 1$ as an eluent with $R_{\mathrm{f}}=0.3$, white solid $(55.0 \mathrm{mg}, 75 \%)$. The spectroscopic data correspond to those previously reported in the literature. ${ }^{3}{ }^{1} \mathrm{H}$ NMR $\left(600 \mathrm{MHz}, \mathrm{CDCl}_{3}\right) \delta 7.71(\mathrm{~d}, J=8.1 \mathrm{~Hz}, 2 \mathrm{H}), 7.36-7.32(\mathrm{~m}, 3 \mathrm{H}), 7.22(\mathrm{~d}, J$ $=8.9 \mathrm{~Hz}, 2 \mathrm{H}), 6.58(\mathrm{~d}, J=8.9 \mathrm{~Hz}, 2 \mathrm{H}), 4.69(\mathrm{~d}, J=6.8 \mathrm{~Hz}, 2 \mathrm{H}), 3.75(\mathrm{~s}, 2 \mathrm{H}), 2.99(\mathrm{~s}, 3 \mathrm{H}), 2.48(\mathrm{~s}$, $3 \mathrm{H}) .{ }^{13} \mathrm{C}\left\{{ }^{1} \mathrm{H}\right\}$ NMR $\left(151 \mathrm{MHz}, \mathrm{CDCl}_{3}\right) \delta 170.0,147.6,145.6,133.7,130.0,129.2,128.7,124.2$, 114.6, 59.9, 58.3, 40.2, 21.8. HRMS (ESI) $m / z$ : $(\mathrm{M}+\mathrm{H})^{+}$calcd for $\mathrm{C}_{17} \mathrm{H}_{20} \mathrm{ClN}_{2} \mathrm{O}_{3} \mathrm{~S}^{+}, 367.0878$; found, 367.0881 .

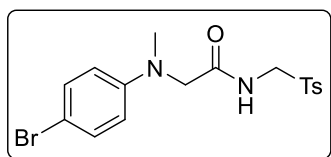

2-((4-Bromophenyl)(methyl)amino)- $N$-(tosylmethyl)acetamide $\quad(3 k)$. Purified by analytical TLC on silica gel with dichloromethane/ethyl acetate $=10: 1$ as an eluent with $R_{f}=0.2$, white solid $(62.5 \mathrm{mg}, 76 \%)$. The spectroscopic data correspond to those previously reported in the literature. ${ }^{3}{ }^{1} \mathrm{H}$ NMR $\left(600 \mathrm{MHz}, \mathrm{CDCl}_{3}\right) \delta 7.69(\mathrm{~d}, J=8.2 \mathrm{~Hz}, 2 \mathrm{H}), 7.33(\mathrm{~d}, J=8.6 \mathrm{~Hz}, 4 \mathrm{H}), 7.28(\mathrm{t}$, $J=6.3 \mathrm{~Hz}, 1 \mathrm{H}), 6.51(\mathrm{~d}, J=8.9 \mathrm{~Hz}, 2 \mathrm{H}), 4.68(\mathrm{~d}, J=6.8 \mathrm{~Hz}, 2 \mathrm{H}), 3.73(\mathrm{~s}, 2 \mathrm{H}), 2.97(\mathrm{~s}, 3 \mathrm{H}), 2.46$ (s, 3H). ${ }^{13} \mathrm{C}\left\{{ }^{1} \mathrm{H}\right\}$ NMR $\left(151 \mathrm{MHz}, \mathrm{CDCl}_{3}\right) \delta 169.9,148.0,145.6,133.7$ 132.1, 130.0, 128.7, 115.0, 111.3, 59.9, 58.2, 40.1, 21.8. HRMS (ESI) $m / z:(\mathrm{M}+\mathrm{H})^{+}$calcd for $\mathrm{C}_{17} \mathrm{H}_{20} \mathrm{BrN}_{2} \mathrm{O}_{3} \mathrm{~S}^{+}, 411.0373$; found, 411.0369 .

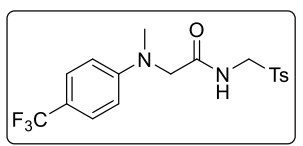

2-(Methyl(4-(trifluoromethyl)phenyl)amino)- $N$-(tosylmethyl)acetamide (3I). Purified by analytical TLC on silica gel with dichloromethane/ethyl acetate $=10: 1$ as an eluent with $\mathrm{R}_{\mathrm{f}}=0.2$, white solid $(50.5 \mathrm{mg}, 63 \%), \mathrm{mp}=$ 177-178 ${ }^{\circ} \mathrm{C} .{ }^{1} \mathrm{H}$ NMR $(600 \mathrm{MHz}, \mathrm{DMSO}) \delta 9.02(\mathrm{t}, J=6.6 \mathrm{~Hz}, 1 \mathrm{H}), 7.69(\mathrm{~d}, J=8.2 \mathrm{~Hz}, 2 \mathrm{H}), 7.43$ $(\mathrm{d}, J=8.8 \mathrm{~Hz}, 2 \mathrm{H}), 7.40(\mathrm{~d}, J=8.0 \mathrm{~Hz}, 2 \mathrm{H}), 6.60(\mathrm{~d}, J=8.8 \mathrm{~Hz}, 2 \mathrm{H}), 4.68(\mathrm{~d}, J=6.6 \mathrm{~Hz}, 2 \mathrm{H}), 4.00$ (s, 2H), $2.90(\mathrm{~s}, 3 \mathrm{H}), 2.37(\mathrm{~s}, 3 \mathrm{H}) .{ }^{13} \mathrm{C}\left\{{ }^{1} \mathrm{H}\right\}$ NMR (151 MHz, DMSO) $\delta 169.5,152.0,144.9,134.9$, 130.2, 129.0, 126.4 (q, $J=4.4 \mathrm{~Hz}), 125.7$ (q, $J=269.7 \mathrm{~Hz}), 116.3$ (q, $J=31.9 \mathrm{~Hz}), 111.8,60.5,54.6$, 39.6, 21.5. ${ }^{19} \mathrm{~F}$ NMR $\left(565 \mathrm{MHz}\right.$, DMSO) $\delta$-59.03. HRMS (ESI) $m / z:(\mathrm{M}+\mathrm{H})^{+}$calcd for $\mathrm{C}_{18} \mathrm{H}_{20} \mathrm{~F}_{3} \mathrm{~N}_{2} \mathrm{O}_{3} \mathrm{~S}^{+}$, 401.1141; found, 401.1145 .

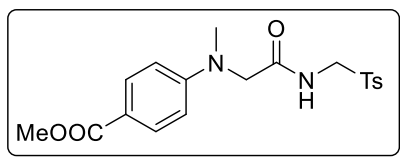

Methyl 4-(methyl(2-oxo-2-((tosylmethyl)amino)ethyl)amino) benzoate (3m). Purified by analytical TLC on silica gel with dichloromethane/ethyl acetate $=10: 1$ as an eluent with $\mathrm{R}_{\mathrm{f}}=0.2$, white solid (31.2 mg, 40\%), mp = 161-162 ${ }^{\circ} \mathrm{C} .{ }^{1} \mathrm{H}$ NMR $\left(600 \mathrm{MHz}, \mathrm{CDCl}_{3}\right)$ 
$\delta 7.91(\mathrm{~d}, J=9.0 \mathrm{~Hz}, 2 \mathrm{H}), 7.69(\mathrm{~d}, J=8.2 \mathrm{~Hz}, 2 \mathrm{H}), 7.33(\mathrm{~d}, J=8.0 \mathrm{~Hz}, 2 \mathrm{H}), 7.16(\mathrm{t}, J=6.9 \mathrm{~Hz}$, $1 \mathrm{H}), 6.58(\mathrm{~d}, J=9.1 \mathrm{~Hz}, 2 \mathrm{H}), 4.67$ (d, $J=6.8 \mathrm{~Hz}, 2 \mathrm{H}), 3.88(\mathrm{~s}, 3 \mathrm{H}), 3.86(\mathrm{~s}, 2 \mathrm{H}), 3.06(\mathrm{~s}, 3 \mathrm{H}), 2.45$ (s, 3H). ${ }^{13} \mathrm{C}\left\{{ }^{1} \mathrm{H}\right\} \mathrm{NMR}\left(101 \mathrm{MHz}, \mathrm{CDCl}_{3}\right) \delta 169.3,167.0,152.0,145.7,133.6,131.4,130.0,128.8$, 120.0, 111.8, 60.0, 57.3, 51.8, 39.8, 21.8. HRMS (ESI) $m / z:(\mathrm{M}+\mathrm{H})^{+}$calcd for $\mathrm{C}_{19} \mathrm{H}_{23} \mathrm{~N}_{2} \mathrm{O}_{5} \mathrm{~S}^{+}$, 391.1322; found, 391.1326 .

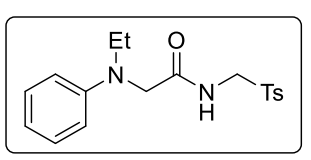

2-(Ethyl(phenyl)amino)- $N$-(tosylmethyl)acetamide (3n). Purified by analytical TLC on silica gel with dichloromethane/ethyl acetate $=10: 1$ as an eluent with $\mathrm{R}_{\mathrm{f}}=0.4$, white solid (50.6 mg, 73\%). The spectroscopic data correspond to those previously reported in the literature. ${ }^{5}{ }^{1} \mathrm{H}$ NMR $(600$ $\left.\mathrm{MHz}, \mathrm{CDCl}_{3}\right) \delta 7.67(\mathrm{~d}, J=8.1 \mathrm{~Hz}, 2 \mathrm{H}), 7.31-7.30(\mathrm{~m}, 3 \mathrm{H}), 7.27(\mathrm{t}, J=7.6 \mathrm{~Hz}, 2 \mathrm{H}), 6.86(\mathrm{t}, J=$ $7.3 \mathrm{~Hz}, 1 \mathrm{H}), 6.66(\mathrm{~d}, J=8.3 \mathrm{~Hz}, 2 \mathrm{H}), 4.65(\mathrm{~d}, J=6.9 \mathrm{~Hz}, 2 \mathrm{H}), 3.73(\mathrm{~s}, 2 \mathrm{H}), 3.41(\mathrm{q}, J=7.1 \mathrm{~Hz}$, 2H), 2.45 (s, 3H), $1.17(\mathrm{t}, J=7.1 \mathrm{~Hz}, 3 \mathrm{H}) .{ }^{13} \mathrm{C}\left\{{ }^{1} \mathrm{H}\right\} \mathrm{NMR}\left(151 \mathrm{MHz}, \mathrm{CDCl}_{3}\right) \delta$ 170.4, 147.4, 145.4, 133.9, 129.9, 129.6, 128.8, 119.0, 113.7, 59.9, 55.4, 46.5, 21.7, 11.5. HRMS (ESI) $m / z:(\mathrm{M}+\mathrm{H})^{+}$ calcd for $\mathrm{C}_{18} \mathrm{H}_{23} \mathrm{~N}_{2} \mathrm{O}_{3} \mathrm{~S}^{+}$, 347.1424; found, 347.1418.

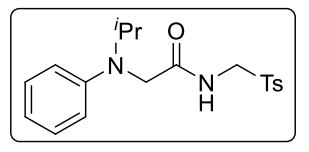

2-(Isopropyl(phenyl)amino)- $N$-(tosylmethyl)acetamide (30). Purified by analytical TLC on silica gel with dichloromethane/ethyl acetate $=10: 1$ as an eluent with $\mathrm{R}_{\mathrm{f}}=0.5$, white solid $(42.5 \mathrm{mg}, 59 \%), \mathrm{mp}=144-145^{\circ} \mathrm{C} .{ }^{1} \mathrm{H}$ NMR $\left(600 \mathrm{MHz}, \mathrm{CDCl}_{3}\right) \delta 7.65(\mathrm{~d}, J=8.2 \mathrm{~Hz}, 2 \mathrm{H}), 7.42(\mathrm{t}, J=6.4 \mathrm{~Hz}, 1 \mathrm{H}), 7.30-7.27(\mathrm{~m}, 4 \mathrm{H}), 6.89(\mathrm{t}$, $J=7.3 \mathrm{~Hz}, 1 \mathrm{H}), 6.73(\mathrm{~d}, J=8.3 \mathrm{~Hz}, 2 \mathrm{H}), 4.64(\mathrm{~d}, J=7.0 \mathrm{~Hz}, 2 \mathrm{H}), 4.17-4.10(\mathrm{~m}, 1 \mathrm{H}), 3.62(\mathrm{~s}, 2 \mathrm{H})$, $2.44(\mathrm{~s}, 3 \mathrm{H}), 1.16(\mathrm{~d}, J=6.7 \mathrm{~Hz}, 6 \mathrm{H}) .{ }^{13} \mathrm{C}\left\{{ }^{1} \mathrm{H}\right\}$ NMR $\left(101 \mathrm{MHz}, \mathrm{CDCl}_{3}\right) \delta 171.0,148.2,145.4$, $133.9,129.9,129.5,128.8,119.6,115.0,59.8,49.2,21.8,19.4$. HRMS (ESI) $m / z:(\mathrm{M}+\mathrm{H})^{+}$calcd for $\mathrm{C}_{19} \mathrm{H}_{25} \mathrm{~N}_{2} \mathrm{O}_{3} \mathrm{~S}^{+}, 361.1581$; found, 361.1584 .

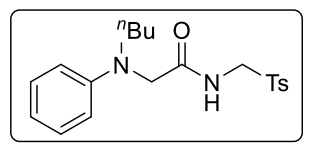

2-(Butyl(phenyl)amino)- $N$-(tosylmethyl)acetamide (3p). Purified by analytical TLC on silica gel with dichloromethane/ethyl acetate $=10: 1$ as an eluent with $\mathrm{R}_{\mathrm{f}}=0.3$, white solid (56.2 mg, 75\%), $\mathrm{mp}=112-113^{\circ} \mathrm{C} .{ }^{1} \mathrm{H}$ NMR $\left(600 \mathrm{MHz}, \mathrm{CDCl}_{3}\right) \delta 7.66(\mathrm{~d}, J=8.2 \mathrm{~Hz}, 2 \mathrm{H}), 7.30(\mathrm{~d}, J=8.0 \mathrm{~Hz}, 2 \mathrm{H}), 7.28-7.25(\mathrm{~m}, 2 \mathrm{H}), 7.22$ (br, 1H), 6.85 (t, $J=7.3 \mathrm{~Hz}, 1 \mathrm{H}), 6.64(\mathrm{~d}, J=8.2 \mathrm{~Hz}, 2 \mathrm{H}), 4.64$ (d, $J=6.9 \mathrm{~Hz}, 2 \mathrm{H}), 3.75(\mathrm{~s}, 2 \mathrm{H})$, $3.31(\mathrm{t}, J=7.9 \mathrm{~Hz}, 2 \mathrm{H}), 2.45$ (s, 3H), $1.59-1.54(\mathrm{~m}, 2 \mathrm{H}), 1.39-1.33(\mathrm{~m}, 2 \mathrm{H}), 0.97$ (t, $J=7.4 \mathrm{~Hz}$, $3 \mathrm{H}) .{ }^{13} \mathrm{C}\left\{{ }^{1} \mathrm{H}\right\}$ NMR $\left(101 \mathrm{MHz}, \mathrm{CDCl}_{3}\right) \delta 170.4,147.4,145.5,133.8,129.9,129.6,128.8,118.8$, 113.5, 59.8, 55.9, 52.3, 28.6, 21.8, 20.3, 13.9. HRMS (ESI) $\mathrm{m} / \mathrm{z}$ : $(\mathrm{M}+\mathrm{H})^{+}$calcd for $\mathrm{C}_{20} \mathrm{H}_{27} \mathrm{~N}_{2} \mathrm{O}_{3} \mathrm{~S}^{+}$, 375.1737; found, 375.1739 .

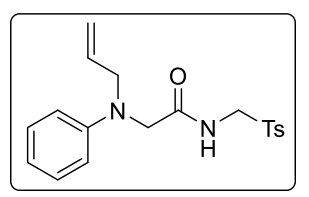

2-(Allyl(phenyl)amino)- $N$-(tosylmethyl)acetamide (3q). Purified by analytical TLC on silica gel with dichloromethane/ethyl acetate $=10: 1$ as an eluent with $\mathrm{R}_{\mathrm{f}}=0.4$, white solid (55.9 mg, 78\%), mp $=108-109^{\circ} \mathrm{C} .{ }^{1} \mathrm{H}$ NMR $\left(600 \mathrm{MHz}, \mathrm{CDCl}_{3}\right) \delta 7.69(\mathrm{~d}, J=8.2 \mathrm{~Hz}, 2 \mathrm{H}), 7.32-7.31(\mathrm{~m}, 3 \mathrm{H}), 7.26(\mathrm{t}, J$ $=7.9 \mathrm{~Hz}, 2 \mathrm{H}), 6.87(\mathrm{t}, J=7.3 \mathrm{~Hz}, 1 \mathrm{H}), 6.67(\mathrm{~d}, J=8.3 \mathrm{~Hz}, 2 \mathrm{H}), 5.88-5.82(\mathrm{~m}, 1 \mathrm{H}), 5.24(\mathrm{~d}, J=$ $10.3 \mathrm{~Hz}, 1 \mathrm{H}), 5.16$ (d, $J=17.2 \mathrm{~Hz}, 1 \mathrm{H}), 4.66(\mathrm{~d}, J=6.9 \mathrm{~Hz}, 2 \mathrm{H}), 3.94$ (d, $J=5.4 \mathrm{~Hz}, 2 \mathrm{H}), 3.77$ (s, 2H), 2.45 (s, 3H). ${ }^{13} \mathrm{C}\left\{{ }^{1} \mathrm{H}\right\}$ NMR (151 MHz, $\left.\mathrm{CDCl}_{3}\right) \delta 170.2,147.7,145.5,133.8,132.3,129.9$, $129.5,128.8,119.3,118.5,113.7,59.8,55.4,54.4,21.8$. HRMS (ESI) $m / z:(\mathrm{M}+\mathrm{H})^{+}$calcd for $\mathrm{C}_{19} \mathrm{H}_{23} \mathrm{~N}_{2} \mathrm{O}_{3} \mathrm{~S}^{+}$, 359.1424; found, 359.1423 . 


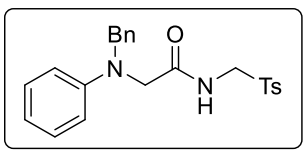

2-(Benzyl(phenyl)amino)- $N$-(tosylmethyl)acetamide (3r). Purified by analytical TLC on silica gel with dichloromethane/ethyl acetate $=10: 1$ as an eluent with $\mathrm{R}_{\mathrm{f}}=0.3$, white solid ( $35.9 \mathrm{mg}, 44 \%$ ). The spectroscopic data correspond to those previously reported in the literature. ${ }^{4}{ }^{1} \mathrm{H}$ NMR $(600$ $7.3 \mathrm{~Hz}, 2 \mathrm{H}), 7.15(\mathrm{t}, J=6.6 \mathrm{~Hz}, 1 \mathrm{H}), 6.89(\mathrm{t}, J=7.3 \mathrm{~Hz}, 1 \mathrm{H}), 6.73(\mathrm{~d}, J=8.1 \mathrm{~Hz}, 2 \mathrm{H}), 4.60$ (d, $J=$ $6.8 \mathrm{~Hz}, 2 \mathrm{H}), 4.56(\mathrm{~s}, 2 \mathrm{H}), 3.83(\mathrm{~s}, 2 \mathrm{H}), 2.43(\mathrm{~s}, 3 \mathrm{H}) .{ }^{13} \mathrm{C}\left\{{ }^{1} \mathrm{H}\right\} \mathrm{NMR}\left(151 \mathrm{MHz}, \mathrm{CDCl}_{3}\right) \delta 169.9$, 148.0, 145.4, 137.0, 133.7, 129.9, 129.6, 129.0, 128.8, 127.6, 127.2, 119.5, 113.8, 59.8, 56.0, 55.4, 21.7. HRMS (ESI) $m / z$ : $(\mathrm{M}+\mathrm{H})^{+}$calcd for $\mathrm{C}_{23} \mathrm{H}_{25} \mathrm{~N}_{2} \mathrm{O}_{3} \mathrm{~S}^{+}$, 409.1581; found, 409.1581 .

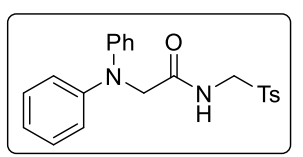

2-(Diphenylamino)- $N$-(tosylmethyl)acetamide (3s). Purified by analytical TLC on silica gel with dichloromethane/ethyl acetate $=10: 1$ as an eluent with $\mathrm{R}_{\mathrm{f}}=0.5$, white solid $(26.8 \mathrm{mg}, 34 \%)$. The spectroscopic data correspond to those previously reported in the literature. ${ }^{4}{ }^{1} \mathrm{H}$ NMR $\left(600 \mathrm{MHz}, \mathrm{CDCl}_{3}\right) \delta$ $7.50-7.49(\mathrm{~m}, 2 \mathrm{H}), 7.35-7.33(\mathrm{~m}, 4 \mathrm{H}), 7.28(\mathrm{t}, J=6.2 \mathrm{~Hz}, 1 \mathrm{H}), 7.18(\mathrm{~d}, J=7.9 \mathrm{~Hz}, 2 \mathrm{H}), 7.11-$ $7.08(\mathrm{~m}, 2 \mathrm{H}), 6.97-6.95(\mathrm{~m}, 4 \mathrm{H}), 4.67(\mathrm{~d}, J=6.8 \mathrm{~Hz}, 2 \mathrm{H}), 4.22(\mathrm{~s}, 2 \mathrm{H}), 2.40(\mathrm{~s}, 3 \mathrm{H}) .{ }^{13} \mathrm{C}\left\{{ }^{1} \mathrm{H}\right\}$ $\operatorname{NMR}\left(101 \mathrm{MHz}, \mathrm{CDCl}_{3}\right) \delta 169.9,147.2,145.4,133.4,129.9,129.8,128.8,123.2,120.8,59.8,57.2$, 21.8. HRMS (ESI) $m / z$ : $(\mathrm{M}+\mathrm{H})^{+}$calcd for $\mathrm{C}_{22} \mathrm{H}_{23} \mathrm{~N}_{2} \mathrm{O}_{3} \mathrm{~S}^{+}$, 395.1424; found, 395.1428 .

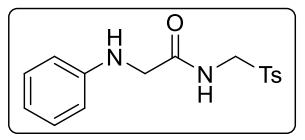

2-(Phenylamino)- $N$-(tosylmethyl)acetamide (3t). Purified by analytical TLC on silica gel with petroleum/ethyl acetate $=1: 2$ as an eluent with $\mathrm{R}_{\mathrm{f}}=$ 0.4 , white solid (33.1 mg, 52\%), mp $=144-145{ }^{\circ} \mathrm{C} .{ }^{1} \mathrm{H}$ NMR $(600 \mathrm{MHz}$, DMSO) $\delta 8.82(\mathrm{t}, J=6.6 \mathrm{~Hz}, 1 \mathrm{H}), 7.63(\mathrm{~d}, J=8.2 \mathrm{~Hz}, 2 \mathrm{H}), 7.36(\mathrm{~d}, J=8.0 \mathrm{~Hz}, 2 \mathrm{H}), 7.09$ (t, $J=7.9$ $\mathrm{Hz}, 2 \mathrm{H}), 6.60(\mathrm{t}, J=7.3 \mathrm{~Hz}, 1 \mathrm{H}), 6.45(\mathrm{~d}, J=7.8 \mathrm{~Hz}, 2 \mathrm{H}), 5.89(\mathrm{t}, J=5.8 \mathrm{~Hz}, 1 \mathrm{H}), 4.68(\mathrm{~d}, J=6.7$ $\mathrm{Hz}, 2 \mathrm{H}), 3.61(\mathrm{~d}, J=5.7 \mathrm{~Hz}, 2 \mathrm{H}), 2.39(\mathrm{~s}, 3 \mathrm{H}) .{ }^{13} \mathrm{C}\left\{{ }^{1} \mathrm{H}\right\}(151 \mathrm{MHz}, \mathrm{DMSO}) \delta 171.2,148.5,144.9$, 135.1, 130.2, 129.3, 128.9, 117.0, 112.8, 60.6, 46.8, 21.6. HRMS (ESI) $m / z:(\mathrm{M}+\mathrm{H})^{+}$calcd for $\mathrm{C}_{16} \mathrm{H}_{19} \mathrm{~N}_{2} \mathrm{O}_{3} \mathrm{~S}^{+}, 319.1111$; found, 319.1111 .

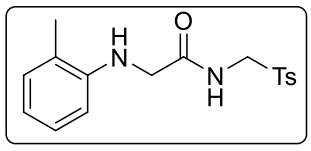

2-(o-Tolylamino)- $N$-(tosylmethyl)acetamide (3u). Purified by analytical TLC on silica gel with petroleum/ethyl acetate $=1: 2$ as an eluent with $\mathrm{R}_{\mathrm{f}}=$ 0.4 , yellow oil (34.6 mg, 52\%). ${ }^{1} \mathrm{H}$ NMR (600 MHz, DMSO) $\delta 8.80(\mathrm{t}, J=$ $6.7 \mathrm{~Hz}, 1 \mathrm{H}), 7.62(\mathrm{~d}, J=8.2 \mathrm{~Hz}, 2 \mathrm{H}), 7.37$ (d, $J=8.0 \mathrm{~Hz}, 2 \mathrm{H}), 7.01-6.99(\mathrm{~m}, 2 \mathrm{H}), 6.57$ (t, $J=7.2$ $\mathrm{Hz}, 1 \mathrm{H}), 6.19(\mathrm{~d}, J=7.9 \mathrm{~Hz}, 1 \mathrm{H}), 5.23(\mathrm{br}, 1 \mathrm{H}), 4.68(\mathrm{~d}, J=6.7 \mathrm{~Hz}, 2 \mathrm{H}), 3.66(\mathrm{~s}, 2 \mathrm{H}), 2.39$ (s, 3H), 2.09 (s, 3H). ${ }^{13} \mathrm{C}\left\{{ }^{1} \mathrm{H}\right\}(151 \mathrm{MHz}, \mathrm{DMSO}) \delta 171.3,146.2,145.0,135.1,130.2,130.1,128.9,127.2$, 122.4, 117.0, 109.7, 60.6, 47.1, 21.6, 17.9. HRMS (ESI) $\mathrm{m} / \mathrm{z}:(\mathrm{M}+\mathrm{H})^{+}$calcd for $\mathrm{C}_{17} \mathrm{H}_{21} \mathrm{~N}_{2} \mathrm{O}_{3} \mathrm{~S}^{+}$, 333.1268; found, 333.1264 .

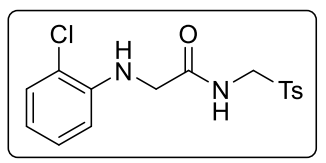

2-((2-Chlorophenyl)amino)- $N$-(tosylmethyl)acetamide (3v). Purified by analytical TLC on silica gel with petroleum/ethyl acetate $=1: 2$ as an eluent with $\mathrm{R}_{\mathrm{f}}=0.5$, white solid (31.1 mg, 44\%), $\mathrm{mp}=135-136{ }^{\circ} \mathrm{C} .{ }^{1} \mathrm{H}$ NMR $(600$ MHz, DMSO) $\delta 8.95(\mathrm{t}, J=6.6 \mathrm{~Hz}, 1 \mathrm{H}), 7.66(\mathrm{~d}, J=8.2 \mathrm{~Hz}, 2 \mathrm{H}), 7.38(\mathrm{~d}$, $J=8.1 \mathrm{~Hz}, 2 \mathrm{H}), 7.27(\mathrm{dd}, J=7.9,1.2 \mathrm{~Hz}, 1 \mathrm{H}), 7.13-7.11(\mathrm{~m}, 1 \mathrm{H}), 6.64(\mathrm{td}, J=7.7,1.2 \mathrm{~Hz}, 1 \mathrm{H})$, $6.34(\mathrm{dd}, J=8.2,0.9 \mathrm{~Hz}, 1 \mathrm{H}), 5.64(\mathrm{t}, J=5.9 \mathrm{~Hz}, 1 \mathrm{H}), 4.70(\mathrm{~d}, J=6.6 \mathrm{~Hz}, 2 \mathrm{H}), 3.74(\mathrm{~d}, J=6.0 \mathrm{~Hz}$, $2 \mathrm{H}), 2.39$ (s, 3H). ${ }^{13} \mathrm{C}\left\{{ }^{1} \mathrm{H}\right\}$ NMR (151 MHz, DMSO) $\delta 170.4,145.0,144.0,135.0,130.2,129.4$, 128.9, 128.4, 118.4, 117.6, 111.8, 60.6, 46.4, 21.6. HRMS (ESI) $m / z:(\mathrm{M}+\mathrm{H})^{+}$calcd for 


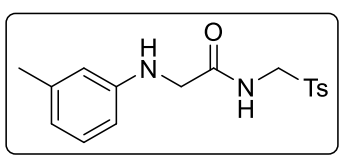

2-(m-Tolylamino)- $N$-(tosylmethyl)acetamide (3w). Purified by analytical TLC on silica gel with petroleum/ethyl acetate $=1: 2$ as an eluent with $\mathrm{R}_{\mathrm{f}}=0.3$, white solid (33.9 mg, 51\%), $\mathrm{mp}=116-117{ }^{\circ} \mathrm{C} .{ }^{1} \mathrm{H}$ NMR (600 MHz, DMSO) $\delta 8.77(\mathrm{t}, J=6.7 \mathrm{~Hz}, 1 \mathrm{H}), 7.61(\mathrm{~d}, J=8.2 \mathrm{~Hz}, 2 \mathrm{H}), 7.34(\mathrm{~d}, J=8.1 \mathrm{~Hz}$, $2 \mathrm{H}), 6.98(\mathrm{t}, J=7.7 \mathrm{~Hz}, 1 \mathrm{H}), 6.44(\mathrm{~d}, J=7.4 \mathrm{~Hz}, 1 \mathrm{H}), 6.32(\mathrm{~s}, 1 \mathrm{H}), 6.25(\mathrm{dd}, J=8.0,1.7 \mathrm{~Hz}, 1 \mathrm{H})$, $5.81(\mathrm{t}, J=6.1 \mathrm{~Hz}, 1 \mathrm{H}), 4.67(\mathrm{~d}, J=6.7 \mathrm{~Hz}, 2 \mathrm{H}), 3.60(\mathrm{~d}, J=6.2 \mathrm{~Hz}, 2 \mathrm{H}), 2.38(\mathrm{~s}, 3 \mathrm{H}), 2.19(\mathrm{~s}, 3 \mathrm{H})$. ${ }^{13} \mathrm{C}\left\{{ }^{1} \mathrm{H}\right\}$ NMR (151 MHz, DMSO) $\delta 171.3,148.5,144.9,138.3,135.2,130.1,129.2,128.9,118.0$, 113.5, 110.0, 60.6, 46.9, 21.8, 21.6. HRMS (ESI) $m / z:(\mathrm{M}+\mathrm{H})^{+}$calcd for $\mathrm{C}_{17} \mathrm{H}_{21} \mathrm{~N}_{2} \mathrm{O}_{3} \mathrm{~S}^{+}, 333.1268$; found, 333.1265 .

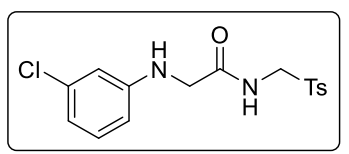

2-((3-Chlorophenyl)amino)- $N$-(tosylmethyl)acetamide (3x). Purified by analytical TLC on silica gel with petroleum/ethyl acetate $=1: 2$ as an eluent with $\mathrm{R}_{\mathrm{f}}=0.3$, white solid (32.5 mg, $46 \%$ ), $\mathrm{mp}=136-137{ }^{\circ} \mathrm{C} .{ }^{1} \mathrm{H}$ NMR (600 MHz, DMSO) $\delta 8.90(\mathrm{t}, J=6.6 \mathrm{~Hz}, 1 \mathrm{H}), 7.65(\mathrm{~d}, J=8.2 \mathrm{~Hz}$, 2H), $7.36(\mathrm{~d}, J=8.1 \mathrm{~Hz}, 2 \mathrm{H}), 7.09$ (t, $J=8.0 \mathrm{~Hz}, 1 \mathrm{H}), 6.60(\mathrm{dd}, J=7.8,1.3 \mathrm{~Hz}, 1 \mathrm{H}), 6.49$ (t, $J=$ $2.0 \mathrm{~Hz}, 1 \mathrm{H}), 6.40(\mathrm{dd}, J=8.2,1.8 \mathrm{~Hz}, 1 \mathrm{H}), 6.25(\mathrm{t}, J=6.2 \mathrm{~Hz}, 1 \mathrm{H}), 4.68(\mathrm{~d}, J=6.7 \mathrm{~Hz}, 2 \mathrm{H}), 3.64$ $(\mathrm{d}, J=6.2 \mathrm{~Hz}, 2 \mathrm{H}), 2.38(\mathrm{~s}, 3 \mathrm{H}) .{ }^{13} \mathrm{C}\left\{{ }^{1} \mathrm{H}\right\} \mathrm{NMR}(151 \mathrm{MHz}, \mathrm{DMSO}) \delta 170.7,150.1,145.0,135.1$, 134.0, 130.8, 130.2, 128.9, 116.3, 112.0, 111.4, 60.6, 46.3, 21.6. HRMS (ESI) $\mathrm{m} / \mathrm{z}:(\mathrm{M}+\mathrm{H})^{+}$calcd for $\mathrm{C}_{16} \mathrm{H}_{18} \mathrm{ClN}_{2} \mathrm{O}_{3} \mathrm{~S}^{+}$, 353.0721; found, 353.0719.

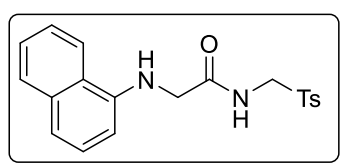

2-(Naphthalen-1-ylamino)- $N$-(tosylmethyl)acetamide (3y). Purified by analytical TLC on silica gel with petroleum/ethyl acetate $=1: 2$ as an eluent with $\mathrm{R}_{\mathrm{f}}=0.3$, white solid (31.7 mg, 43\%), $\mathrm{mp}=134-135{ }^{\circ} \mathrm{C}$. ${ }^{1} \mathrm{H}$ NMR (600 MHz, DMSO) $\delta 8.87(\mathrm{t}, J=6.7 \mathrm{~Hz}, 1 \mathrm{H}), 8.09(\mathrm{~d}, J=8.3 \mathrm{~Hz}, 1 \mathrm{H})$, $7.80(\mathrm{~d}, J=7.7 \mathrm{~Hz}, 1 \mathrm{H}), 7.58(\mathrm{~d}, J=8.2 \mathrm{~Hz}, 2 \mathrm{H}), 7.48-7.41(\mathrm{~m}, 2 \mathrm{H}), 7.30-7.25(\mathrm{~m}, 3 \mathrm{H}), 7.19(\mathrm{~d}$, $J=8.1 \mathrm{~Hz}, 1 \mathrm{H}), 6.59$ (t, $J=6.0 \mathrm{~Hz}, 1 \mathrm{H}), 6.20(\mathrm{~d}, J=7.5 \mathrm{~Hz}, 1 \mathrm{H}), 4.69(\mathrm{~d}, J=6.7 \mathrm{~Hz}, 2 \mathrm{H}), 3.83(\mathrm{~d}$, $J=5.9 \mathrm{~Hz}, 2 \mathrm{H}), 2.33(\mathrm{~s}, 3 \mathrm{H}) .{ }^{13} \mathrm{C}\left\{{ }^{1} \mathrm{H}\right\}$ NMR (151 MHz, DMSO) $\delta 171.1,144.9,143.8,135.1,134.4$, 130.1, 128.9, 128.4, 127.1, 126.2, 124.6, 123.5, 122.1, 116.8, 103.8, 60.7, 47.1, 21.5. HRMS (ESI) $m / z:(\mathrm{M}+\mathrm{H})^{+}$calcd for $\mathrm{C}_{20} \mathrm{H}_{21} \mathrm{~N}_{2} \mathrm{O}_{3} \mathrm{~S}^{+}, 369.1268$; found, 369.1264 .

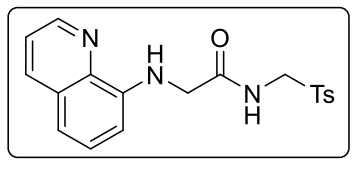

2-(Quinolin-8-ylamino)-N-(tosylmethyl)acetamide (3z). Purified by analytical TLC on silica gel with petroleum/ethyl acetate $=1: 2$ as an eluent with $\mathrm{R}_{\mathrm{f}}=0.3$, white solid (26.6 mg, 36\%), $\mathrm{mp}=156-157^{\circ} \mathrm{C} .{ }^{1} \mathrm{H}$ NMR (600 MHz, DMSO) $\delta 8.99(\mathrm{t}, J=6.6 \mathrm{~Hz}, 1 \mathrm{H}), 8.76(\mathrm{dd}, J=4.1$, $1.5 \mathrm{~Hz}, 1 \mathrm{H}), 8.25(\mathrm{dd}, J=8.2,1.4 \mathrm{~Hz}, 1 \mathrm{H}), 7.63(\mathrm{~d}, J=8.2 \mathrm{~Hz}, 2 \mathrm{H}), 7.53(\mathrm{dd}, J=8.2,4.1 \mathrm{~Hz}, 1 \mathrm{H})$, $7.38(\mathrm{t}, J=7.9 \mathrm{~Hz}, 1 \mathrm{H}), 7.28(\mathrm{~d}, J=8.0 \mathrm{~Hz}, 2 \mathrm{H}), 7.15(\mathrm{~d}, J=8.0 \mathrm{~Hz}, 1 \mathrm{H}), 6.85(\mathrm{~s}, 1 \mathrm{H}), 6.40(\mathrm{~d}, J=$ $7.5 \mathrm{~Hz}, 1 \mathrm{H}), 4.71(\mathrm{~d}, J=6.6 \mathrm{~Hz}, 2 \mathrm{H}), 3.89(\mathrm{~s}, 2 \mathrm{H}), 2.33(\mathrm{~s}, 3 \mathrm{H}) .{ }^{13} \mathrm{C}\left\{{ }^{1} \mathrm{H}\right\} \mathrm{NMR}(151 \mathrm{MHz}, \mathrm{DMSO})$ $\delta 170.5,147.6,144.9,144.3,138.0,136.4,135.0,130.1,128.9,128.7,128.1,122.3,114.6,105.2$, 60.6, 46.5, 21.5. HRMS (ESI) $\mathrm{m} / \mathrm{z}$ : $(\mathrm{M}+\mathrm{H})^{+}$calcd for $\mathrm{C}_{19} \mathrm{H}_{20} \mathrm{~N}_{3} \mathrm{O}_{3} \mathrm{~S}^{+}$, 370.1220; found, 370.1219. 


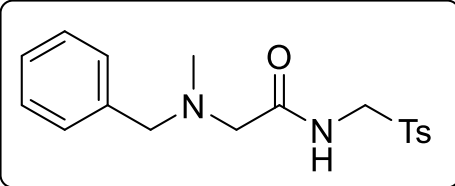

2-(Benzyl(methyl)amino)- $N$-(tosylmethyl)acetamide (3aa).

Purified by analytical TLC on silica gel with dichloromethane/ethyl acetate $=10: 1$ as an eluent with $\mathrm{R}_{\mathrm{f}}=0.3$, white solid (34.0 mg, 49\%), mp = 89-90 ${ }^{\circ} \mathrm{C} .{ }^{1} \mathrm{H}$ NMR $(600 \mathrm{MHz}$, $\left.\mathrm{CDCl}_{3}\right) \delta 7.97(\mathrm{br}, 1 \mathrm{H}), 7.61(\mathrm{~d}, J=8.2 \mathrm{~Hz}, 2 \mathrm{H}), 7.40(\mathrm{t}, J=7.3 \mathrm{~Hz}, 2 \mathrm{H}), 7.35-7.32$ (m, 3H), 7.19 $(\mathrm{d}, J=8.0 \mathrm{~Hz}, 2 \mathrm{H}), 4.66(\mathrm{~d}, J=7.0 \mathrm{~Hz}, 2 \mathrm{H}), 3.54(\mathrm{~s}, 2 \mathrm{H}), 2.88(\mathrm{~s}, 2 \mathrm{H}), 2.39$ (s, 3H), 2.30 (s, 3H). ${ }^{13} \mathrm{C}\left\{{ }^{1} \mathrm{H}\right\} \mathrm{NMR}\left(101 \mathrm{MHz}, \mathrm{CDCl}_{3}\right) \delta 170.3,145.3,137.5,133.7,129.8,129.2,128.74,128.71,127.8$, 62.3, 59.8, 59.3, 43.7, 21.7. HRMS (ESI) $m / z$ : $(\mathrm{M}+\mathrm{H})^{+}$calcd for $\mathrm{C}_{18} \mathrm{H}_{23} \mathrm{~N}_{2} \mathrm{O}_{3} \mathrm{~S}^{+}, 347.1424$; found, 347.1461 .

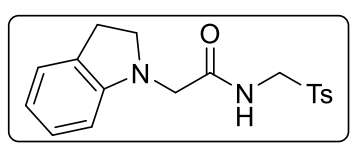

2-(Indolin-1-yl)- $N$-(tosylmethyl)acetamide (5a). Purified by analytical TLC on silica gel with dichloromethane/ethyl acetate $=10: 1$ as an eluent with $\mathrm{R}_{\mathrm{f}}=0.5$, yellow oil (37.9 $\left.\mathrm{mg}, 55 \%\right) .{ }^{1} \mathrm{H} \mathrm{NMR}\left(400 \mathrm{MHz}, \mathrm{CDCl}_{3}\right) \delta$ $7.75(\mathrm{~d}, J=8.3 \mathrm{~Hz}, 2 \mathrm{H}), 7.67(\mathrm{t}, J=6.7 \mathrm{~Hz}, 1 \mathrm{H}), 7.34(\mathrm{~d}, J=8.0 \mathrm{~Hz}, 2 \mathrm{H}), 7.15(\mathrm{~d}, J=7.2 \mathrm{~Hz}, 1 \mathrm{H})$, $7.10(\mathrm{t}, J=7.7 \mathrm{~Hz}, 1 \mathrm{H}), 6.81(\mathrm{dt}, J=7.4,0.7 \mathrm{~Hz}, 1 \mathrm{H}), 6.33(\mathrm{~d}, J=7.8 \mathrm{~Hz}, 1 \mathrm{H}), 4.69$ (d, $J=7.0 \mathrm{~Hz}$, 2H), 3.55 (s, 2H), 3.40 (t, $J=8.2 \mathrm{~Hz}, 2 \mathrm{H}), 3.04(\mathrm{t}, J=8.2 \mathrm{~Hz}, 2 \mathrm{H}), 2.45(\mathrm{~s}, 3 \mathrm{H}) \cdot{ }^{13} \mathrm{C}\left\{{ }^{1} \mathrm{H}\right\} \mathrm{NMR}(101$ $\left.\mathrm{MHz}_{\mathrm{CDCl}}\right) \delta 170.0,151.3,145.6,133.8,130.0,129.9,128.9,127.5,124.9,119.9,107.7,59.9$, 55.6, 54.9, 28.8, 21.8. HRMS (ESI) $m / z$ : $(\mathrm{M}+\mathrm{H})^{+}$calcd for $\mathrm{C}_{18} \mathrm{H}_{21} \mathrm{~N}_{2} \mathrm{O}_{3} \mathrm{~S}^{+}, 345.1268$; found, 345.1271 .

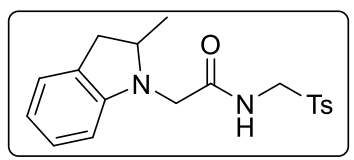

2-(2-Methylindolin-1-yl)-N-(tosylmethyl)acetamide (5b). Purified by analytical TLC on silica gel with dichloromethane/ethyl acetate $=10: 1$ as an eluent with $\mathrm{R}_{\mathrm{f}}=0.5$, yellow oil $(42.3 \mathrm{mg}, 59 \%) .{ }^{1} \mathrm{H} \mathrm{NMR}(600 \mathrm{MHz}$, $\left.\mathrm{CDCl}_{3}\right) \delta 7.74(\mathrm{t}, J=8.1 \mathrm{~Hz}, 2 \mathrm{H}), 7.65(\mathrm{br}, 1 \mathrm{H}), 7.32(\mathrm{~d}, J=8.0 \mathrm{~Hz}, 2 \mathrm{H})$, $7.11-7.03(\mathrm{~m}, 2 \mathrm{H}), 6.81(\mathrm{t}, J=7.4 \mathrm{~Hz}, 1 \mathrm{H}), 6.24(\mathrm{~d}, J=7.8 \mathrm{~Hz}, 1 \mathrm{H}), 4.81-4.78(\mathrm{~m}, 1 \mathrm{H}), 4.60-$ 4.57 (m, 1H), $3.61-3.43(\mathrm{~m}, 3 \mathrm{H}), 3.18-3.14(\mathrm{~m}, 1 \mathrm{H}), 2.73-2.69(\mathrm{~m}, 1 \mathrm{H}), 2.46(\mathrm{~s}, 3 \mathrm{H}), 1.36(\mathrm{~d}$, $J=6.1 \mathrm{~Hz}, 3 \mathrm{H}) .{ }^{13} \mathrm{C}\left\{{ }^{1} \mathrm{H}\right\} \mathrm{NMR}\left(151 \mathrm{MHz}, \mathrm{CDCl}_{3}\right) \delta 170.3,151.6,145.5,133.8,129.9,129.3,128.9$, 127.6, 124.5, 120.1, 107.7, 63.0, 59.8, 53.1, 37.2, 21.8, 19.5. HRMS (ESI) $m / z:(\mathrm{M}+\mathrm{H})^{+}$calcd for $\mathrm{C}_{19} \mathrm{H}_{23} \mathrm{~N}_{2} \mathrm{O}_{3} \mathrm{~S}^{+}, 359.1424$; found, 359.1432.

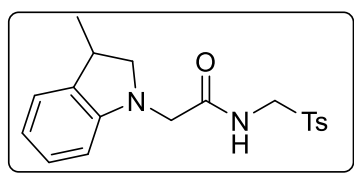

2-(3-Methylindolin-1-yl)- $N$-(tosylmethyl)acetamide (5c). Purified by analytical TLC on silica gel with dichloromethane/ethyl acetate $=10: 1$ as an eluent with $\mathrm{R}_{\mathrm{f}}=0.5$, yellow oil $(35.8 \mathrm{mg}, 50 \%) .{ }^{1} \mathrm{H}$ NMR $(600$ $\left.\mathrm{MHz} \mathrm{CDCl}_{3}\right) \delta 7.75(\mathrm{~d}, J=8.2 \mathrm{~Hz}, 2 \mathrm{H}), 7.60(\mathrm{t}, J=6.5 \mathrm{~Hz}, 1 \mathrm{H}), 7.34$ $(\mathrm{d}, J=8.1 \mathrm{~Hz}, 2 \mathrm{H}), 7.13-7.11(\mathrm{~m}, 2 \mathrm{H}), 6.86(\mathrm{t}, J=7.4 \mathrm{~Hz}, 1 \mathrm{H}), 6.35(\mathrm{~d}, J=7.7 \mathrm{~Hz}, 1 \mathrm{H}), 4.74-$ $4.66(\mathrm{~m}, 2 \mathrm{H}), 3.66-3.45(\mathrm{~m}, 3 \mathrm{H}), 3.40-3.34(\mathrm{~m}, 1 \mathrm{H}), 2.88(\mathrm{t}, J=8.6 \mathrm{~Hz}, 1 \mathrm{H}), 2.45(\mathrm{~s}, 3 \mathrm{H}), 1.35$ $(\mathrm{d}, J=6.8 \mathrm{~Hz}, 3 \mathrm{H}) .{ }^{13} \mathrm{C}\left\{{ }^{1} \mathrm{H}\right\} \mathrm{NMR}\left(151 \mathrm{MHz}, \mathrm{CDCl}_{3}\right) \delta 170.0,150.8,145.6,134.9,133.8,130.0$, 128.8, 127.6, 123.5, 120.1, 107.7, 63.5, 59.8, 54.7, 35.5, 21.8, 18.2. HRMS (ESI) $m / z:(\mathrm{M}+\mathrm{H})^{+}$ calcd for $\mathrm{C}_{19} \mathrm{H}_{23} \mathrm{~N}_{2} \mathrm{O}_{3} \mathrm{~S}^{+}, 359.1424$; found, 359.1422 .

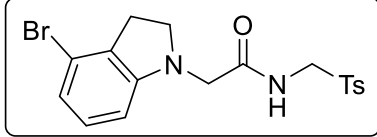

2-(4-Bromoindolin-1-yl)- $N$-(tosylmethyl)acetamide (5d). Purified by analytical TLC on silica gel with dichloromethane/ethyl acetate $=$ 10:1 as an eluent with $\mathrm{R}_{\mathrm{f}}=0.4$, white solid $(60.1 \mathrm{mg}, 71 \%), \mathrm{mp}=$ $145-146{ }^{\circ} \mathrm{C} .{ }^{1} \mathrm{H}$ NMR $\left(600 \mathrm{MHz}, \mathrm{CDCl}_{3}\right) \delta 7.74(\mathrm{~d}, J=8.2 \mathrm{~Hz}, 2 \mathrm{H}), 7.51(\mathrm{t}, J=6.7 \mathrm{~Hz}, 1 \mathrm{H}), 7.34$ $(\mathrm{d}, J=8.0 \mathrm{~Hz}, 2 \mathrm{H}), 6.98-6.93(\mathrm{~m}, 2 \mathrm{H}), 6.24(\mathrm{~d}, J=7.4 \mathrm{~Hz}, 1 \mathrm{H}), 4.69(\mathrm{~d}, J=6.9 \mathrm{~Hz}, 2 \mathrm{H}), 3.56(\mathrm{~s}$, 
2H), $3.46(\mathrm{t}, J=8.3 \mathrm{~Hz}, 2 \mathrm{H}), 3.05(\mathrm{t}, J=8.3 \mathrm{~Hz}, 2 \mathrm{H}), 2.46(\mathrm{~s}, 3 \mathrm{H}) .{ }^{13} \mathrm{C}\left\{{ }^{1} \mathrm{H}\right\} \mathrm{NMR}\left(151 \mathrm{MHz}, \mathrm{CDCl}_{3}\right)$ $\delta$ 169.4, 152.2, 145.6, 133.8, 130.4, 130.0, 129.2, 128.8, 122.7, 119.9, 106.2, 59.9, 54.6, 54.5, 30.1, 21.8. HRMS (ESI) $m / z$ : $(\mathrm{M}+\mathrm{H})^{+}$calcd for $\mathrm{C}_{18} \mathrm{H}_{20} \mathrm{BrN}_{2} \mathrm{O}_{3} \mathrm{~S}^{+}$, 423.0373; found, 423.0374 .

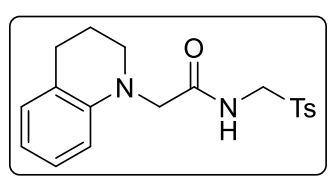

\section{2-(3,4-Dihydroquinolin-1(2H)-yl)- $N$-(tosylmethyl)acetamide}

(5f).

Purified by analytical TLC on silica gel with dichloromethane/ethyl acetate

$=10: 1$ as an eluent with $\mathrm{R}_{\mathrm{f}}=0.4$, white solid $(59.5 \mathrm{mg}, 83 \%), \mathrm{mp}=$ 130-131 ${ }^{\circ} \mathrm{C} .{ }^{1} \mathrm{H}$ NMR $\left(600 \mathrm{MHz}, \mathrm{CDCl}_{3}\right) \delta 7.71(\mathrm{~d}, J=8.2 \mathrm{~Hz}, 2 \mathrm{H}), 7.33$ $-7.32(\mathrm{~m}, 3 \mathrm{H}), 7.06(\mathrm{t}, J=7.7 \mathrm{~Hz}, 1 \mathrm{H}), 7.02(\mathrm{~d}, J=7.6 \mathrm{~Hz}, 1 \mathrm{H}), 6.75(\mathrm{t}, J=7.3 \mathrm{~Hz}, 1 \mathrm{H}), 6.30(\mathrm{~d}$, $J=8.2 \mathrm{~Hz}, 1 \mathrm{H}), 4.66(\mathrm{~d}, J=6.9 \mathrm{~Hz}, 2 \mathrm{H}), 3.69(\mathrm{~s}, 2 \mathrm{H}), 3.26(\mathrm{t}, J=5.6 \mathrm{~Hz}, 2 \mathrm{H}), 2.82(\mathrm{t}, J=6.4 \mathrm{~Hz}$, 2H), $2.45(\mathrm{~s}, 3 \mathrm{H}), 2.06-2.02(\mathrm{~m}, 2 \mathrm{H}) .{ }^{13} \mathrm{C}\left\{{ }^{1} \mathrm{H}\right\} \mathrm{NMR}\left(101 \mathrm{MHz}, \mathrm{CDCl}_{3}\right) \delta 170.3,145.5,144.8$, $133.7,130.0,129.6,128.8,127.4,123.6,118.8,112.1,59.8,57.3,51.5,27.5,22.5,21.8$. HRMS (ESI) $m / z:(\mathrm{M}+\mathrm{H})^{+}$calcd for $\mathrm{C}_{19} \mathrm{H}_{23} \mathrm{~N}_{2} \mathrm{O}_{3} \mathrm{~S}^{+}, 359.1424$; found, 359.1421 .

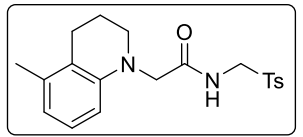

2-(5-Methyl-3,4-dihydroquinolin-1(2H)-yl)- $N$-(tosylmethyl)acetamide (5g). Purified by analytical TLC on silica gel with dichloromethane/ethyl acetate $=10: 1$ as an eluent with $\mathrm{R}_{\mathrm{f}}=0.3$, white solid $(55.9 \mathrm{mg}, 75 \%), \mathrm{mp}=$ 139-140 ${ }^{\circ} \mathrm{C} .{ }^{1} \mathrm{H}$ NMR $\left(600 \mathrm{MHz}, \mathrm{CDCl}_{3}\right) \delta 7.71(\mathrm{~d}, J=8.0 \mathrm{~Hz}, 2 \mathrm{H}), 7.42(\mathrm{t}$, $J=6.1 \mathrm{~Hz}, 1 \mathrm{H}), 7.32(\mathrm{~d}, J=8.0 \mathrm{~Hz}, 2 \mathrm{H}), 6.97(\mathrm{t}, J=7.8 \mathrm{~Hz}, 1 \mathrm{H}), 6.66(\mathrm{~d}, J=7.4 \mathrm{~Hz}, 1 \mathrm{H}), 6.19(\mathrm{~d}$, $J=8.3 \mathrm{~Hz}, 1 \mathrm{H}), 4.65(\mathrm{~d}, J=6.9 \mathrm{~Hz}, 2 \mathrm{H}), 3.66(\mathrm{~s}, 2 \mathrm{H}), 3.22(\mathrm{t}, J=5.4 \mathrm{~Hz}, 2 \mathrm{H}), 2.68(\mathrm{t}, J=6.5 \mathrm{~Hz}$, $2 \mathrm{H}), 2.45(\mathrm{~s}, 3 \mathrm{H}), 2.21(\mathrm{~s}, 3 \mathrm{H}), 2.08-2.04(\mathrm{~m}, 2 \mathrm{H}) .{ }^{13} \mathrm{C}\left\{{ }^{1} \mathrm{H}\right\} \mathrm{NMR}\left(151 \mathrm{MHz}, \mathrm{CDCl}_{3}\right) \delta 170.3$, 145.4, 145.1, 137.2, 133.8, 129.9, 128.8, 126.6, 122.3, 120.8, 110.4, 59.8, 57.8, 51.2, 24.5, 22.5, 21.7, 19.8. HRMS (ESI) $m / z$ : $(\mathrm{M}+\mathrm{H})^{+}$calcd for $\mathrm{C}_{20} \mathrm{H}_{25} \mathrm{~N}_{2} \mathrm{O}_{3} \mathrm{~S}^{+}, 373.1581$; found, 373.1583 .

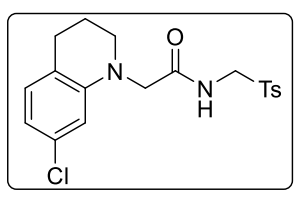

2-(7-Chloro-3,4-dihydroquinolin-1(2H)-yl)- $N$-(tosylmethyl)acetamide (5h). Purified by analytical TLC on silica gel with dichloromethane/ethyl acetate $=10: 1$ as an eluent with $\mathrm{R}_{\mathrm{f}}=0.3$, white solid $(60.5 \mathrm{mg}, 77 \%), \mathrm{mp}=$ 163-164 ${ }^{\circ} \mathrm{C} .{ }^{1} \mathrm{H}$ NMR $\left(600 \mathrm{MHz}, \mathrm{CDCl}_{3}\right) \delta 7.74(\mathrm{~d}, J=8.2 \mathrm{~Hz}, 2 \mathrm{H}), 7.37(\mathrm{~d}$, $J=8.1 \mathrm{~Hz}, 2 \mathrm{H}), 7.21(\mathrm{br}, 1 \mathrm{H}), 6.92(\mathrm{~d}, J=8.0 \mathrm{~Hz}, 1 \mathrm{H}), 6.70(\mathrm{~d}, J=8.0 \mathrm{~Hz}, 1 \mathrm{H}), 6.30(\mathrm{~d}, J=1.7$ $\mathrm{Hz}, 1 \mathrm{H}), 4.68(\mathrm{~d}, J=6.8 \mathrm{~Hz}, 2 \mathrm{H}), 3.69(\mathrm{~s}, 2 \mathrm{H}), 3.25(\mathrm{t}, J=5.6 \mathrm{~Hz}, 2 \mathrm{H}), 2.77(\mathrm{t}, J=6.4 \mathrm{~Hz}, 2 \mathrm{H})$, $2.45(\mathrm{~s}, 3 \mathrm{H}), 2.04-2.00(\mathrm{~m}, 2 \mathrm{H}) .{ }^{13} \mathrm{C}\left\{{ }^{1} \mathrm{H}\right\} \mathrm{NMR}\left(151 \mathrm{MHz}, \mathrm{CDCl}_{3}\right) \delta 169.5,145.7,145.6,133.7$, 132.8, 130.5, 130.1, 128.8, 121.8, 118.4, 111.6, 59.9, 56.7, 51.1, 27.1, 22.2, 21.8. HRMS (ESI) $\mathrm{m} / \mathrm{z}$ : $(\mathrm{M}+\mathrm{H})^{+}$calcd for $\mathrm{C}_{19} \mathrm{H}_{22} \mathrm{ClN}_{2} \mathrm{O}_{3} \mathrm{~S}^{+}, 393.1034$; found, 393.1038.

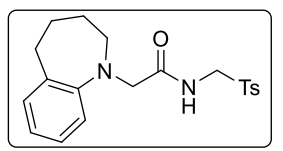

2-(2,3,4,5-Tetrahydro- $1 H$-benzo[b]azepin-1-yl)- $N$-(tosylmethyl)acetamide

(5i). Purified by analytical TLC on silica gel with dichloromethane/ethyl acetate $=10: 1$ as an eluent with $\mathrm{R}_{\mathrm{f}}=0.3$, yellow oil $(57.4 \mathrm{mg}, 77 \%) .{ }^{1} \mathrm{H}$ NMR $\left(600 \mathrm{MHz}, \mathrm{CDCl}_{3}\right) \delta 7.74(\mathrm{t}, J=6.5 \mathrm{~Hz}, 1 \mathrm{H}), 7.64(\mathrm{~d}, J=8.1 \mathrm{~Hz}, 2 \mathrm{H}), 7.29(\mathrm{~d}$, $J=7.1 \mathrm{~Hz}, 2 \mathrm{H}), 7.21-7.17(\mathrm{~m}, 2 \mathrm{H}), 7.02(\mathrm{t}, J=7.4 \mathrm{~Hz}, 1 \mathrm{H}), 6.88(\mathrm{~d}, J=8.0 \mathrm{~Hz}, 1 \mathrm{H}), 4.72(\mathrm{~d}, J=$ $6.9 \mathrm{~Hz}, 2 \mathrm{H}), 3.76(\mathrm{~s}, 2 \mathrm{H}), 2.91-2.88(\mathrm{~m}, 4 \mathrm{H}), 2.44(\mathrm{~s}, 3 \mathrm{H}), 1.91-1.87(\mathrm{~m}, 2 \mathrm{H}), 1.71-1.67(\mathrm{~m}$, 2H). ${ }^{13} \mathrm{C}\left\{{ }^{1} \mathrm{H}\right\}$ NMR $\left(151 \mathrm{MHz}, \mathrm{CDCl}_{3}\right) \delta 170.1,150.4,145.4,136.8,133.9,130.5,129.9,128.6$, $127.1,123.4,117.9,59.9,58.7,56.5,35.0,30.5,25.5,21.7$. HRMS (ESI) $m / z:(\mathrm{M}+\mathrm{H})^{+}$calcd for $\mathrm{C}_{20} \mathrm{H}_{25} \mathrm{~N}_{2} \mathrm{O}_{3} \mathrm{~S}^{+}, 373.1581$; found, 373.1586 . 


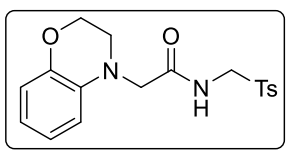

2-(2,3-Dihydro-4H-benzo[b][1,4]oxazin-4-yl)- $N$-(tosylmethyl)acetamide (5j). Purified by analytical TLC on silica gel with dichloromethane/ethyl acetate $=10: 1$ as an eluent with $\mathrm{R}_{\mathrm{f}}=0.2$, white solid (59.8 $\left.\mathrm{mg}, 83 \%\right), \mathrm{mp}=$ $133-134{ }^{\circ} \mathrm{C} .{ }^{1} \mathrm{H}$ NMR $\left(600 \mathrm{MHz}, \mathrm{CDCl}_{3}\right) \delta 7.72(\mathrm{~d}, J=8.1 \mathrm{~Hz}, 2 \mathrm{H}), 7.39(\mathrm{t}$, $J=5.9 \mathrm{~Hz}, 1 \mathrm{H}), 7.33(\mathrm{~d}, J=8.0 \mathrm{~Hz}, 2 \mathrm{H}), 6.85-6.78(\mathrm{~m}, 3 \mathrm{H}), 6.41(\mathrm{~d}, J=7.8 \mathrm{~Hz}, 1 \mathrm{H}), 4.69(\mathrm{~d}, J$ $=6.8 \mathrm{~Hz}, 2 \mathrm{H}), 4.31(\mathrm{t}, J=4.2 \mathrm{~Hz}, 2 \mathrm{H}), 3.70(\mathrm{~s}, 2 \mathrm{H}), 3.35(\mathrm{t}, J=4.2 \mathrm{~Hz}, 2 \mathrm{H}), 2.46(\mathrm{~s}, 3 \mathrm{H}) .{ }^{13} \mathrm{C}\left\{{ }^{1} \mathrm{H}\right\}$ NMR $\left(151 \mathrm{MHz}, \mathrm{CDCl}_{3}\right) \delta 169.6,145.6,144.8,134.2,133.8,130.0,128.8,121.7,120.6,116.9$, 113.6, 64.6, 59.9, 56.8, 49.4, 21.8. HRMS (ESI) $m / z:(\mathrm{M}+\mathrm{H})^{+}$calcd for $\mathrm{C}_{18} \mathrm{H}_{21} \mathrm{~N}_{2} \mathrm{O}_{4} \mathrm{~S}^{+}, 361.1217$; found, 361.1221 .

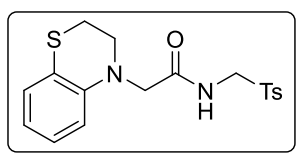

\section{2-(2,3-Dihydro-4H-benzo[b][1,4] thiazin-4-yl)- $N$-(tosylmethyl)acetamide}

(5k). Purified by analytical TLC on silica gel with dichloromethane/ethyl acetate $=10: 1$ as an eluent with $\mathrm{R}_{\mathrm{f}}=0.3$, white solid $(54.2 \mathrm{mg}, 72 \%), \mathrm{mp}=$ 159-160 ${ }^{\circ} \mathrm{C} .{ }^{1} \mathrm{H}$ NMR $\left(600 \mathrm{MHz}, \mathrm{CDCl}_{3}\right) \delta 7.68(\mathrm{~d}, J=8.1 \mathrm{~Hz}, 2 \mathrm{H}), 7.36-$ $7.31(\mathrm{~m}, 3 \mathrm{H}), 7.08(\mathrm{~d}, J=7.7 \mathrm{~Hz}, 1 \mathrm{H}), 7.00(\mathrm{t}, J=7.7 \mathrm{~Hz}, 1 \mathrm{H}), 6.76(\mathrm{t}, J=7.5 \mathrm{~Hz}, 1 \mathrm{H}), 6.44(\mathrm{~d}, J$ $=8.3 \mathrm{~Hz}, 1 \mathrm{H}), 4.66(\mathrm{~d}, J=6.9 \mathrm{~Hz}, 2 \mathrm{H}), 3.77(\mathrm{~s}, 2 \mathrm{H}), 3.57(\mathrm{t}, J=5.0 \mathrm{~Hz}, 2 \mathrm{H}), 3.09(\mathrm{t}, J=5.0 \mathrm{~Hz}$, 2H), 2.45 (s, 3H). ${ }^{13} \mathrm{C}\left\{{ }^{1} \mathrm{H}\right\}$ NMR (151 MHz, $\left.\mathrm{CDCl}_{3}\right) \delta 169.7,145.5,142.6,133.9,130.0,128.7$, 128.0, 126.1 120.0, 119.9, 114.2, 59.9, 57.7, 51.7, 25.8, 21.8. HRMS (ESI) $m / z:(\mathrm{M}+\mathrm{H})^{+}$calcd for $\mathrm{C}_{18} \mathrm{H}_{21} \mathrm{~N}_{2} \mathrm{O}_{3} \mathrm{~S}_{2}^{+}$, 377.0988; found, 377.0989 .

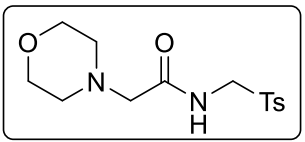

2-Morpholino- $N$-(tosylmethyl)acetamide (5I). Purified by analytical TLC on silica gel with petroleum/ethyl acetate $=1: 2$ as an eluent with $\mathrm{R}_{\mathrm{f}}=0.2$, white solid (21.9 mg, 35\%), mp $=132-133{ }^{\circ} \mathrm{C} .{ }^{1} \mathrm{H}$ NMR $\left(600 \mathrm{MHz}, \mathrm{CDCl}_{3}\right)$ $\delta 7.91(\mathrm{t}, J=6.6 \mathrm{~Hz}, 1 \mathrm{H}), 7.77(\mathrm{~d}, J=8.2 \mathrm{~Hz}, 2 \mathrm{H}), 7.35(\mathrm{~d}, J=8.0 \mathrm{~Hz}, 2 \mathrm{H}), 4.72(\mathrm{~d}, J=7.0 \mathrm{~Hz}$, 2H), $3.73(\mathrm{t}, J=4.4 \mathrm{~Hz}, 4 \mathrm{H}), 2.93(\mathrm{~s}, 2 \mathrm{H}), 2.49$ (t, $J=4.5 \mathrm{~Hz}, 4 \mathrm{H}), 2.45(\mathrm{~s}, 3 \mathrm{H}) .{ }^{13} \mathrm{C}\left\{{ }^{1} \mathrm{H}\right\}$ NMR $(151$ $\left.\mathrm{MHz}, \mathrm{CDCl}_{3}\right) \delta 169.6,145.6,134.1,130.0,128.6,66.8,61.5,59.8,53.8,21.7$. HRMS (ESI) $\mathrm{m} / \mathrm{z}$ : $(\mathrm{M}+\mathrm{H})^{+}$calcd for $\mathrm{C}_{14} \mathrm{H}_{21} \mathrm{~N}_{2} \mathrm{O}_{4} \mathrm{~S}^{+}, 313.1217$; found, 313.1218.

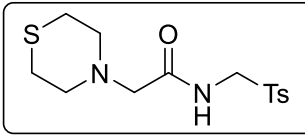

2-Thiomorpholino- $N$-(tosylmethyl)acetamide $\quad(5 \mathrm{~m}) . \quad$ Purified by analytical TLC on silica gel with petroleum/ethyl acetate $=1: 2$ as an eluent with $\mathrm{R}_{\mathrm{f}}=0.3$, white solid $(28.2 \mathrm{mg}, 43 \%), \mathrm{mp}=125-126^{\circ} \mathrm{C} .{ }^{1} \mathrm{H}$ NMR $(600$ $\left.\mathrm{MHz}, \mathrm{CDCl}_{3}\right) \delta 7.91(\mathrm{t}, J=6.6 \mathrm{~Hz}, 1 \mathrm{H}), 7.77(\mathrm{~d}, J=8.2 \mathrm{~Hz}, 2 \mathrm{H}), 7.35$ (d, $\left.J=7.9 \mathrm{~Hz}, 2 \mathrm{H}\right), 4.72(\mathrm{~d}$, $J=7.0 \mathrm{~Hz}, 2 \mathrm{H}), 2.93(\mathrm{~s}, 2 \mathrm{H}), 2.71-2.69(\mathrm{~m}, 8 \mathrm{H}), 2.45(\mathrm{~s}, 3 \mathrm{H}) .{ }^{13} \mathrm{C}\left\{{ }^{1} \mathrm{H}\right\} \mathrm{NMR}\left(151 \mathrm{MHz}, \mathrm{CDCl}_{3}\right)$ $\delta 169.9,145.5,134.1,129.9,128.6,61.8,59.8,55.2,27.9,21.7$. HRMS (ESI) $m / z:(\mathrm{M}+\mathrm{H})^{+}$calcd for $\mathrm{C}_{14} \mathrm{H}_{21} \mathrm{~N}_{2} \mathrm{O}_{3} \mathrm{~S}_{2}{ }^{+}, 329.0988$; found, 329.0987 .

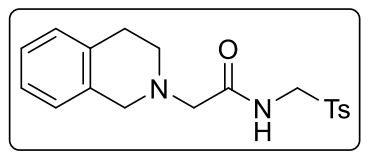

2-(3,4-Dihydroisoquinolin-2(1H)-yl)- $N$-(tosylmethyl)acetamide (5n). Purified by analytical TLC on silica gel with petroleum/ethyl acetate $=$ $1: 2$ as an eluent with $\mathrm{R}_{\mathrm{f}}=0.4$, white solid $(31.5 \mathrm{mg}, 44 \%), \mathrm{mp}=119-120$ ${ }^{\circ} \mathrm{C} .{ }^{1} \mathrm{H}$ NMR $\left(600 \mathrm{MHz}, \mathrm{CDCl}_{3}\right) \delta 8.02(\mathrm{t}, J=6.3 \mathrm{~Hz}, 1 \mathrm{H}), 7.76(\mathrm{~d}, J=8.2 \mathrm{~Hz}, 2 \mathrm{H}), 7.31$ (d, $J=8.1$ $\mathrm{Hz}, 2 \mathrm{H}), 7.20-7.13(\mathrm{~m}, 3 \mathrm{H}), 7.02(\mathrm{~d}, J=7.0 \mathrm{~Hz}, 1 \mathrm{H}), 4.70$ (d, $J=7.0 \mathrm{~Hz}, 2 \mathrm{H}), 3.70$ (s, 2H), 3.11 (s, 2H), $2.95(\mathrm{t}, J=5.8 \mathrm{~Hz}, 2 \mathrm{H}), 2.78(\mathrm{t}, J=5.9 \mathrm{~Hz}, 2 \mathrm{H}), 2.43(\mathrm{~s}, 3 \mathrm{H}) .{ }^{13} \mathrm{C}\left\{{ }^{1} \mathrm{H}\right\} \mathrm{NMR}(151 \mathrm{MHz}$, $\left.\mathrm{CDCl}_{3}\right) \delta 170.1,145.4,134.1,133.6,133.4,130.0,128.8,128.7,126.7,126.4,126.0,60.8,59.9$, 56.0, 51.5, 29.1, 21.7. HRMS (ESI) $m / z$ : $(\mathrm{M}+\mathrm{H})^{+}$calcd for $\mathrm{C}_{19} \mathrm{H}_{23} \mathrm{~N}_{2} \mathrm{O}_{3} \mathrm{~S}^{+}$, 359.1424; found, 359.1420 . 


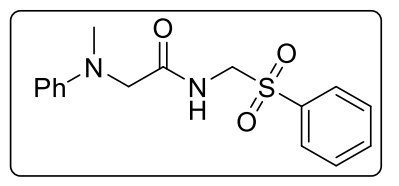

2-(Methyl(phenyl)amino)- $N$-((phenylsulfonyl)methyl)acetamide (6a). Purified by analytical TLC on silica gel with dichloromethane/ethyl acetate $=10: 1$ as an eluent with $\mathrm{R}_{\mathrm{f}}=0.3$, white solid (40.1 mg, 63\%), mp = 115-116 ${ }^{\circ} \mathrm{C} .{ }^{1} \mathrm{H} \mathrm{NMR}\left(600 \mathrm{MHz}, \mathrm{CDCl}_{3}\right)$ $\delta 7.84(\mathrm{~d}, J=7.9 \mathrm{~Hz}, 2 \mathrm{H}), 7.68(\mathrm{t}, J=7.5 \mathrm{~Hz}, 1 \mathrm{H}), 7.55(\mathrm{t}, J=7.6 \mathrm{~Hz}, 2 \mathrm{H}), 7.36(\mathrm{t}, J=6.4 \mathrm{~Hz}, 1 \mathrm{H})$, $7.28(\mathrm{t}, J=7.7 \mathrm{~Hz}, 2 \mathrm{H}), 6.88(\mathrm{t}, J=7.2 \mathrm{~Hz}, 1 \mathrm{H}), 6.67(\mathrm{~d}, J=8.1 \mathrm{~Hz}, 2 \mathrm{H}), 4.70(\mathrm{~d}, J=6.9 \mathrm{~Hz}, 2 \mathrm{H})$, $3.74(\mathrm{~s}, 2 \mathrm{H}), 2.98(\mathrm{~s}, 3 \mathrm{H}) .{ }^{13} \mathrm{C}\left\{{ }^{1} \mathrm{H}\right\} \mathrm{NMR}\left(151 \mathrm{MHz}, \mathrm{CDCl}_{3}\right) \delta 170.3,149.0,136.8,134.4,129.5$, 129.3, 128.8, 119.2, 113.4, 59.9, 58.5, 40.0. HRMS (ESI) $m / z:(\mathrm{M}+\mathrm{H})^{+}$calcd for $\mathrm{C}_{16} \mathrm{H}_{19} \mathrm{~N}_{2} \mathrm{O}_{3} \mathrm{~S}^{+}$, 319.1111; found, 319.1112 .

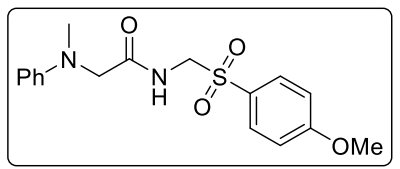

$N$-(((4-methoxyphenyl)sulfonyl)methyl)-2(methyl(phenyl)amino)acetamide (6b). Purified by analytical TLC on silica gel with dichloromethane/ethyl acetate $=10: 1$ as an eluent with $\mathrm{R}_{\mathrm{f}}=0.3$, white solid (45.3 $\left.\mathrm{mg}, 65 \%\right), \mathrm{mp}=112-113{ }^{\circ} \mathrm{C} .{ }^{1} \mathrm{H}$ NMR $\left(600 \mathrm{MHz}, \mathrm{CDCl}_{3}\right) \delta 7.75(\mathrm{~d}, J=8.8 \mathrm{~Hz}, 2 \mathrm{H}), 7.35(\mathrm{t}, J=6.4 \mathrm{~Hz}, 1 \mathrm{H}), 7.28(\mathrm{t}, J=7.9 \mathrm{~Hz}, 2 \mathrm{H}), 6.98$ $(\mathrm{d}, J=8.8 \mathrm{~Hz}, 2 \mathrm{H}), 6.88(\mathrm{t}, J=7.3 \mathrm{~Hz}, 1 \mathrm{H}), 6.67(\mathrm{~d}, J=8.3 \mathrm{~Hz}, 2 \mathrm{H}), 4.68(\mathrm{~d}, J=6.9 \mathrm{~Hz}, 2 \mathrm{H}), 3.88$ (s, 3H), $3.76(\mathrm{~s}, 2 \mathrm{H}), 2.98(\mathrm{~s}, 3 \mathrm{H}) .{ }^{13} \mathrm{C}\left\{{ }^{1} \mathrm{H}\right\} \mathrm{NMR}\left(151 \mathrm{MHz}, \mathrm{CDCl}_{3}\right) \delta 170.4,164.3,149.0,131.0$, 129.4, 128.0, 119.2, 114.5, 113.4, 60.0, 58.4, 55.7, 40.0. HRMS (ESI) $m / z:(\mathrm{M}+\mathrm{H})^{+}$calcd for $\mathrm{C}_{17} \mathrm{H}_{21} \mathrm{~N}_{2} \mathrm{O}_{4} \mathrm{~S}^{+}, 349.1217$; found, 349.1219.

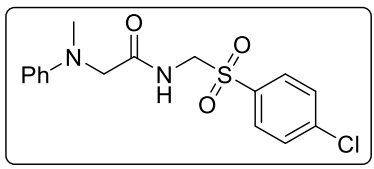

$\mathrm{N}$-(((4-chlorophenyl)sulfonyl)methyl)-2(methyl(phenyl)amino)acetamide (6c). Purified by analytical TLC on silica gel with dichloromethane/ethyl acetate $=10: 1$ as an eluent with $\mathrm{R}_{\mathrm{f}}=0.4$, white solid $(48.7 \mathrm{mg}, 69 \%), \mathrm{mp}=134-135^{\circ} \mathrm{C} .{ }^{1} \mathrm{H}$ NMR $(600$

$\left.\mathrm{MHz}, \mathrm{CDCl}_{3}\right) \delta 7.75(\mathrm{~d}, J=8.5 \mathrm{~Hz}, 2 \mathrm{H}), 7.50(\mathrm{~d} J=8.5 \mathrm{~Hz}, 2 \mathrm{H}), 7.36(\mathrm{t}, J=6.5 \mathrm{~Hz}, 1 \mathrm{H}), 7.29(\mathrm{t}, J$ $=8.0 \mathrm{~Hz}, 2 \mathrm{H}), 6.90(\mathrm{t}, J=7.3 \mathrm{~Hz}, 1 \mathrm{H}), 6.66(\mathrm{~d}, J=8.3 \mathrm{~Hz}, 2 \mathrm{H}), 4.68(\mathrm{~d}, J=6.9 \mathrm{~Hz}, 2 \mathrm{H}), 3.76(\mathrm{~s}$, 2H), 2.99 (s, 3H). ${ }^{13} \mathrm{C}\left\{{ }^{1} \mathrm{H}\right\}$ NMR (151 MHz, $\left.\mathrm{CDCl}_{3}\right) \delta 170.4,148.9,141.3,135.4,130.3,129.6$, 129.5, 119.4, 113.5, 60.0, 58.4, 40.2. HRMS (ESI) $m / z$ : $(\mathrm{M}+\mathrm{Na})^{+}$calcd for $\mathrm{C}_{16} \mathrm{H}_{17} \mathrm{ClN}_{2} \mathrm{O}_{3} \mathrm{SNa}^{+}$, 375.0540; found, 375.0539 .

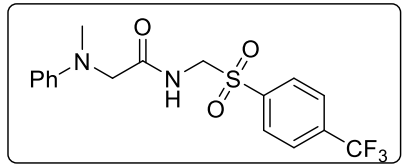

2-(Methyl(phenyl)amino)- $N$-(((4(trifluoromethyl)phenyl)sulfonyl)methyl)acetamide (6d). Purified by analytical TLC on silica gel with dichloromethane/ethyl acetate $=$ 10:1 as an eluent with $\mathrm{R}_{\mathrm{f}}=0.4$, white solid $(40.2 \mathrm{mg}, 52 \%), \mathrm{mp}=$ 104-105 ${ }^{\circ} \mathrm{C} .{ }^{1} \mathrm{H}$ NMR $\left(600 \mathrm{MHz}, \mathrm{CDCl}_{3}\right) \delta 7.96(\mathrm{~d}, J=8.2 \mathrm{~Hz}, 2 \mathrm{H}), 7.79(\mathrm{~d}, J=8.3 \mathrm{~Hz}, 2 \mathrm{H}), 7.35$ (t, $J=6.4 \mathrm{~Hz}, 1 \mathrm{H}), 7.31-7.28(\mathrm{~m}, 2 \mathrm{H}), 6.91(\mathrm{t}, J=7.3 \mathrm{~Hz}, 1 \mathrm{H}), 6.67(\mathrm{~d}, J=8.1 \mathrm{~Hz}, 2 \mathrm{H}), 4.75(\mathrm{~d}$, $J=6.9 \mathrm{~Hz}, 2 \mathrm{H}), 3.77(\mathrm{~s}, 2 \mathrm{H}), 3.00(\mathrm{~s}, 3 \mathrm{H}) .{ }^{13} \mathrm{C}\left\{{ }^{1} \mathrm{H}\right\}\left(151 \mathrm{MHz}, \mathrm{CDCl}_{3}\right) \delta 170.5,148.8,140.6,136.0$ $(\mathrm{q}, J=33.4 \mathrm{~Hz}), 129.6,129.4,126.4(\mathrm{q}, J=3.5 \mathrm{~Hz}), 123.03$ (q, $J=273.4 \mathrm{~Hz}), 119.5,113.5,60.0$, 58.4, 40.3. ${ }^{19} \mathrm{~F}$ NMR $\left(565 \mathrm{MHz}, \mathrm{CDCl}_{3}\right) \delta$-63.22. HRMS (ESI) $m / z:(\mathrm{M}+\mathrm{H})^{+}$calcd for $\mathrm{C}_{17} \mathrm{H}_{18} \mathrm{~F}_{3} \mathrm{~N}_{2} \mathrm{O}_{3} \mathrm{~S}^{+}, 387.0985$; found, 387.0988 .

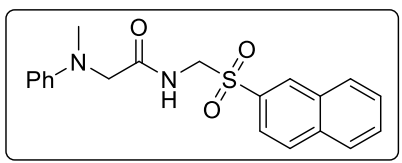

2-(Methyl(phenyl)amino)- $N$-((naphthalen-2ylsulfonyl)methyl)acetamide (6e). Purified by analytical TLC on silica gel with dichloromethane/ethyl acetate $=10: 1$ as an eluent with $\mathrm{R}_{\mathrm{f}}=0.4$, white solid $(32.4 \mathrm{mg}, 44 \%), \mathrm{mp}=116-117^{\circ} \mathrm{C} .{ }^{1} \mathrm{H}$ NMR $(600$ 
MHz, $\left.\mathrm{CDCl}_{3}\right) \delta 8.42(\mathrm{~s}, 1 \mathrm{H}), 7.98(\mathrm{~d}, \mathrm{~J}=8.6 \mathrm{~Hz}, 1 \mathrm{H}), 7.94(\mathrm{~d}, \mathrm{~J}=9.2 \mathrm{~Hz}, 2 \mathrm{H}), 7.81$ (dd, $J=8.6$, $1.5 \mathrm{~Hz}, 1 \mathrm{H}), 7.69$ (t, $J=7.5 \mathrm{~Hz}, 1 \mathrm{H}), 7.64(\mathrm{t}, J=7.5 \mathrm{~Hz}, 1 \mathrm{H}), 7.43(\mathrm{t}, J=4.3 \mathrm{~Hz}, 1 \mathrm{H}), 7.24$ (t, $J=$ $7.5 \mathrm{~Hz}, 2 \mathrm{H}), 6.87(\mathrm{t}, J=7.3 \mathrm{~Hz}, 1 \mathrm{H}), 6.63(\mathrm{~d}, J=8.4 \mathrm{~Hz}, 2 \mathrm{H}), 4.78(\mathrm{~d}, J=6.9 \mathrm{~Hz}, 2 \mathrm{H}), 3.68(\mathrm{~s}, 2 \mathrm{H})$, $2.94(\mathrm{~s}, 3 \mathrm{H}) .{ }^{13} \mathrm{C}\left\{{ }^{1} \mathrm{H}\right\} \mathrm{NMR}(151 \mathrm{MHz}, \mathrm{CDCl} 3) \delta 170.4,149.0,135.6,133.7,132.0,131.0,129.7$, 129.6, 129.5, 129.4, 128.1, 127.8, 123.0, 119.2, 113.4, 59.9, 58.5, 40.0. HRMS (ESI) $\mathrm{m} / \mathrm{z}:(\mathrm{M}+\mathrm{H})^{+}$ calcd for $\mathrm{C}_{20} \mathrm{H}_{21} \mathrm{~N}_{2} \mathrm{O}_{3} \mathrm{~S}^{+}, 369.1268$; found, 369.1271 .

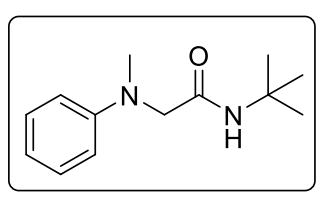

$\mathrm{N}$-(tert-butyl)-2-(methyl(phenyl)amino)acetamide (6f). Purified by analytical TLC on silica gel with petroleum/ethyl acetate $=2: 1$ as an eluent with $\mathrm{R}_{\mathrm{f}}=0.4$, white solid $(18.5 \mathrm{mg}, 42 \%)$. The spectroscopic data correspond to those previously reported in the literature. ${ }^{3}{ }^{1} \mathrm{H}$ NMR $(600$ MHz, $\left.\mathrm{CDCl}_{3}\right) \delta 7.27(\mathrm{t}, J=7.3 \mathrm{~Hz}, 2 \mathrm{H}), 6.85(\mathrm{t}, J=7.1 \mathrm{~Hz}, 1 \mathrm{H}), 6.74(\mathrm{~d}, J=7.7 \mathrm{~Hz}, 2 \mathrm{H}), 6.39(\mathrm{br}$, $1 \mathrm{H}), 3.72(\mathrm{~s}, 2 \mathrm{H}), 2.98(\mathrm{~s}, 3 \mathrm{H}), 1.33(\mathrm{~s}, 9 \mathrm{H}) .{ }^{13} \mathrm{C}\left\{{ }^{1} \mathrm{H}\right\} \mathrm{NMR}(151 \mathrm{MHz}, \mathrm{CDCl} 3) \delta 169.5,149.5$, 129.3, 118.7, 113.4, 59.9, 51.0, 39.8, 28.7. HRMS (ESI) $m / z:(\mathrm{M}+\mathrm{H})^{+}$calcd for $\mathrm{C}_{13} \mathrm{H}_{21} \mathrm{~N}_{2} \mathrm{O}^{+}$, 221.1649; found, 221.1643.

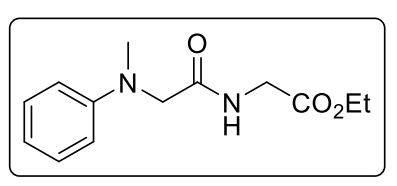

Ethyl $N$-methyl- $N$-phenylglycylglycinate (6g). Purified by analytical TLC on silica gel with petroleum/ethyl acetate $=1: 1$ as an eluent with $\mathrm{R}_{\mathrm{f}}=0.4$, white solid $(17.5 \mathrm{mg}, 35 \%)$. The spectroscopic data correspond to those previously reported in the literature. ${ }^{3}{ }^{1} \mathrm{H}$ NMR $\left(600 \mathrm{MHz}, \mathrm{CDCl}_{3}\right) \delta 7.27(\mathrm{t}, J=7.4 \mathrm{~Hz}, 2 \mathrm{H}), 7.06(\mathrm{br}, 1 \mathrm{H}), 6.84(\mathrm{t}, J=7.2 \mathrm{~Hz}, 1 \mathrm{H}), 6.77(\mathrm{~d}, J=7.9$ $\mathrm{Hz}, 2 \mathrm{H}), 4.18(\mathrm{q}, J=7.0 \mathrm{~Hz}, 2 \mathrm{H}), 4.05(\mathrm{~d}, J=5.4 \mathrm{~Hz}, 2 \mathrm{H}), 3.90(\mathrm{~s}, 2 \mathrm{H}), 3.04$ (s, 3H), $1.26(\mathrm{t}, J=$ $7.0 \mathrm{~Hz}, 3 \mathrm{H}) .{ }^{13} \mathrm{C}\left\{{ }^{1} \mathrm{H}\right\} \mathrm{NMR}(151 \mathrm{MHz}, \mathrm{CDCl} 3) \delta 171.0,169.5,149.4,129.4,118.84,113.4,61.5$, 58.8, 41.0, 39.7, 14.1. HRMS (ESI) $m / z$ : $(\mathrm{M}+\mathrm{H})^{+}$calcd for $\mathrm{C}_{13} \mathrm{H}_{19} \mathrm{~N}_{2} \mathrm{O}_{3}{ }^{+}, 251.1390$; found, 251.1385 .

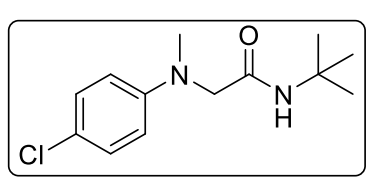

$\mathrm{N}$-(tert-butyl)-2-((4-chlorophenyl)(methyl)amino)acetamide (6h). Purified by analytical TLC on silica gel with petroleum/ethyl acetate $=$ $2: 1$ as an eluent with $R_{f}=0.3$, white solid ( $20.4 \mathrm{mg}, 40 \%$ ). The spectroscopic data correspond to those previously reported in the literature. ${ }^{6}{ }^{1} \mathrm{H} \mathrm{NMR}\left(600 \mathrm{MHz}, \mathrm{CDCl}_{3}\right) \delta{ }^{1} \mathrm{H} \mathrm{NMR}\left(600 \mathrm{MHz}, \mathrm{CDCl}_{3}\right) \delta 7.21(\mathrm{~d}, J=7.7 \mathrm{~Hz}, 2 \mathrm{H})$, $6.64(\mathrm{~d}, J=7.7 \mathrm{~Hz}, 2 \mathrm{H}), 6.24$ (br, $1 \mathrm{H}), 3.70(\mathrm{~s}, 2 \mathrm{H}), 2.98$ (s, 3H), 1.32 (s, 9H). ${ }^{13} \mathrm{C}\left\{{ }^{1} \mathrm{H}\right\} \mathrm{NMR}(151$ $\mathrm{MHz}, \mathrm{CDCl} 3) \delta 169.0,148.0,129.1,123.7,114.4,59.7,51.0,40.1,28.7$. HRMS (ESI) $m / z:(\mathrm{M}+$ $\mathrm{H})^{+}$calcd for $\mathrm{C}_{13} \mathrm{H}_{20} \mathrm{ClN}_{2} \mathrm{O}^{+}, 255.1259$; found, 255.1254.

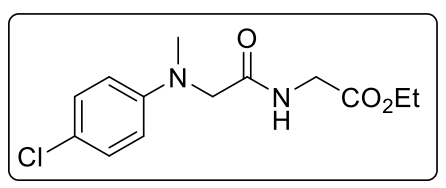

Ethyl $\quad N$-(4-chlorophenyl)- $N$-methylglycylglycinate (6i). Purified by analytical TLC on silica gel with petroleum/ethyl acetate $=1: 1$ as an eluent with $\mathrm{R}_{\mathrm{f}}=0.3$, white solid $(18.2 \mathrm{mg}, 32 \%)$. The spectroscopic data correspond to those previously reported in the literature. ${ }^{3}{ }^{1} \mathrm{H}$ NMR (600 MHz, $\left.\mathrm{CDCl}_{3}\right) \delta{ }^{1} \mathrm{H}$ NMR $\left(600 \mathrm{MHz}, \mathrm{CDCl}_{3}\right) \delta 7.21(\mathrm{~d}, J=7.8 \mathrm{~Hz}$, 2H), $6.96(\mathrm{br}, 1 \mathrm{H}), 6.68$ (d, $J=7.9 \mathrm{~Hz}, 2 \mathrm{H}), 4.19$ (q, $J=7.0 \mathrm{~Hz}, 2 \mathrm{H}), 4.05$ (d, $J=5.2 \mathrm{~Hz}, 2 \mathrm{H}), 3.88$ (s, 2H), $3.03(\mathrm{~s}, 3 \mathrm{H}), 1.26(\mathrm{t}, J=6.9 \mathrm{~Hz}, 3 \mathrm{H}) .{ }^{13} \mathrm{C}\left\{{ }^{1} \mathrm{H}\right\} \mathrm{NMR}(151 \mathrm{MHz}, \mathrm{CDCl} 3) \delta 170.5,169.5$, $147.9,129.2,123.8,114.5,61.6,58.7,41.0,39.9,14.1$. HRMS (ESI) $m / z:(\mathrm{M}+\mathrm{H})^{+}$calcd for $\mathrm{C}_{13} \mathrm{H}_{18} \mathrm{ClN}_{2} \mathrm{O}_{3}^{+}$, 285.1001; found, 285.0998. 


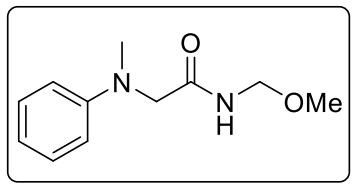

$N$-(methoxymethyl)-2-(methyl(phenyl)amino)acetamide (7). Purified by preparative TLC on silica gel plates using petroleum/ethyl acetate $=$ $1 / 1$ as the eluent to give the desired product 7 in $87 \%$ yield. $R_{f}=0.5$, yellow oil (36.2 mg, 87\%). ${ }^{1} \mathrm{H} \mathrm{NMR}\left(600 \mathrm{MHz}, \mathrm{CDCl}_{3}\right) \delta 7.29-7.26(\mathrm{~m}$, $3 \mathrm{H}), 6.85$ (t, $J=7.3 \mathrm{~Hz}, 1 \mathrm{H}), 6.74(\mathrm{~d}, J=8.2 \mathrm{~Hz}, 2 \mathrm{H}), 4.70(\mathrm{~d}, J=7.0 \mathrm{~Hz}, 2 \mathrm{H}), 3.90(\mathrm{~s}, 2 \mathrm{H}), 3.30$ (s, 3H), $3.03(\mathrm{~s}, 3 \mathrm{H}) .{ }^{13} \mathrm{C}\left\{{ }^{1} \mathrm{H}\right\} \mathrm{NMR}\left(151 \mathrm{MHz}, \mathrm{CDCl}_{3}\right) \delta 171.6,149.1,129.4,118.9,113.2,71.1$, 58.8, 56.0, 39.9. HRMS (ESI) $m / z$ : $(\mathrm{M}+\mathrm{H})^{+}$calcd for $\mathrm{C}_{11} \mathrm{H}_{17} \mathrm{~N}_{2} \mathrm{O}_{2}{ }^{+}, 209.1285$; found, 209.1284.

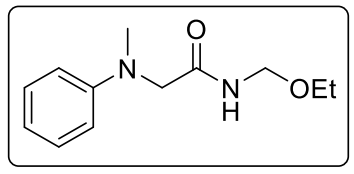

$N$-(ethoxymethyl)-2-(methyl(phenyl)amino)acetamide (8). Purified by preparative TLC on silica gel plates using petroleum/ethyl acetate $=$ $1 / 1$ as the eluent to give the desired product $\mathbf{8}$ in $86 \%$ yield. $\mathrm{R}_{\mathrm{f}}=0.7$, yellow oil (38.2 mg, 86\%). ${ }^{1} \mathrm{H}$ NMR $\left(600 \mathrm{MHz}, \mathrm{CDCl}_{3}\right) \delta 7.28-7.23(\mathrm{~m}$, $3 \mathrm{H}), 6.85(\mathrm{t}, J=7.3 \mathrm{~Hz}, 1 \mathrm{H}), 6.74(\mathrm{~d}, J=8.1 \mathrm{~Hz}, 2 \mathrm{H}), 4.74(\mathrm{~d}, J=6.9 \mathrm{~Hz}, 2 \mathrm{H}), 3.89$ (s, 2H), 3.51 (q, $J=7.0 \mathrm{~Hz}, 2 \mathrm{H}), 3.03(\mathrm{~s}, 3 \mathrm{H}), 1.16(\mathrm{t}, J=7.0 \mathrm{~Hz}, 3 \mathrm{H}) .{ }^{13} \mathrm{C}\left\{{ }^{1} \mathrm{H}\right\} \mathrm{NMR}\left(151 \mathrm{MHz}, \mathrm{CDCl}_{3}\right) \delta 171.4$, 149.1, 129.4, 118.8, 113.2, 69.6, 64.0, 58.8, 40.0, 15.0. HRMS (ESI) $m / z:(\mathrm{M}+\mathrm{H})^{+}$calcd for $\mathrm{C}_{12} \mathrm{H}_{19} \mathrm{~N}_{2} \mathrm{O}_{2}{ }^{+}, 223.1441$; found, 223.1437.

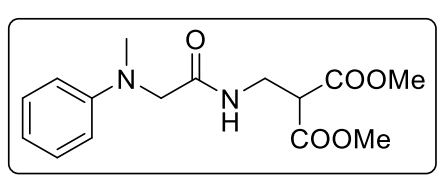

\section{Dimethyl}

2-((2(methyl(phenyl)amino)acetamido)methyl)malonate Purified by preparative TLC on silica gel plates using petroleum/ethyl acetate $=1 / 1$ as the eluent to give the desired product 9 in $93 \%$ yield. $\mathrm{R}_{\mathrm{f}}=0.3$, yellow oil $(57.4 \mathrm{mg}, 93 \%)$. ${ }^{1} \mathrm{H}$ NMR $\left(600 \mathrm{MHz}, \mathrm{CDCl}_{3}\right) \delta 7.26-$ $7.24(\mathrm{~m}, 2 \mathrm{H}), 7.06(\mathrm{t}, J=6.1 \mathrm{~Hz}, 1 \mathrm{H}), 6.82(\mathrm{t}, J=7.3 \mathrm{~Hz}, 1 \mathrm{H}), 6.70(\mathrm{~d}, J=8.1 \mathrm{~Hz}, 2 \mathrm{H}), 3.83(\mathrm{~s}$, 2H), $3.76(\mathrm{t}, J=6.5 \mathrm{~Hz}, 2 \mathrm{H}), 3.69(\mathrm{t}, J=6.5 \mathrm{~Hz}, 1 \mathrm{H}), 3.66(\mathrm{~s}, 6 \mathrm{H}), 3.00(\mathrm{~s}, 3 \mathrm{H}) .{ }^{13} \mathrm{C}\left\{{ }^{1} \mathrm{H}\right\} \mathrm{NMR}(151$ $\left.\mathrm{MHz}, \mathrm{CDCl}_{3}\right) \delta 170.8,168.2,149.1,129.3,118.6,113.1,58.6,52.7,50.9,39.7,37.6$. HRMS (ESI) $m / z:(\mathrm{M}+\mathrm{H})^{+}$calcd for $\mathrm{C}_{15} \mathrm{H}_{21} \mathrm{~N}_{2} \mathrm{O}_{5}{ }^{+}, 309.1445$; found, 309.1446.

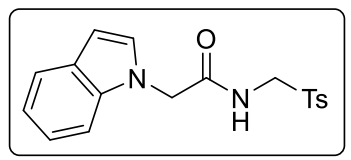

2-(1H-indol-1-yl)- $N$-(tosylmethyl)acetamide (10). Purified by preparative TLC on silica gel plates using dichloromethane/ethyl acetate $=10: 1$ as the eluent to give the desired product 10 in $80 \%$ yield. $R_{\mathrm{f}}=0.3$, white solid (54.8 mg, 80\%), mp $=139-140{ }^{\circ} \mathrm{C} .{ }^{1} \mathrm{H}$ NMR $(600 \mathrm{MHz}$, DMSO) $\delta 9.25(\mathrm{t}, J=6.6 \mathrm{~Hz}, 1 \mathrm{H}), 7.66(\mathrm{~d}, J=8.2 \mathrm{~Hz}, 2 \mathrm{H}), 7.53(\mathrm{~d}, J=7.8 \mathrm{~Hz}, 1 \mathrm{H}), 7.30(\mathrm{~d}, J=$ $8.0 \mathrm{~Hz}, 2 \mathrm{H}), 7.19(\mathrm{~d}, J=3.1 \mathrm{~Hz}, 1 \mathrm{H}), 7.16(\mathrm{~d}, J=8.2 \mathrm{~Hz}, 1 \mathrm{H}), 7.11(\mathrm{t}, J=7.5 \mathrm{~Hz}, 1 \mathrm{H}), 7.03(\mathrm{t}, J=$ $7.4 \mathrm{~Hz}, 1 \mathrm{H}), 6.40(\mathrm{~d}, J=3.1 \mathrm{~Hz}, 1 \mathrm{H}), 4.81(\mathrm{~s}, 2 \mathrm{H}), 4.71(\mathrm{~d}, J=6.6 \mathrm{~Hz}, 2 \mathrm{H}), 2.35(\mathrm{~s}, 3 \mathrm{H}) .{ }^{13} \mathrm{C}\left\{{ }^{1} \mathrm{H}\right\}$ NMR (151 MHz, DMSO) $\delta 168.1,145.0,136.6,134.7,130.2,130.1,128.9,128.5,121.5,120.7$, 119.6, 110.2, 101.3, 60.5, 48.6, 21.6. HRMS (ESI) $m / z$ : $(\mathrm{M}+\mathrm{H})^{+}$calcd for $\mathrm{C}_{18} \mathrm{H}_{19} \mathrm{~N}_{2} \mathrm{O}_{3} \mathrm{~S}^{+}, 343.111$; found, 343.1112 .

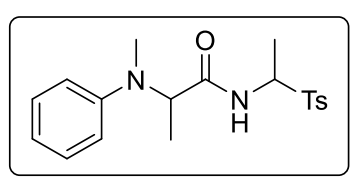

\section{2-(Methyl(phenyl)amino)- $N$-(1-tosylethyl)propanamide}

(11a).

Purified by analytical TLC on silica gel with dichloromethane as an eluent with $\mathrm{R}_{\mathrm{f}}=0.4$, yellow oil $(37.5 \mathrm{mg}, 52 \%)$. Ratio of rotamers: $1.5: 1$. Major product: ${ }^{1} \mathrm{H} \mathrm{NMR}\left(600 \mathrm{MHz}, \mathrm{CDCl}_{3}\right) \delta 7.79(\mathrm{~d}, J=8.2 \mathrm{~Hz}, 2 \mathrm{H})$, $7.35(\mathrm{~d}, J=7.8 \mathrm{~Hz}, 2 \mathrm{H}), 7.31-7.26(\mathrm{~m}, 2 \mathrm{H}), 7.14(\mathrm{t}, J=12.1 \mathrm{~Hz}, 1 \mathrm{H}), 6.88(\mathrm{t}, J=7.1 \mathrm{~Hz}, 1 \mathrm{H})$, $6.81(\mathrm{~d}, J=8.2 \mathrm{~Hz}, 2 \mathrm{H}), 5.43-5.38(\mathrm{~m}, 1 \mathrm{H}), 4.22(\mathrm{q}, J=7.0 \mathrm{~Hz}, 1 \mathrm{H}), 2.72(\mathrm{~s}, 3 \mathrm{H}), 2.45(\mathrm{~s}, 3 \mathrm{H})$, $1.50(\mathrm{~d}, J=7.0 \mathrm{~Hz}, 3 \mathrm{H}), 1.18(\mathrm{~d}, J=7.0 \mathrm{~Hz}, 3 \mathrm{H}) .{ }^{13} \mathrm{C}\left\{{ }^{1} \mathrm{H}\right\}\left(151 \mathrm{MHz}, \mathrm{CDCl}_{3}\right) \delta 172.8,149.6,145.3$, 
133.6, 129.8, 129.4, 129.1, 119.5, 114.9, 64.8, 60.4, 33.9, 21.7, 13.9, 12.4. Minor product: ${ }^{1} \mathrm{H}$ NMR $\left(600 \mathrm{MHz}, \mathrm{CDCl}_{3}\right) \delta 7.75(\mathrm{~d}, J=8.2 \mathrm{~Hz}, 2 \mathrm{H}), 7.34(\mathrm{~d}, J=7.8 \mathrm{~Hz}, 2 \mathrm{H}), 7.31-7.26(\mathrm{~m}, 2 \mathrm{H}), 7.14$ $(\mathrm{t}, J=12.1 \mathrm{~Hz}, 1 \mathrm{H}), 6.90(\mathrm{t}, J=7.1 \mathrm{~Hz}, 1 \mathrm{H}), 6.77(\mathrm{~d}, J=8.2 \mathrm{~Hz}, 2 \mathrm{H}), 5.39-5.33(\mathrm{~m}, 1 \mathrm{H}), 4.01(\mathrm{q}$, $J=7.0 \mathrm{~Hz}, 1 \mathrm{H}), 2.72(\mathrm{~s}, 3 \mathrm{H}), 2.45(\mathrm{~s}, 3 \mathrm{H}), 1.56(\mathrm{~d}, J=7.0 \mathrm{~Hz}, 3 \mathrm{H}), 1.20(\mathrm{~d}, J=7.0 \mathrm{~Hz}, 3 \mathrm{H}) .{ }^{13} \mathrm{C}\left\{{ }^{1} \mathrm{H}\right\}$ $\left(151 \mathrm{MHz}, \mathrm{CDCl}_{3}\right) \delta 172.3,149.0,145.3,133.3,129.8,129.3,129.2,119.7,115.3,64.9,60.7,34.4$, 21.8, 13.6, 11.3. HRMS (ESI) $m / z$ : $(\mathrm{M}+\mathrm{H})^{+}$calcd for $\mathrm{C}_{19} \mathrm{H}_{25} \mathrm{~N}_{2} \mathrm{O}_{3} \mathrm{~S}^{+}, 361.1581$; found, 361.1576.

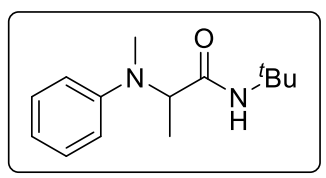

$N$-(tert-butyl)-2-(methyl(phenyl)amino)propanamide (11b). Purified by preparative TLC on silica gel with dichloromethane as the eluent with $\mathrm{R}_{\mathrm{f}}=$ 0.4 , yellow oil (10.8 mg, 23\%). ${ }^{1} \mathrm{H}$ NMR $\left(600 \mathrm{MHz}, \mathrm{CDCl}_{3}\right) \delta 7.29-7.26$ (m, 2H), $6.86-6.83(\mathrm{~m}, 2 \mathrm{H}), 6.48(\mathrm{br}, 1 \mathrm{H}), 4.23$ (q, $J=7.0 \mathrm{~Hz}, 1 \mathrm{H}), 2.77$ (s, 3H), $1.33-1.31(\mathrm{~m}, 12 \mathrm{H}) .{ }^{13} \mathrm{C}\left\{{ }^{1} \mathrm{H}\right\} \mathrm{NMR}\left(151 \mathrm{MHz}, \mathrm{CDCl}_{3}\right) \delta 172.1,149.6,129.3,118.9,114.8$, 60.8, 50.8, 34.0, 28.7, 11.6. HRMS (ESI) $m / z$ : $(\mathrm{M}+\mathrm{H})^{+}$calcd for $\mathrm{C}_{14} \mathrm{H}_{23} \mathrm{~N}_{2} \mathrm{O}^{+}, 235.1805$; found, 235.1803 .

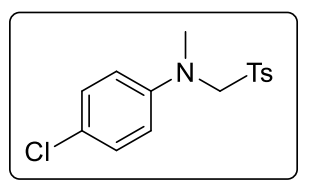

4-Chloro- $N$-methyl- $N$-(tosylmethyl)aniline (12). The solid was filtered and washed with $\mathrm{MeOH}$ to give product 12 in $95 \%$ yield. White solid (294.3 $\mathrm{mg}, 95 \%), \mathrm{mp}=130-131^{\circ} \mathrm{C} .{ }^{1} \mathrm{H}$ NMR $\left(600 \mathrm{MHz}, \mathrm{CDCl}_{3}\right) \delta 7.71(\mathrm{~d}, J=7.4$ $\mathrm{Hz}, 2 \mathrm{H}), 7.26(\mathrm{~d}, J=7.2 \mathrm{~Hz}, 2 \mathrm{H}), 7.05(\mathrm{~d}, J=7.7 \mathrm{~Hz}, 2 \mathrm{H}), 6.55(\mathrm{~d}, J=7.7$ $\mathrm{Hz}, 2 \mathrm{H}), 4.70$ (s, 2H), 2.97 (s, 3H), $2.41(\mathrm{~s}, 3 \mathrm{H}) .{ }^{13} \mathrm{C}\left\{{ }^{1} \mathrm{H}\right\}\left(151 \mathrm{MHz}, \mathrm{CDCl}_{3}\right) \delta$ 145.5, 145.3, 135.7, 130.0, 128.9, 128.8, 124.0, 114.3, 75.9, 39.5, 21.6. HRMS (ESI) $\mathrm{m} / z:(\mathrm{M}+\mathrm{H})^{+}$calcd for $\mathrm{C}_{15} \mathrm{H}_{17} \mathrm{ClNO}_{2} \mathrm{~S}^{+}, 310.0663$; found, 310.0664 .

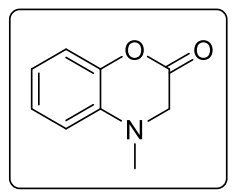

4-Methyl-3,4-dihydro-2 $H$-benzo $[b][1,4]$ oxazin-2-one (13). Purified by preparative TLC on silica gel with petroleum/ethyl acetate $=5 / 1$ as the eluent with $\mathrm{R}_{\mathrm{f}}=0.3$, yellow oil $(3.6 \mathrm{mg}, 11 \%) .{ }^{1} \mathrm{H} \mathrm{NMR}\left(600 \mathrm{MHz}, \mathrm{CDCl}_{3}\right) \delta 7.10(\mathrm{t}, J=7.7$ $\mathrm{Hz}, 1 \mathrm{H}), 7.06(\mathrm{~d}, J=7.9 \mathrm{~Hz}, 1 \mathrm{H}), 6.87(\mathrm{t}, J=7.6 \mathrm{~Hz}, 1 \mathrm{H}), 6.78(\mathrm{~d}, J=7.9 \mathrm{~Hz}$, 1H), 3.80 (s, 2H), 2.86 (s, 3H). ${ }^{13} \mathrm{C}\left\{{ }^{1} \mathrm{H}\right\}$ NMR (151 MHz, $\left.\mathrm{CDCl}_{3}\right) \delta 164.8,141.8,135.6,125.3$, 120.0, 116.8, 112.5, 52.6, 37.1. HRMS (ESI) $\mathrm{m} / z:(\mathrm{M}+\mathrm{H})^{+}$calcd for $\mathrm{C}_{9} \mathrm{H}_{10} \mathrm{NO}_{2}{ }^{+}, 164.0706$; found, 164.0710 .

(1) Han, C.; Fu, Z.; Guo, S.; Fang, X.; Lin, A.; Yao, H. Palladium-Catalyzed Remote 1,n-Arylamination of Unactivated Terminal Alkenes. ACS Catal. 2019, 9, 4196-4202.

(2) Lujan-Montelongo, J. A.; Estevez, A. O.; Fleming, F. F. Alkyl Sulfinates: Formal Nucleophiles for Synthesizing TosMIC Analogs. Eur. J. Org. Chem. 2015, 1602-1605.

(3) Guerrero, I.; Segundo, M. S.; Correa, A. Iron-catalyzed $\mathrm{C}\left(\mathrm{sp}^{3}\right)-\mathrm{H}$ functionalization of $N, N-$ dimethylanilines with isocyanides. Chem. Commun. 2018, 54, 1627.

(4) Ye, X.; Xie, C.; Huang, R.; Liu, J. Direct Synthesis of $\alpha$-Amino Amides from $N$-Alkyl Amines by the Copper-Catalyzed Oxidative Ugi-Type Reaction. Synlett 2012, 23, 409-412.

(5) Rueping, M.; Vila, C. Visible Light Photoredox Catalyzed Multi-Component Reactions. Org. Lett. 2013, 15, 2092-2095.

(6) Li, D.; Shen, X.; Lei, J. Metal-Free Iodine/TEMPO-Mediated Aerobic Oxidative Ugi-Type Multicomponent Reactions with Tertiary Amines. J. Org. Chem. 2020, 85, 2466-2475. 


\section{X-ray Crystal Structure of 3a, 5d, $5 \mathrm{~m}$, and $5 \mathrm{n}$}

Single crystals of complexes 3a (CCDC file Number 2109915), 5d (CCDC file Number 2109916), 5m (CCDC file Number 2109913), and $\mathbf{5 n}$ (CCDC file Number 2109914) were grown from DCM $/ n$ hexane, which were further confirmed by X-ray diffraction. Single-crystal X-ray diffraction data for the reported complex was recorded at 293(2) K on a Saturn CCD diffractometer, the data were collected on an Oxford Diffraction Gemini E diffractometer with graphite-monochromated $\mathrm{Cu} \mathrm{K \alpha}$ radiation $(\lambda=1.54184 \AA$ ) for compounds $3 \mathbf{3 a}, \mathbf{5 d}, \mathbf{5 m}$, and $\mathbf{5 n}$. The structures were solved by Direct Method of SHELXS-97 and refined by full-matrix least-squares techniques using the SHELXL-97 program. Non-hydrogen atoms were refined with anisotropic temperature parameters, and hydrogen atoms of the ligands were refined as rigid groups. Details of the crystal structure determination are summarized below. Further information can be found in the CIF file.

Table S7. Crystal data and structure refinement of 3a

\begin{tabular}{|c|c|}
\hline Structure & $3 \mathbf{a}$ \\
\hline Identification code & 202107216 \\
\hline Empirical formula & $\mathrm{C}_{17} \mathrm{H}_{20} \mathrm{~N}_{2} \mathrm{O}_{3} \mathrm{~S}$ \\
\hline Formula weight & 332.41 \\
\hline Temperature/K & $293(2)$ \\
\hline Crystal system & monoclinic \\
\hline Space group & $\mathrm{P} 2{ }_{1} / \mathrm{c}$ \\
\hline $\mathrm{a} / \AA$ & $20.1630(8)$ \\
\hline $\mathrm{b} / \AA$ & $5.13320(10)$ \\
\hline $\mathrm{c} / \AA$ & $16.8538(5)$ \\
\hline$\alpha /{ }^{\circ}$ & 90 \\
\hline$\beta /{ }^{\circ}$ & $109.190(4)$ \\
\hline$\gamma /{ }^{\circ}$ & 90 \\
\hline Volume $/ \AA^{3}$ & $1647.45(10)$ \\
\hline $\mathrm{Z}$ & 4 \\
\hline$\rho_{\text {calc }} \mathrm{g} / \mathrm{cm}^{3}$ & 1.340 \\
\hline$\mu / \mathrm{mm}^{-1}$ & 1.886 \\
\hline $\mathrm{F}(000)$ & 704.0 \\
\hline Crystal size $/ \mathrm{mm}^{3}$ & $0.22 \times 0.13 \times 0.09$ \\
\hline Radiation & $\operatorname{CuK} \alpha(\lambda=1.54184)$ \\
\hline $2 \Theta$ range for data collection $/{ }^{\circ}$ & 9.288 to 134.076 \\
\hline Index ranges & $-24 \leq \mathrm{h} \leq 24,-3 \leq \mathrm{k} \leq 6,-20 \leq 1 \leq 20$ \\
\hline Reflections collected & 6027 \\
\hline Independent reflections & $2927\left[R_{\text {int }}=0.0303, R_{\text {sigma }}=0.0395\right]$ \\
\hline Data/restraints/parameters & $2927 / 0 / 214$ \\
\hline Goodness-of-fit on $\mathrm{F}^{2}$ & 1.038 \\
\hline Final $\mathrm{R}$ indexes $[\mathrm{I}>=2 \sigma(\mathrm{I})]$ & $\mathrm{R} 1=0.0437, \mathrm{wR}_{2}=0.1102$ \\
\hline Final $\mathrm{R}$ indexes [all data] & $\mathrm{R} 1=0.0561, \mathrm{wR} 2=0.1211$ \\
\hline
\end{tabular}




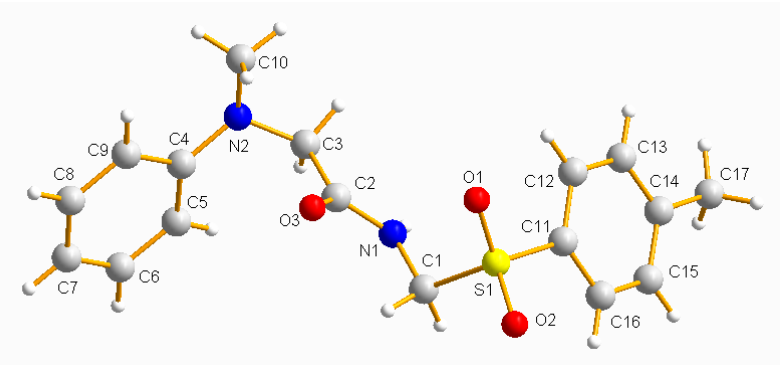

Figure S4. the structure of $\mathbf{3 a}$

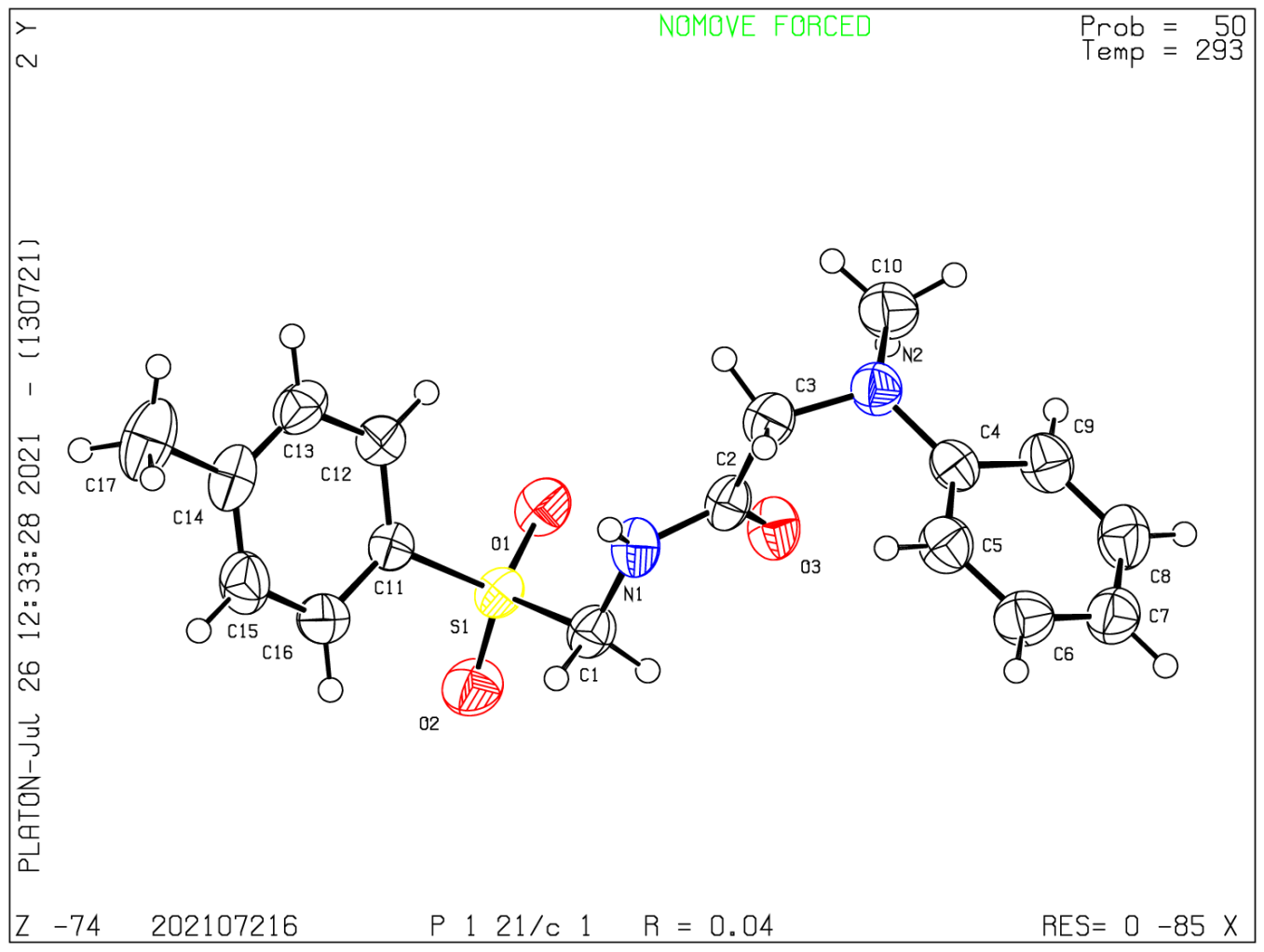

Figure S5. The structure of 3a (thermal ellipsoids at the 50\% probability level)

Table S8. Crystal data and structure refinement of 5d

\begin{tabular}{|c|c|}
\hline Structure & 5d \\
\hline Identification code & 202109224 \\
\hline Empirical formula & $\mathrm{C}_{18} \mathrm{H}_{19} \mathrm{BrN}_{2} \mathrm{O}_{3} \mathrm{~S}$ \\
\hline Formula weight & 423.32 \\
\hline Temperature/K & $293(2)$ \\
\hline Crystal system & monoclinic \\
\hline Space group & $\mathrm{P} 2{ }_{1} / \mathrm{c}$ \\
\hline
\end{tabular}




\begin{tabular}{|c|c|}
\hline $\mathrm{a} / \AA ̊ \AA$ & $10.5239(3)$ \\
\hline $\mathrm{b} / \AA$ & $17.6238(5)$ \\
\hline $\mathrm{c} / \AA$ & $10.02930(19)$ \\
\hline$\alpha /^{\circ}$ & 90 \\
\hline$\beta /{ }^{\circ}$ & $97.326(2)$ \\
\hline$\gamma /{ }^{\circ}$ & 90 \\
\hline Volume $/ \AA^{3}$ & $1844.95(8)$ \\
\hline $\mathrm{Z}$ & 4 \\
\hline$\rho_{\text {calc }} g / \mathrm{cm}^{3}$ & 1.524 \\
\hline$\mu / \mathrm{mm}^{-1}$ & 4.262 \\
\hline $\mathrm{F}(000)$ & 864.0 \\
\hline Crystal size $/ \mathrm{mm}^{3}$ & $0.462 \times 0.178 \times 0.033$ \\
\hline Radiation & $\operatorname{CuK} \alpha(\lambda=1.54184)$ \\
\hline $2 \Theta$ range for data collection $/{ }^{\circ}$ & 8.472 to 141.414 \\
\hline Index ranges & $-12 \leq \mathrm{h} \leq 12,-20 \leq \mathrm{k} \leq 21,-10 \leq 1 \leq 12$ \\
\hline Reflections collected & 7317 \\
\hline Independent reflections & $3478\left[\mathrm{R}_{\text {int }}=0.0407, \mathrm{R}_{\mathrm{sigma}}=0.0531\right]$ \\
\hline Data/restraints/parameters & $3478 / 1 / 231$ \\
\hline Goodness-of-fit on $\mathrm{F}^{2}$ & 1.033 \\
\hline Final $\mathrm{R}$ indexes $[\mathrm{I}>=2 \sigma(\mathrm{I})]$ & $\mathrm{R} 1=0.0520, \mathrm{wR}_{2}=0.1350$ \\
\hline Final $\mathrm{R}$ indexes [all data] & $\mathrm{R} 1=0.0724, \mathrm{wR} 2=0.1555$ \\
\hline Largest diff. peak/hole / e $\AA^{-3}$ & $0.45 /-0.41$ \\
\hline
\end{tabular}

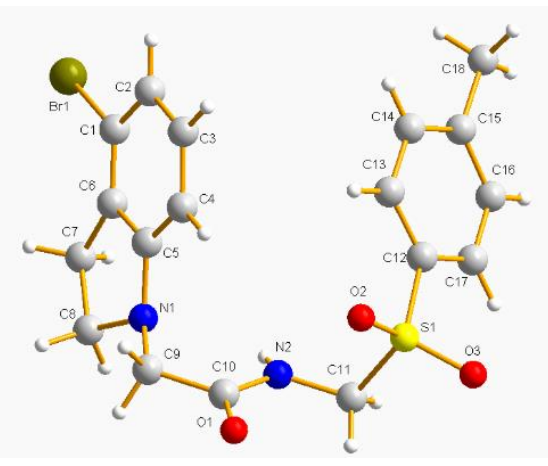

Figure S6. the structure of $\mathbf{5 d}$ 


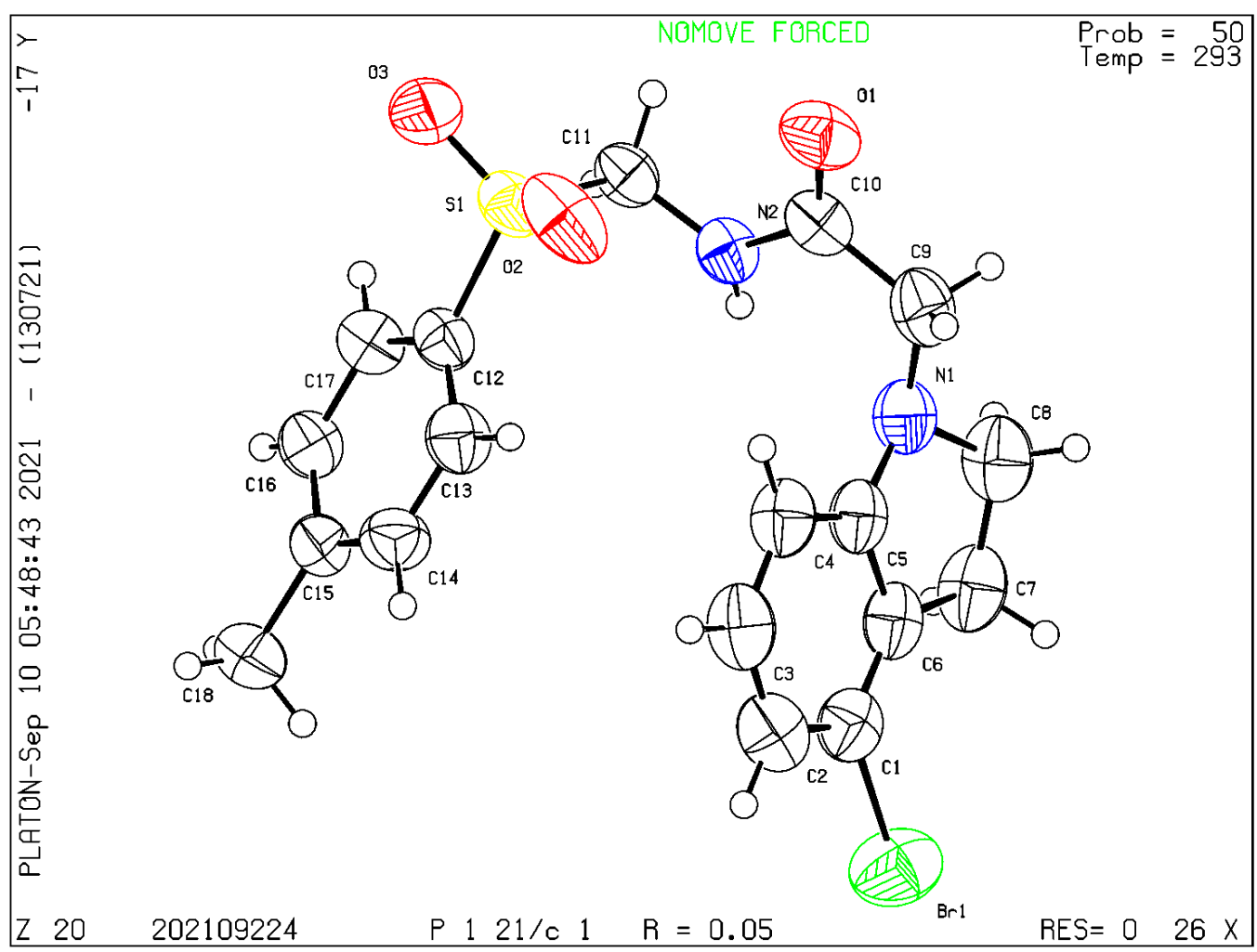

Figure S7. The structure of $\mathbf{5 d}$ (thermal ellipsoids at the $50 \%$ probability level)

Table S9. Crystal data and structure refinement of $\mathbf{5 m}$

\begin{tabular}{|c|c|}
\hline Structure & $\mathbf{5 m}$ \\
\hline Identification code & 201812500 \\
\hline Empirical formula & $\mathrm{C}_{14} \mathrm{H}_{20} \mathrm{~N}_{2} \mathrm{O}_{3} \mathrm{~S}_{2}$ \\
\hline Formula weight & 328.44 \\
\hline Temperature/K & $293(2)$ \\
\hline Crystal system & monoclinic \\
\hline Space group & $\mathrm{P} 2{ }_{1}$ \\
\hline $\mathrm{a} / \AA$ & $9.2135(9)$ \\
\hline $\mathrm{b} / \AA$ & $10.7842(6)$ \\
\hline $\mathrm{c} / \AA$ & $9.2288(9)$ \\
\hline$\alpha /{ }^{\circ}$ & 90 \\
\hline$\beta /{ }^{\circ}$ & $118.226(12)$ \\
\hline$\gamma^{\circ}$ & 90 \\
\hline $\mathrm{Volume} / \AA^{3}$ & $807.94(14)$ \\
\hline $\mathrm{Z}$ & 2 \\
\hline$\rho_{\text {calcg }} / \mathrm{cm}^{3}$ & 1.350 \\
\hline$\mu / \mathrm{mm}^{-1}$ & 3.087 \\
\hline $\mathrm{F}(000)$ & 348.0 \\
\hline & \\
\hline & \\
\hline
\end{tabular}




\begin{tabular}{|c|c|}
\hline Crystal size/mm $^{3}$ & $0.16 \times 0.13 \times 0.1$ \\
\hline Radiation & $\mathrm{CuK \alpha}(\lambda=1.54184)$ \\
\hline $2 \Theta$ range for data collection/ & 10.88 to 142.73 \\
\hline Index ranges & $-11 \leq \mathrm{h} \leq 10,-11 \leq \mathrm{k} \leq 13,-10 \leq 1 \leq 11$ \\
\hline Reflections collected & $2746\left[\mathrm{R}_{\text {int }}=0.0388, \mathrm{R}_{\text {sigma }}=0.0476\right]$ \\
\hline Independent reflections & $2746 / 1 / 195$ \\
\hline Data/restraints/parameters & 1.051 \\
\hline Goodness-of-fit on $\mathrm{F}^{2}$ & $\mathrm{R} 1=0.0430, \mathrm{wR}_{2}=0.1124$ \\
\hline Final $\mathrm{R}$ indexes $[\mathrm{I}>=2 \sigma(\mathrm{I})]$ & $\mathrm{R} 1=0.0483, \mathrm{wR} 2=0.1176$ \\
\hline Final $\mathrm{R}$ indexes [all data] & $0.35 /-0.27$ \\
\hline Largest diff. peak/hole $/ \mathrm{e} \AA^{-3}$ & \\
\hline &
\end{tabular}

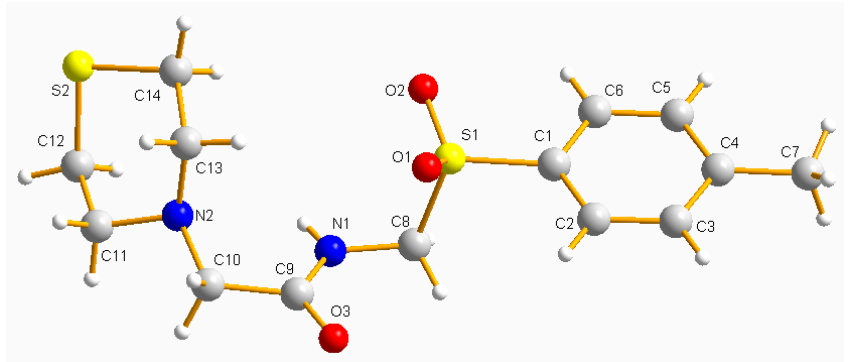

Figure S8. the structure of $\mathbf{5 m}$

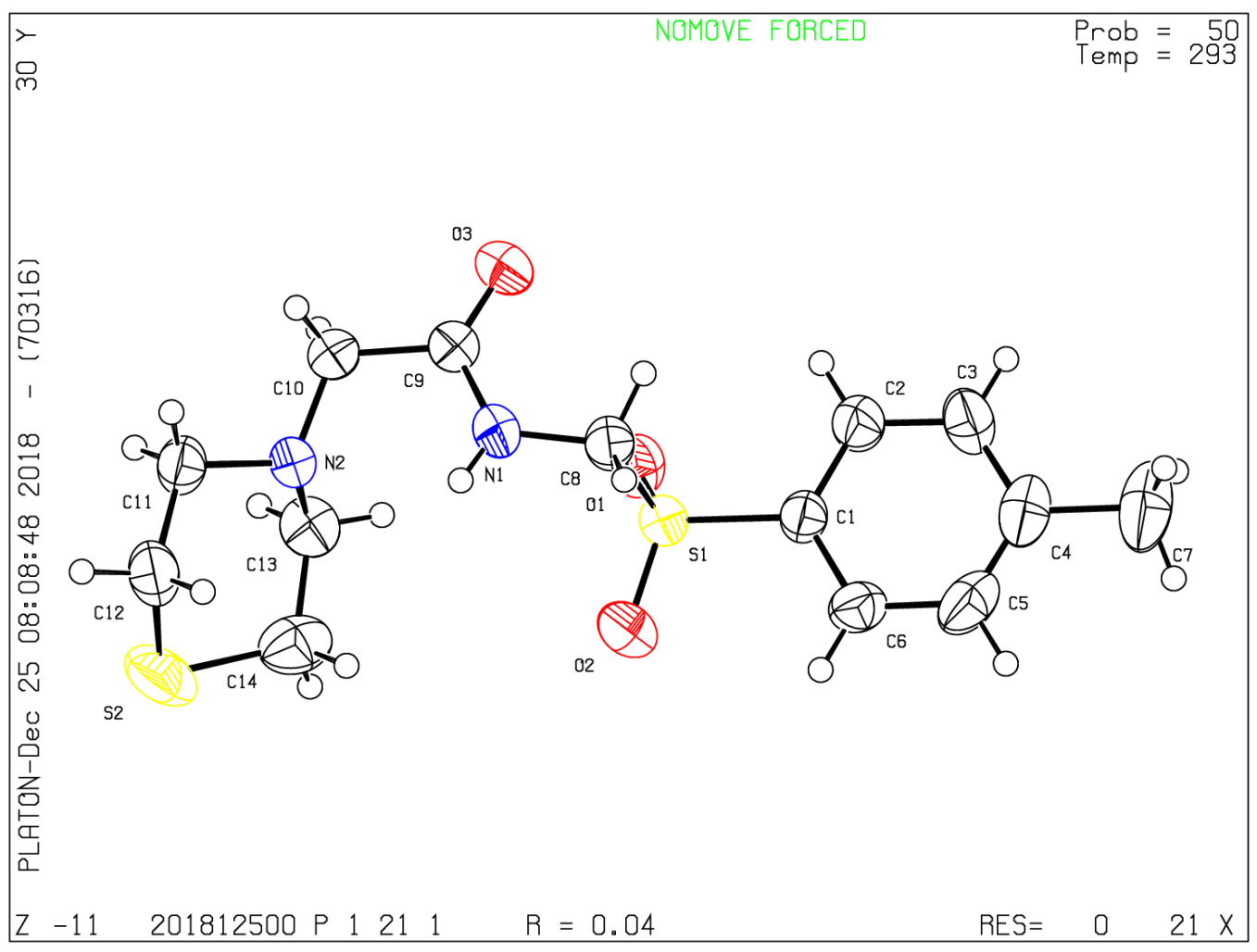

Figure S9. The structure of $\mathbf{5 m}$ (thermal ellipsoids at the $50 \%$ probability level) 
Table S10. Crystal data and structure refinement of $\mathbf{5 n}$

\begin{tabular}{|c|c|}
\hline Structure & $5 n$ \\
\hline Identification code & 201812501 \\
\hline Empirical formula & $\mathrm{C}_{19} \mathrm{H}_{22} \mathrm{~N}_{2} \mathrm{O}_{3} \mathrm{~S}$ \\
\hline Formula weight & 358.44 \\
\hline Temperature/K & 293(2) \\
\hline Crystal system & orthorhombic \\
\hline Space group & Pbca \\
\hline $\mathrm{a} / \AA$ & $16.1637(4)$ \\
\hline $\mathrm{b} / \AA$ & $9.98046(18)$ \\
\hline $\mathrm{c} / \AA$ & $23.8656(6)$ \\
\hline$\alpha /^{\circ}$ & 90 \\
\hline$\beta /^{\circ}$ & 90 \\
\hline$\gamma /{ }^{\circ}$ & 90 \\
\hline Volume $/ \AA^{3}$ & $3850.03(14)$ \\
\hline $\mathrm{Z}$ & 8 \\
\hline$\rho_{\text {calc }} \mathrm{g} / \mathrm{cm}^{3}$ & 1.237 \\
\hline$\mu / \mathrm{mm}^{-1}$ & 1.652 \\
\hline $\mathrm{F}(000)$ & 1520.0 \\
\hline Crystal size $/ \mathrm{mm}^{3}$ & $0.15 \times 0.13 \times 0.05$ \\
\hline Radiation & $\operatorname{CuK} \alpha(\lambda=1.54184)$ \\
\hline $2 \Theta$ range for data collection $/{ }^{\circ}$ & 7.408 to 134.148 \\
\hline Index ranges & $-19 \leq \mathrm{h} \leq 19,-7 \leq \mathrm{k} \leq 11,-28 \leq 1 \leq 27$ \\
\hline Reflections collected & 27635 \\
\hline Independent reflections & $3434\left[\mathrm{R}_{\text {int }}=0.0414, \mathrm{R}_{\text {sigma }}=0.0189\right]$ \\
\hline Data/restraints/parameters & $3434 / 0 / 231$ \\
\hline Goodness-of-fit on $\mathrm{F}^{2}$ & 1.042 \\
\hline Final $\mathrm{R}$ indexes $[\mathrm{I}>=2 \sigma(\mathrm{I})]$ & $\mathrm{R} 1=0.0478, \mathrm{wR}_{2}=0.1380$ \\
\hline Final $\mathrm{R}$ indexes [all data] & $\mathrm{R} 1=0.0566, \mathrm{wR} 2=0.1479$ \\
\hline Largest diff. peak/hole / e $\AA^{-3}$ & $0.29 /-0.18$ \\
\hline
\end{tabular}

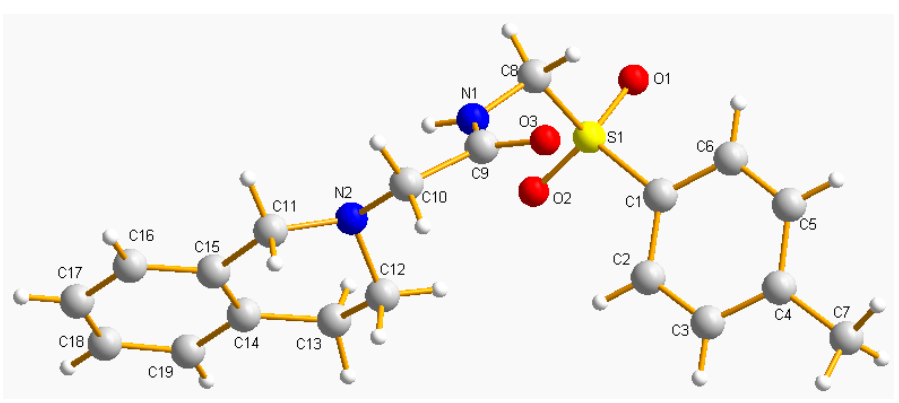

Figure S10. the structure of $\mathbf{5 n}$ 


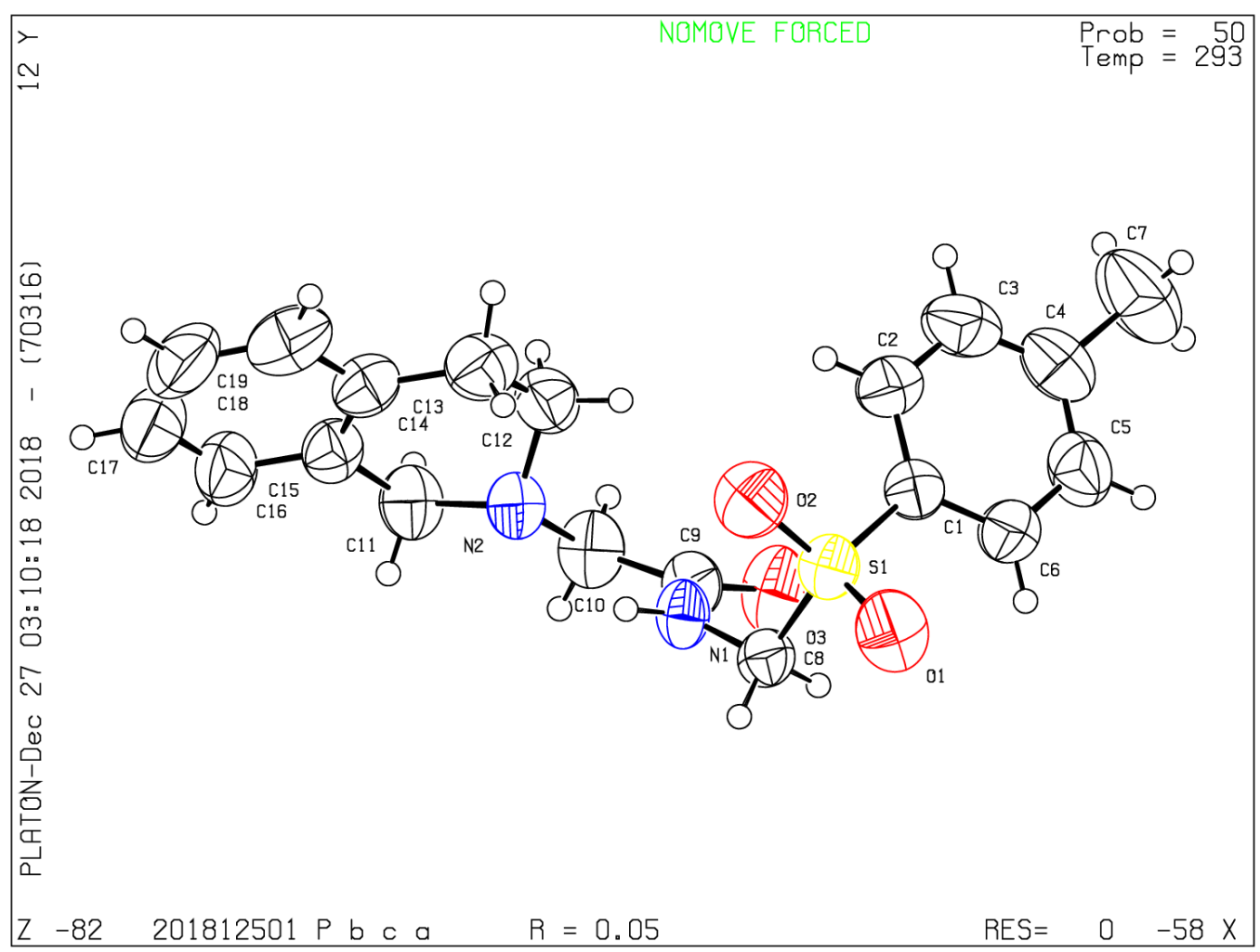

Figure S11. The structure of $\mathbf{5 n}$ (thermal ellipsoids at the 50\% probability level) 


\section{NMR Spectra of Compounds}

${ }^{1} \mathrm{H}$ NMR (600 MHz, $\mathrm{CDCl}_{3}$ ) of compound 3a

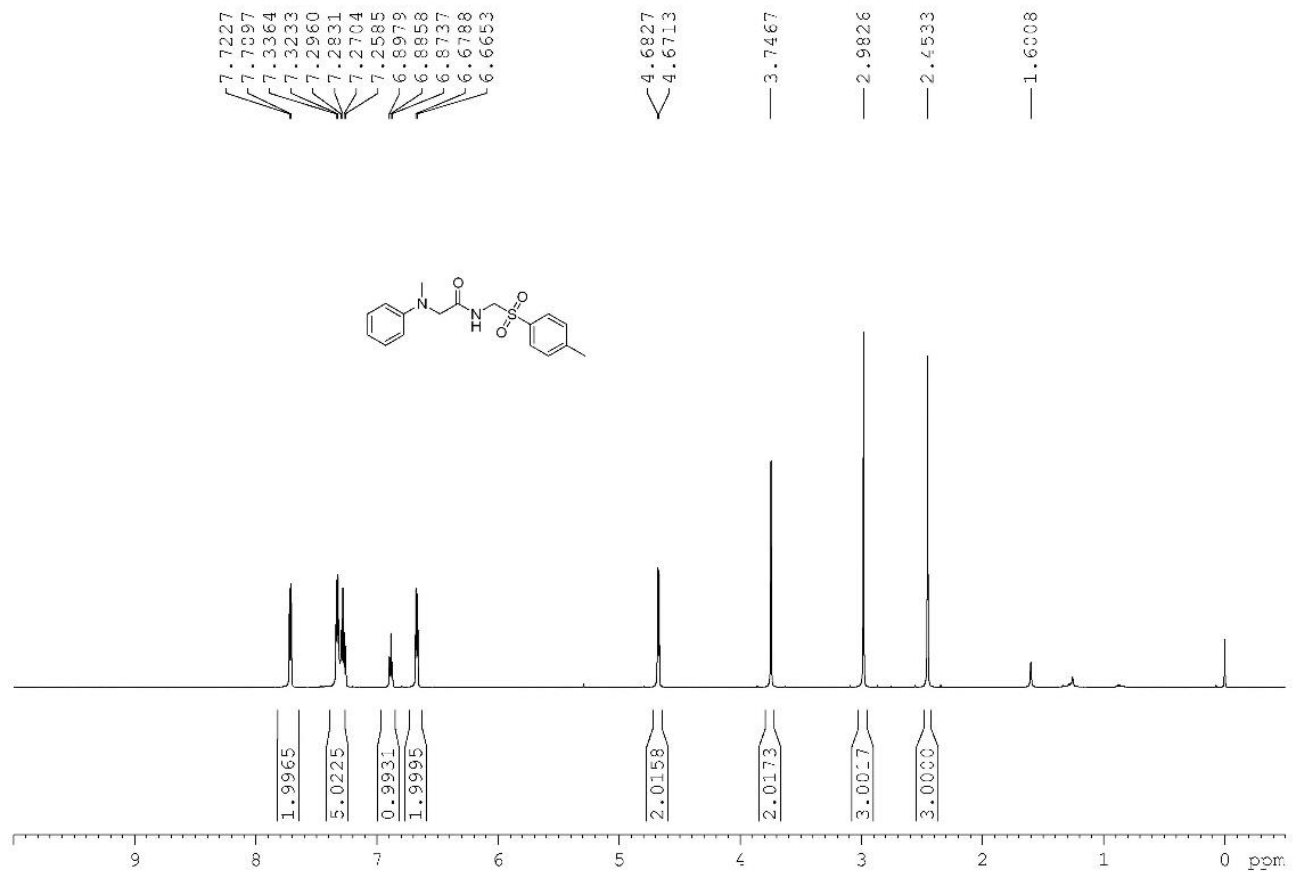

${ }^{13} \mathrm{C}\left\{{ }^{1} \mathrm{H}\right\}$ NMR (151 MHz, $\left.\mathrm{CDCl}_{3}\right)$ of compound 3a

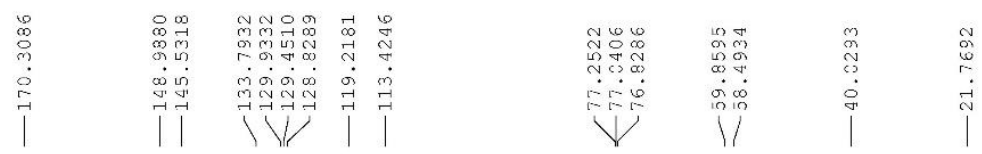
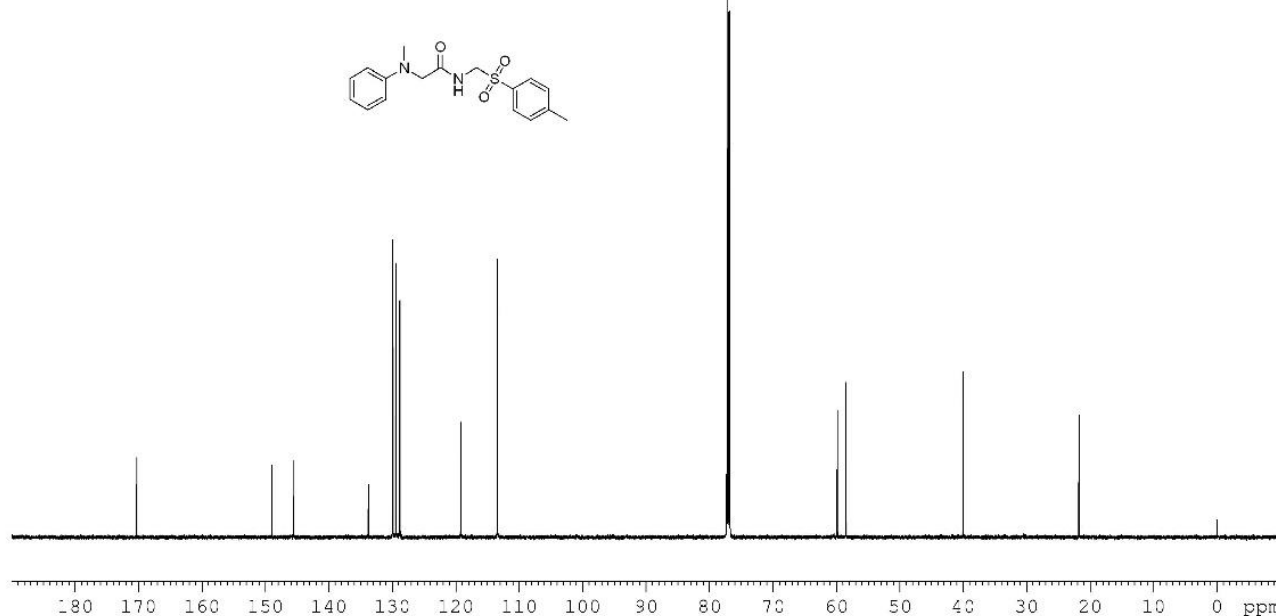
${ }^{1} \mathrm{H}$ NMR $\left(600 \mathrm{MHz}, \mathrm{CDCl}_{3}\right)$ of compound $\mathbf{3 b}$

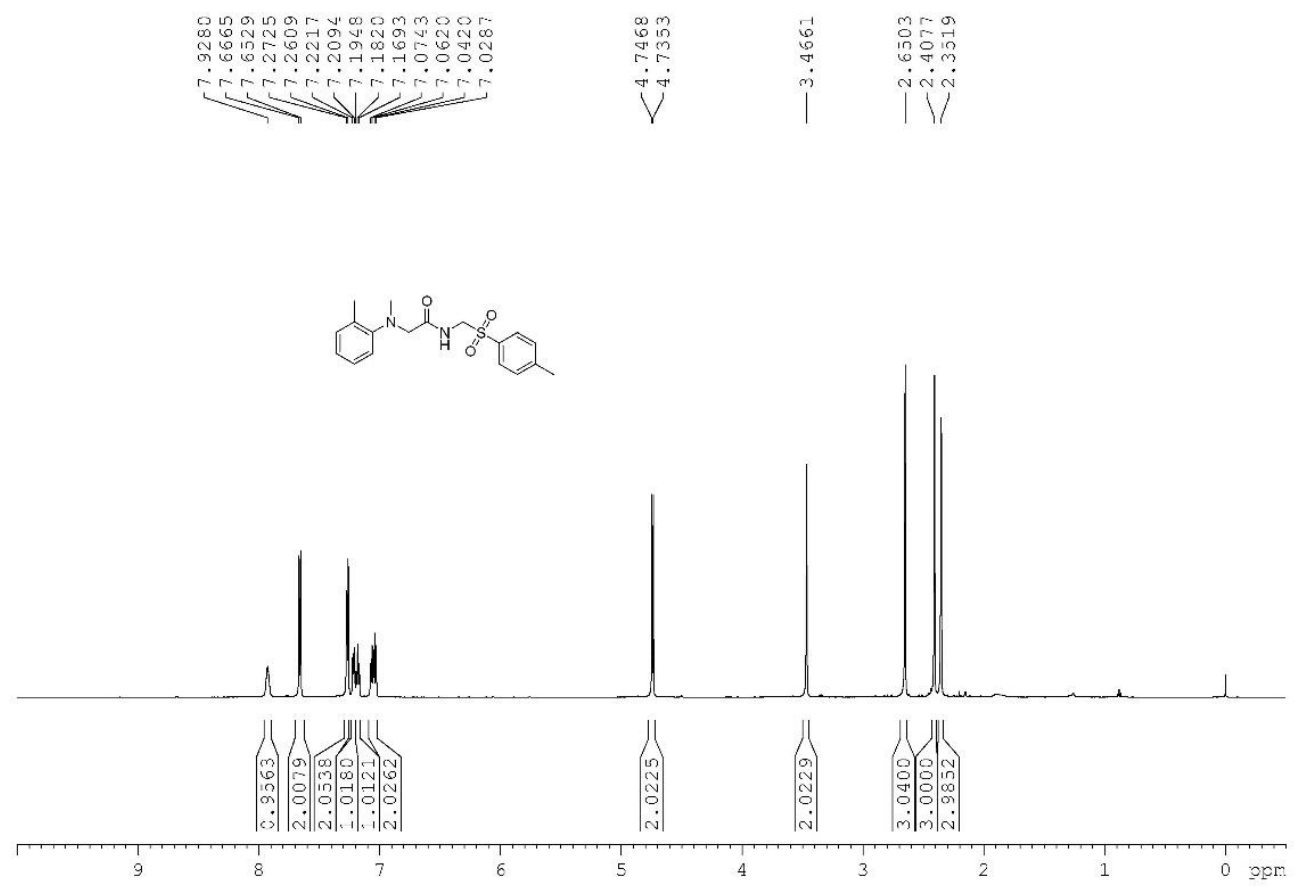

${ }^{13} \mathrm{C}\left\{{ }^{1} \mathrm{H}\right\}$ NMR (101 MHz, $\left.\mathrm{CDCl}_{3}\right)$ of compound $\mathbf{3 b}$

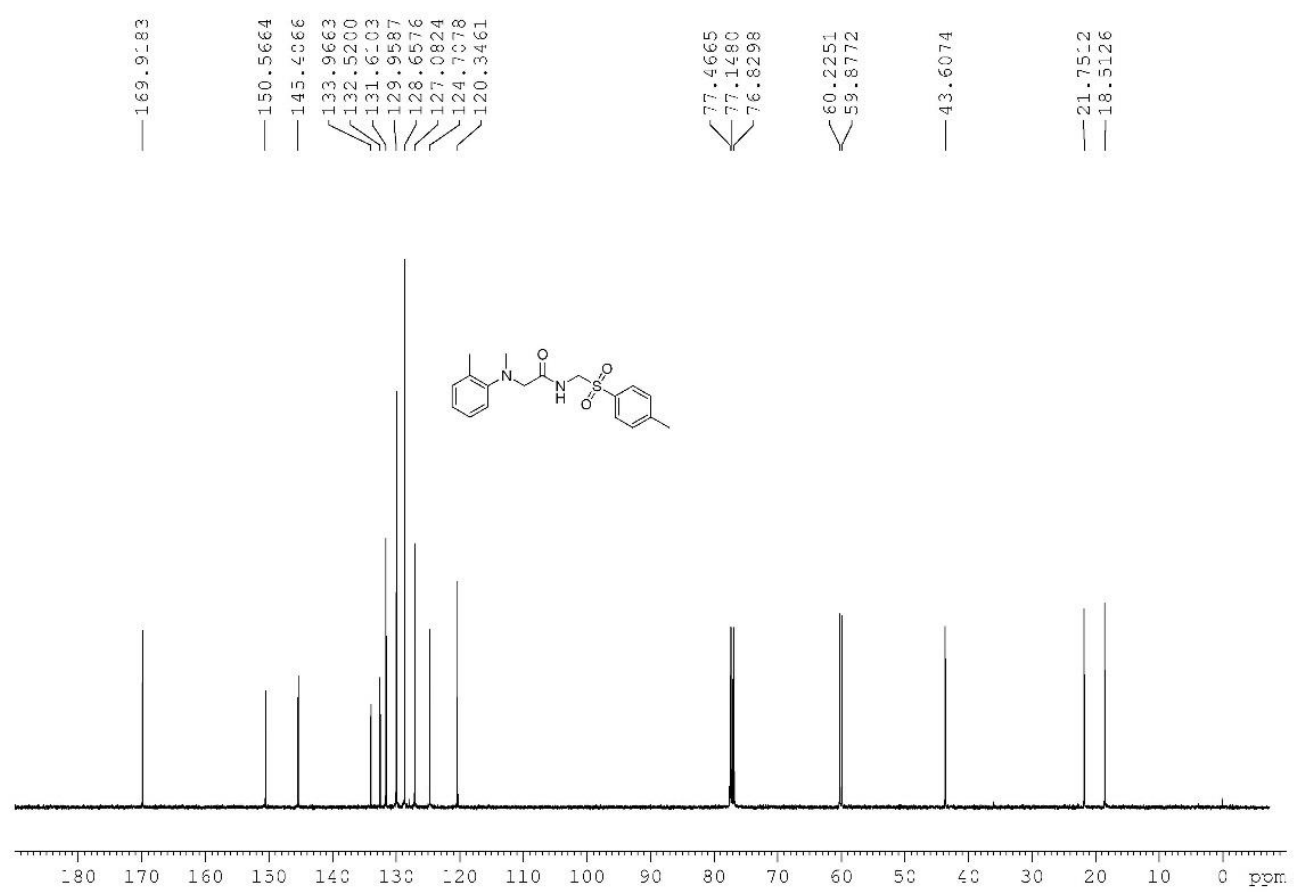


${ }^{1} \mathrm{H}$ NMR $\left(600 \mathrm{MHz}, \mathrm{CDCl}_{3}\right)$ of compound $\mathbf{3 c}$

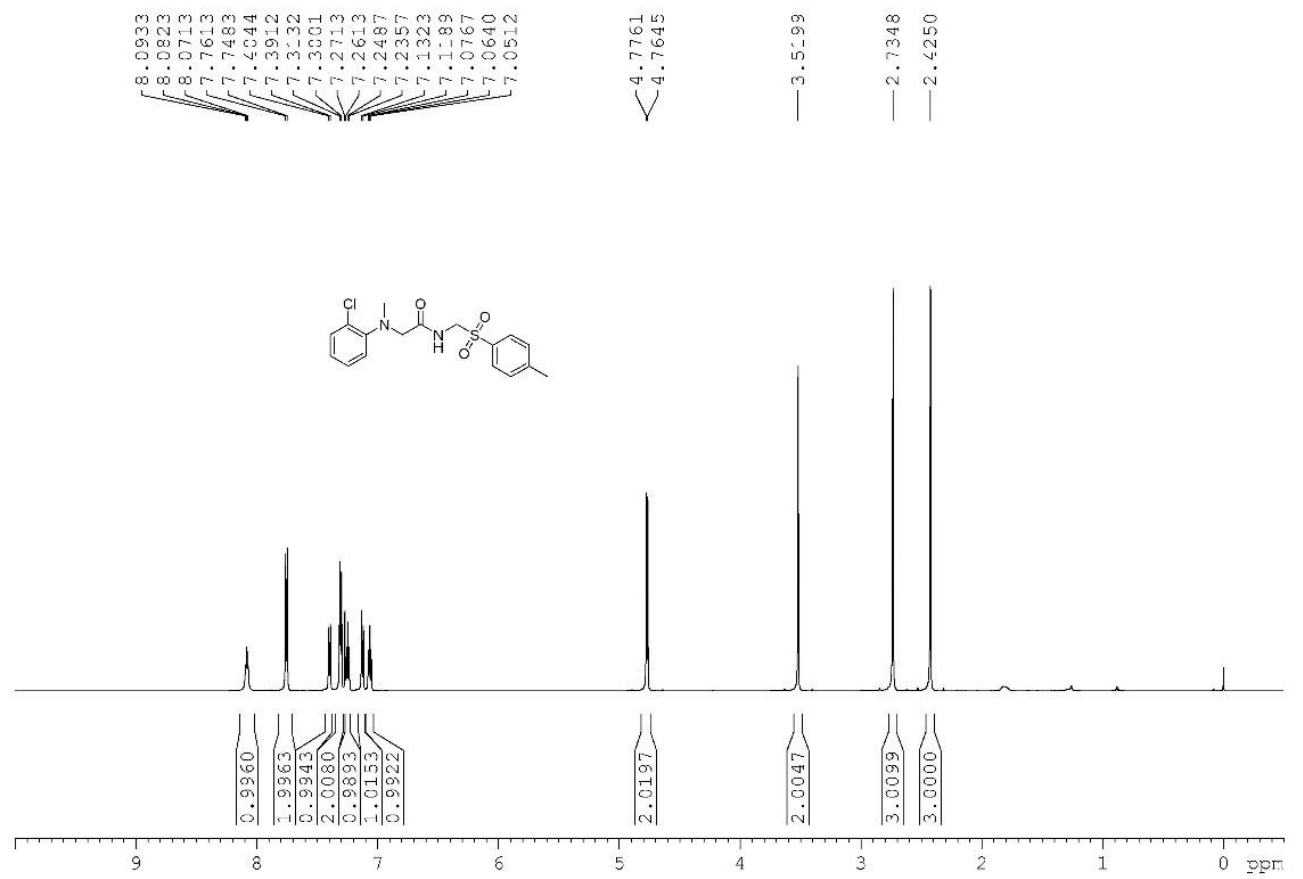

${ }^{13} \mathrm{C}\left\{{ }^{1} \mathrm{H}\right\}$ NMR (101 MHz, $\mathrm{CDCl} 3$ ) of compound $\mathbf{3 c}$
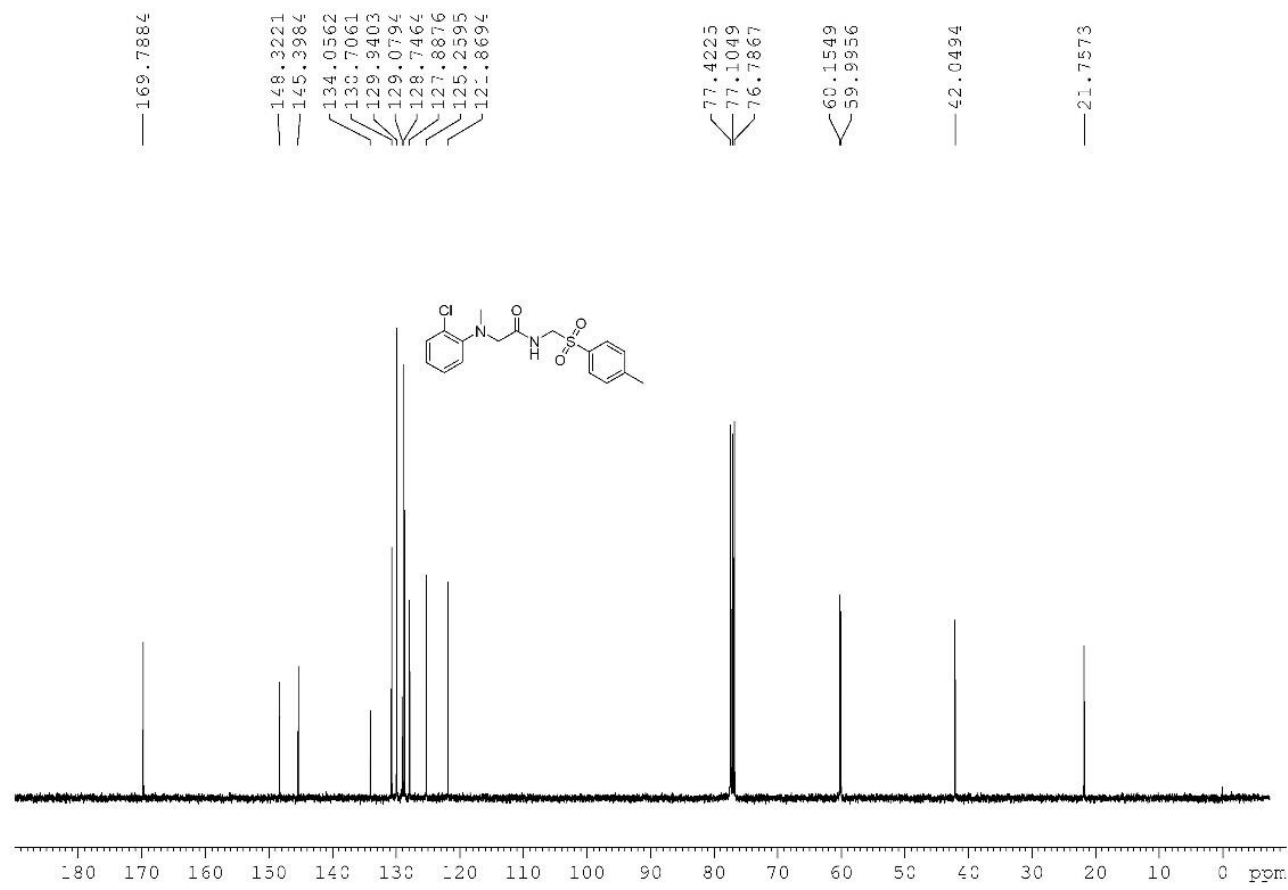
${ }^{1} \mathrm{H}$ NMR $\left(600 \mathrm{MHz}, \mathrm{CDCl}_{3}\right)$ of compound $\mathbf{3 d}$
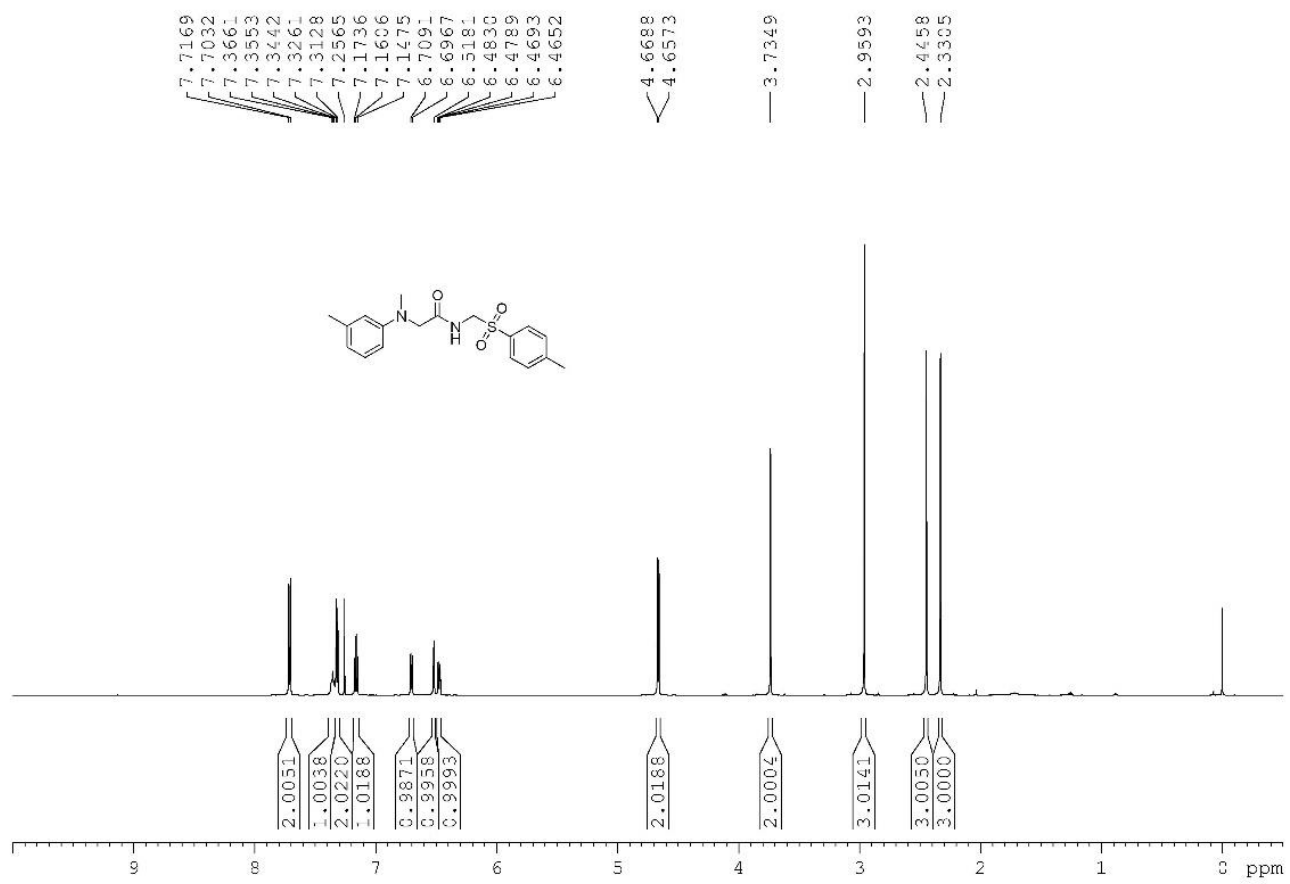

${ }^{13} \mathrm{C}\left\{{ }^{1} \mathrm{H}\right\}$ NMR (151 MHz, $\left.\mathrm{CDCl}_{3}\right)$ of compound $\mathbf{3 d}$
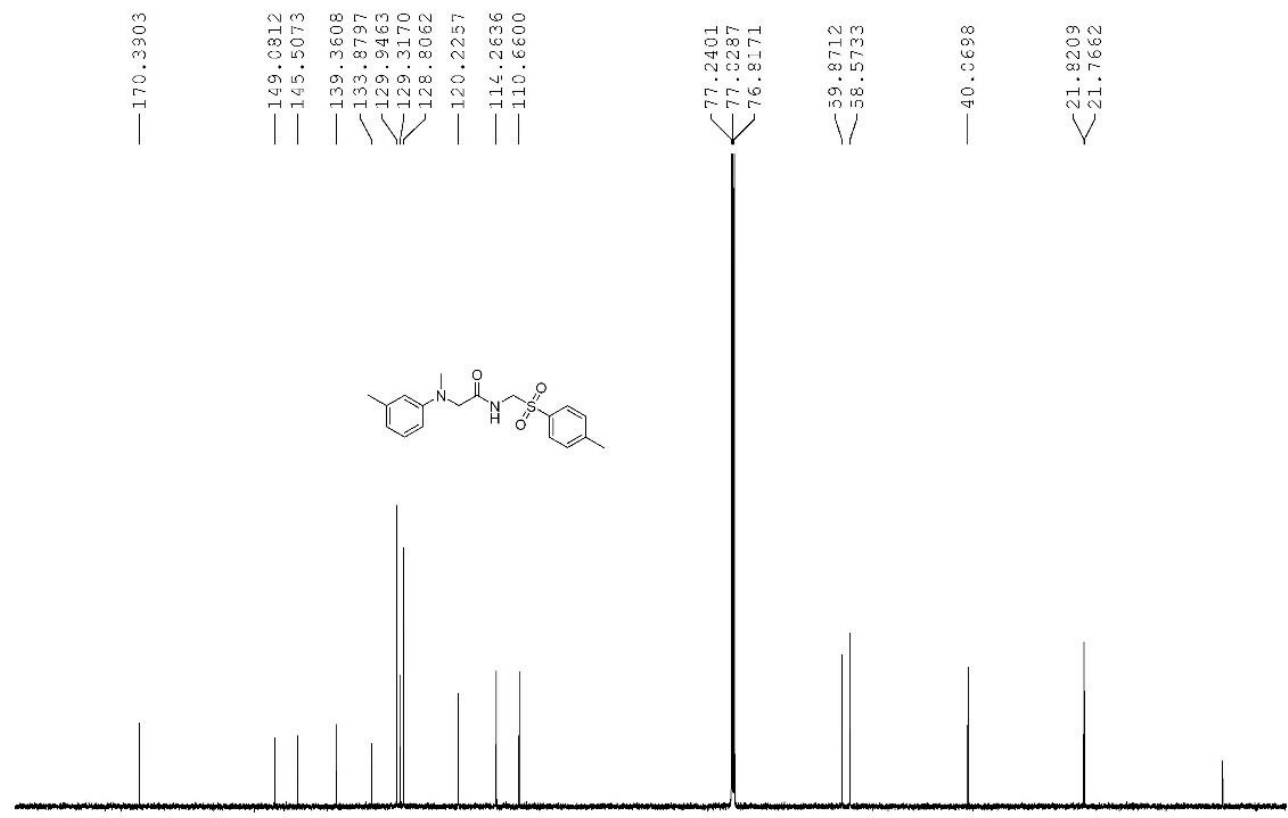

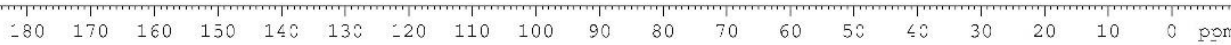


${ }^{1} \mathrm{H}$ NMR (600 MHz, $\mathrm{CDCl}_{3}$ ) of compound 3e
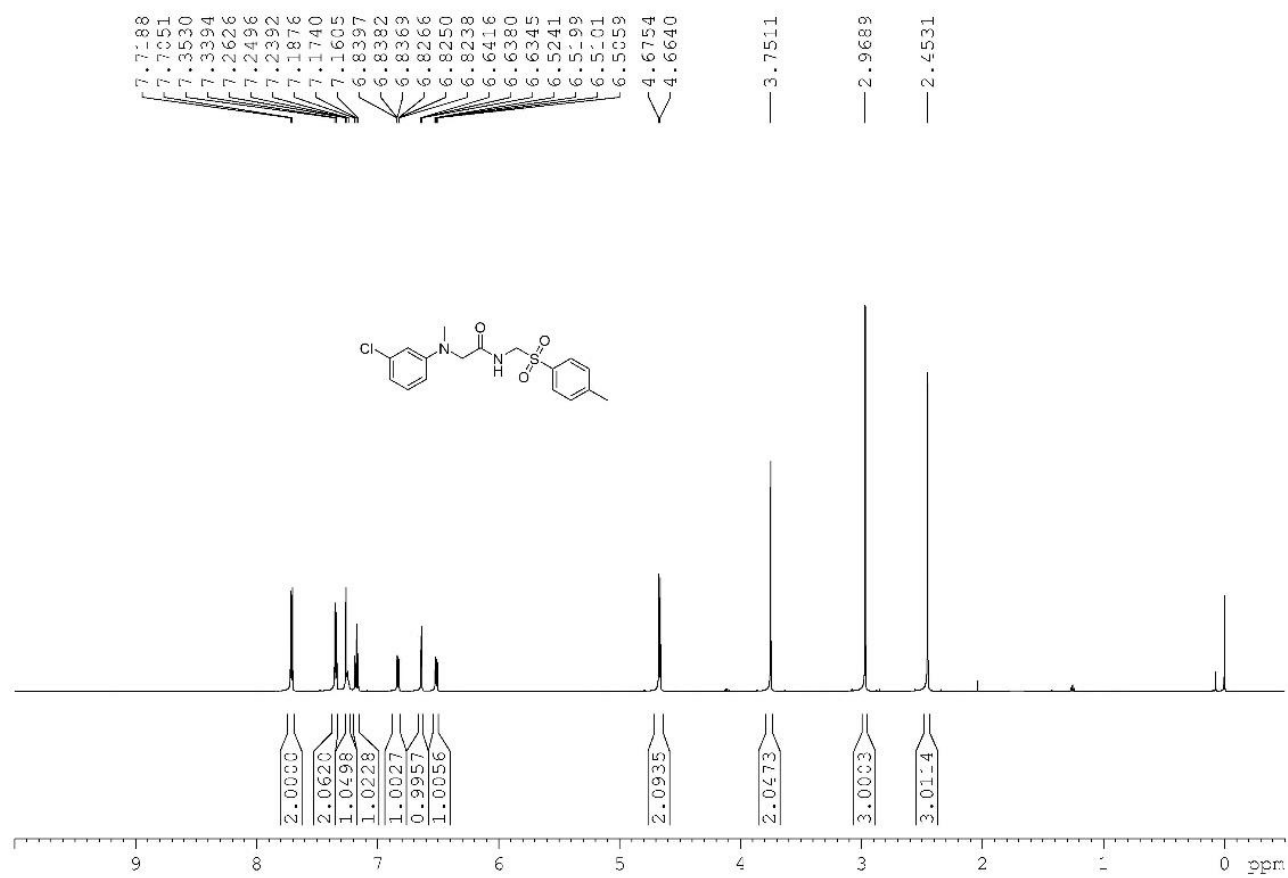

${ }^{13} \mathrm{C}\left\{{ }^{1} \mathrm{H}\right\}$ NMR (151 MHz, $\left.\mathrm{CDCl}_{3}\right)$ of compound $3 \mathbf{e}$
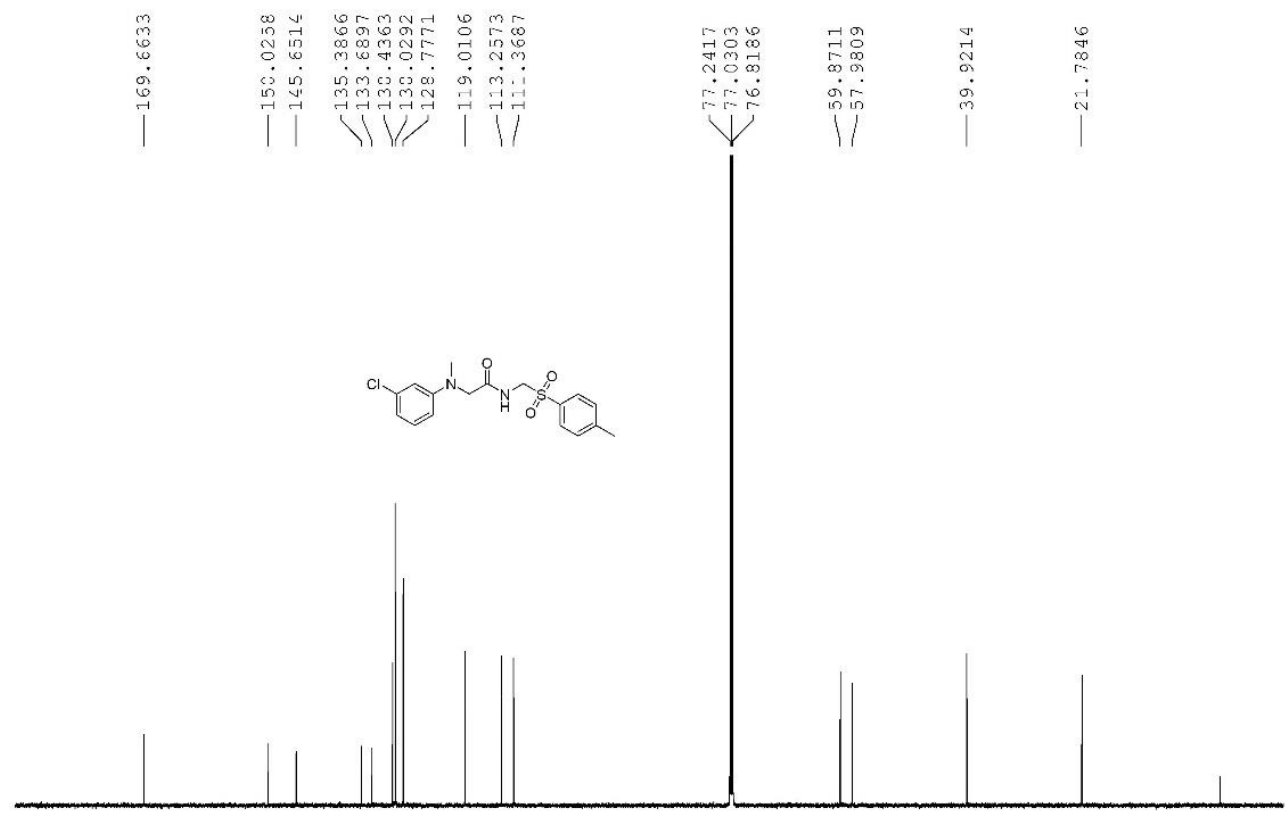

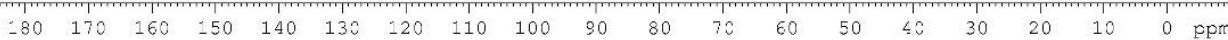


${ }^{1} \mathrm{H}$ NMR $\left(600 \mathrm{MHz}, \mathrm{CDCl}_{3}\right)$ of compound $\mathbf{3 f}$

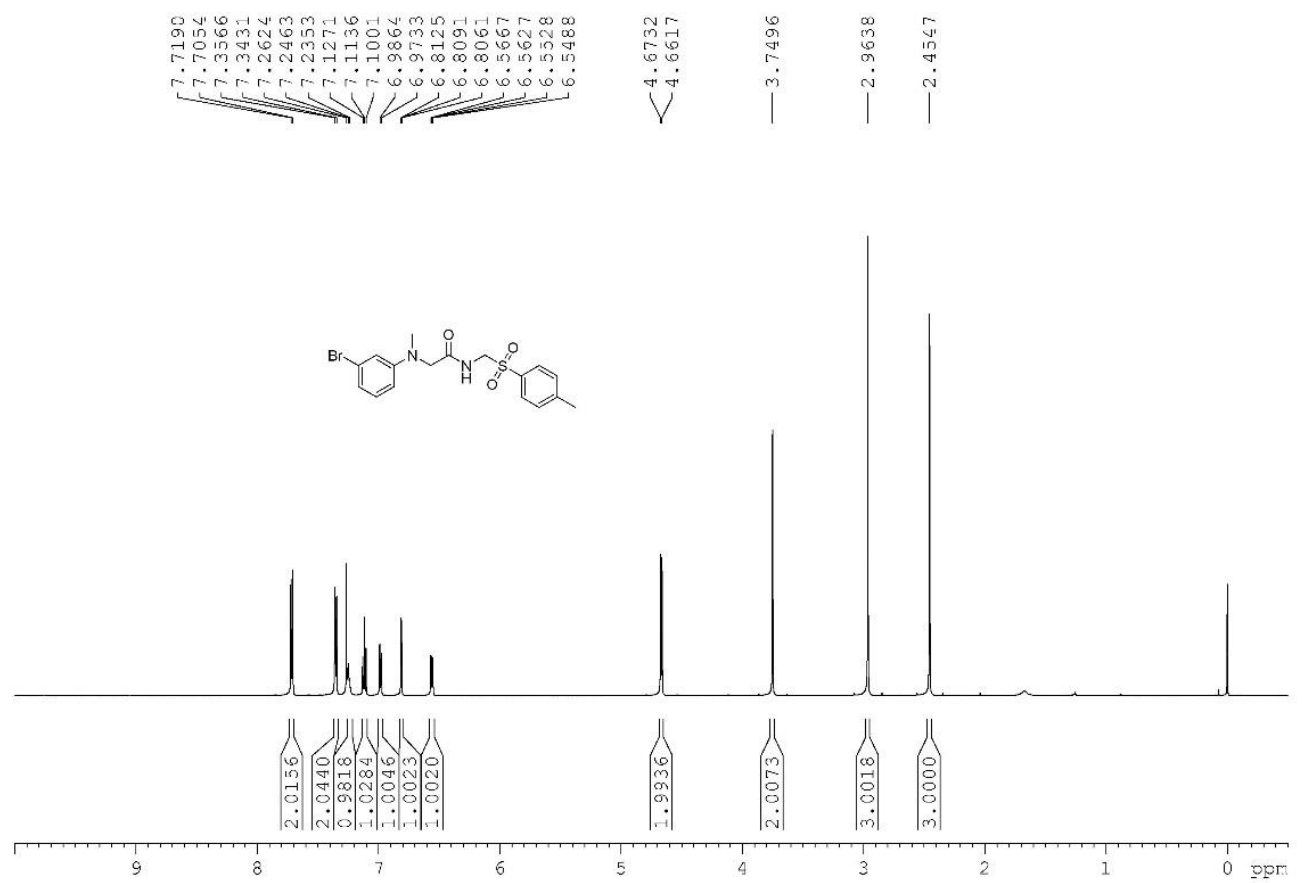

${ }^{13} \mathrm{C}\left\{{ }^{1} \mathrm{H}\right\}$ NMR (101 MHz, $\left.\mathrm{CDCl}_{3}\right)$ of compound $\mathbf{3 f}$
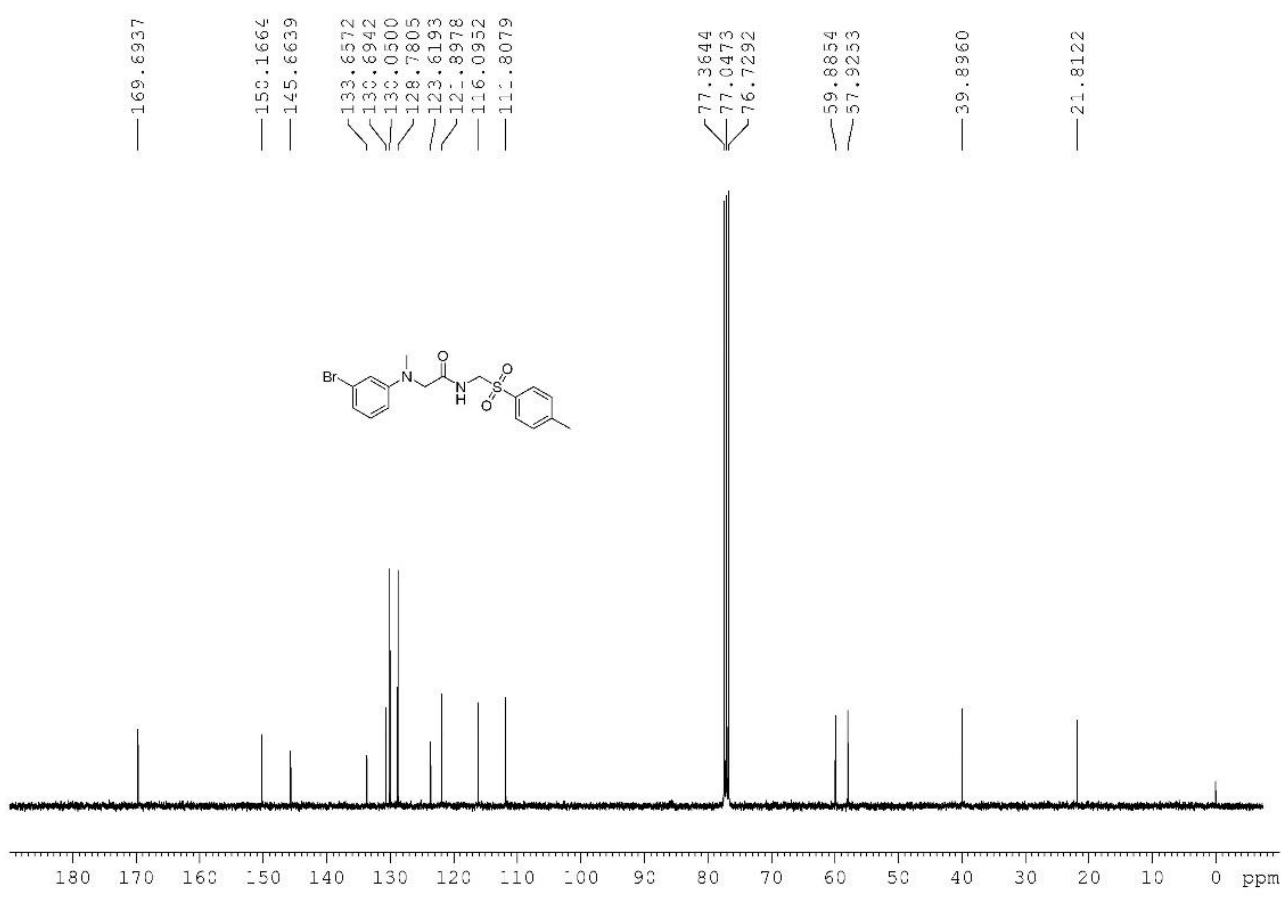
${ }^{1} \mathrm{H}$ NMR $\left(600 \mathrm{MHz}, \mathrm{CDCl}_{3}\right)$ of compound $\mathbf{3 g}$

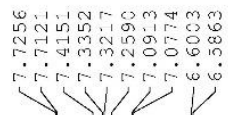

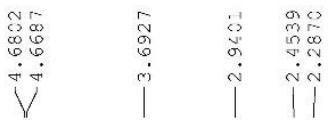

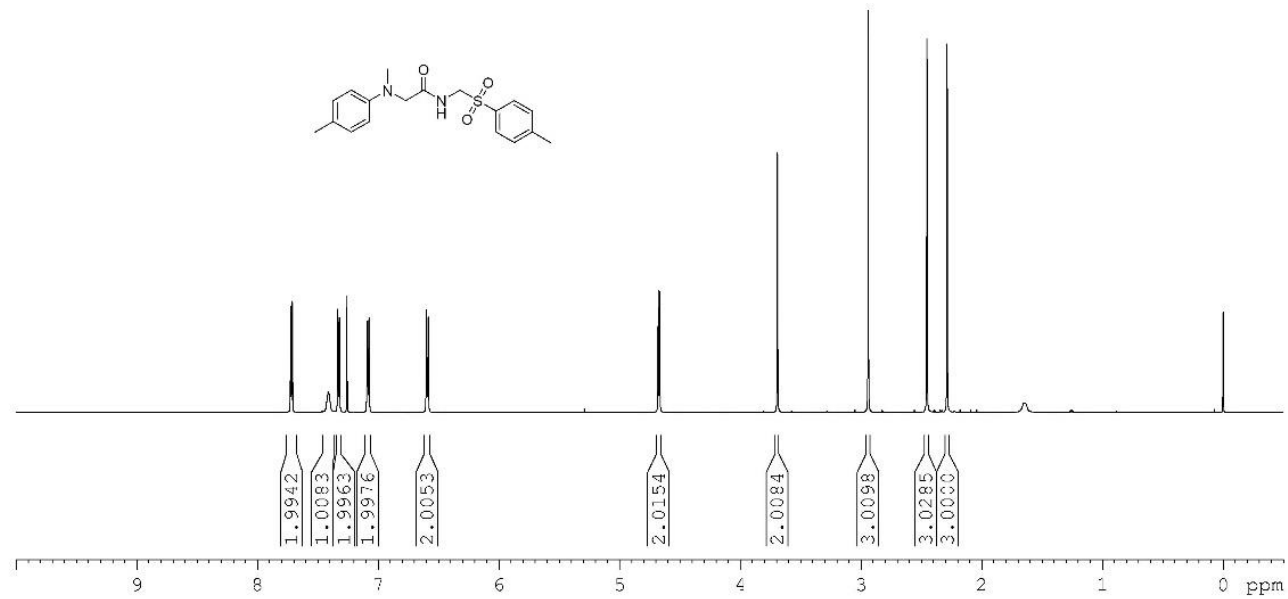

${ }^{13} \mathrm{C}\left\{{ }^{1} \mathrm{H}\right\}$ NMR (151 MHz, $\left.\mathrm{CDCl}_{3}\right)$ of compound $\mathbf{3 g}$

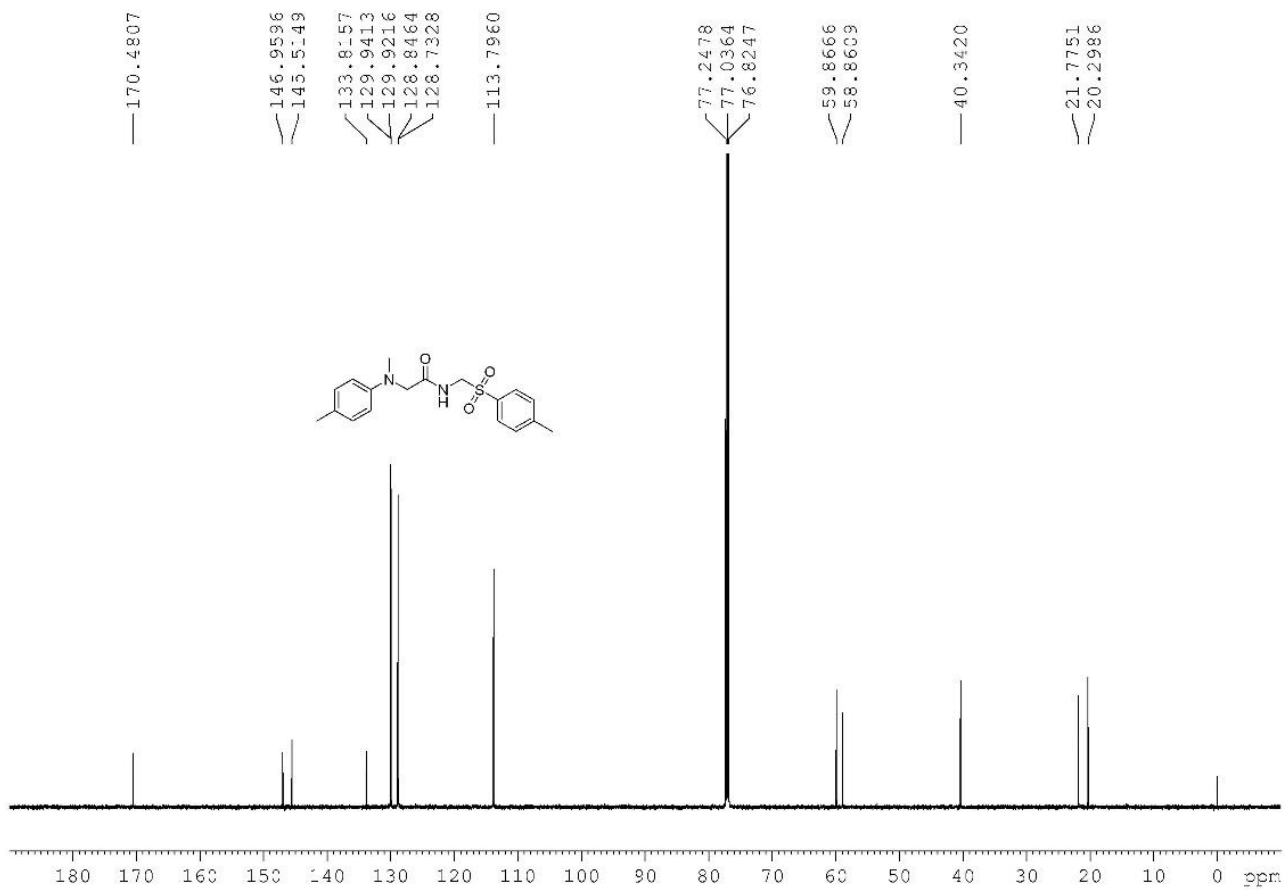


${ }^{1} \mathrm{H}$ NMR (400 MHz, $\mathrm{CDCl}_{3}$ ) of compound $\mathbf{3 h}$

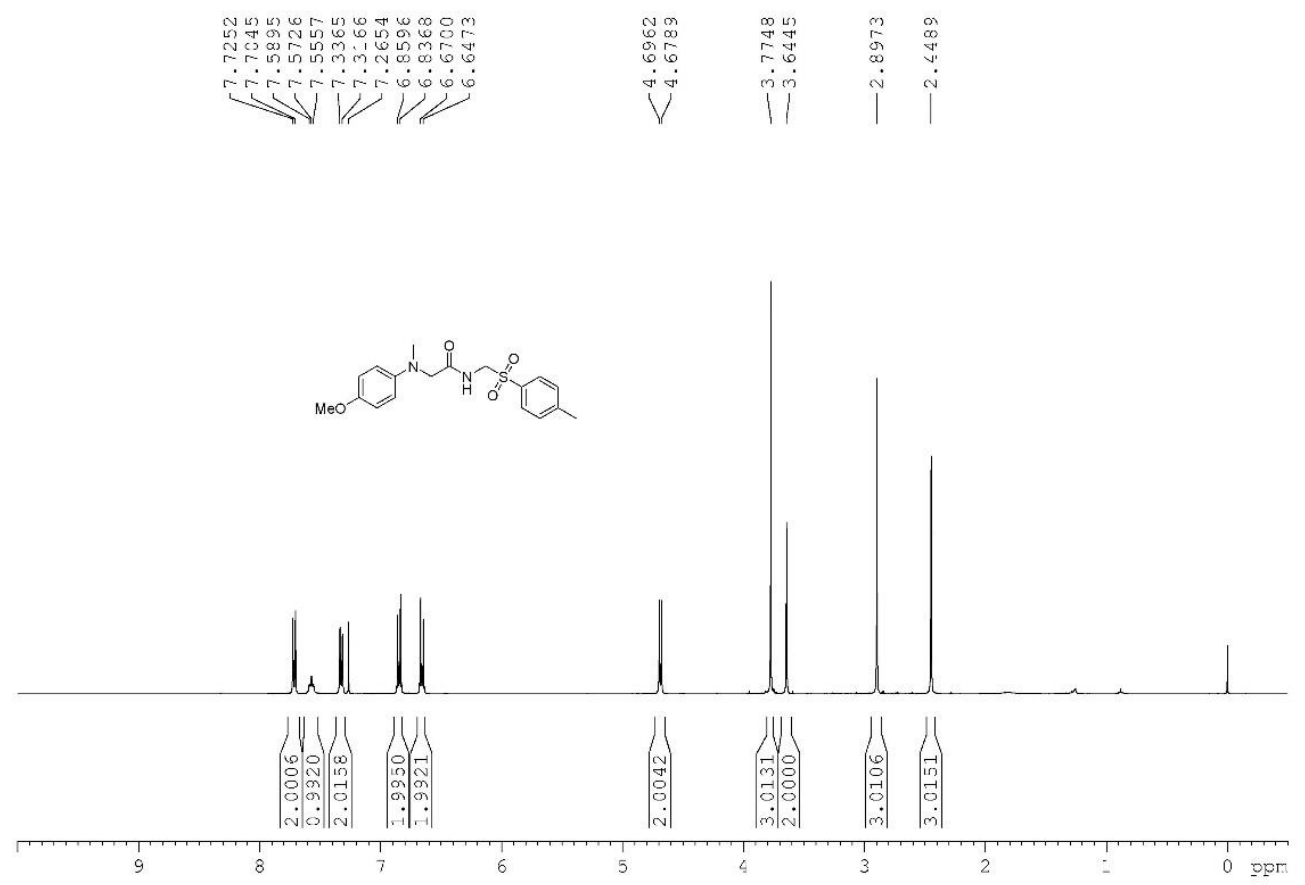

${ }^{13} \mathrm{C}\left\{{ }^{1} \mathrm{H}\right\}$ NMR $\left(101 \mathrm{MHz}, \mathrm{CDCl}_{3}\right)$ of compound $\mathbf{3 h}$
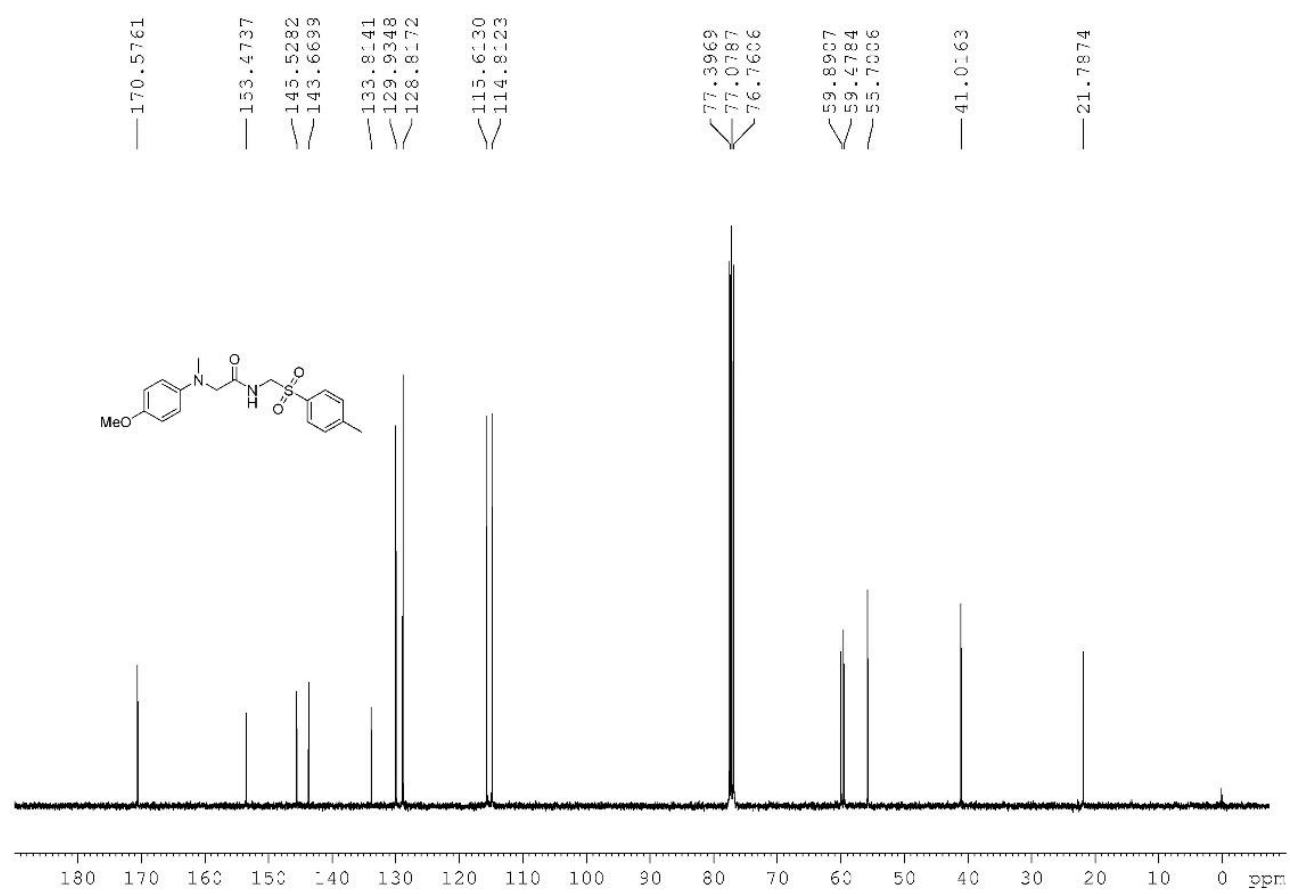
${ }^{1} \mathrm{H}$ NMR (600 MHz, $\mathrm{CDCl}_{3}$ ) of compound $\mathbf{3 i}$

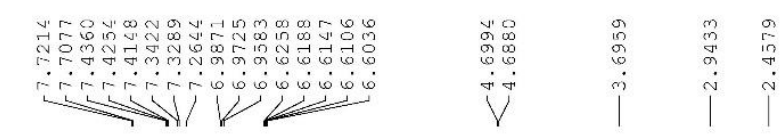

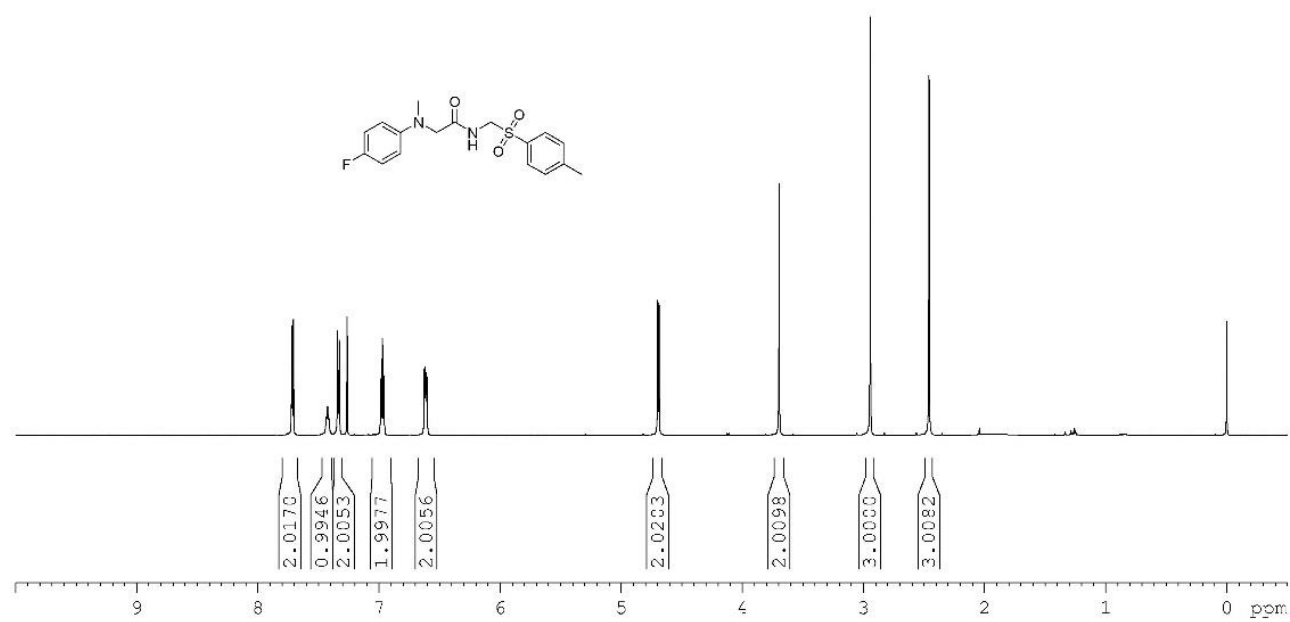

${ }^{13} \mathrm{C}\left\{{ }^{1} \mathrm{H}\right\}$ NMR $\left(101 \mathrm{MHz}, \mathrm{CDCl}_{3}\right)$ of compound $\mathbf{3 i}$

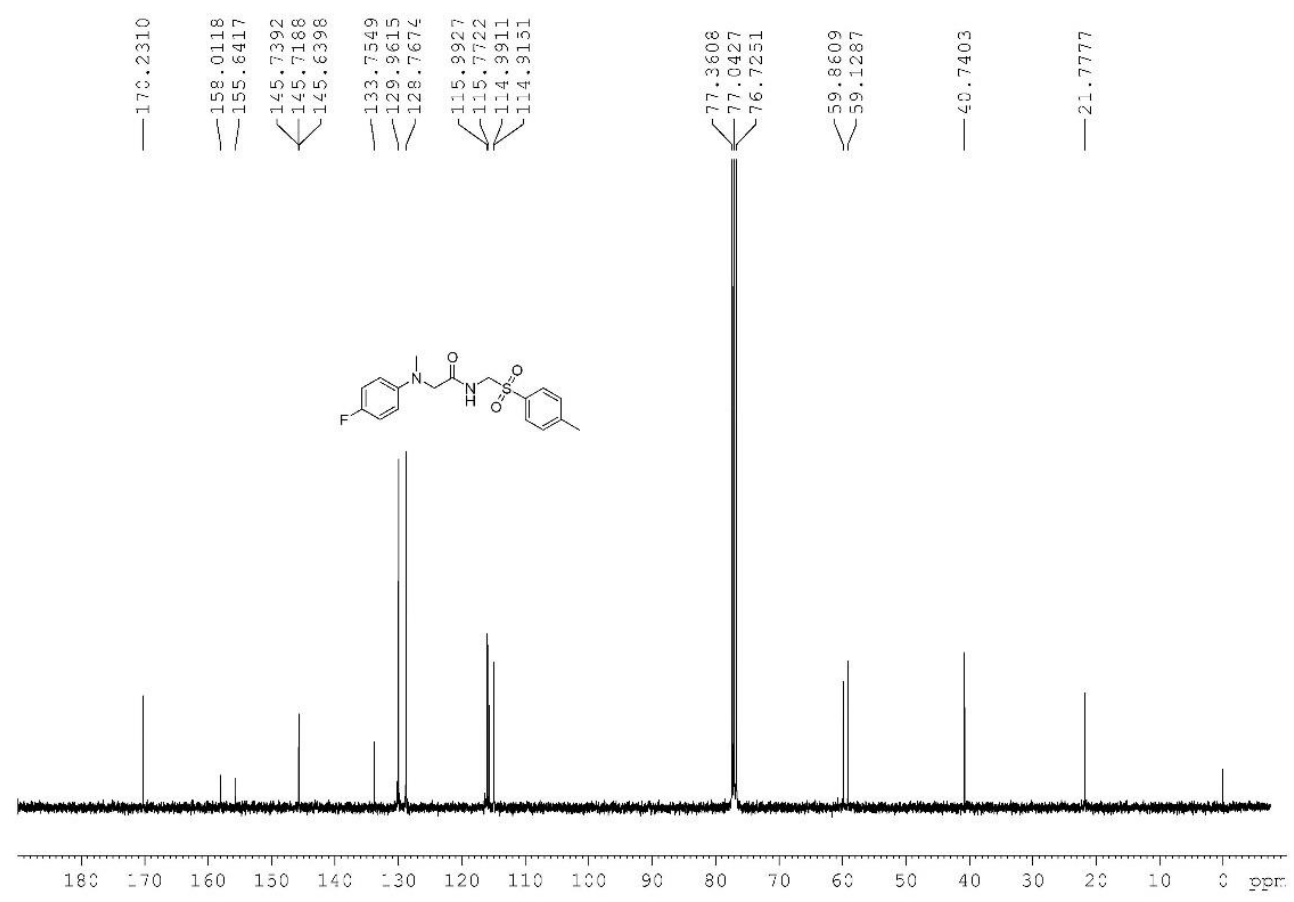


${ }^{19} \mathrm{~F}$ NMR (565 MHz, $\mathrm{CDCl}_{3}$ ) of compound 3i

$$
\begin{aligned}
& 9 \\
& 0 \\
& 0 \\
& 5 \\
& 15 \\
& 0 \\
& 7 \\
& 7 \\
& 1
\end{aligned}
$$<smiles>[AlH2]</smiles>

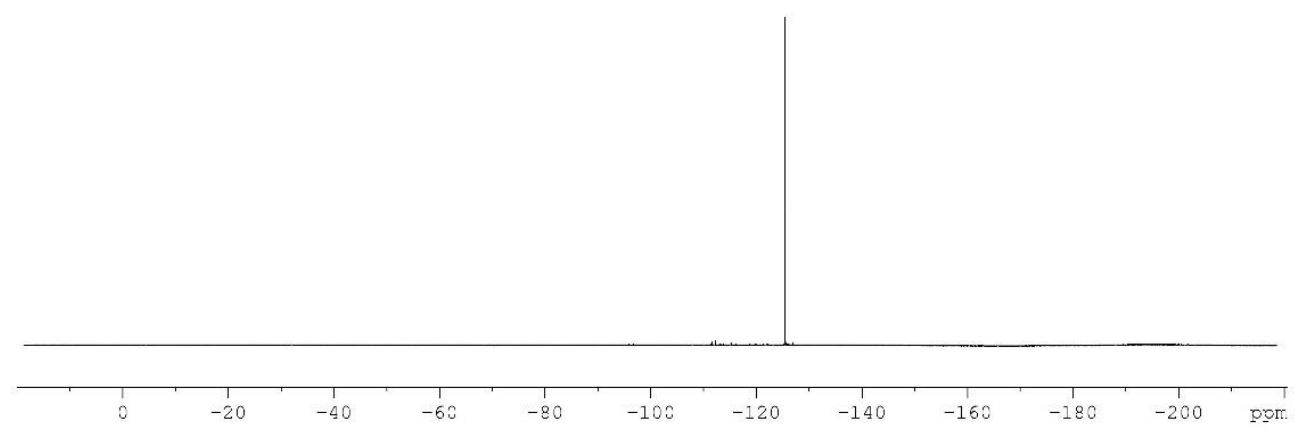

${ }^{1} \mathrm{H}$ NMR (600 MHz, $\mathrm{CDCl}_{3}$ ) of compound $\mathbf{3 j}$

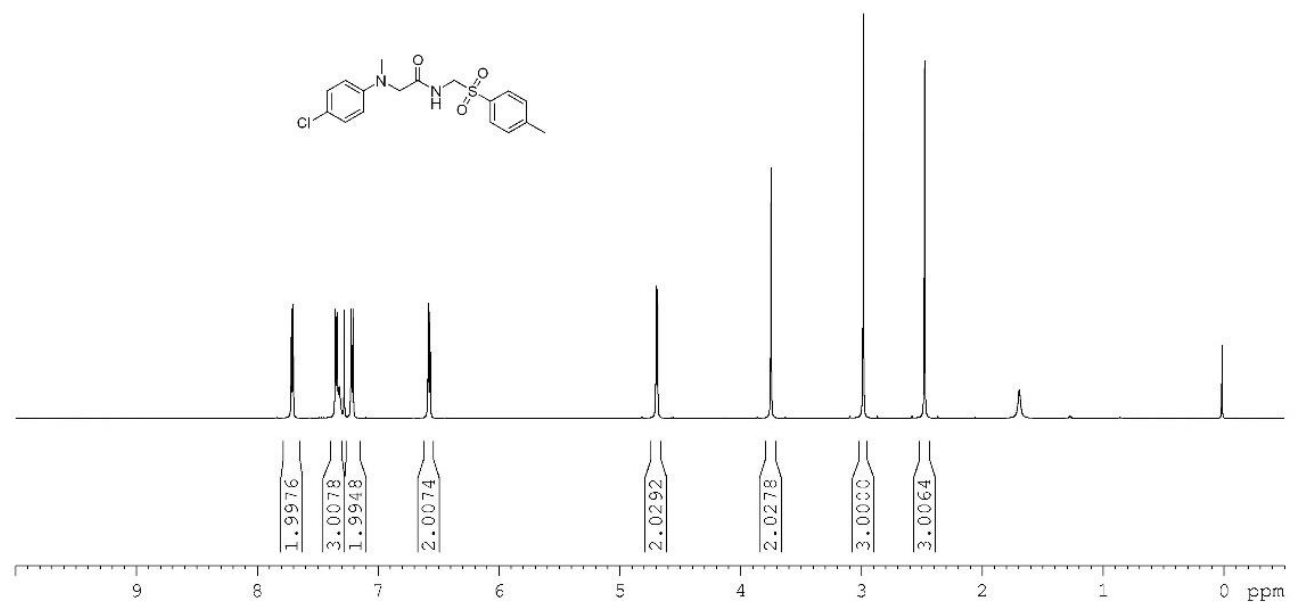


${ }^{13} \mathrm{C}\left\{{ }^{1} \mathrm{H}\right\}$ NMR (151 MHz, $\left.\mathrm{CDCl}_{3}\right)$ of compound $\mathbf{3 j}$

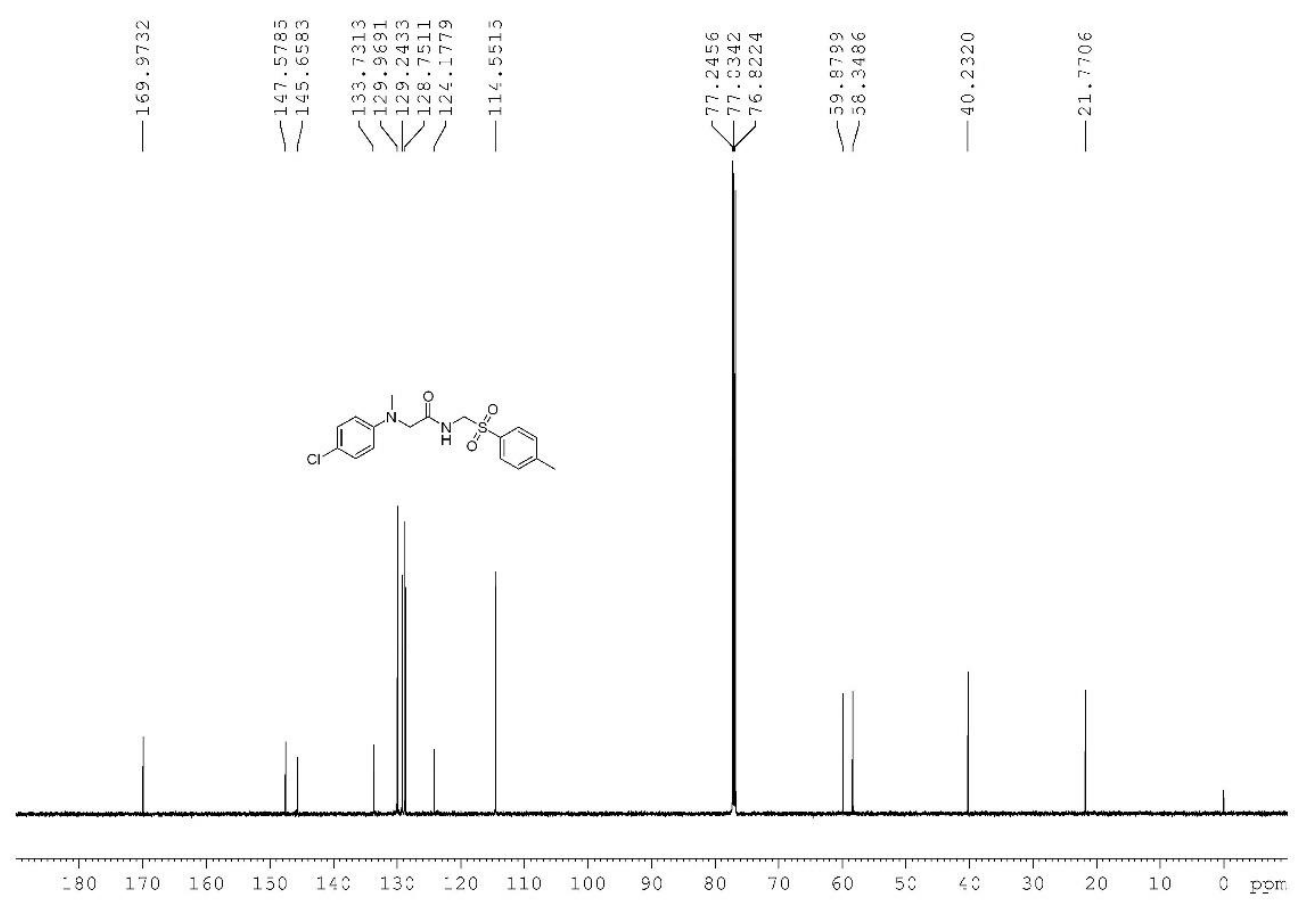

${ }^{1} \mathrm{H}$ NMR (600 MHz, $\mathrm{CDCl}_{3}$ ) of compound $\mathbf{3 k}$

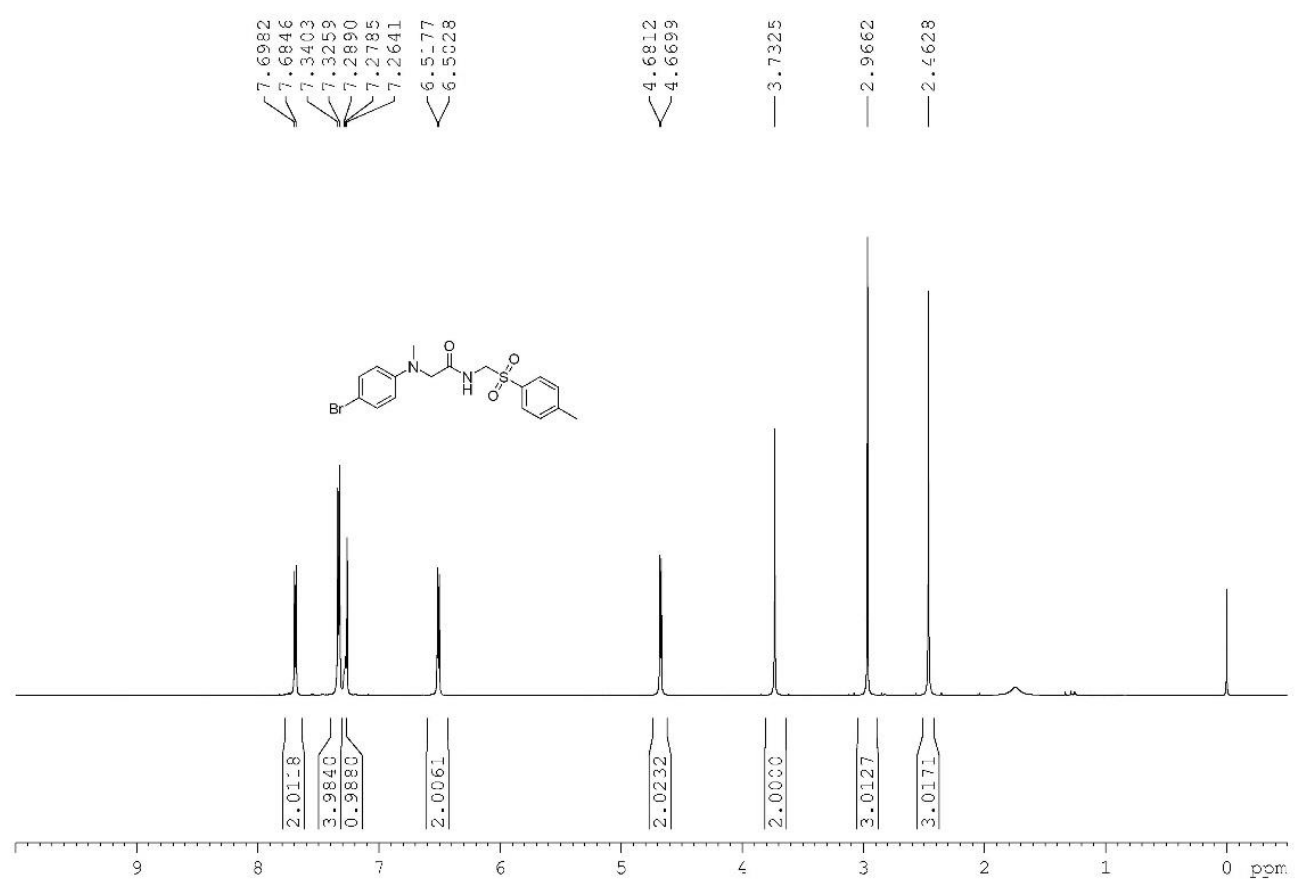


${ }^{13} \mathrm{C}\left\{{ }^{1} \mathrm{H}\right\}$ NMR (151 MHz, $\left.\mathrm{CDCl}_{3}\right)$ of compound 3k

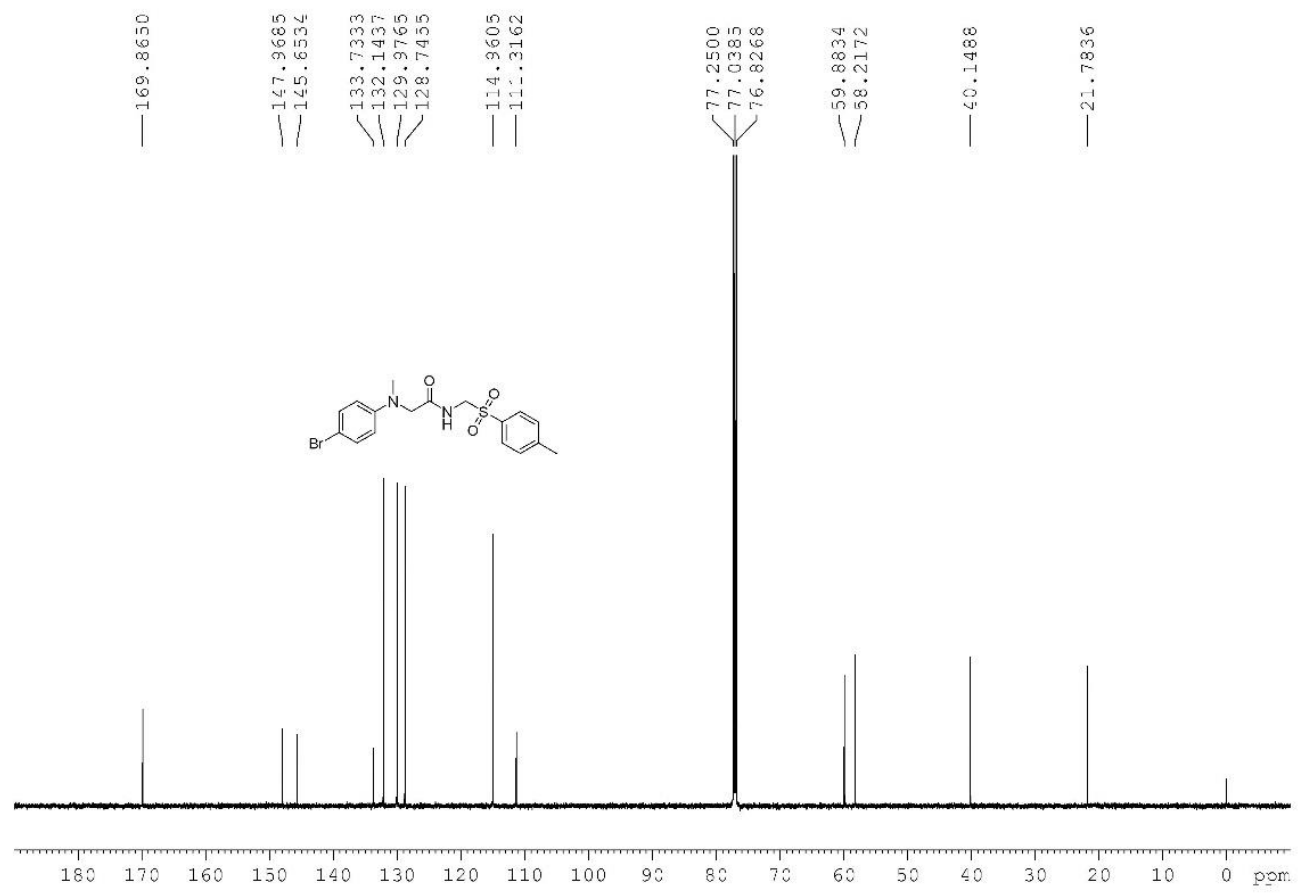

${ }^{1} \mathrm{H}$ NMR (600 MHz, $d_{6}$-DMSO) of compound $3 \mathrm{I}$

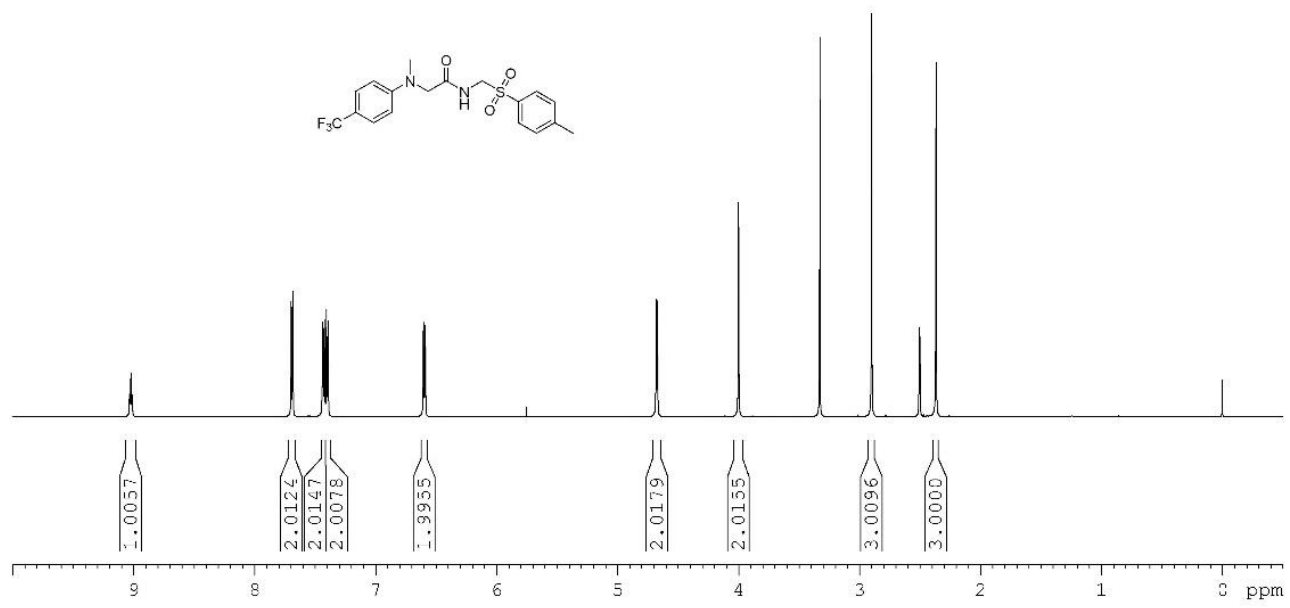


${ }^{13} \mathrm{C}\left\{{ }^{1} \mathrm{H}\right\}$ NMR (151 MHz, $d_{6}$-DMSO) of compound 3I

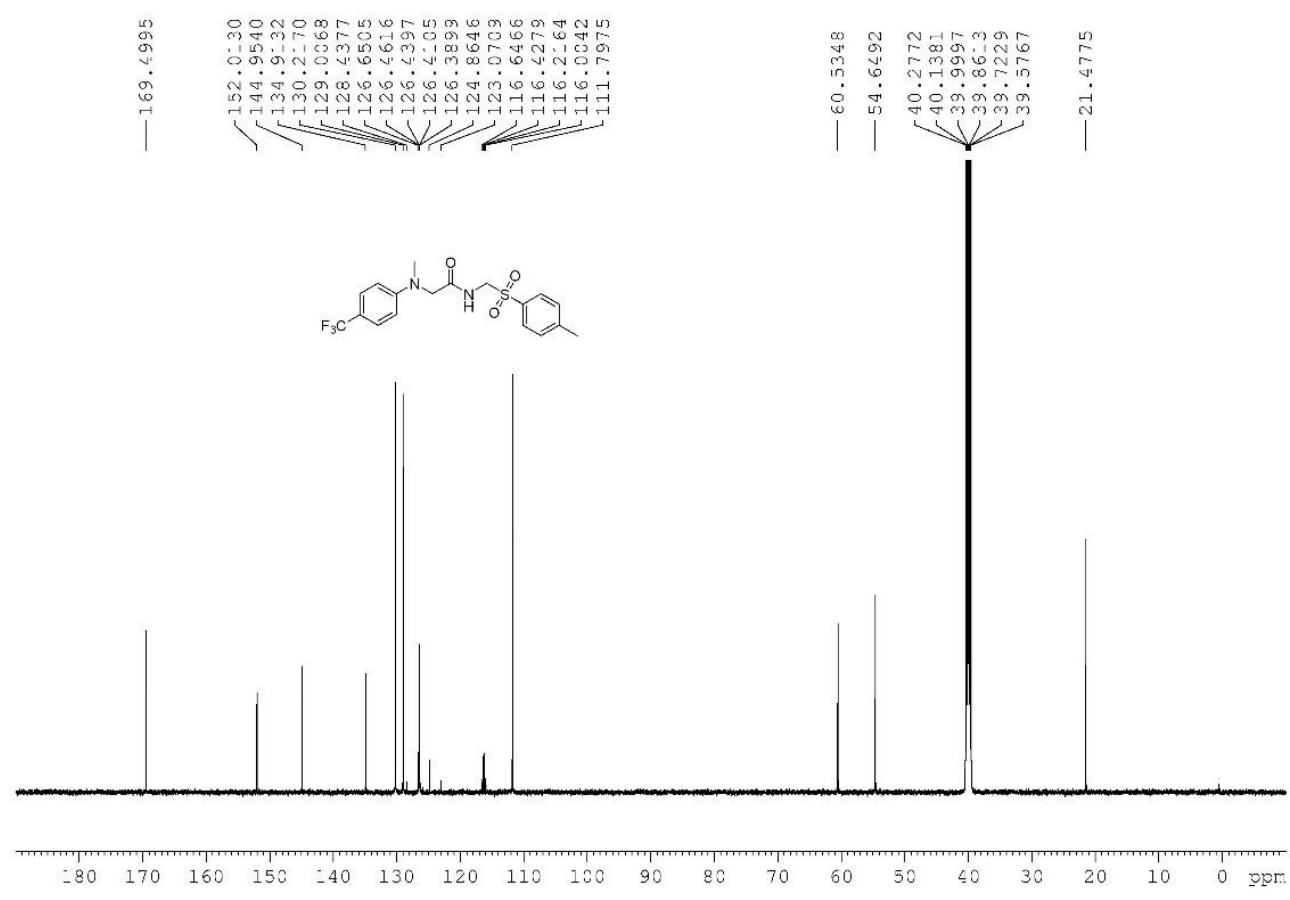

${ }^{19} \mathrm{~F}$ NMR (565 MHz, $d_{6}$-DMSO) of compound 31

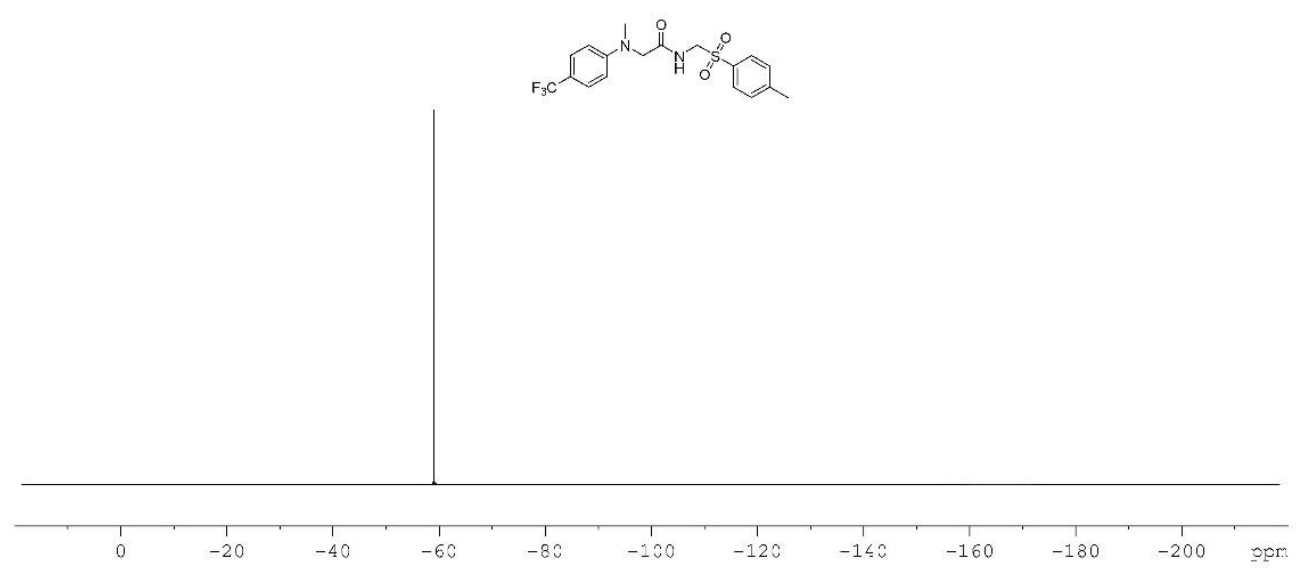


${ }^{1} \mathrm{H}$ NMR $\left(600 \mathrm{MHz}, \mathrm{CDCl}_{3}\right.$ ) of compound $\mathbf{3 m}$

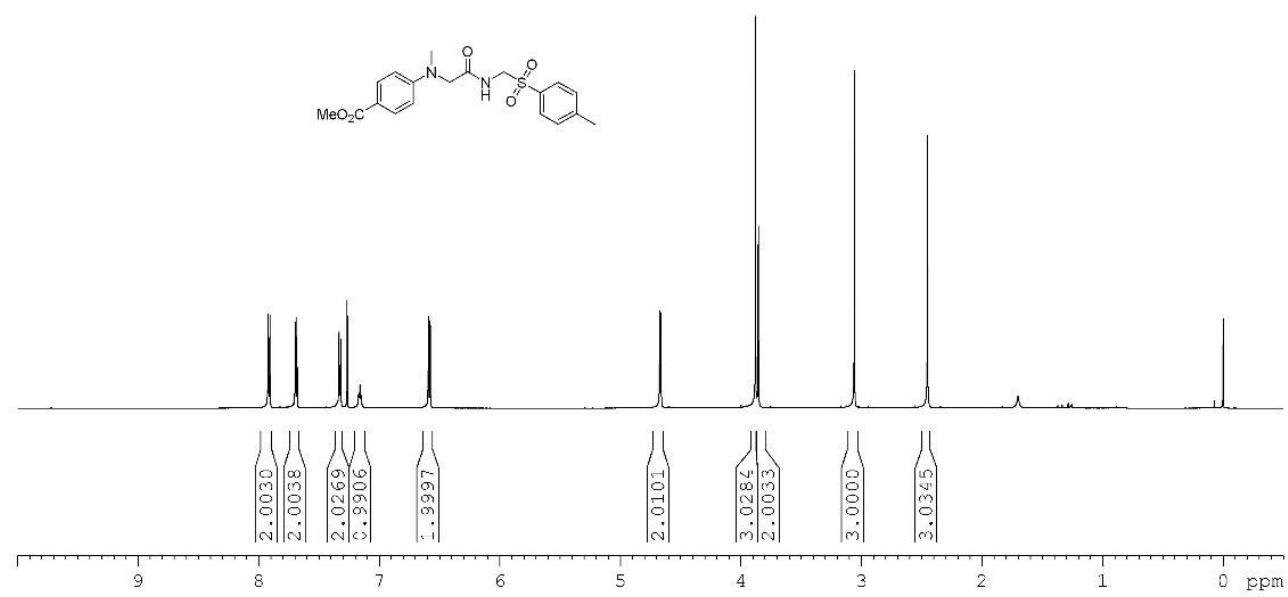

${ }^{13} \mathrm{C}\left\{{ }^{1} \mathrm{H}\right\}$ NMR (101 MHz, $\mathrm{CDCl}_{3}$ ) of compound $\mathbf{3 m}$
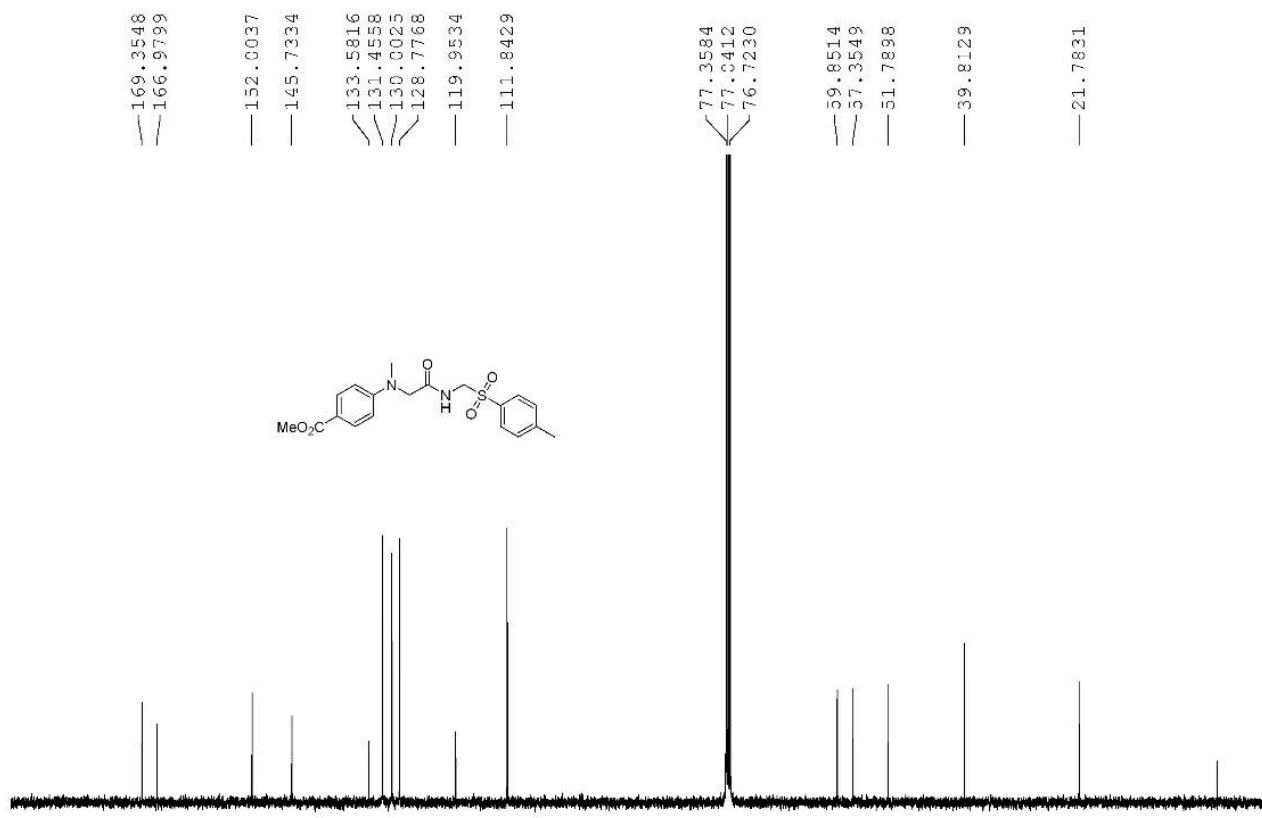

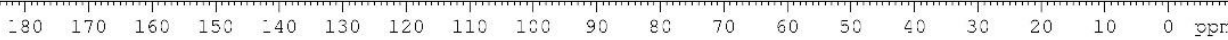


${ }^{1} \mathrm{H}$ NMR $\left(600 \mathrm{MHz}, \mathrm{CDCl}_{3}\right)$ of compound $\mathbf{3 n}$

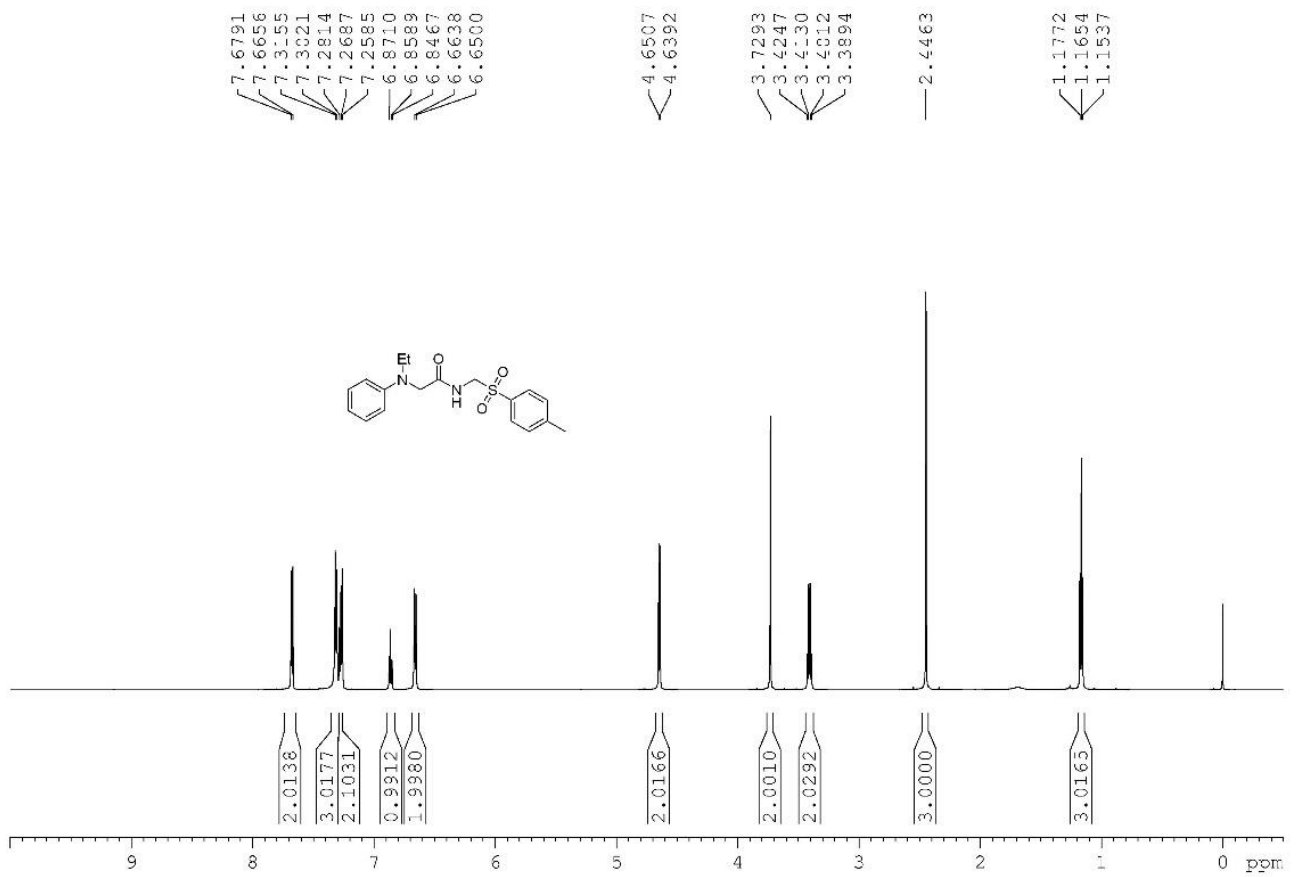

${ }^{13} \mathrm{C}\left\{{ }^{1} \mathrm{H}\right\}$ NMR (151 MHz, $\left.\mathrm{CDCl}_{3}\right)$ of compound $3 \mathbf{n}$
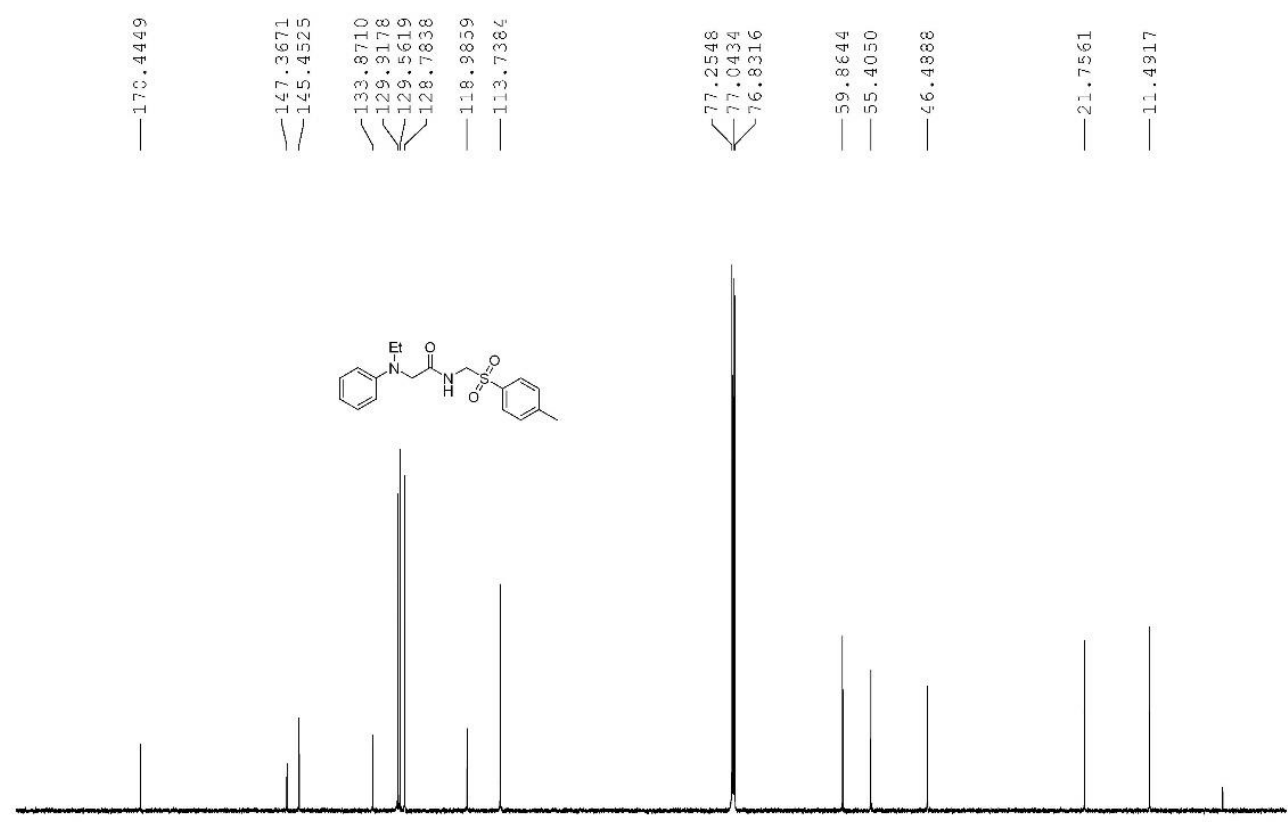

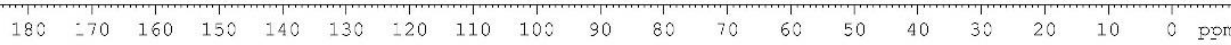


${ }^{1} \mathrm{H}$ NMR $\left(600 \mathrm{MHz}, \mathrm{CDCl}_{3}\right)$ of compound $3 \mathrm{o}$
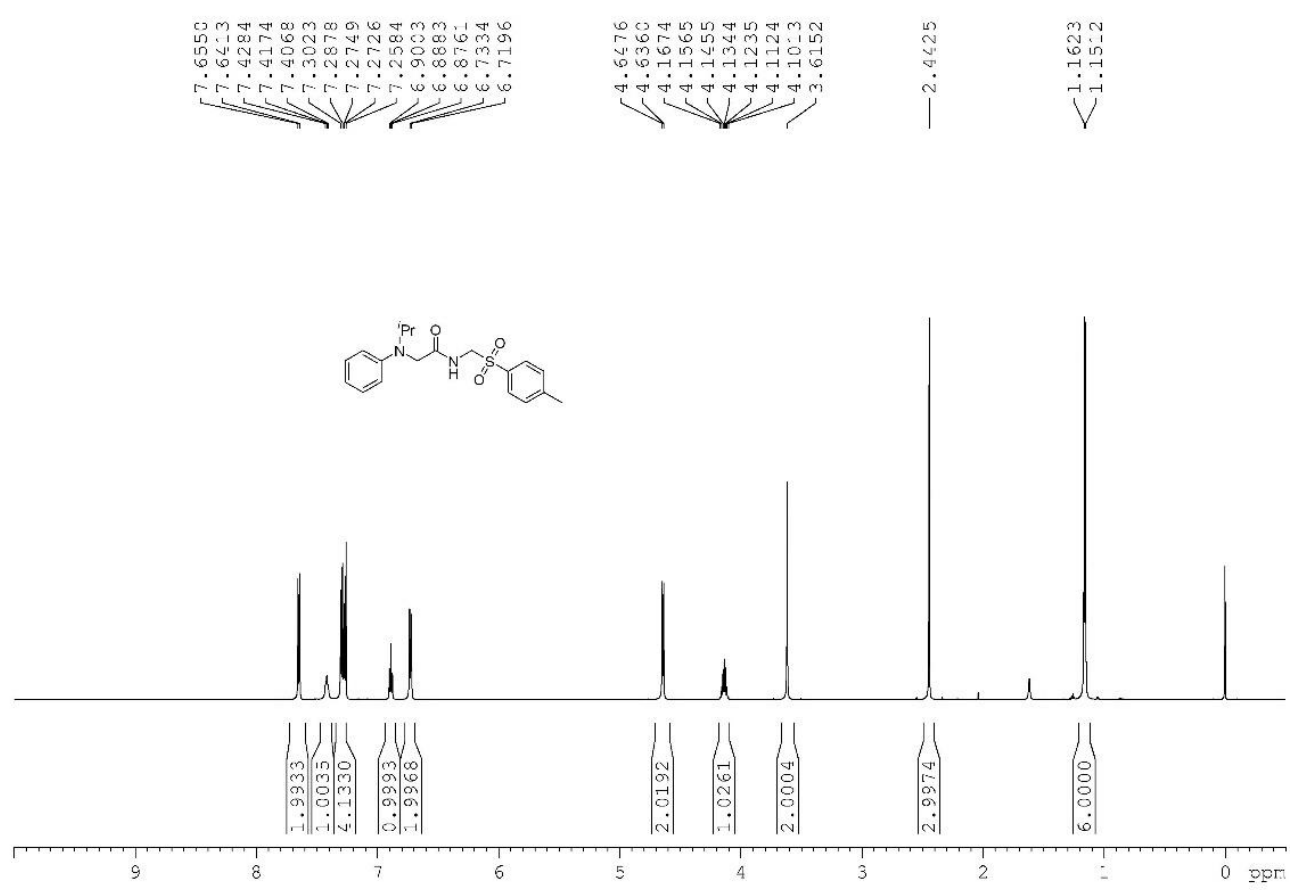

${ }^{13} \mathrm{C}\left\{{ }^{1} \mathrm{H}\right\}$ NMR (101 MHz, $\left.\mathrm{CDCl}_{3}\right)$ of compound 3o

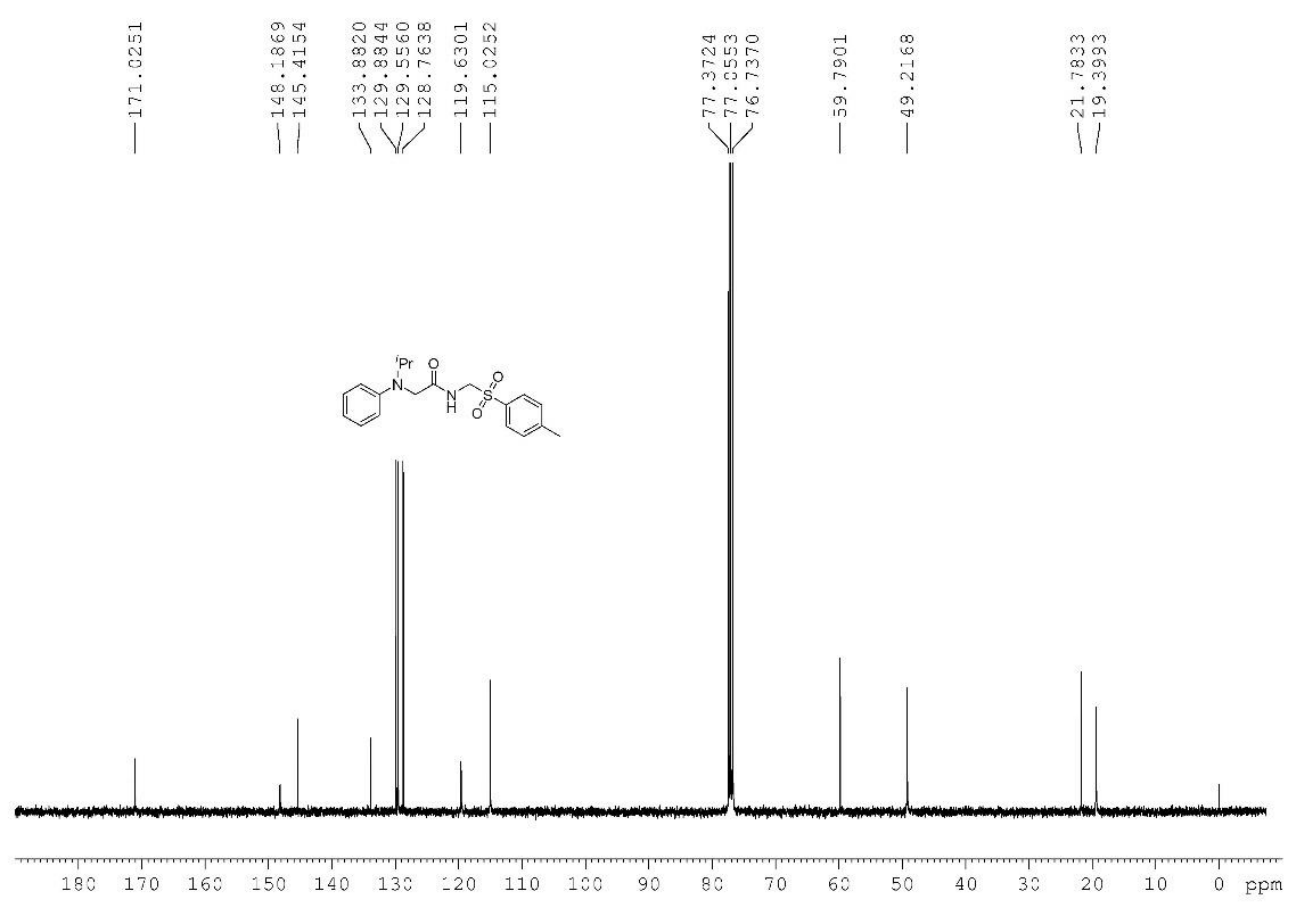


${ }^{1} \mathrm{H}$ NMR $\left(600 \mathrm{MHz}, \mathrm{CDCl}_{3}\right.$ ) of compound $\mathbf{3 p}$
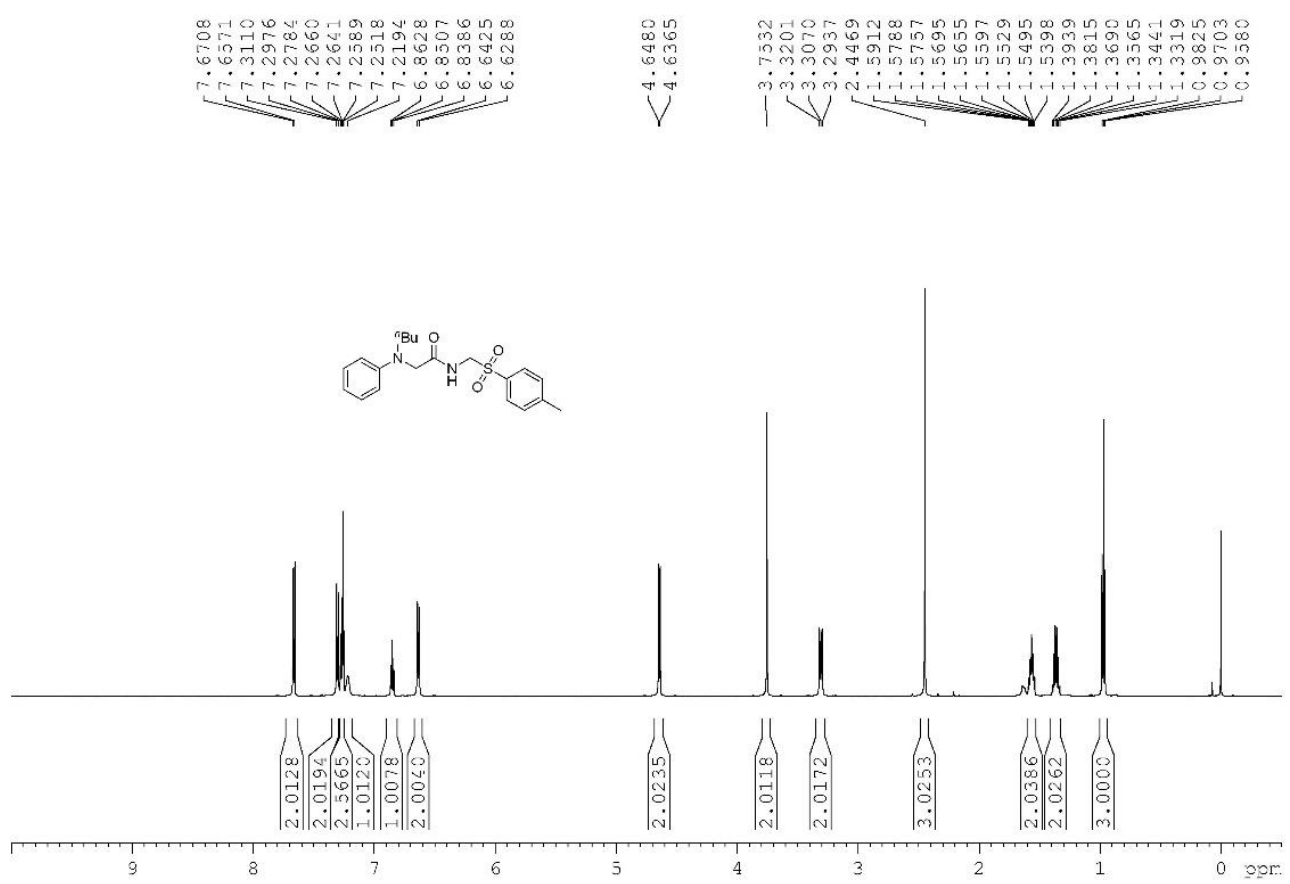

${ }^{13} \mathrm{C}\left\{{ }^{1} \mathrm{H}\right\}$ NMR (101 MHz, $\left.\mathrm{CDCl}_{3}\right)$ of compound 3p

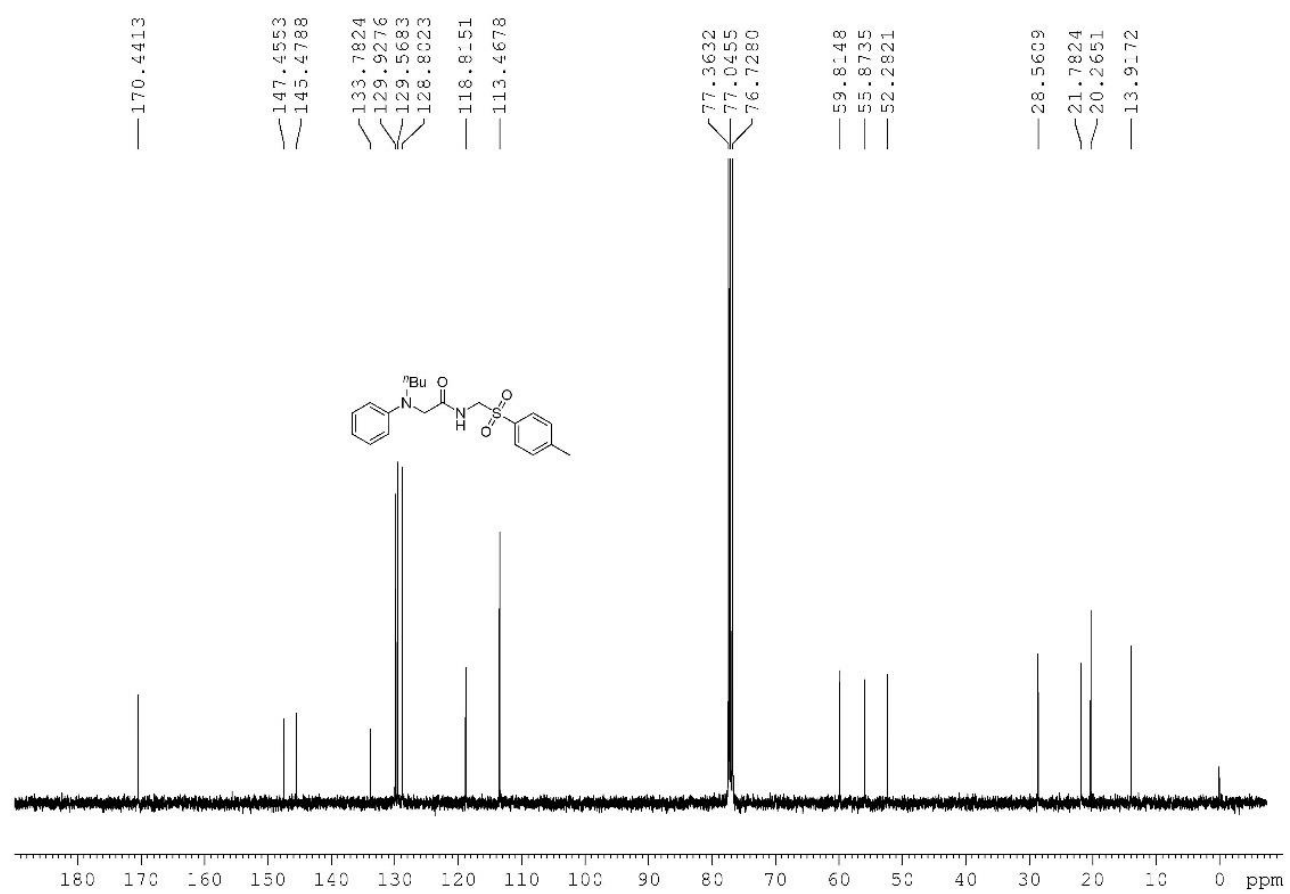


${ }^{1} \mathrm{H}$ NMR (600 MHz, $\mathrm{CDCl}_{3}$ ) of compound $\mathbf{3 q}$
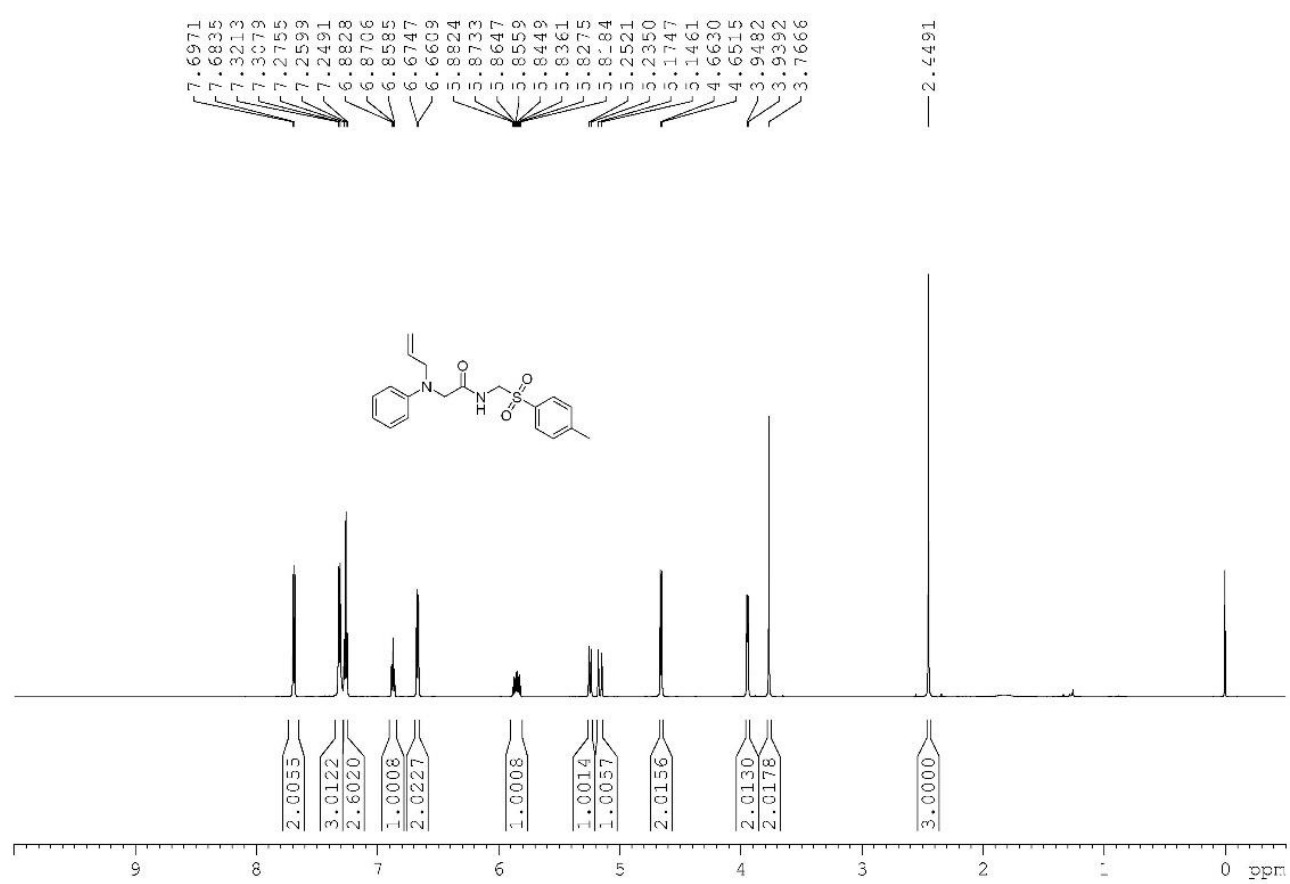

${ }^{13} \mathrm{C}\left\{{ }^{1} \mathrm{H}\right\}$ NMR (151 MHz, $\left.\mathrm{CDCl}_{3}\right)$ of compound $\mathbf{3 q}$

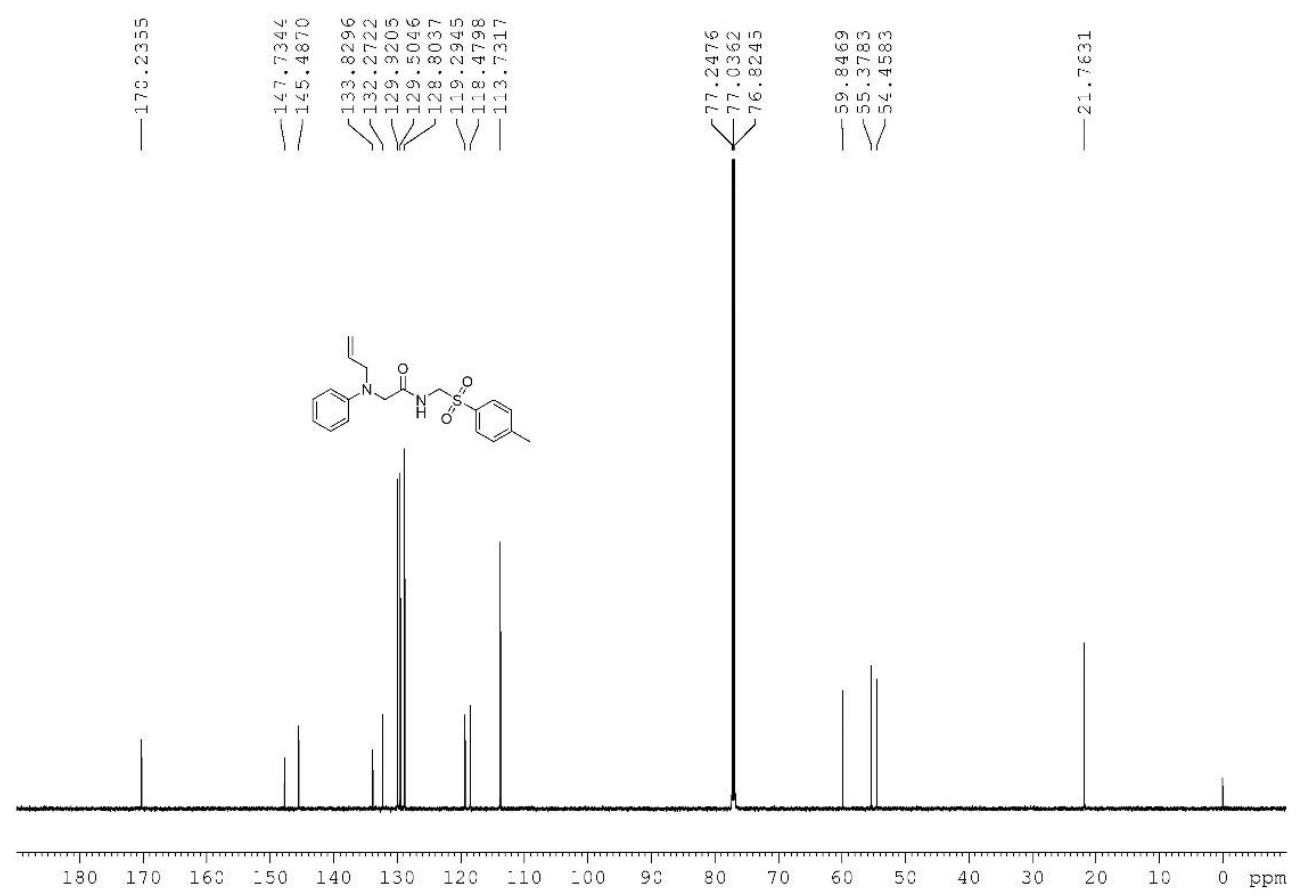


${ }^{1} \mathrm{H}$ NMR $\left(600 \mathrm{MHz}, \mathrm{CDCl}_{3}\right)$ of compound $\mathbf{3 r}$

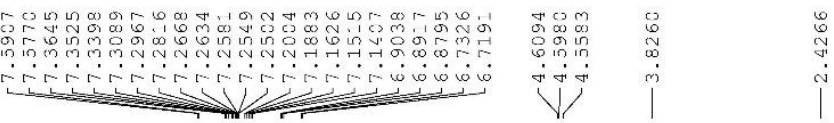

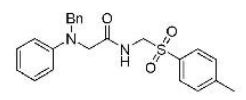

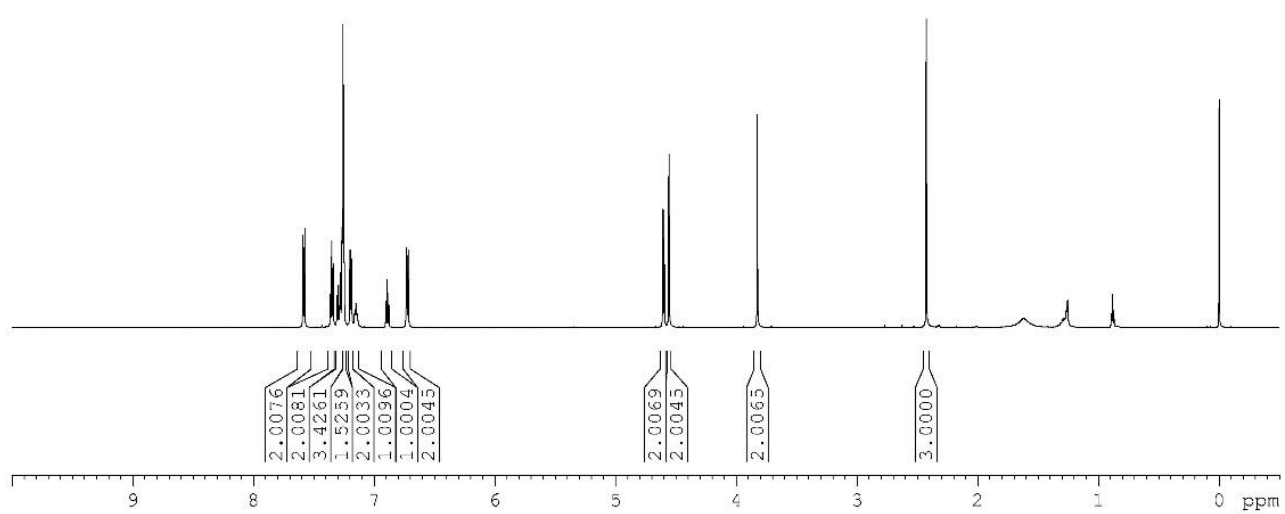

${ }^{13} \mathrm{C}\left\{{ }^{1} \mathrm{H}\right\}$ NMR (101 MHz, $\left.\mathrm{CDCl}_{3}\right)$ of compound $\mathbf{3 r}$

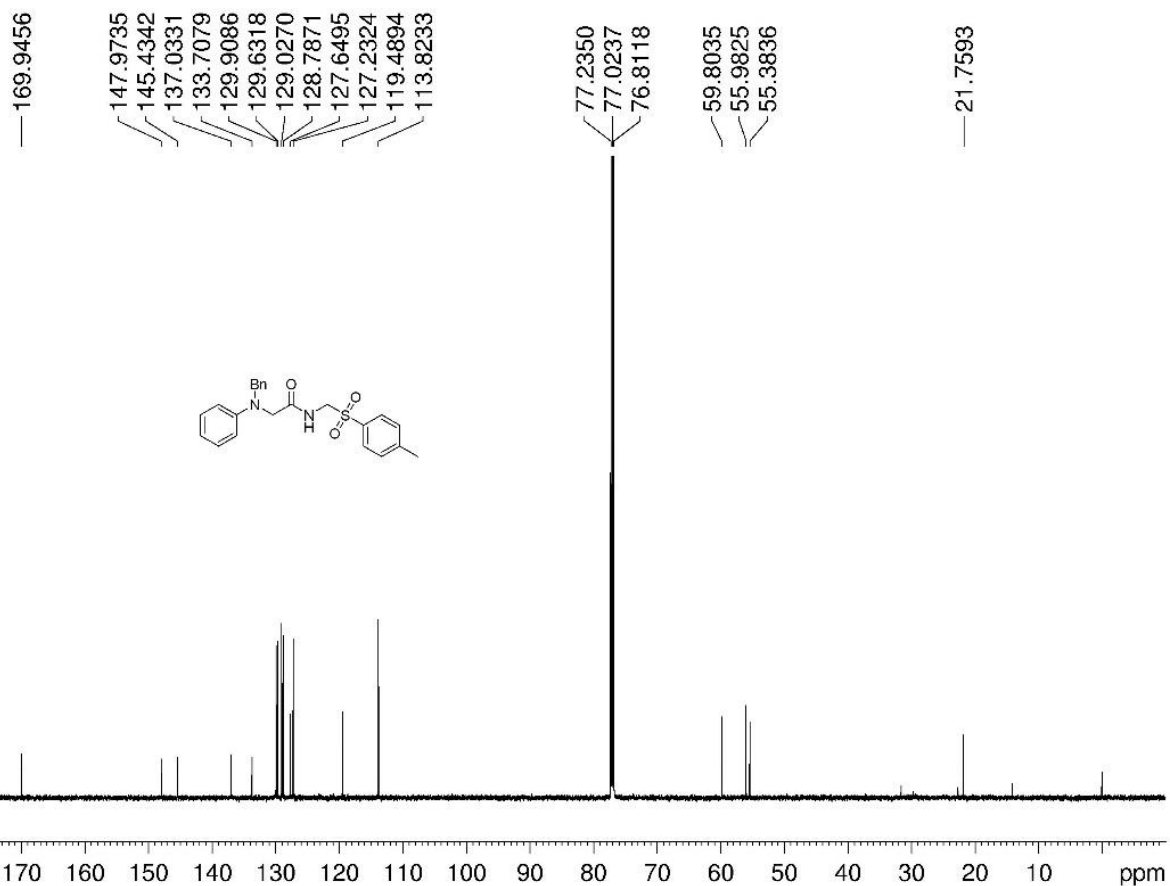


${ }^{1} \mathrm{H}$ NMR (600 MHz, $\left.\mathrm{CDCl}_{3}\right)$ of compound $\mathbf{3 s}$

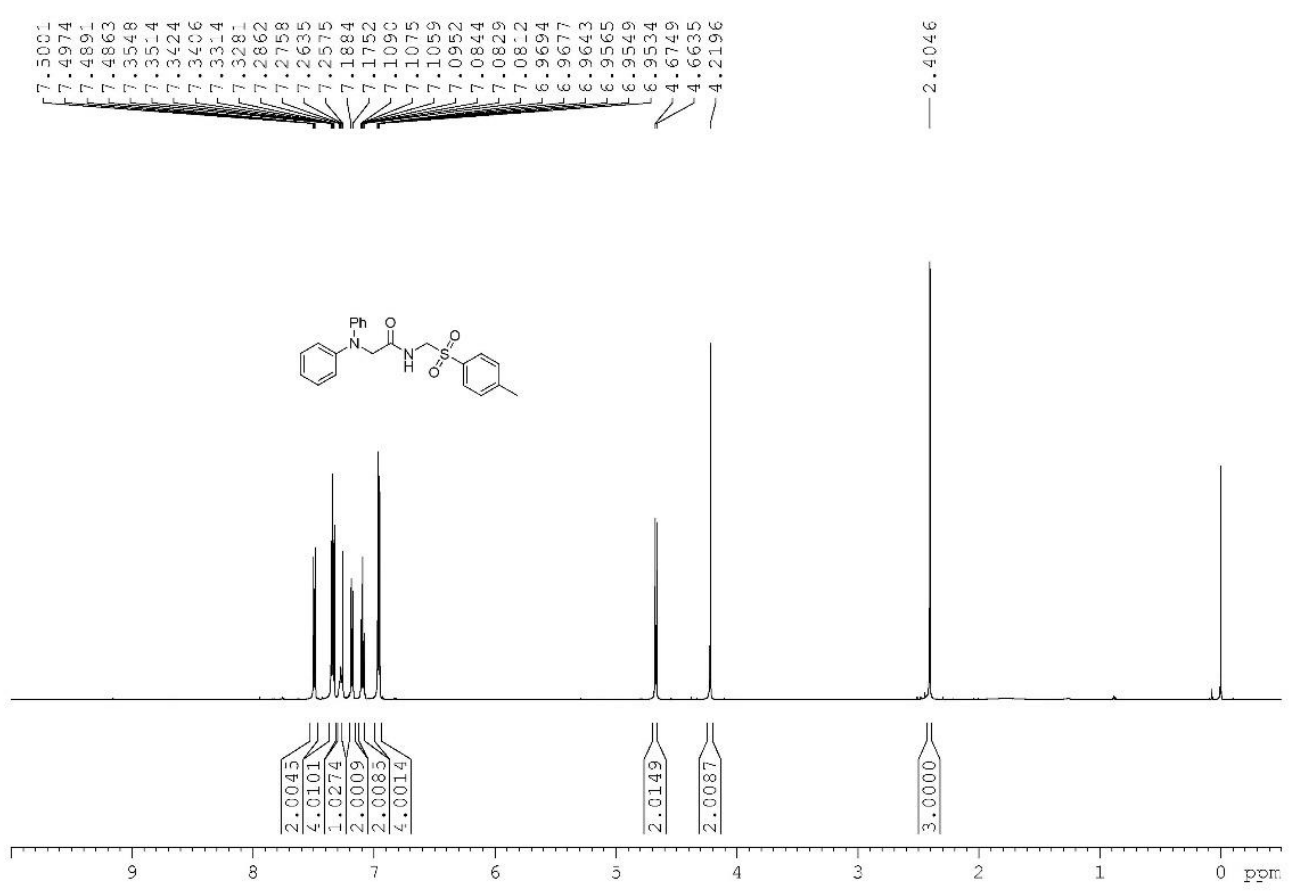

${ }^{13} \mathrm{C}\left\{{ }^{1} \mathrm{H}\right\}$ NMR (151 MHz, $\left.\mathrm{CDCl}_{3}\right)$ of compound $\mathbf{3 s}$

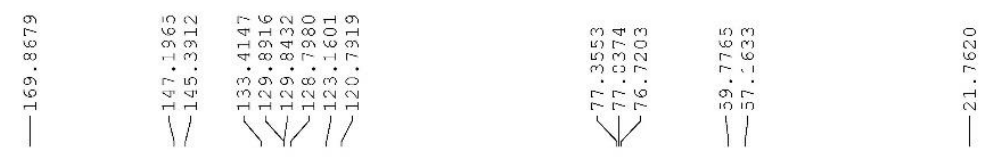

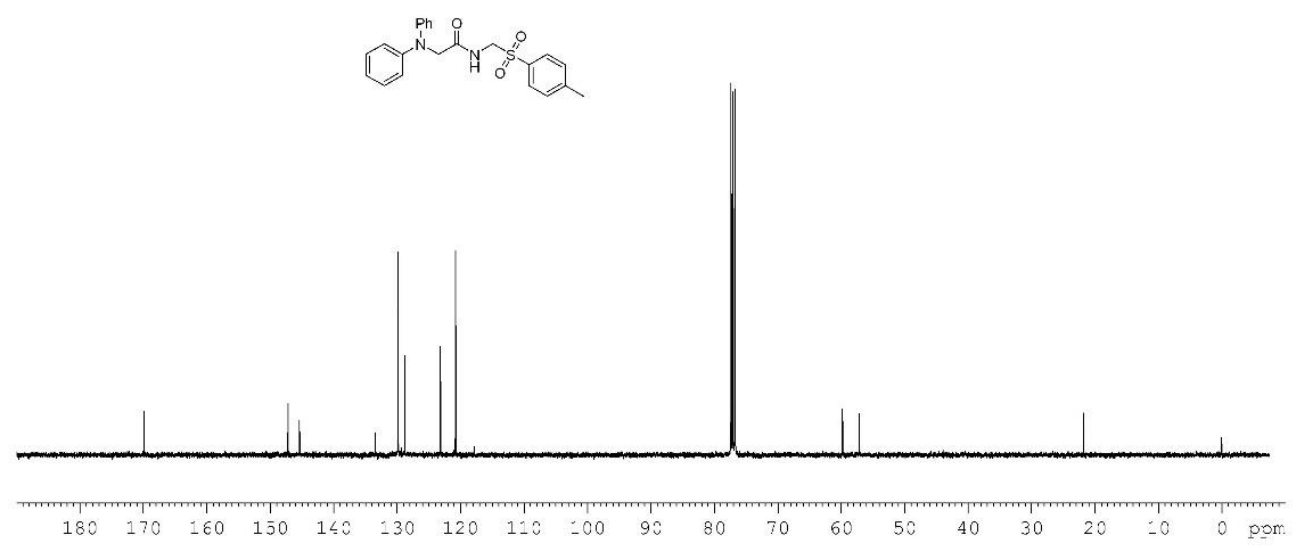


${ }^{1} \mathrm{H}$ NMR (600 MHz, $d_{6}$-DMSO) of compound $\mathbf{3 t}$

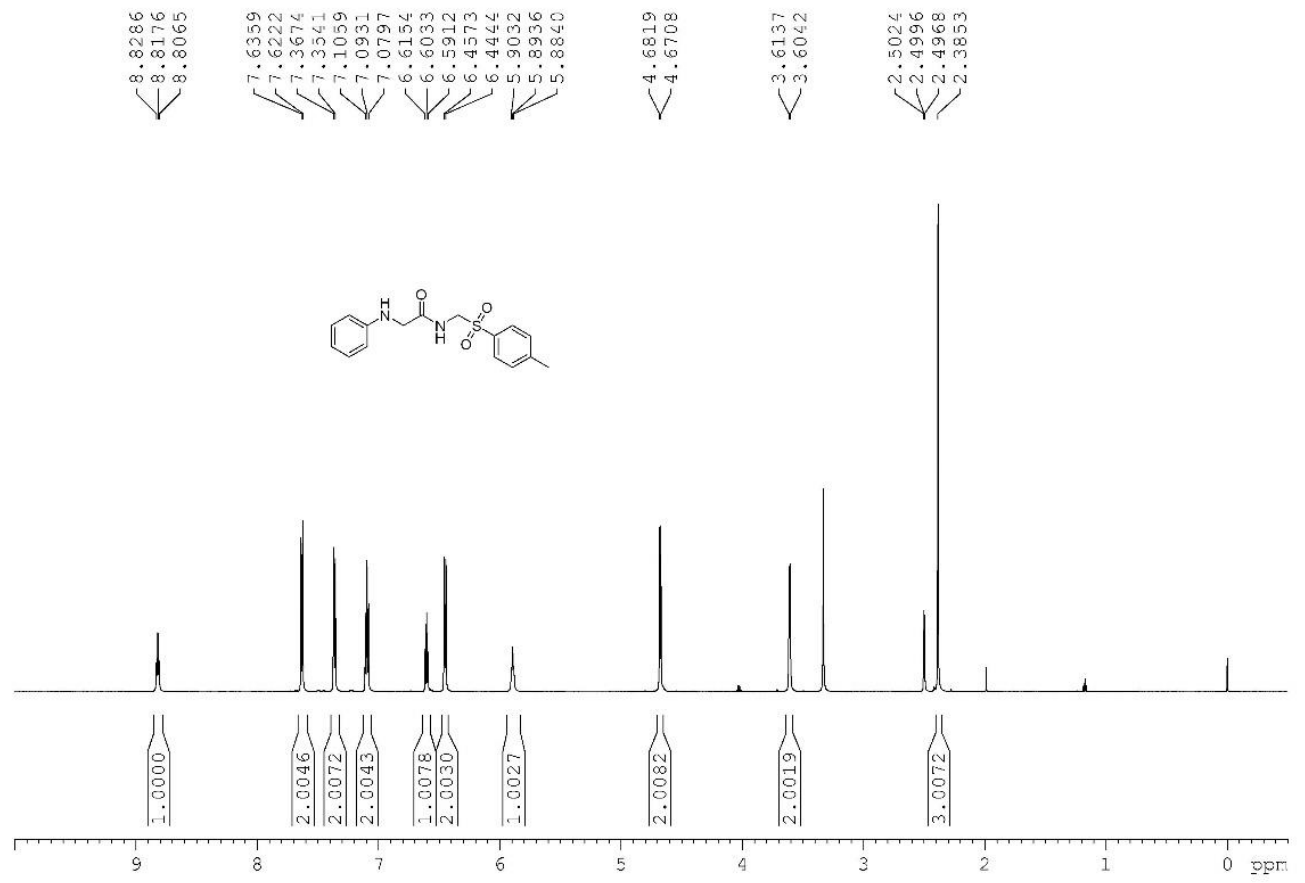

${ }^{13} \mathrm{C}\left\{{ }^{1} \mathrm{H}\right\}$ NMR (151 MHz, $d_{6}$-DMSO) of compound $\mathbf{3 t}$
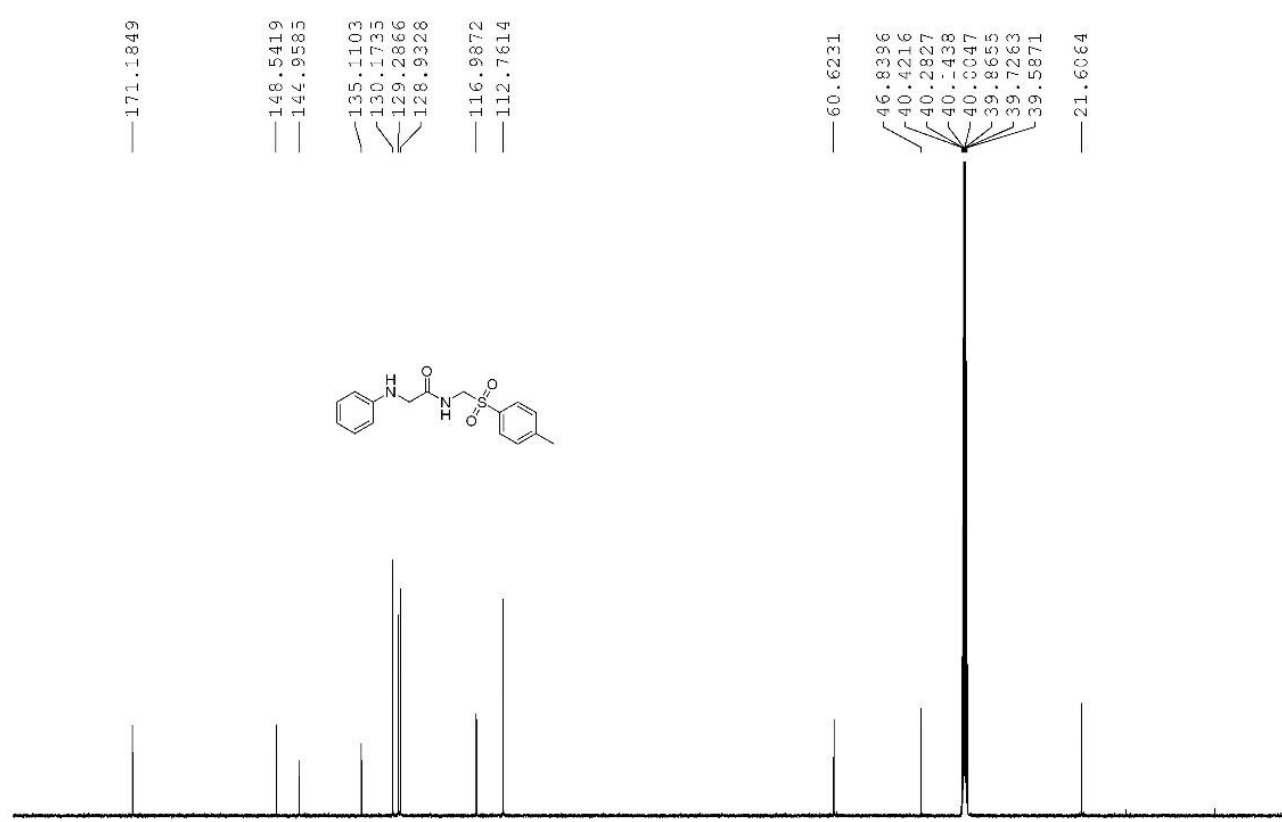

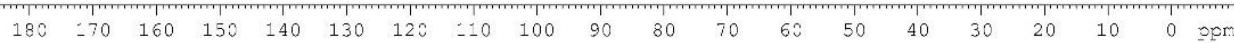


${ }^{1} \mathrm{H}$ NMR (600 MHz, $d_{6}$-DMSO) of compound $\mathbf{3 u}$
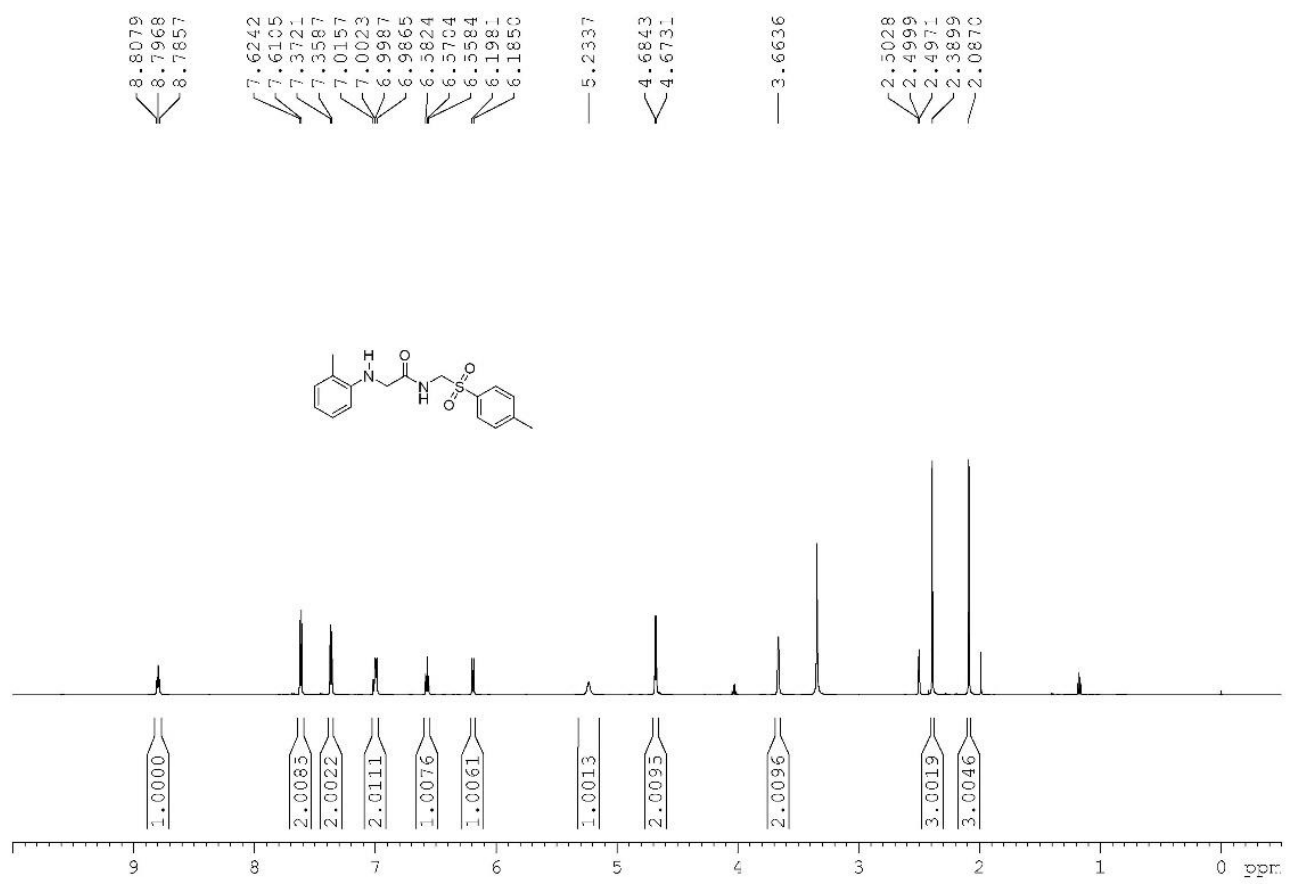

${ }^{13} \mathrm{C}\left\{{ }^{1} \mathrm{H}\right\}$ NMR (151 MHz, $d_{6}$-DMSO) of compound $\mathbf{3 u}$
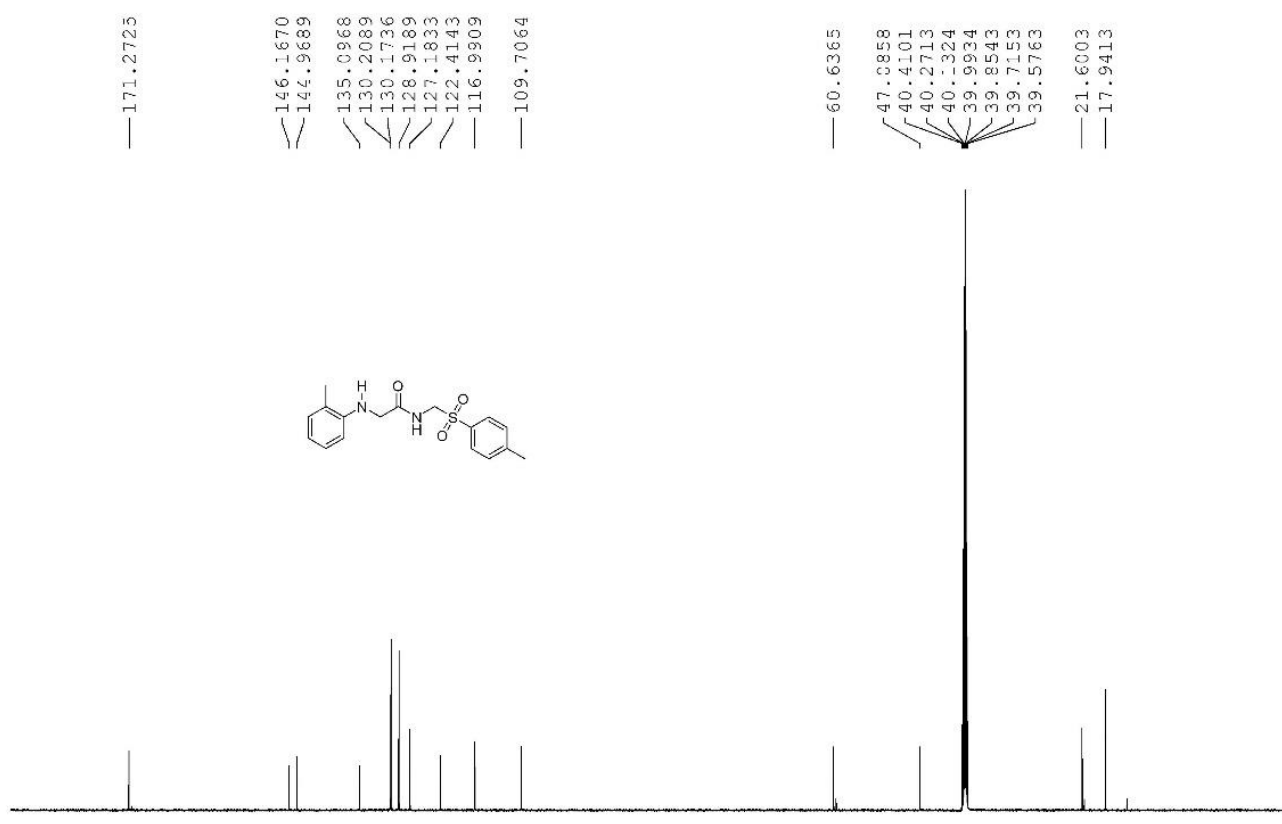

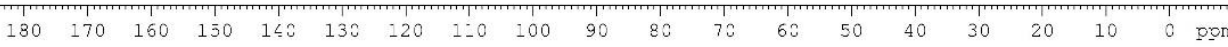


${ }^{1} \mathrm{H}$ NMR (600 MHz, $d_{6}$-DMSO) of compound $\mathbf{3 v}$
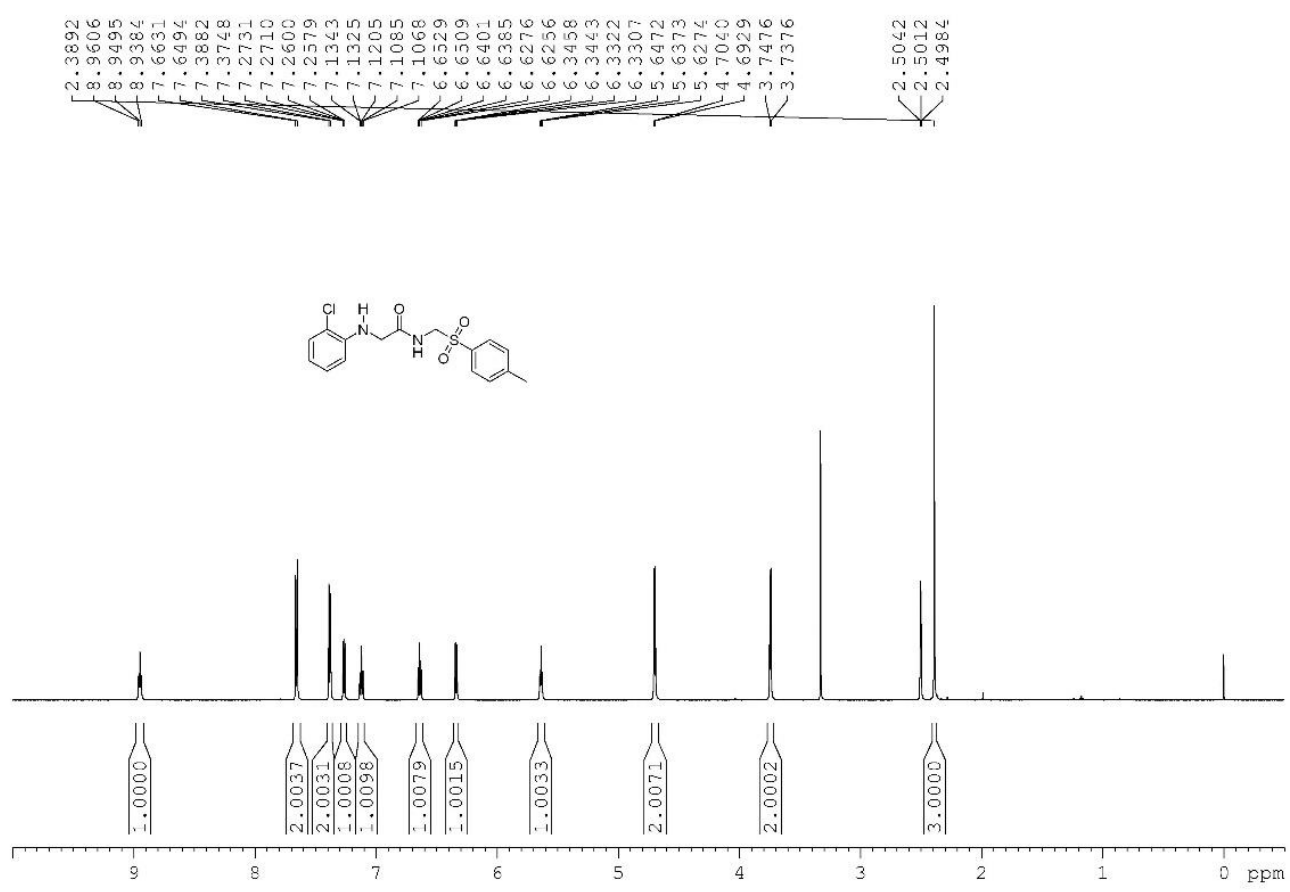

${ }^{13} \mathrm{C}\left\{{ }^{1} \mathrm{H}\right\}$ NMR (151 MHz, $d_{6}$-DMSO) of compound $\mathbf{3 v}$
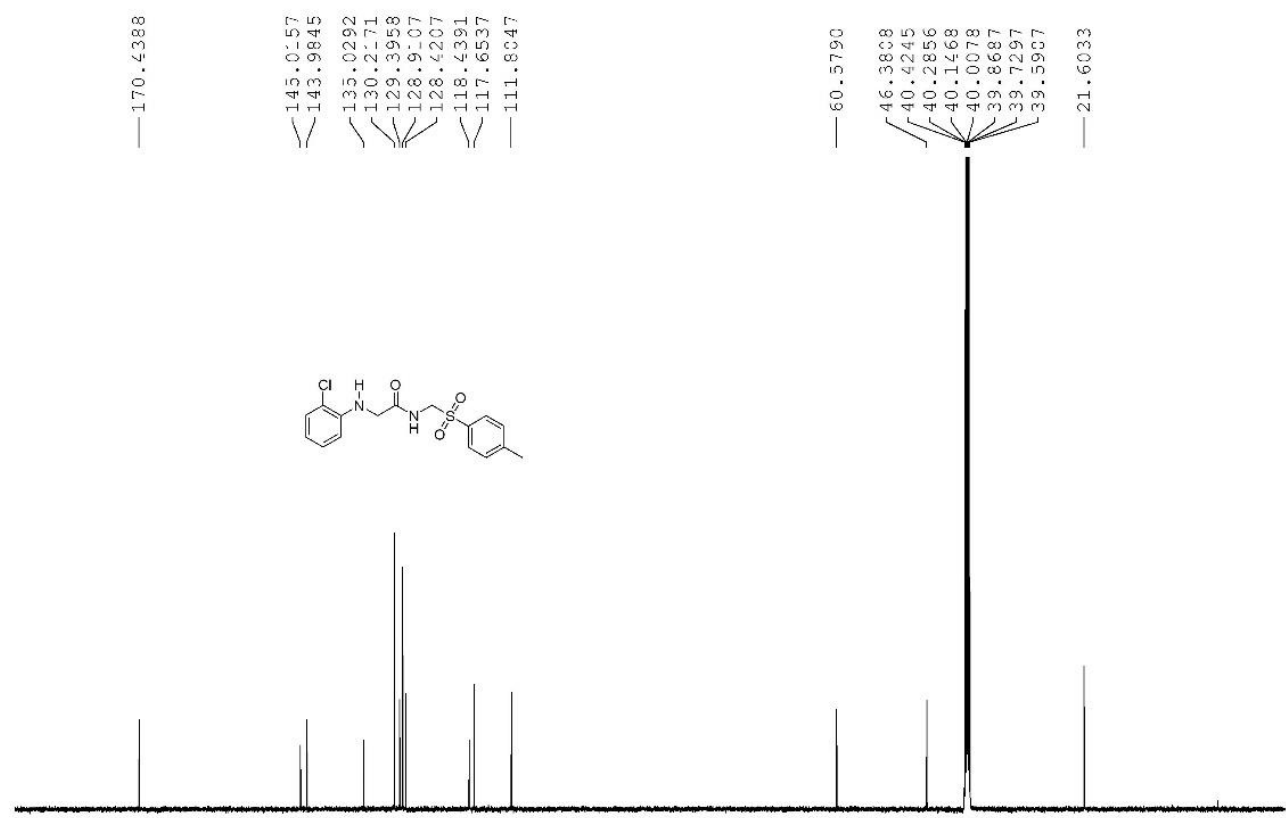

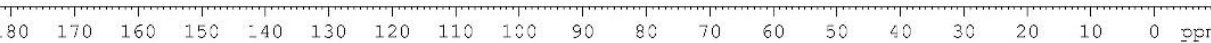


${ }^{1} \mathrm{H}$ NMR (600 MHz, $d_{6}$-DMSO) of compound 3w

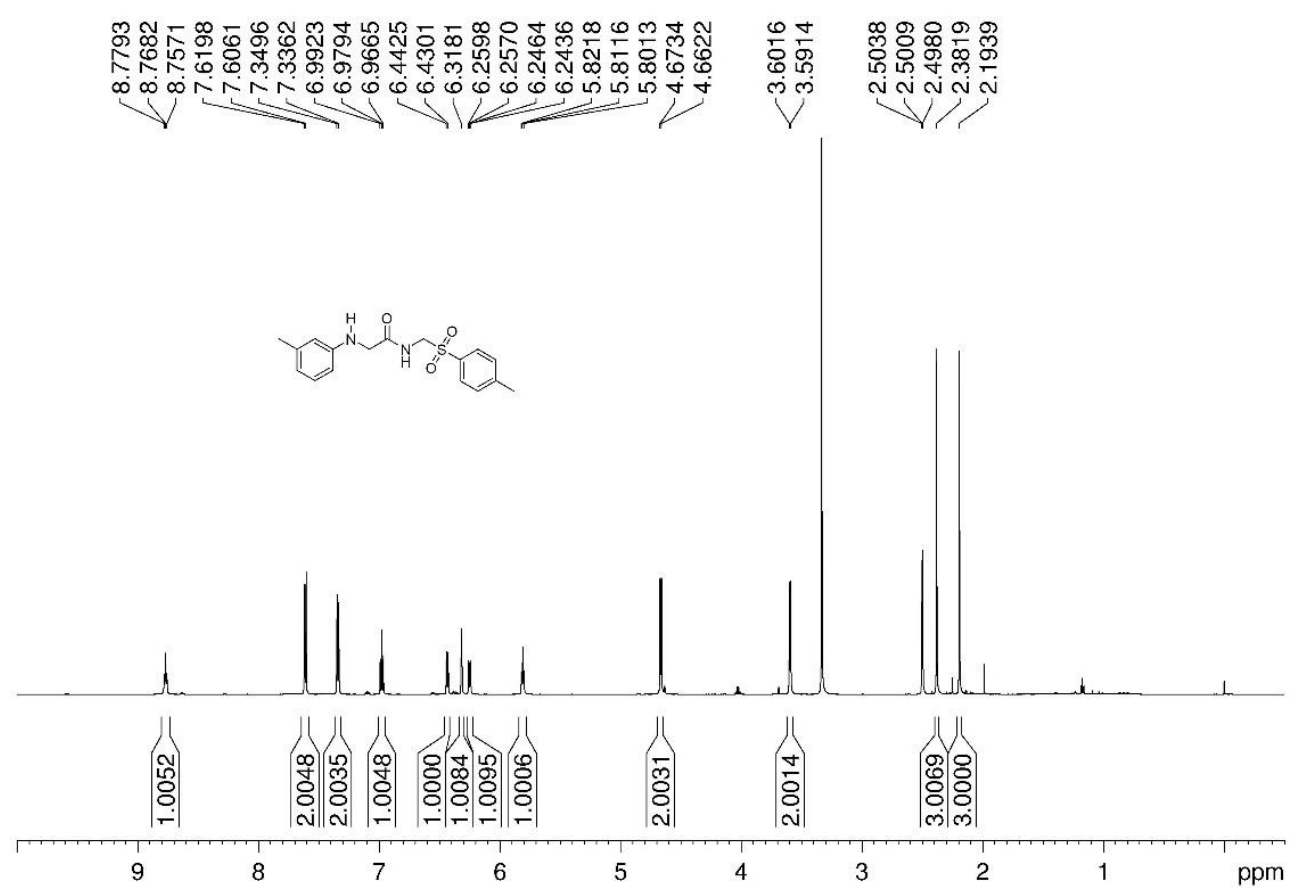

${ }^{13} \mathrm{C}\left\{{ }^{1} \mathrm{H}\right\}$ NMR (151 MHz, $d_{6}$-DMSO) of compound $\mathbf{3 w}$
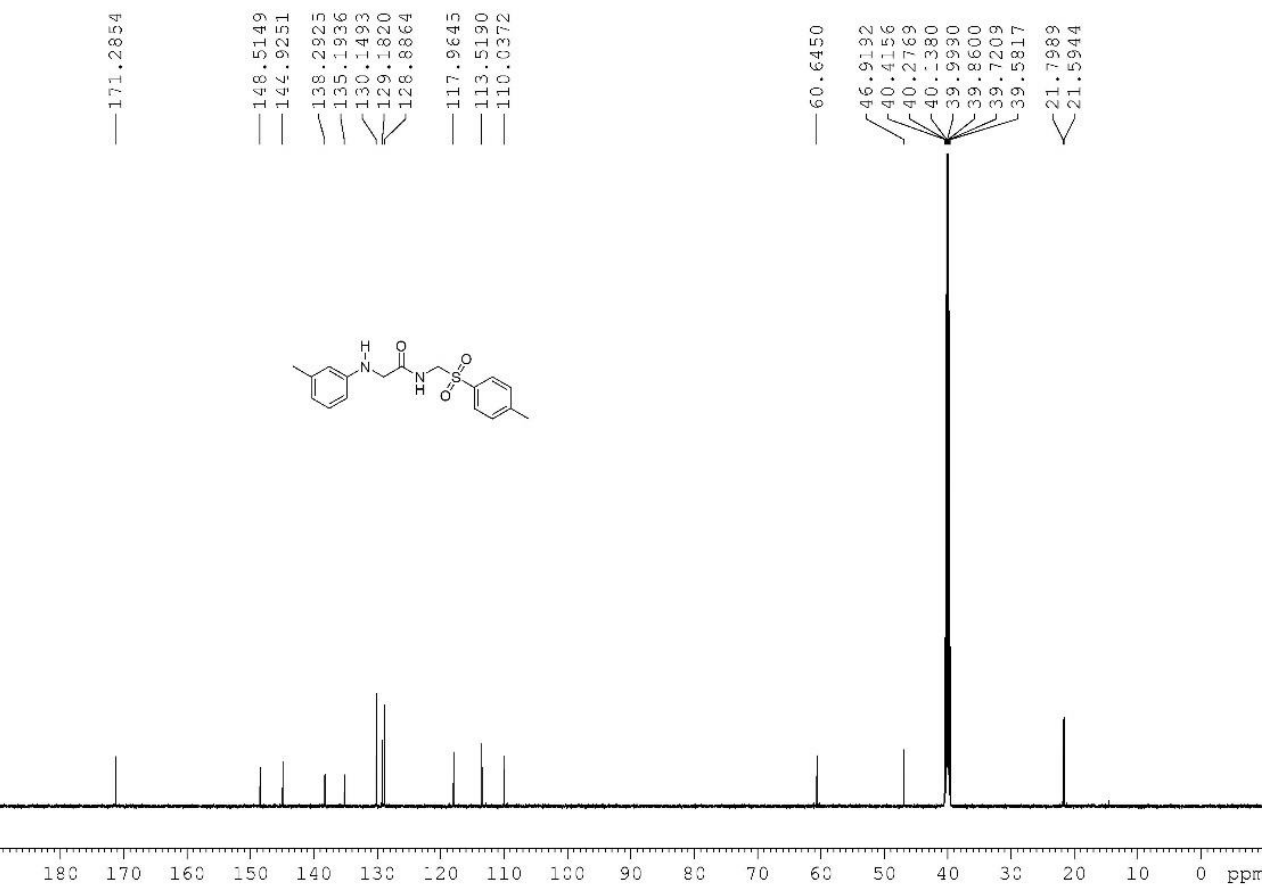
${ }^{1} \mathrm{H}$ NMR (600 MHz, $d_{6}$-DMSO) of compound $\mathbf{3 x}$
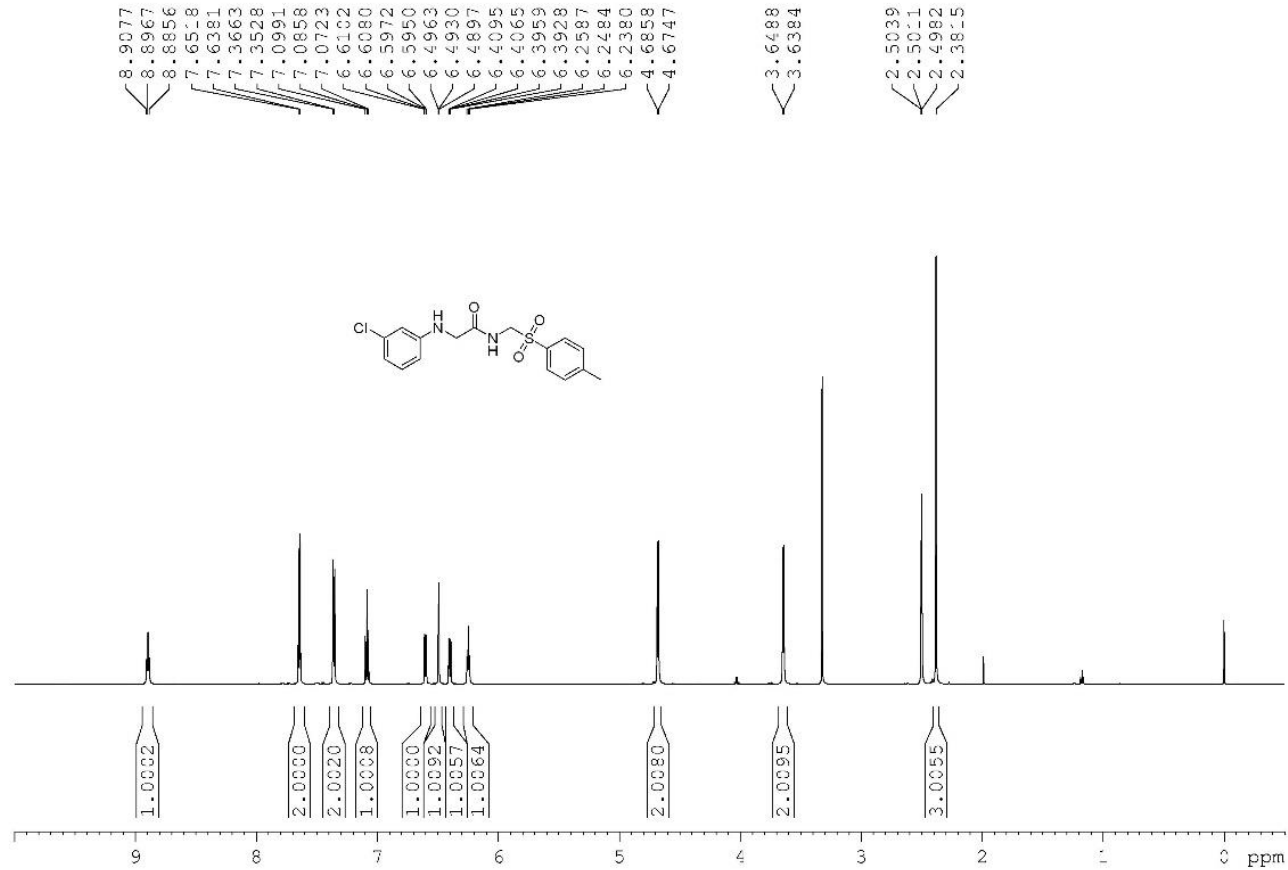

${ }^{13} \mathrm{C}\left\{{ }^{1} \mathrm{H}\right\}$ NMR (151 MHz, $d_{6}$-DMSO) of compound $\mathbf{3 x}$
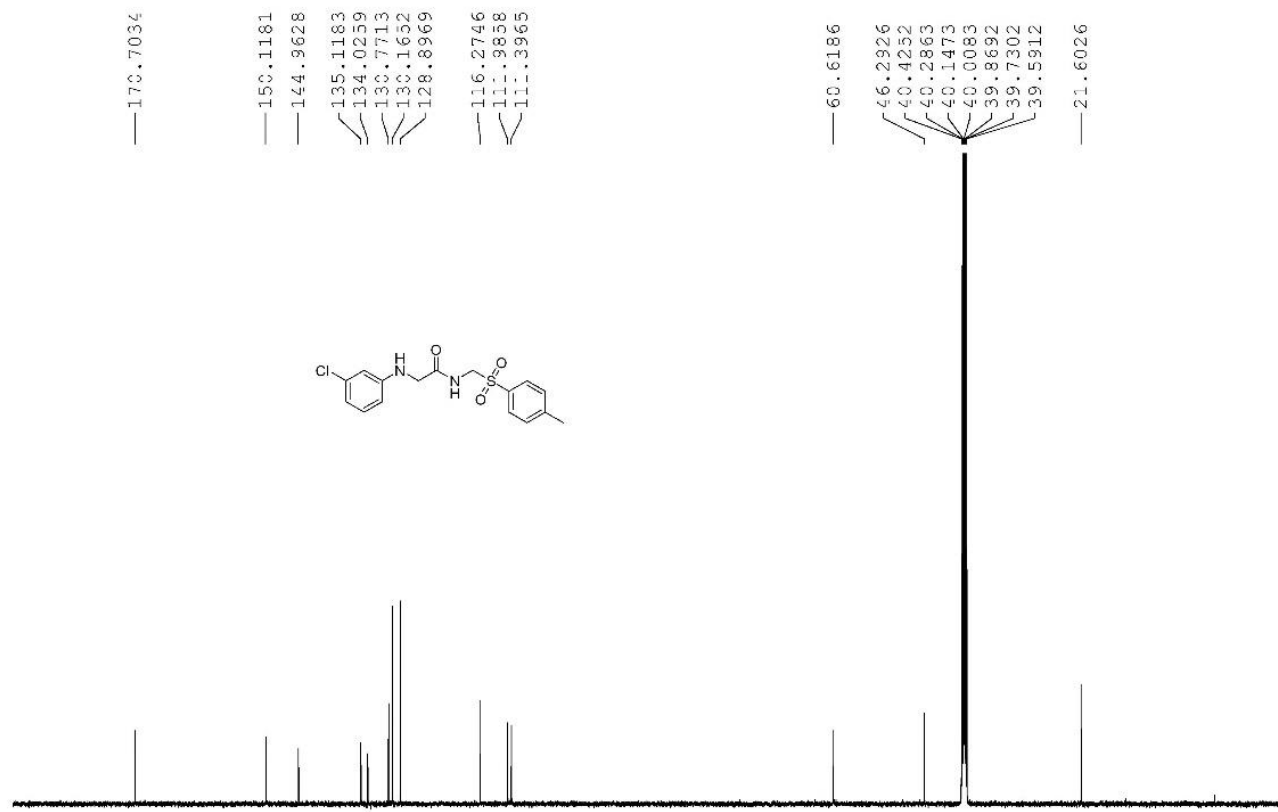

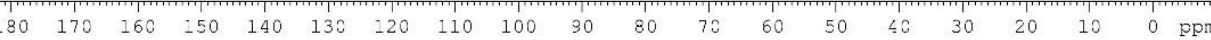


${ }^{1} \mathrm{H}$ NMR (600 MHz, $d_{6}$-DMSO) of compound $\mathbf{3 y}$
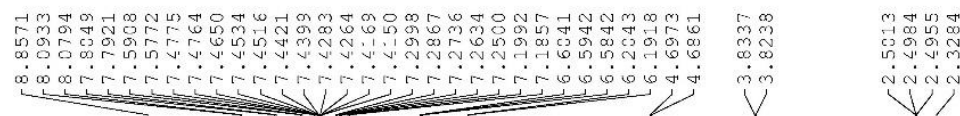

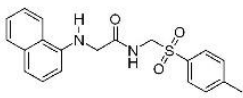

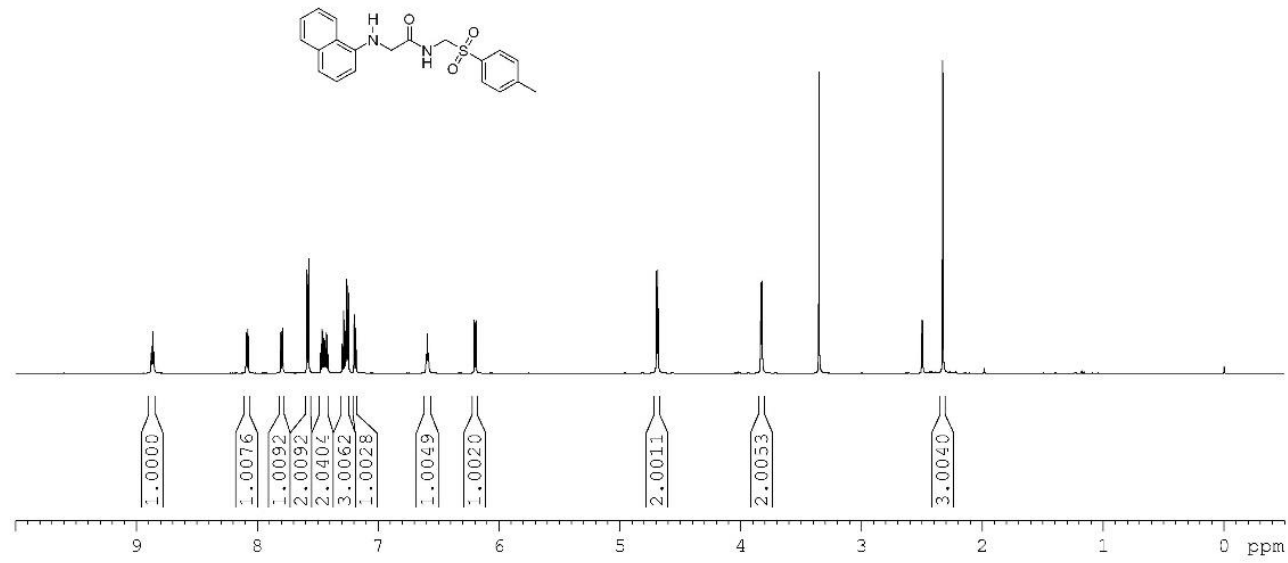

${ }^{13} \mathrm{C}\left\{{ }^{1} \mathrm{H}\right\}$ NMR (151 MHz, $d_{6}$-DMSO) of compound $\mathbf{3 y}$
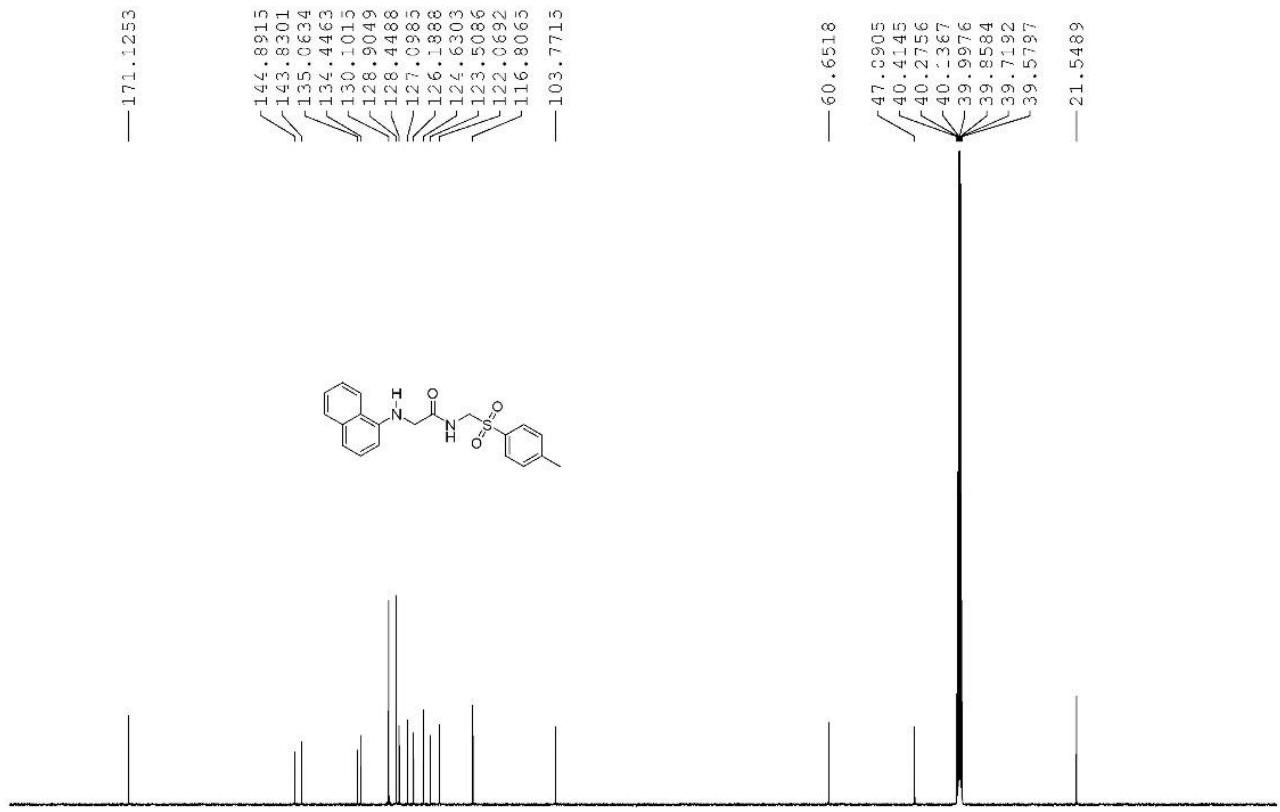

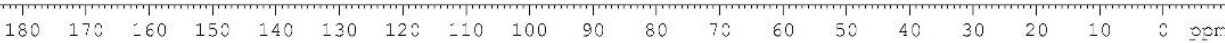


${ }^{1} \mathrm{H}$ NMR (600 MHz, $d_{6}$-DMSO) of compound $\mathbf{3 z}$
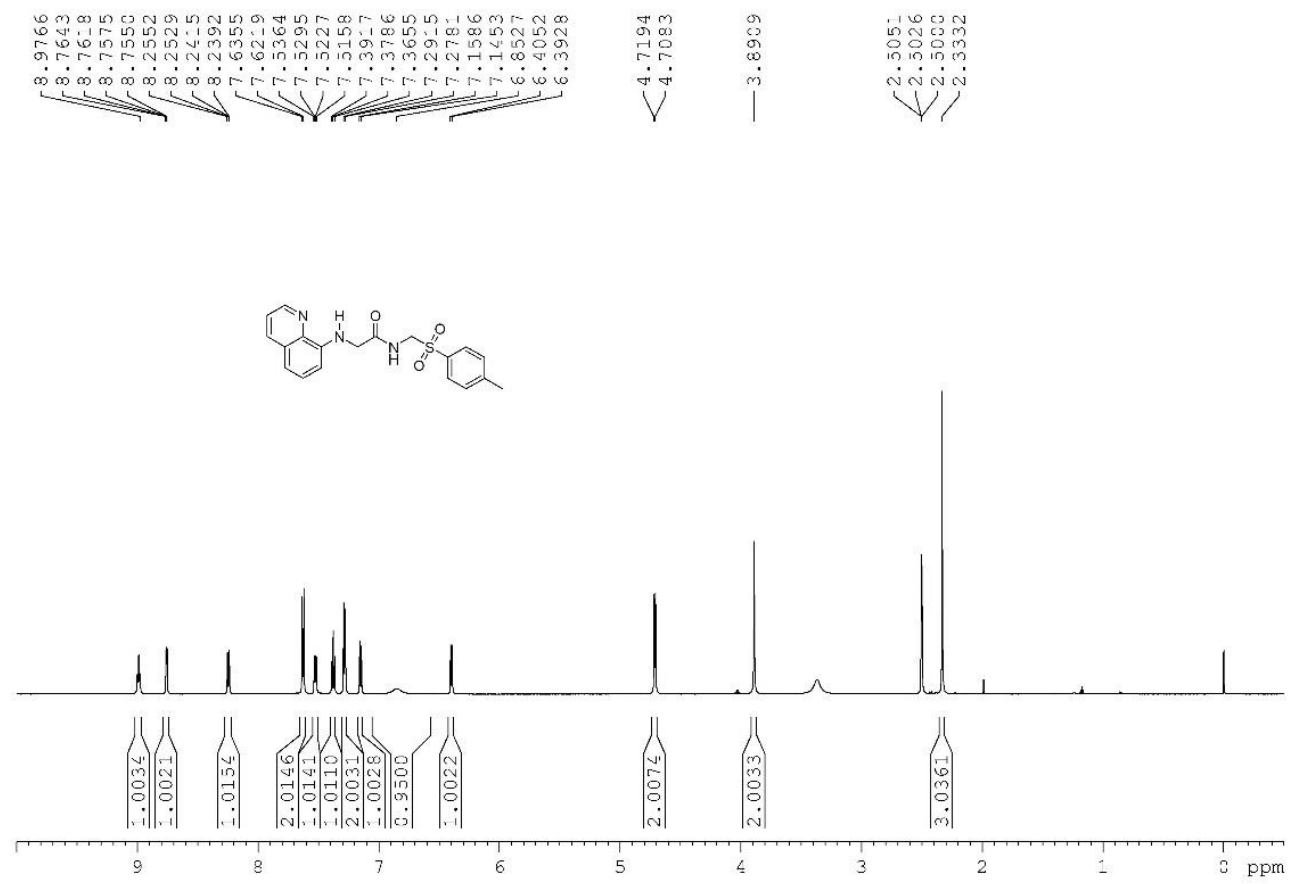

${ }^{13} \mathrm{C}\left\{{ }^{1} \mathrm{H}\right\}$ NMR (151 MHz, $d_{6}$-DMSO) of compound $\mathbf{3 z}$
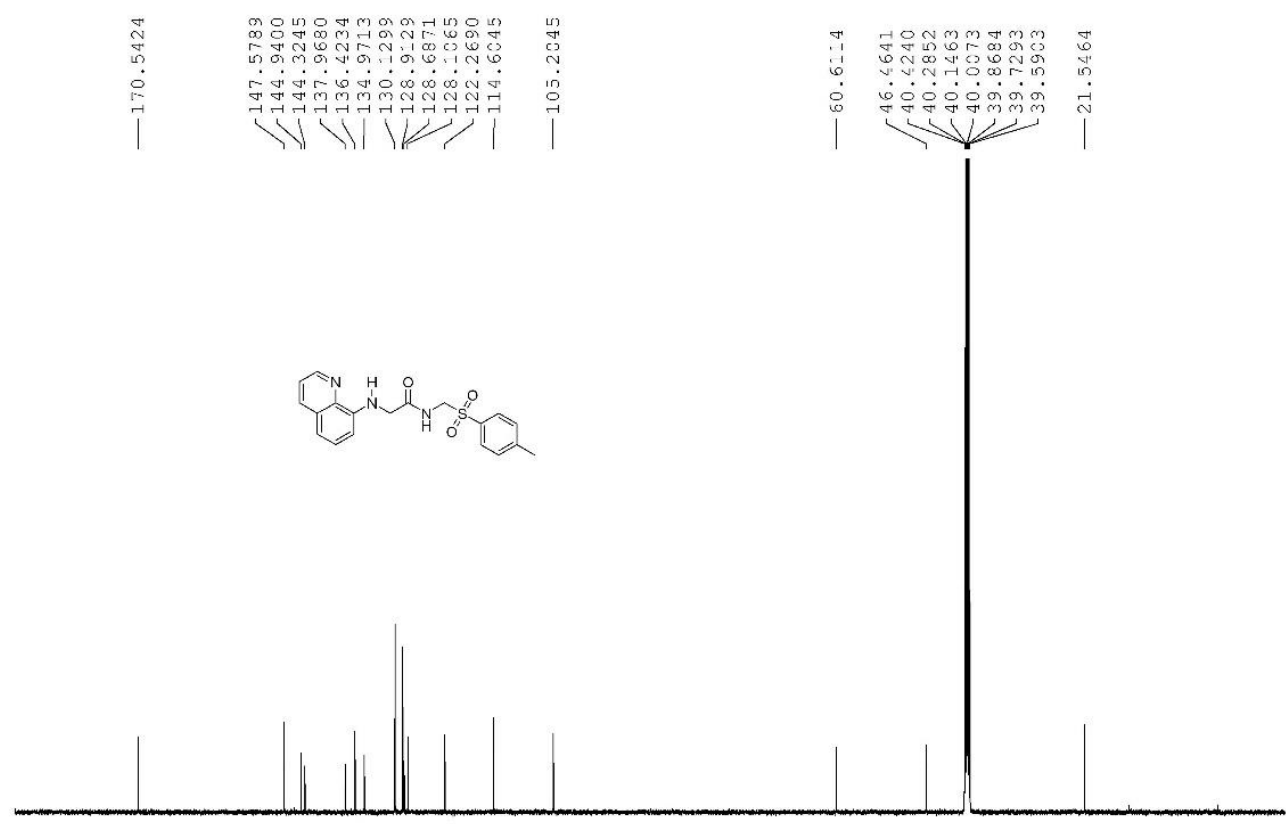

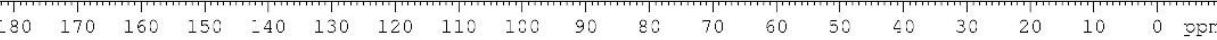


${ }^{1} \mathrm{H}$ NMR $\left(600 \mathrm{MHz}, \mathrm{CDCl}_{3}\right)$ of compound 3aa
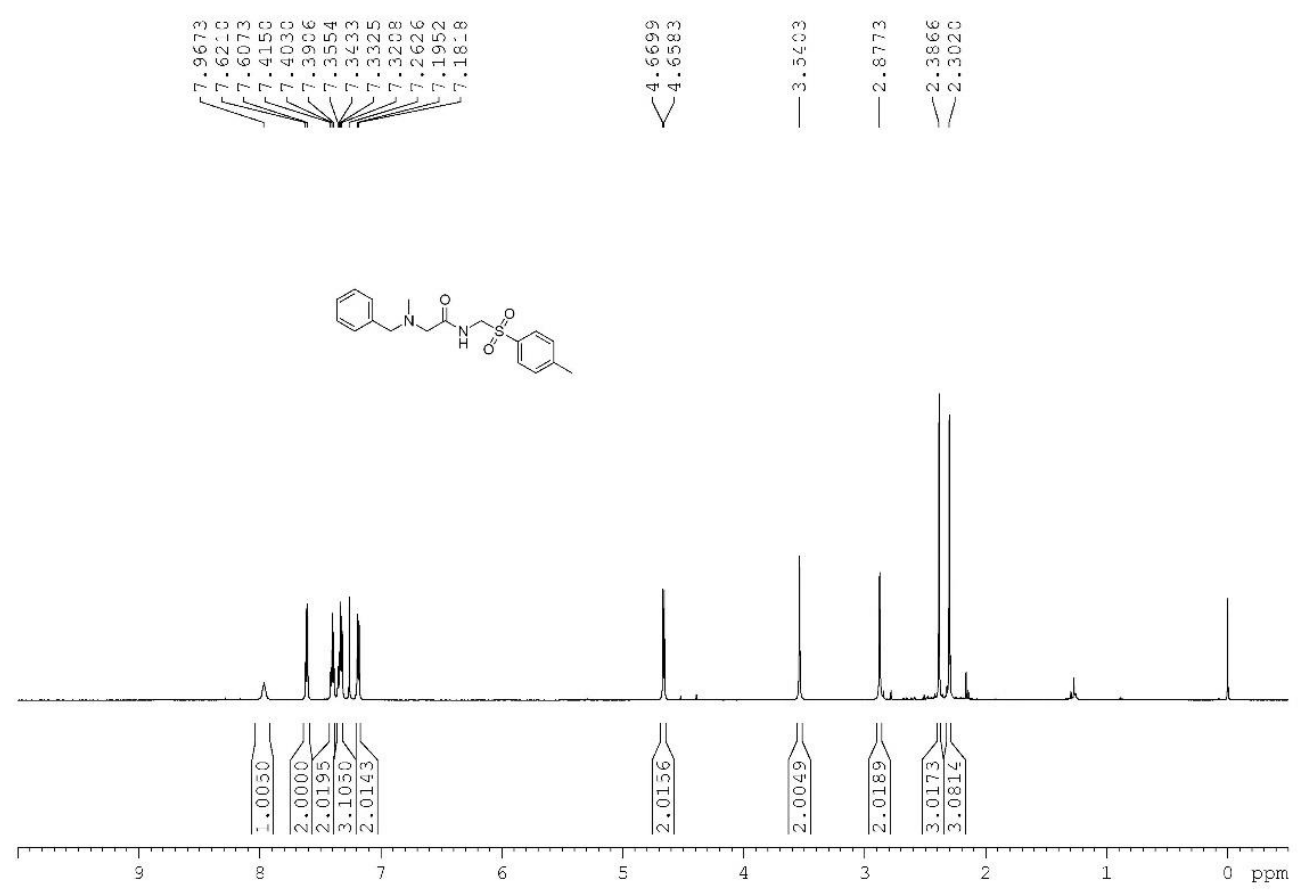

${ }^{13} \mathrm{C}\left\{{ }^{1} \mathrm{H}\right\}$ NMR (151 MHz, $\left.\mathrm{CDCl}_{3}\right)$ of compound 3aa

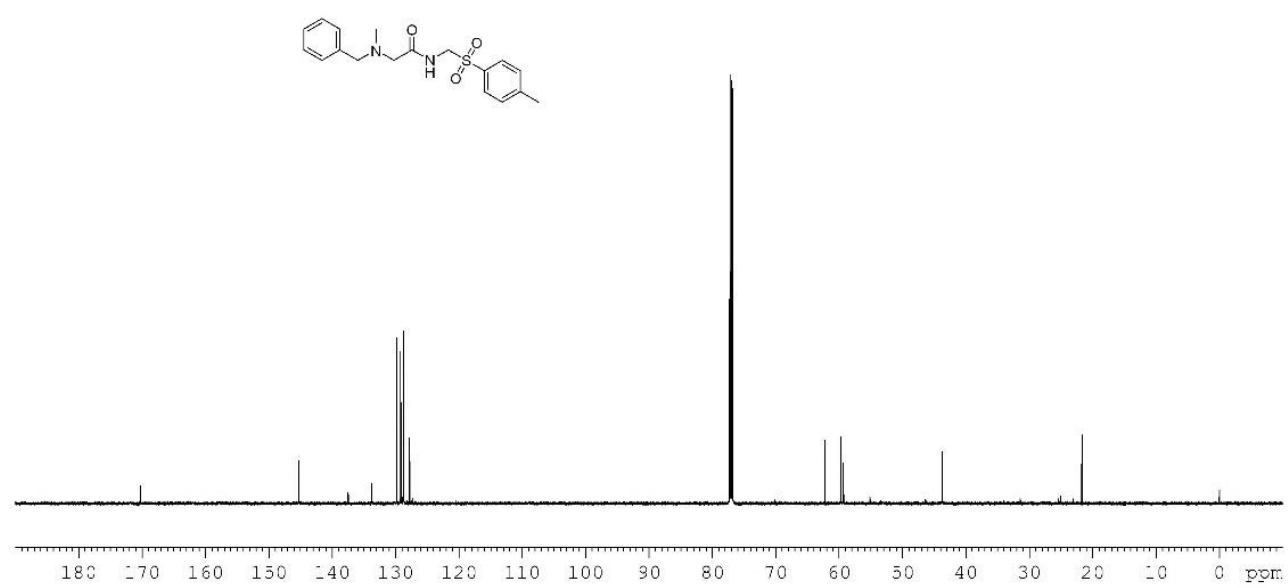


${ }^{1} \mathrm{H}$ NMR $\left(400 \mathrm{MHz}, \mathrm{CDCl}_{3}\right)$ of compound $\mathbf{5 a}$
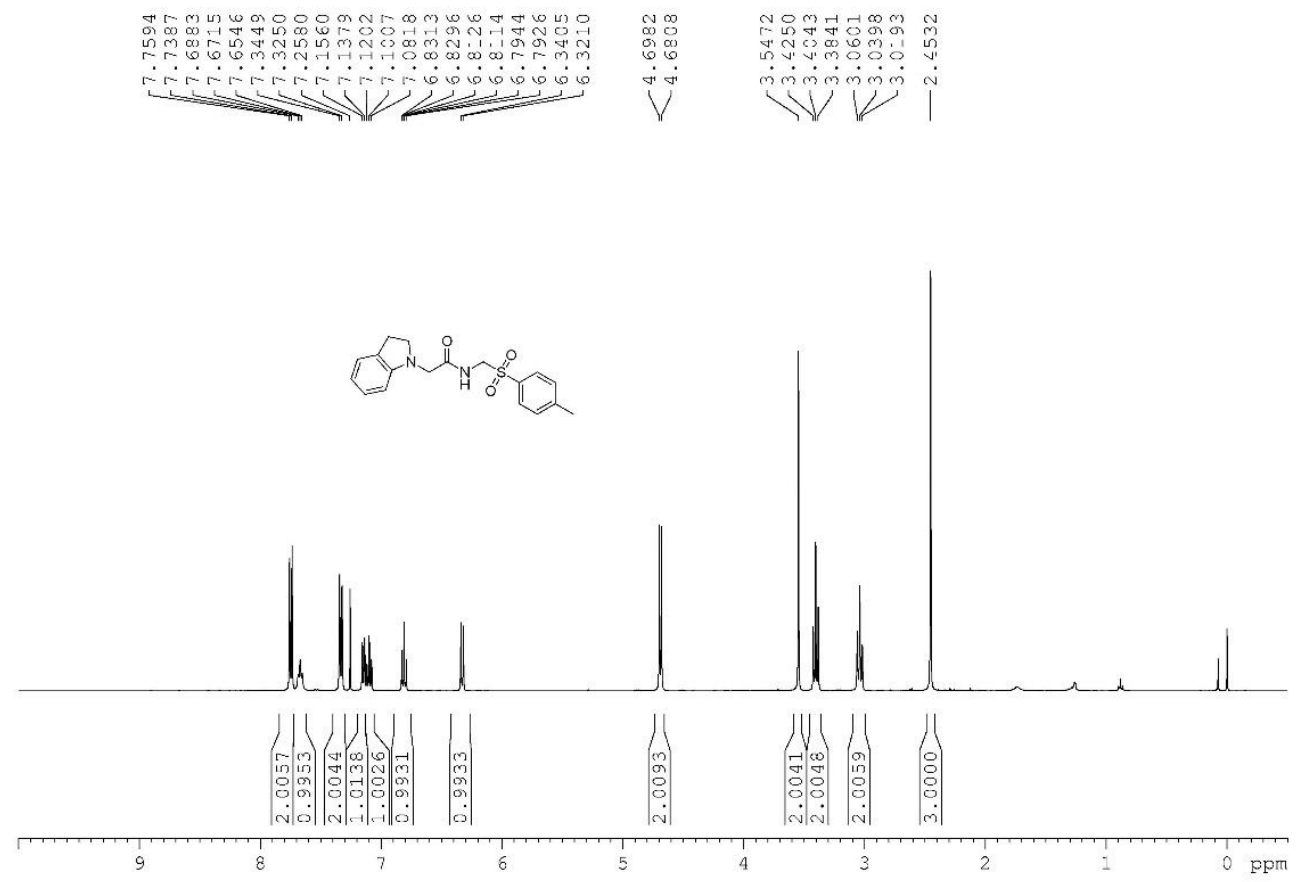

${ }^{13} \mathrm{C}\left\{{ }^{1} \mathrm{H}\right\}$ NMR (101 MHz, $\left.\mathrm{CDCl}_{3}\right)$ of compound $\mathbf{5 a}$
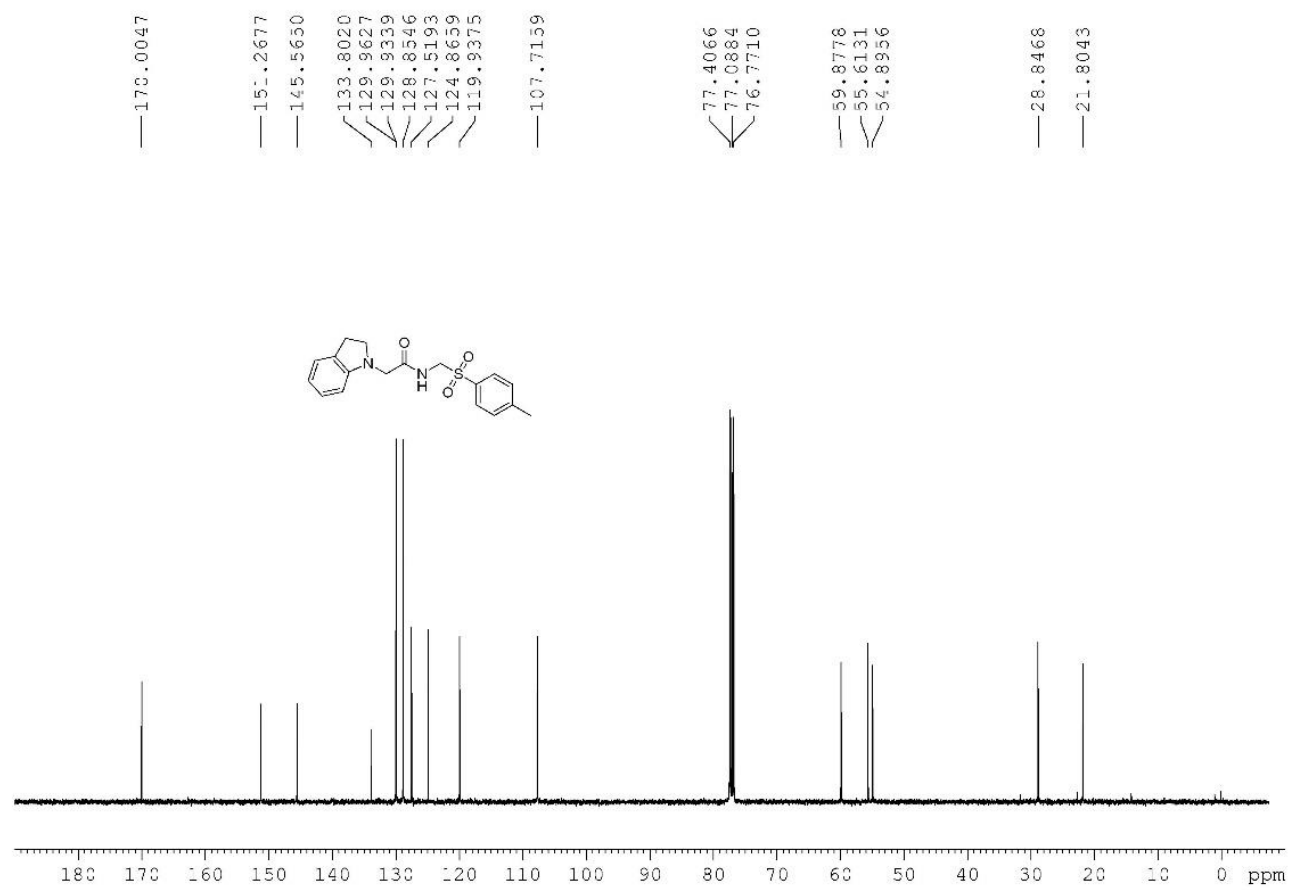
${ }^{1} \mathrm{H}$ NMR $\left(600 \mathrm{MHz}, \mathrm{CDCl}_{3}\right)$ of compound $\mathbf{5 b}$

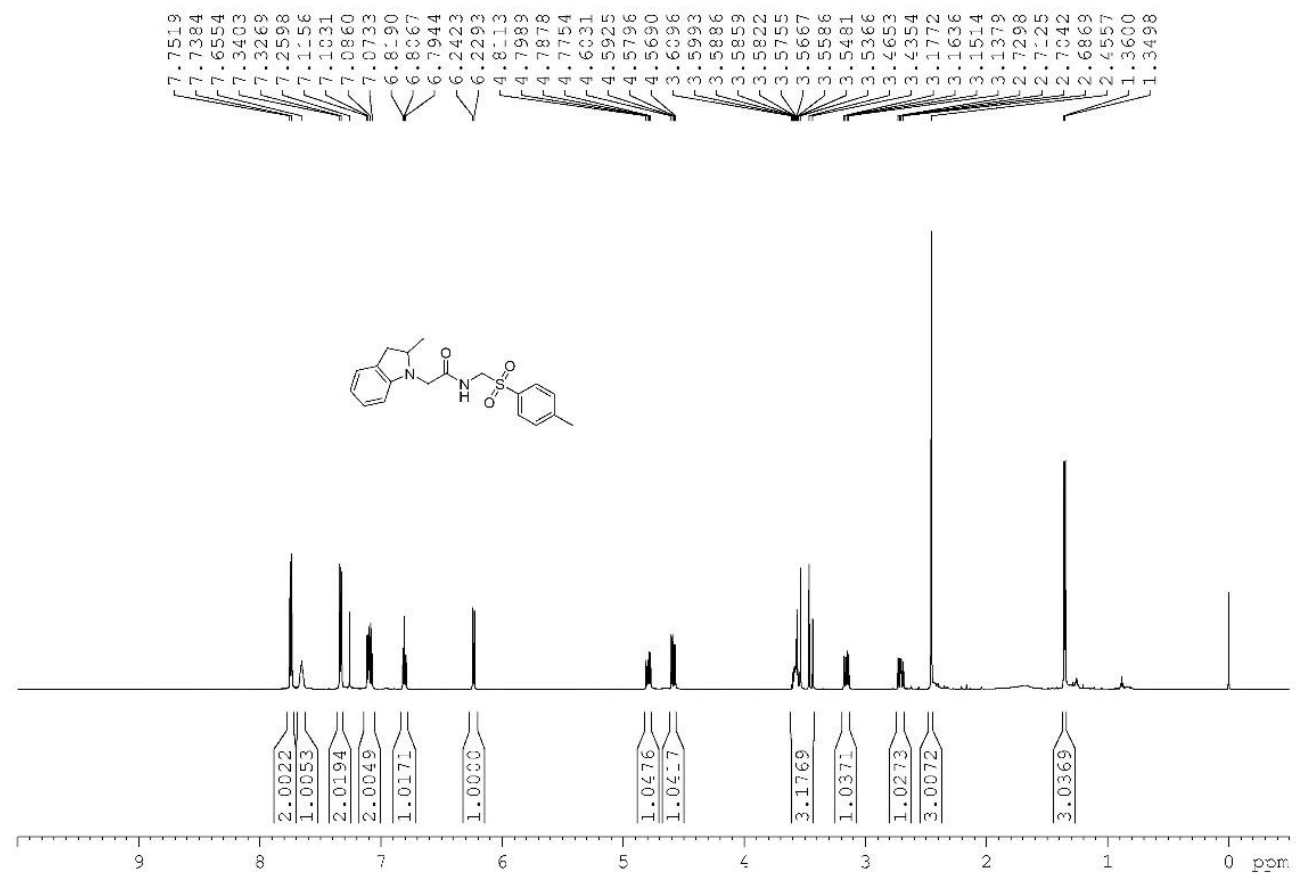

${ }^{13} \mathrm{C}\left\{{ }^{1} \mathrm{H}\right\}$ NMR (151 MHz, $\left.\mathrm{CDCl}_{3}\right)$ of compound $\mathbf{5 b}$
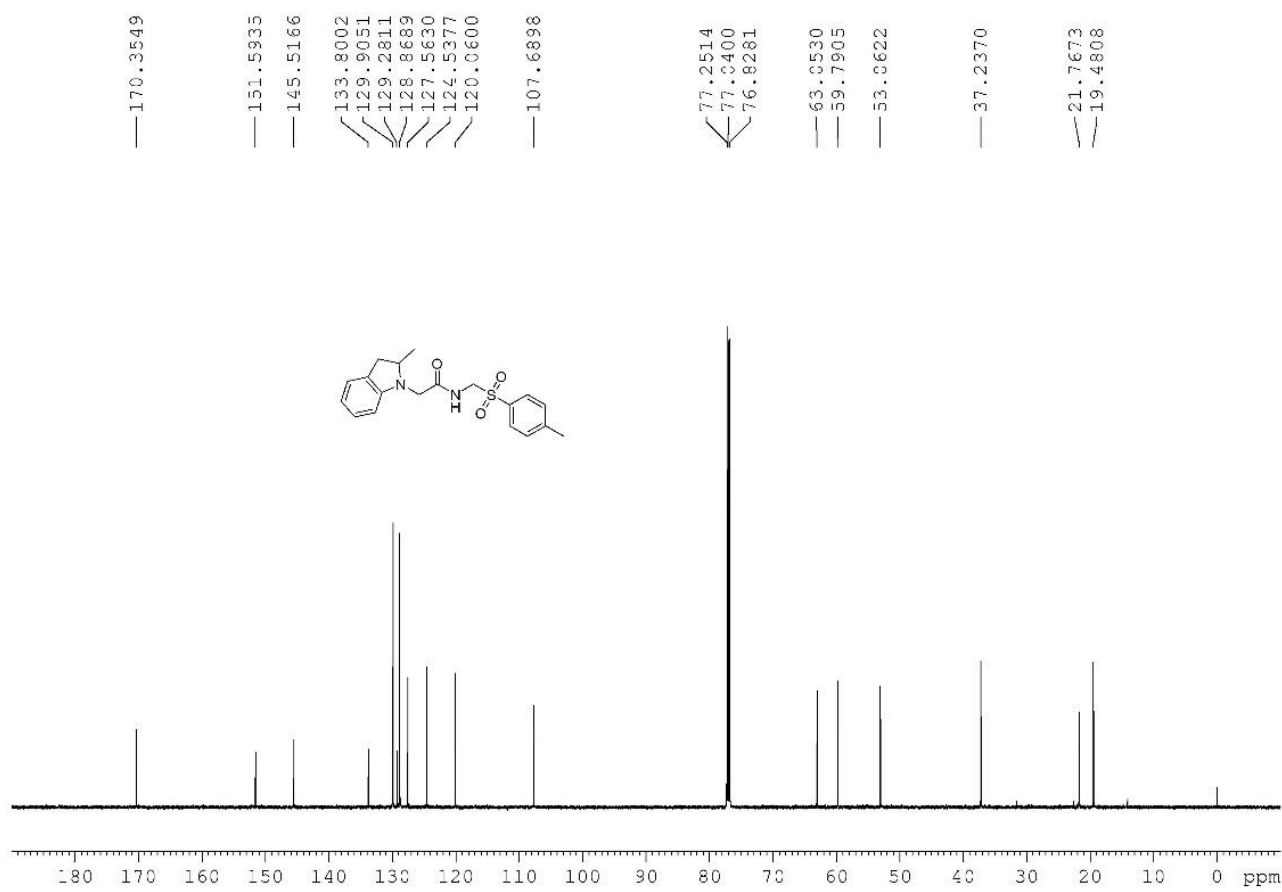
${ }^{1} \mathrm{H}$ NMR $\left(600 \mathrm{MHz}, \mathrm{CDCl}_{3}\right)$ of compound $\mathbf{5 c}$
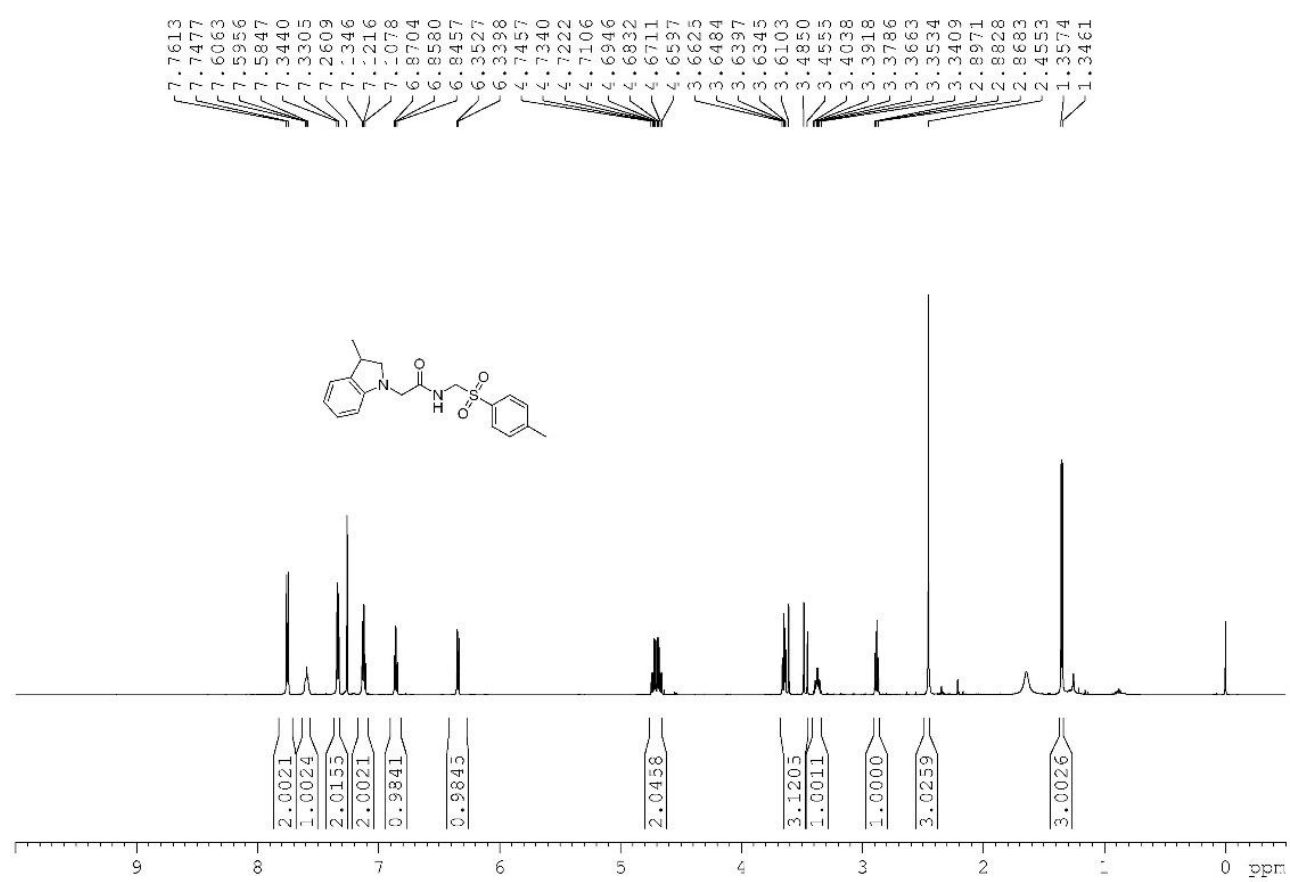

${ }^{13} \mathrm{C}\left\{{ }^{1} \mathrm{H}\right\}$ NMR $\left(151 \mathrm{MHz}, \mathrm{CDCl}_{3}\right)$ of compound $\mathbf{5 c}$

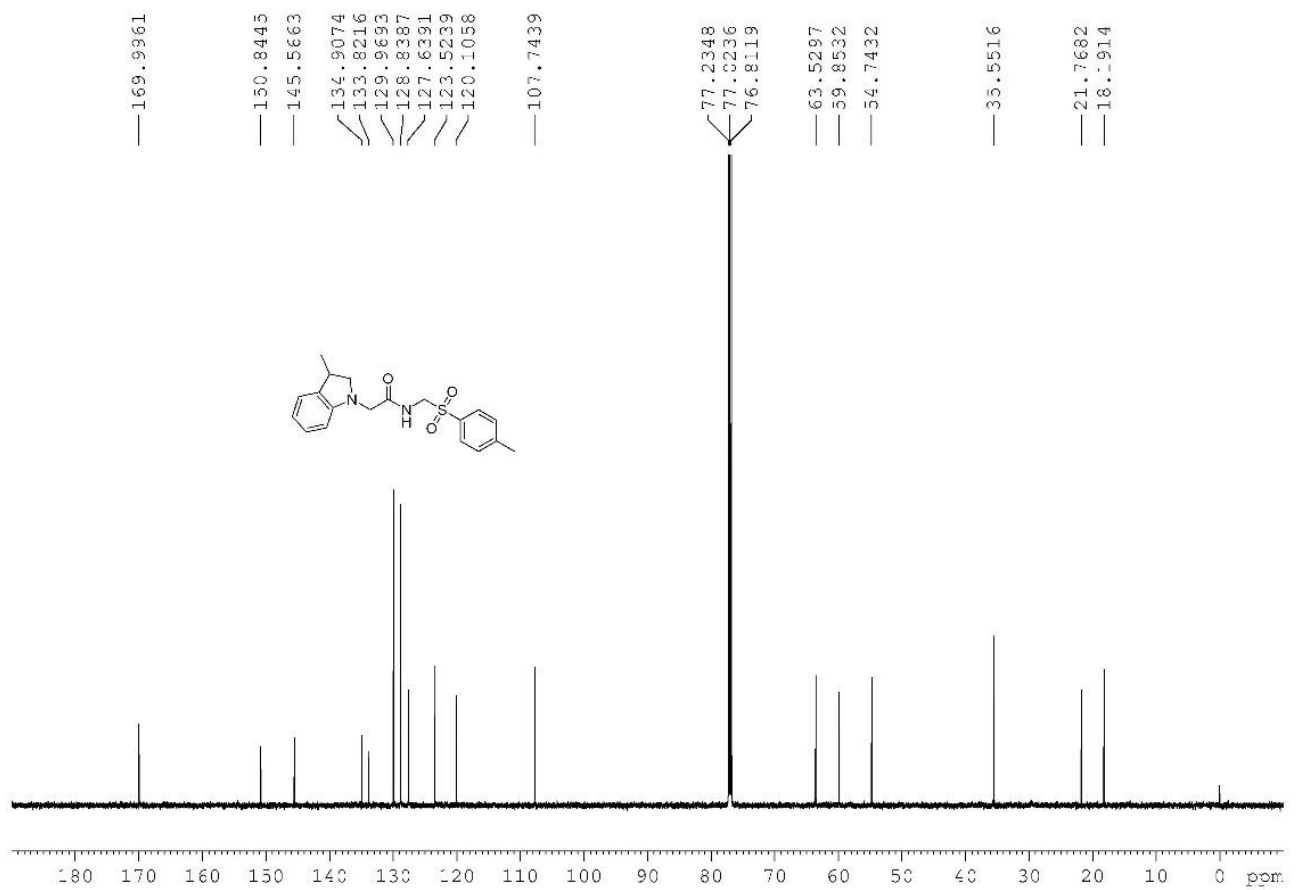


${ }^{1} \mathrm{H}$ NMR (600 MHz, $\left.\mathrm{CDCl}_{3}\right)$ of compound $\mathbf{5 d}$
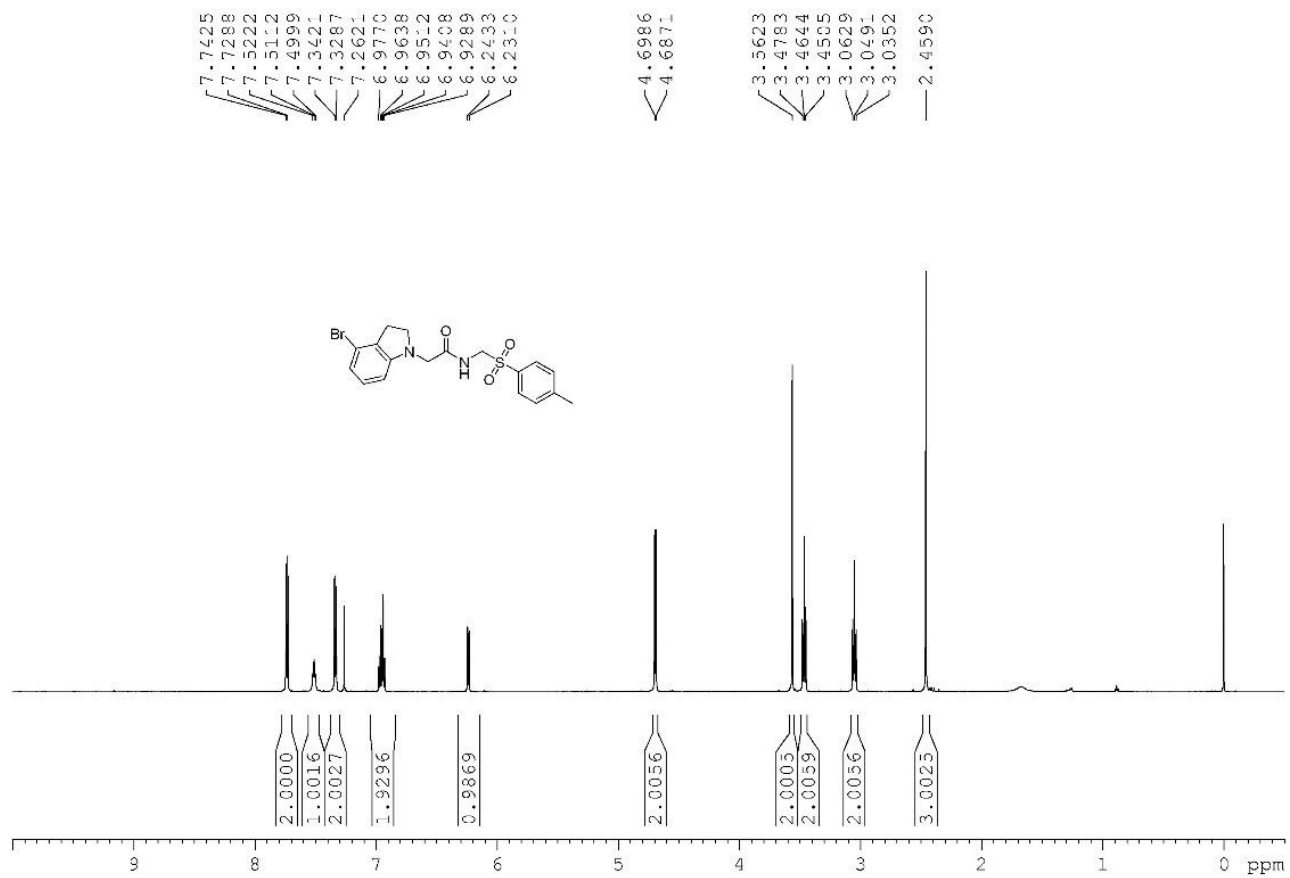

${ }^{13} \mathrm{C}\left\{{ }^{1} \mathrm{H}\right\}$ NMR $\left(151 \mathrm{MHz}, \mathrm{CDCl}_{3}\right)$ of compound $\mathbf{5 d}$

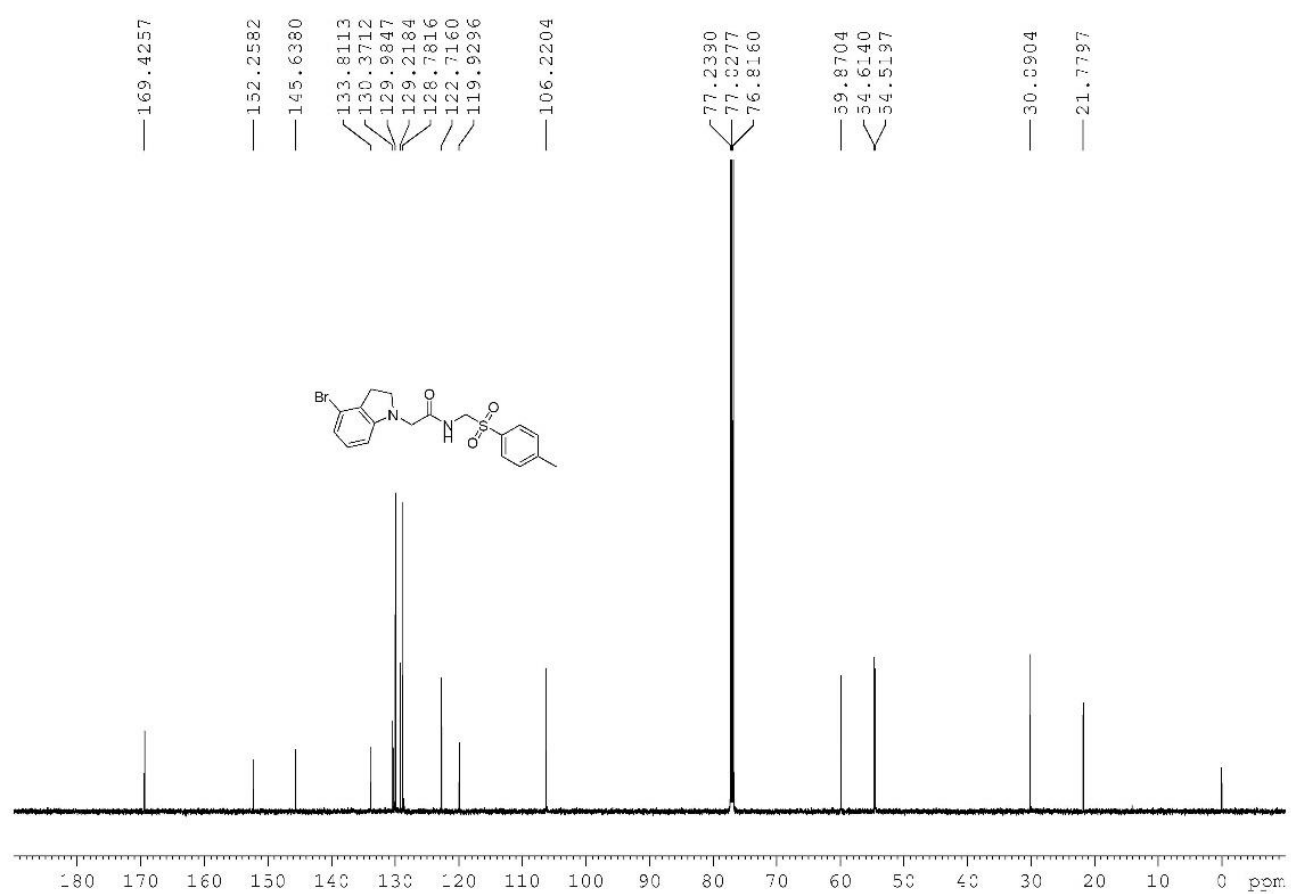


${ }^{1} \mathrm{H}$ NMR $\left(600 \mathrm{MHz}, \mathrm{CDCl}_{3}\right)$ of compound $\mathbf{5 f}$

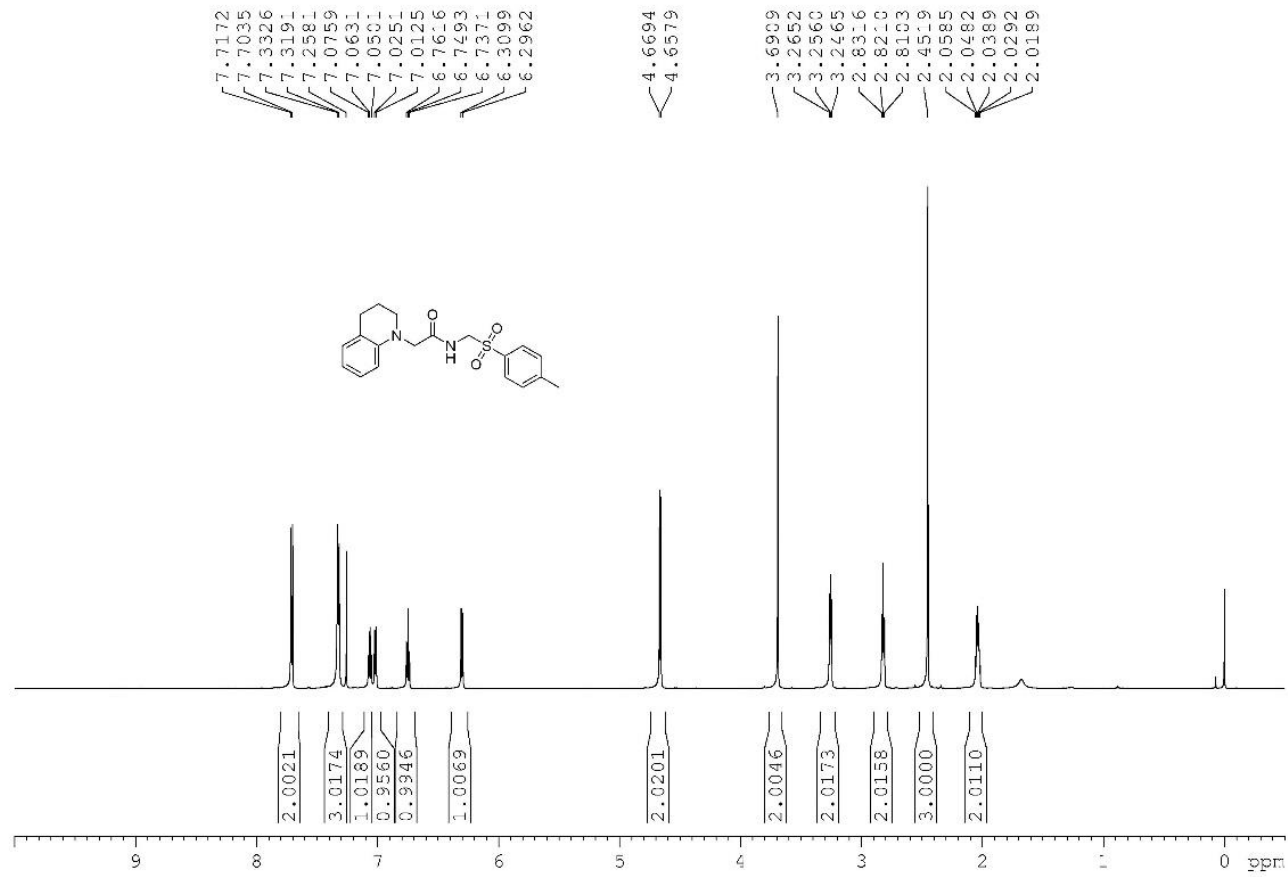

${ }^{13} \mathrm{C}\left\{{ }^{1} \mathrm{H}\right\}$ NMR (101 MHz, $\left.\mathrm{CDCl}_{3}\right)$ of compound $\mathbf{5 f}$

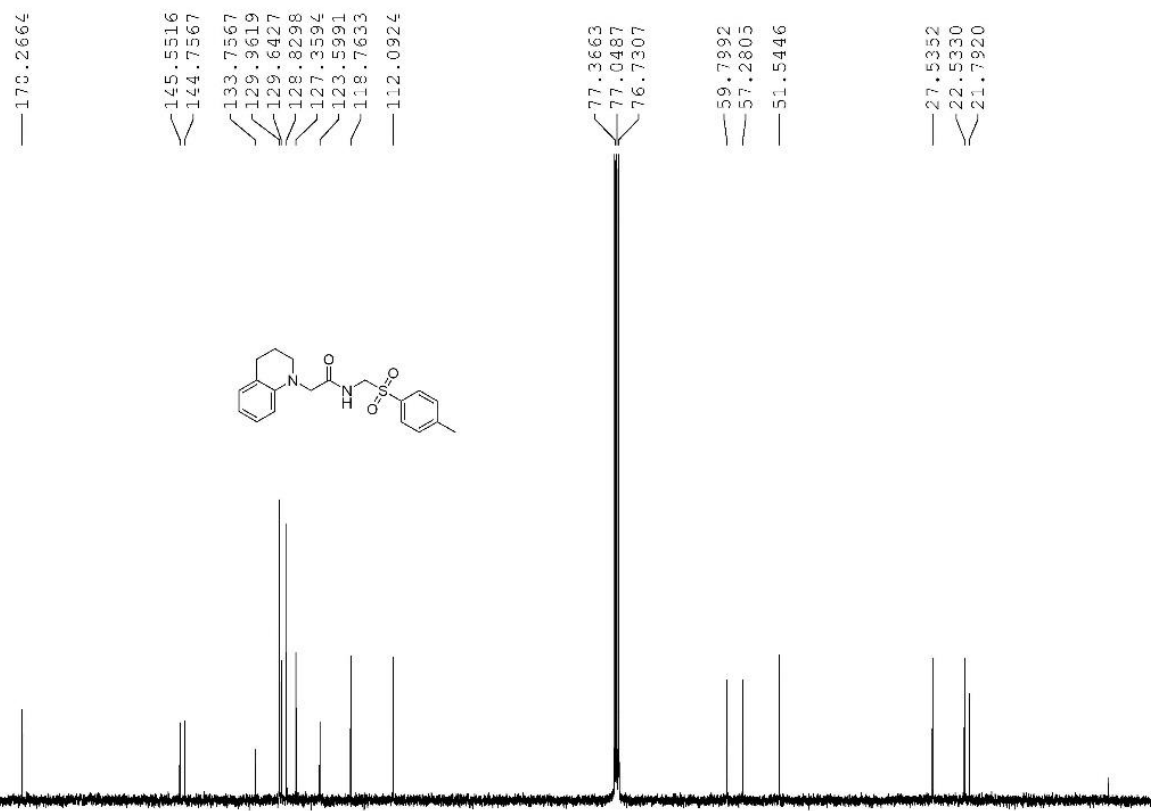

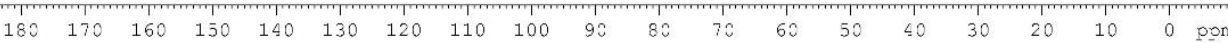


${ }^{1} \mathrm{H}$ NMR $\left(600 \mathrm{MHz}, \mathrm{CDCl}_{3}\right)$ of compound $\mathbf{5 g}$
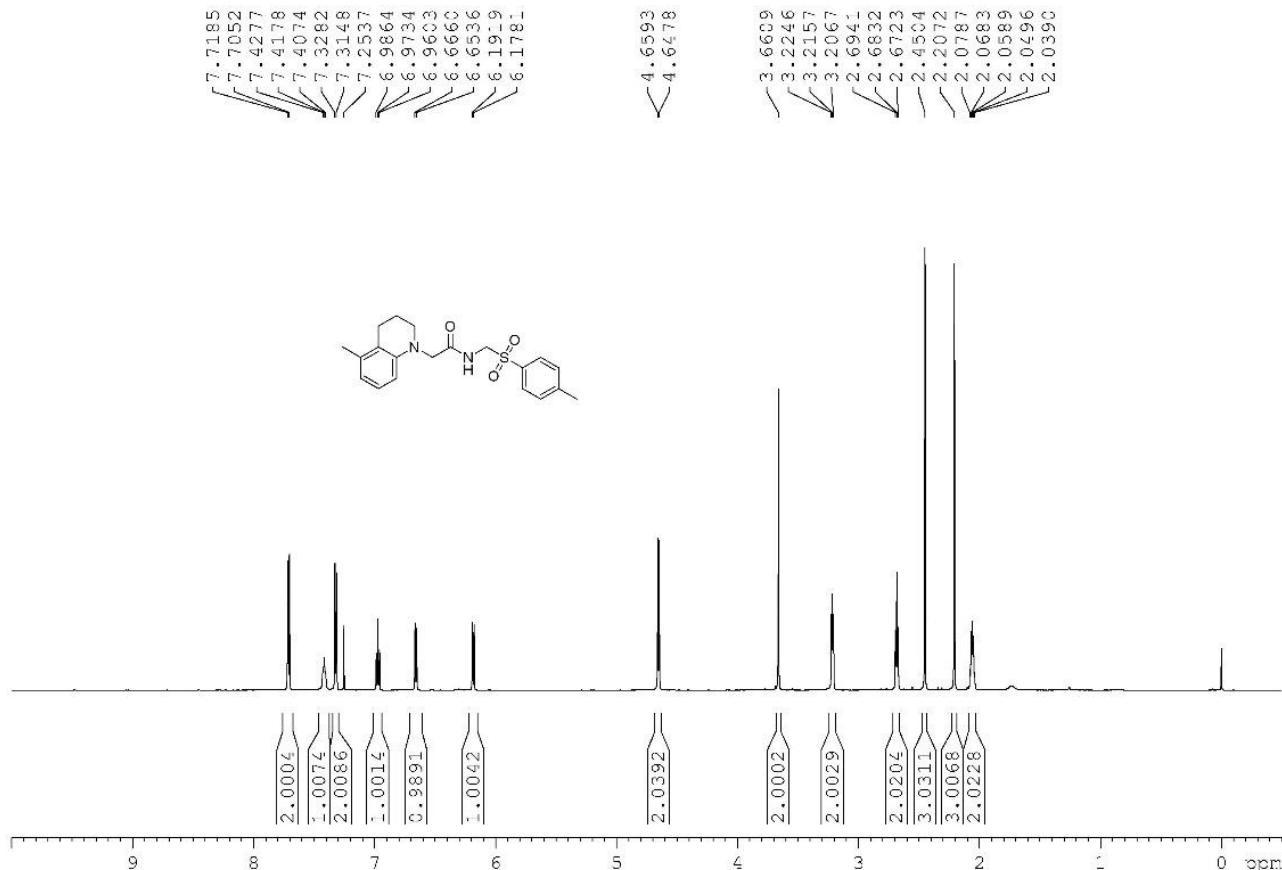

${ }^{13} \mathrm{C}\left\{{ }^{1} \mathrm{H}\right\}$ NMR (151 MHz, $\left.\mathrm{CDCl}_{3}\right)$ of compound $\mathbf{5 g}$
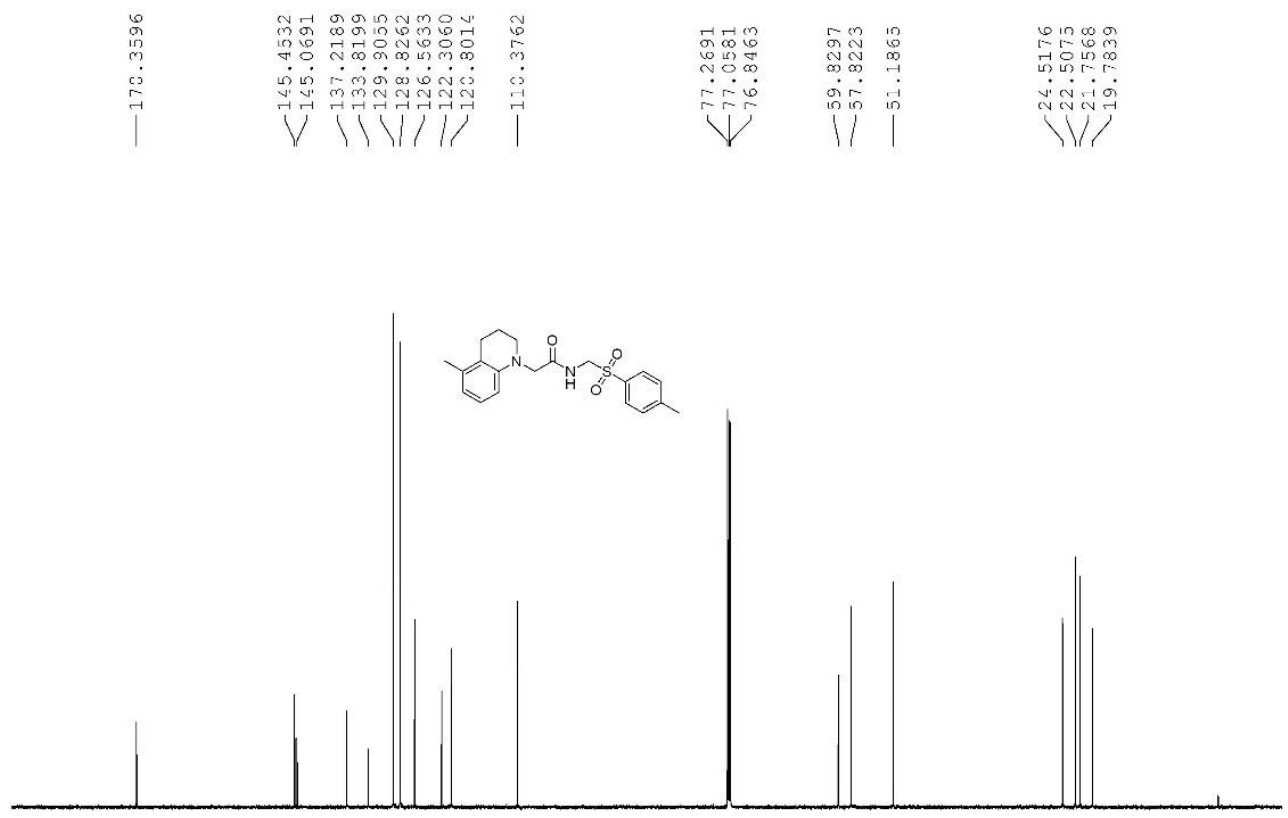

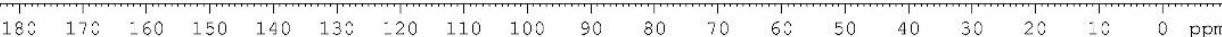


${ }^{1} \mathrm{H}$ NMR (600 MHz, $\mathrm{CDCl}_{3}$ ) of compound $\mathbf{5 h}$

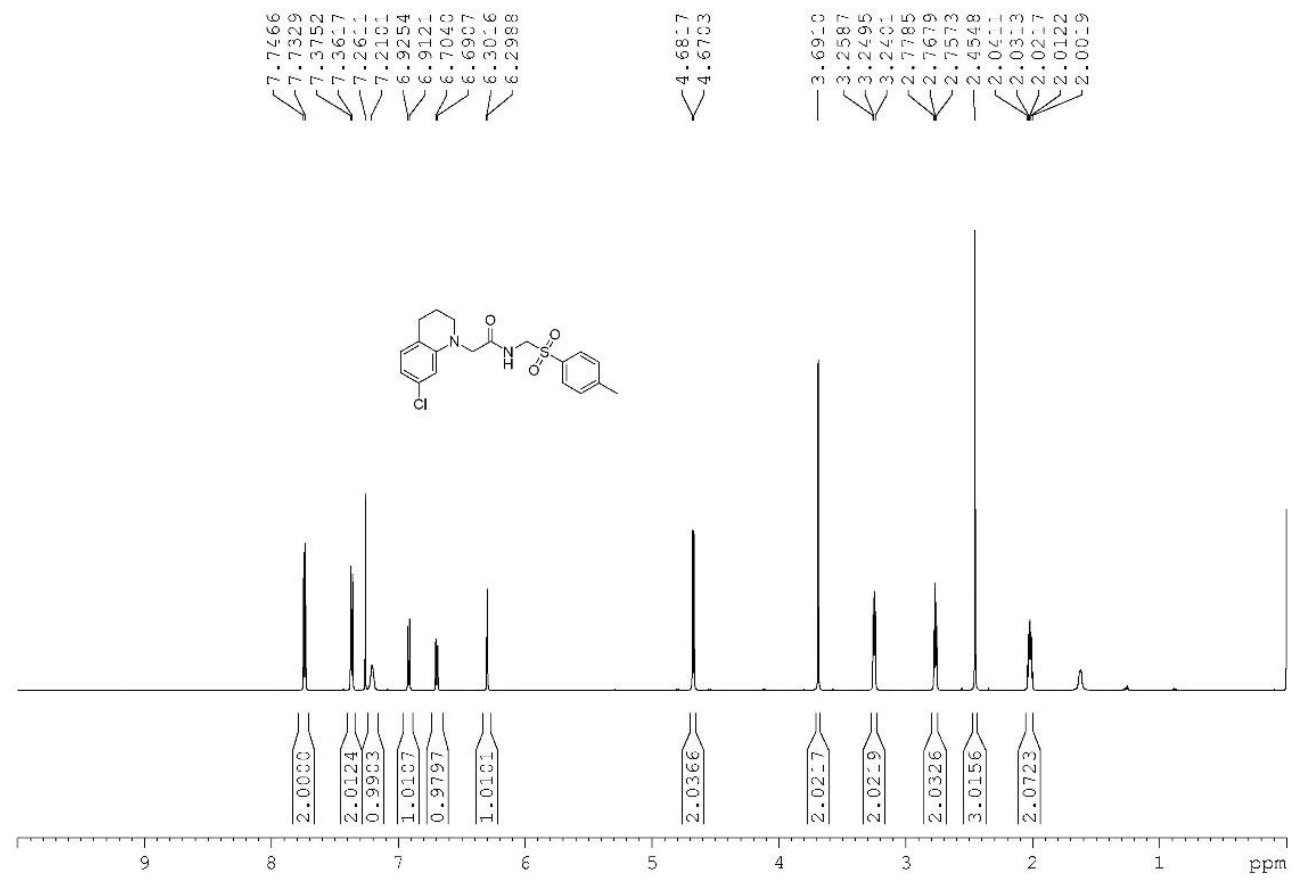

${ }^{13} \mathrm{C}\left\{{ }^{1} \mathrm{H}\right\}$ NMR (151 MHz, $\left.\mathrm{CDCl}_{3}\right)$ of compound $\mathbf{5 h}$

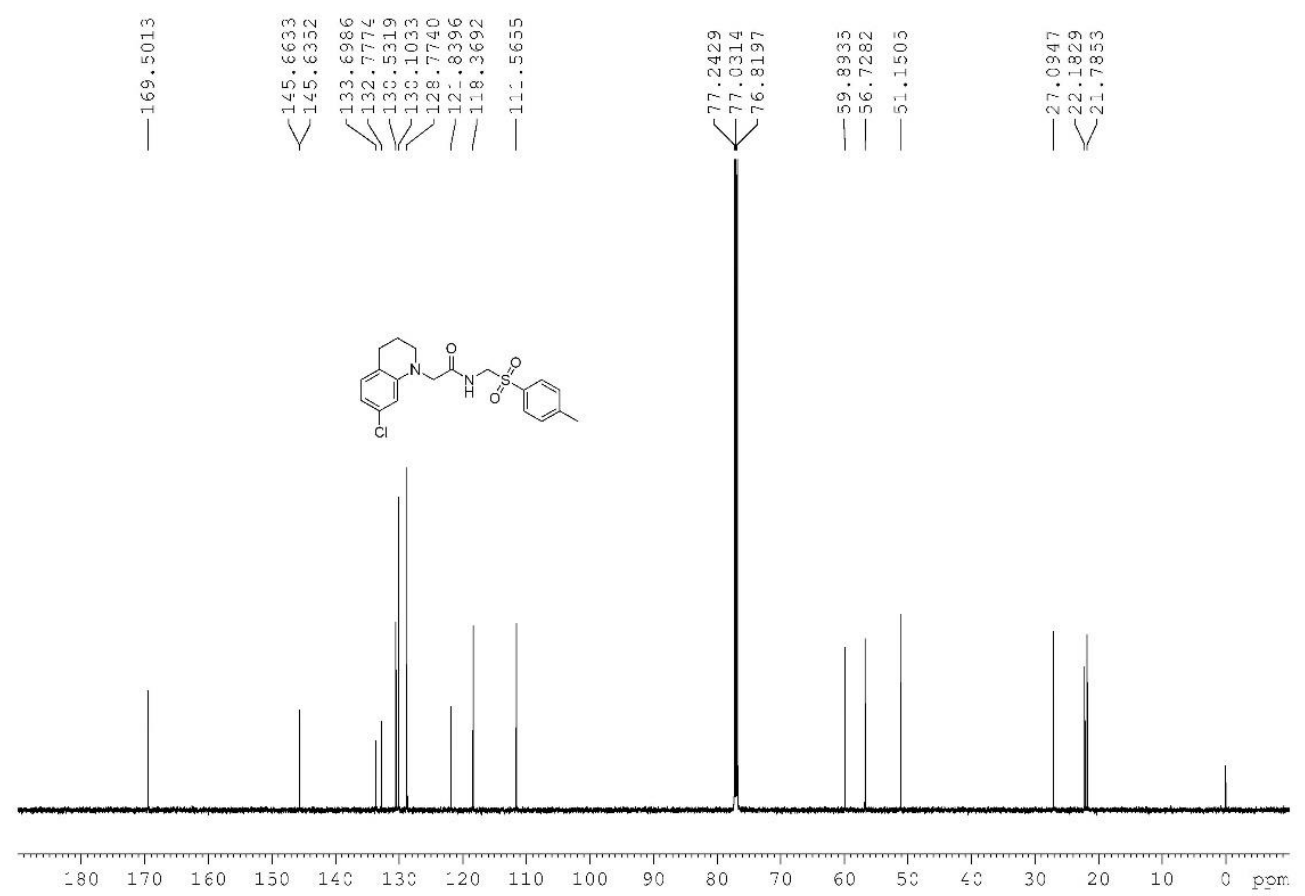


${ }^{1} \mathrm{H}$ NMR $\left(600 \mathrm{MHz}, \mathrm{CDCl}_{3}\right)$ of compound $\mathbf{5 i}$

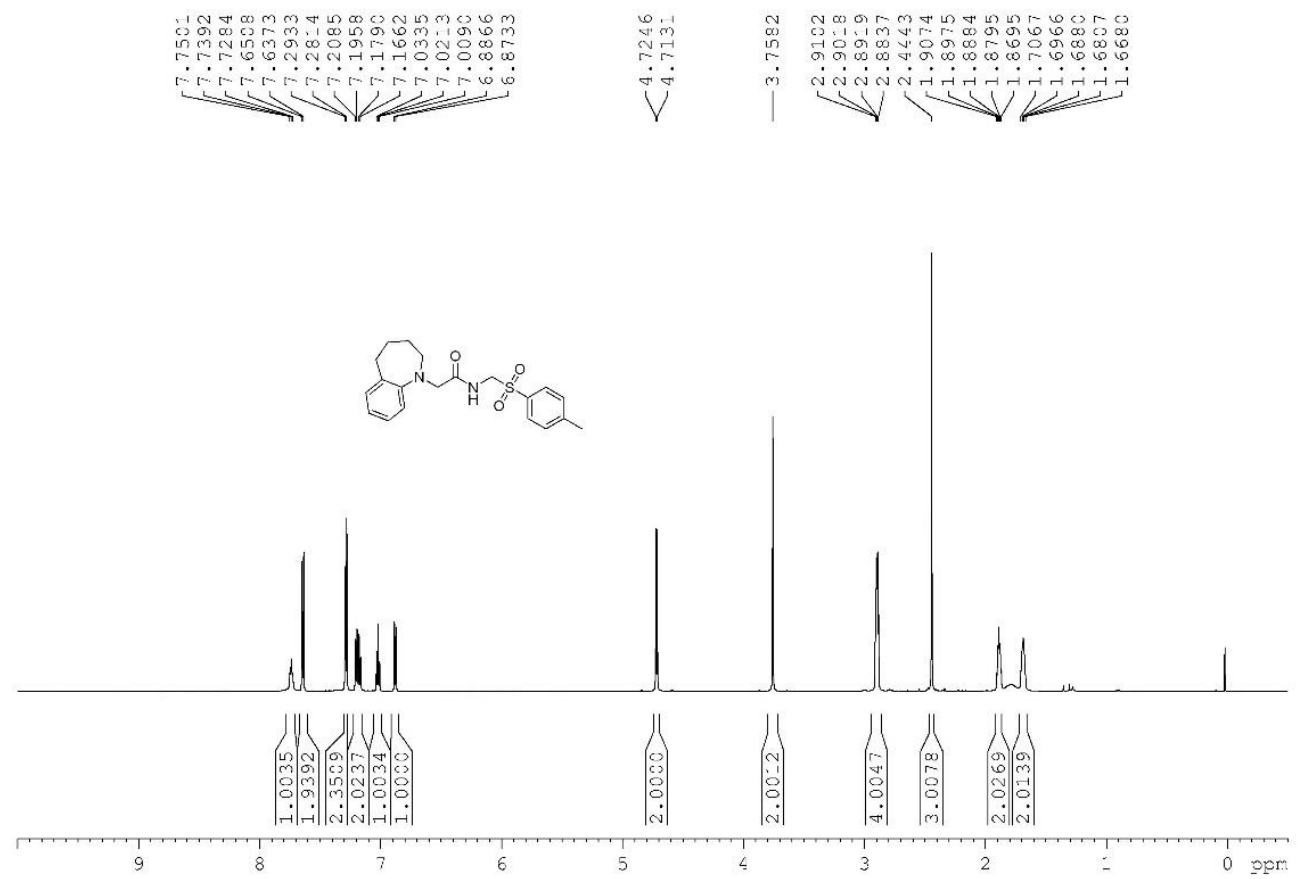

${ }^{13} \mathrm{C}\left\{{ }^{1} \mathrm{H}\right\}$ NMR $\left(151 \mathrm{MHz}, \mathrm{CDCl}_{3}\right)$ of compound $\mathbf{5 i}$
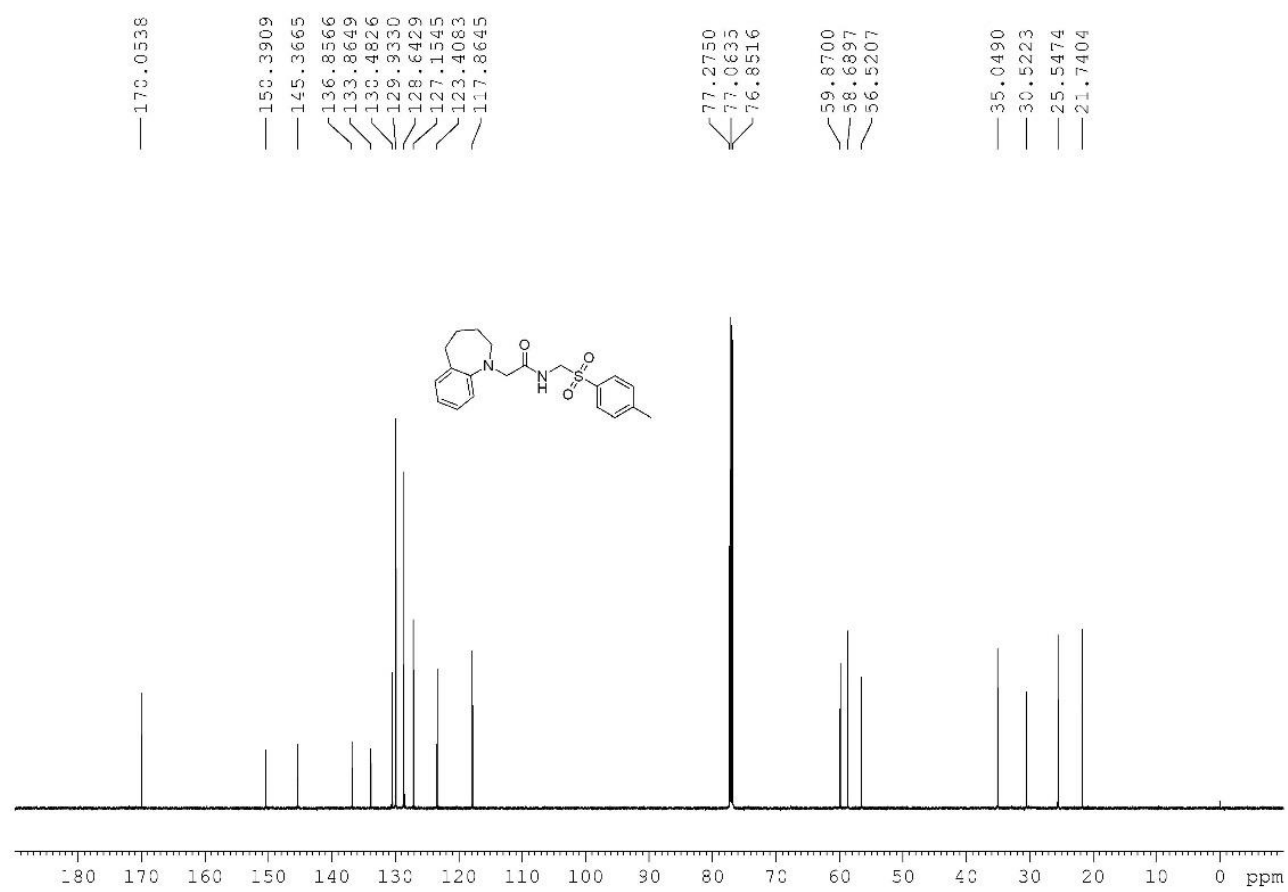
${ }^{1} \mathrm{H}$ NMR (600 MHz, $\left.\mathrm{CDCl}_{3}\right)$ of compound $\mathbf{5 j}$
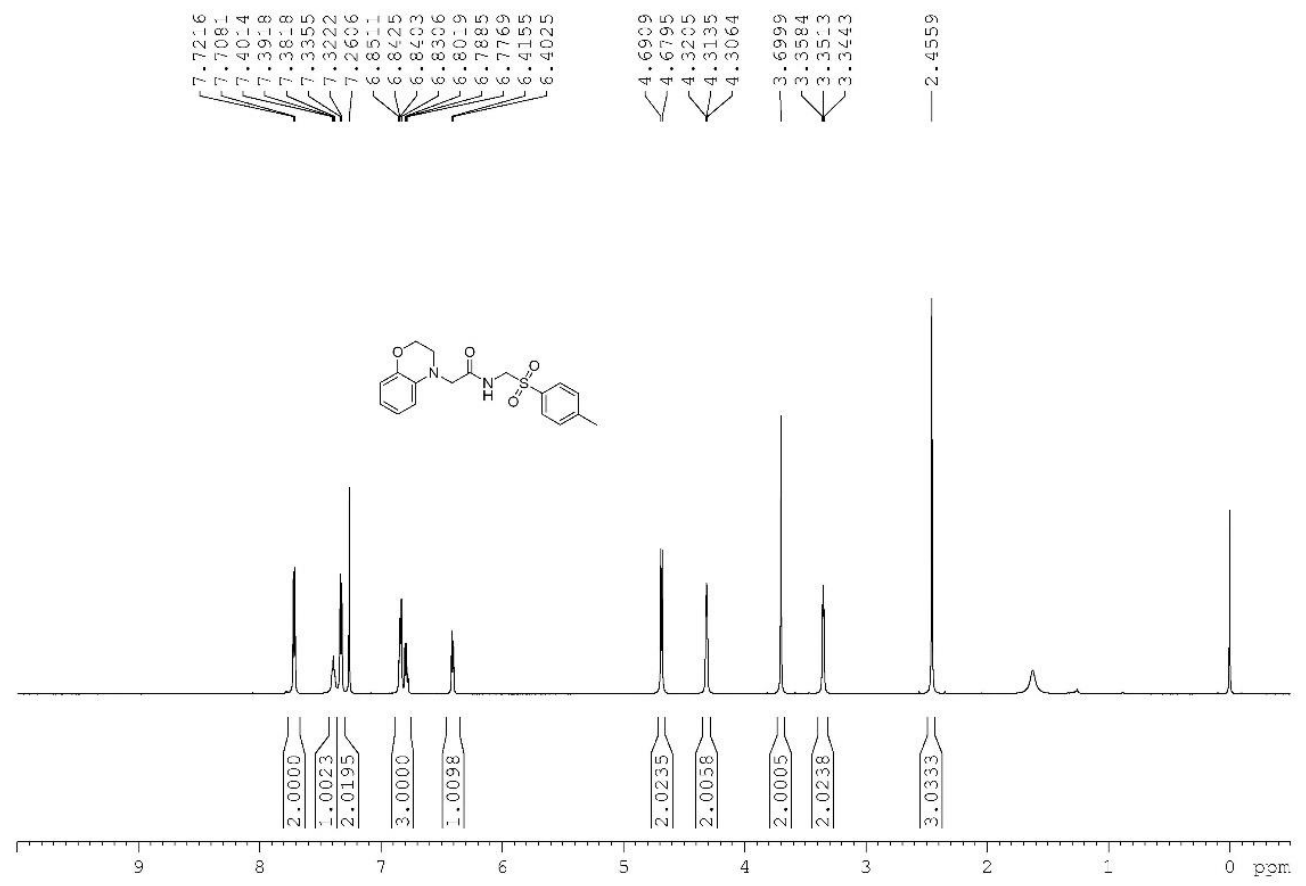

${ }^{13} \mathrm{C}\left\{{ }^{1} \mathrm{H}\right\}$ NMR (151 MHz, $\left.\mathrm{CDCl}_{3}\right)$ of compound $\mathbf{5 j}$

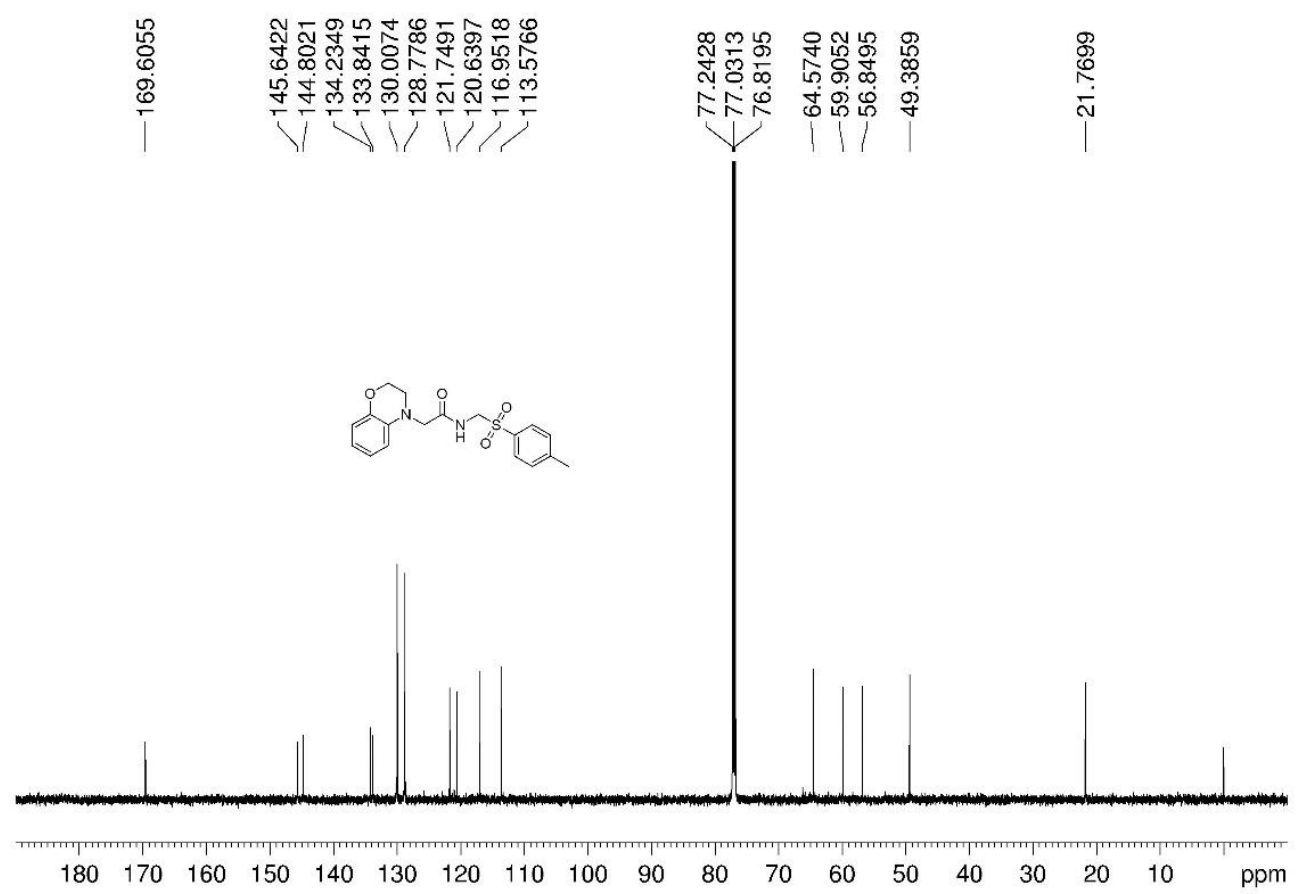


${ }^{1} \mathrm{H}$ NMR $\left(600 \mathrm{MHz}, \mathrm{CDCl}_{3}\right)$ of compound $\mathbf{5 k}$
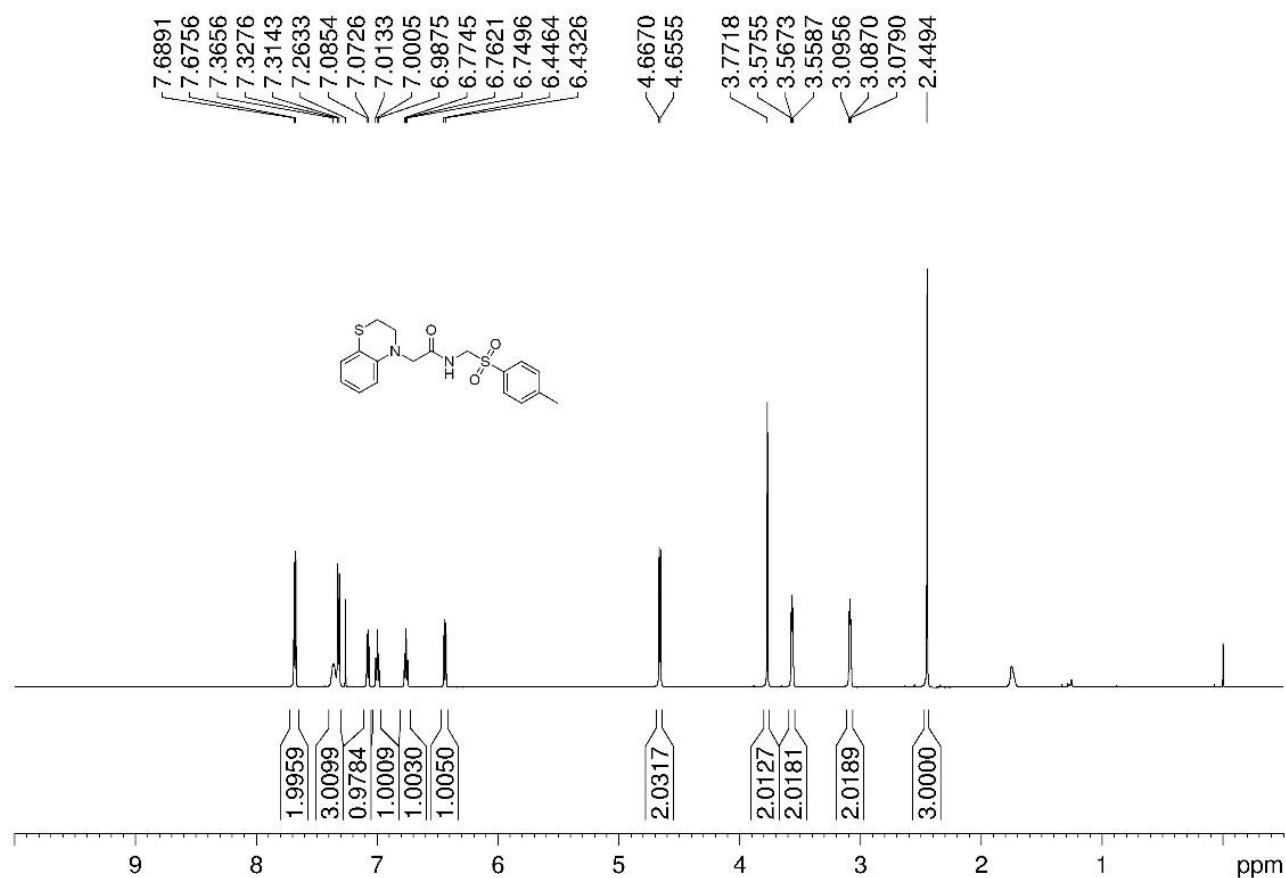

${ }^{13} \mathrm{C}\left\{{ }^{1} \mathrm{H}\right\}$ NMR (151 MHz, $\left.\mathrm{CDCl}_{3}\right)$ of compound $\mathbf{5 k}$
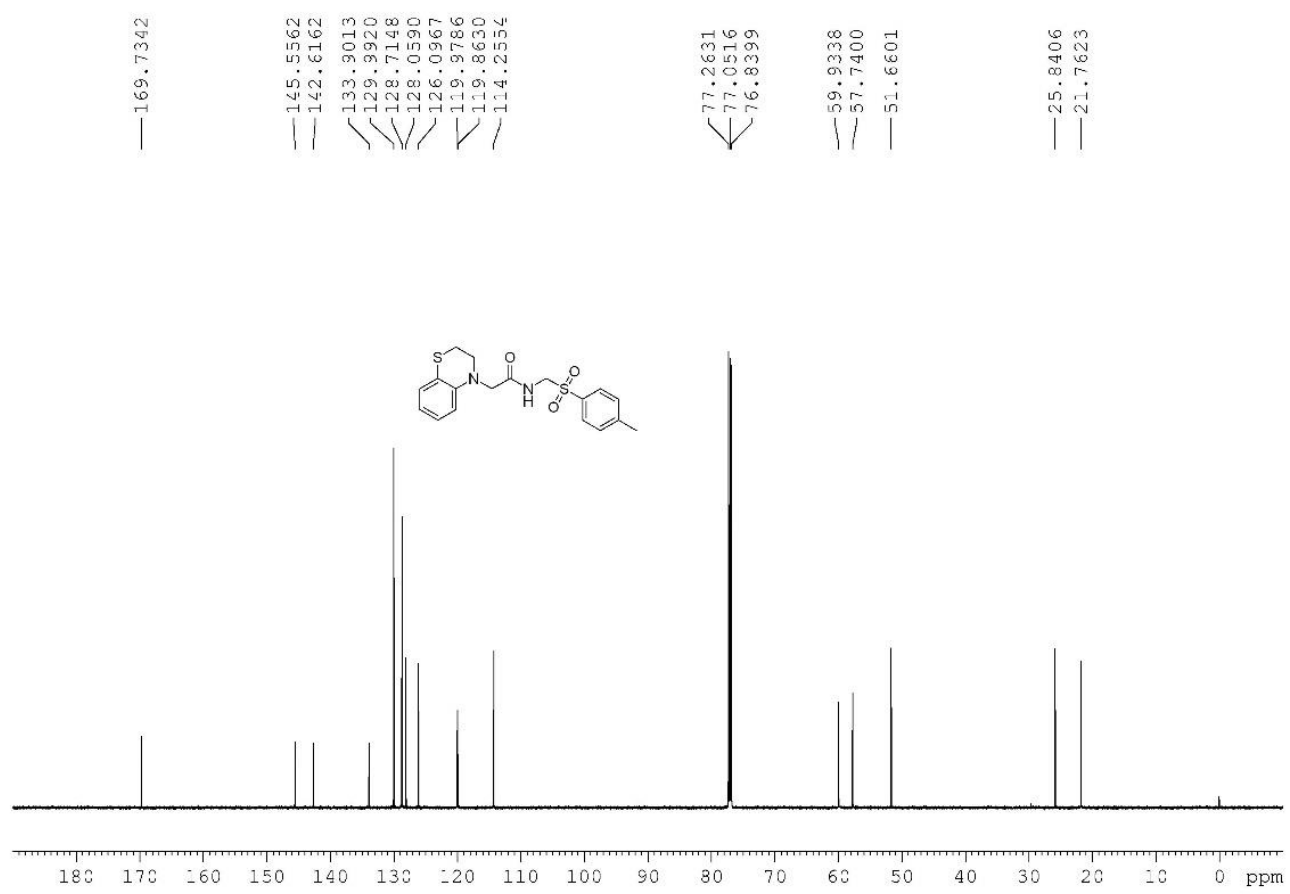
${ }^{1} \mathrm{H}$ NMR (600 MHz, $\mathrm{CDCl}_{3}$ ) of compound $\mathbf{5 l}$

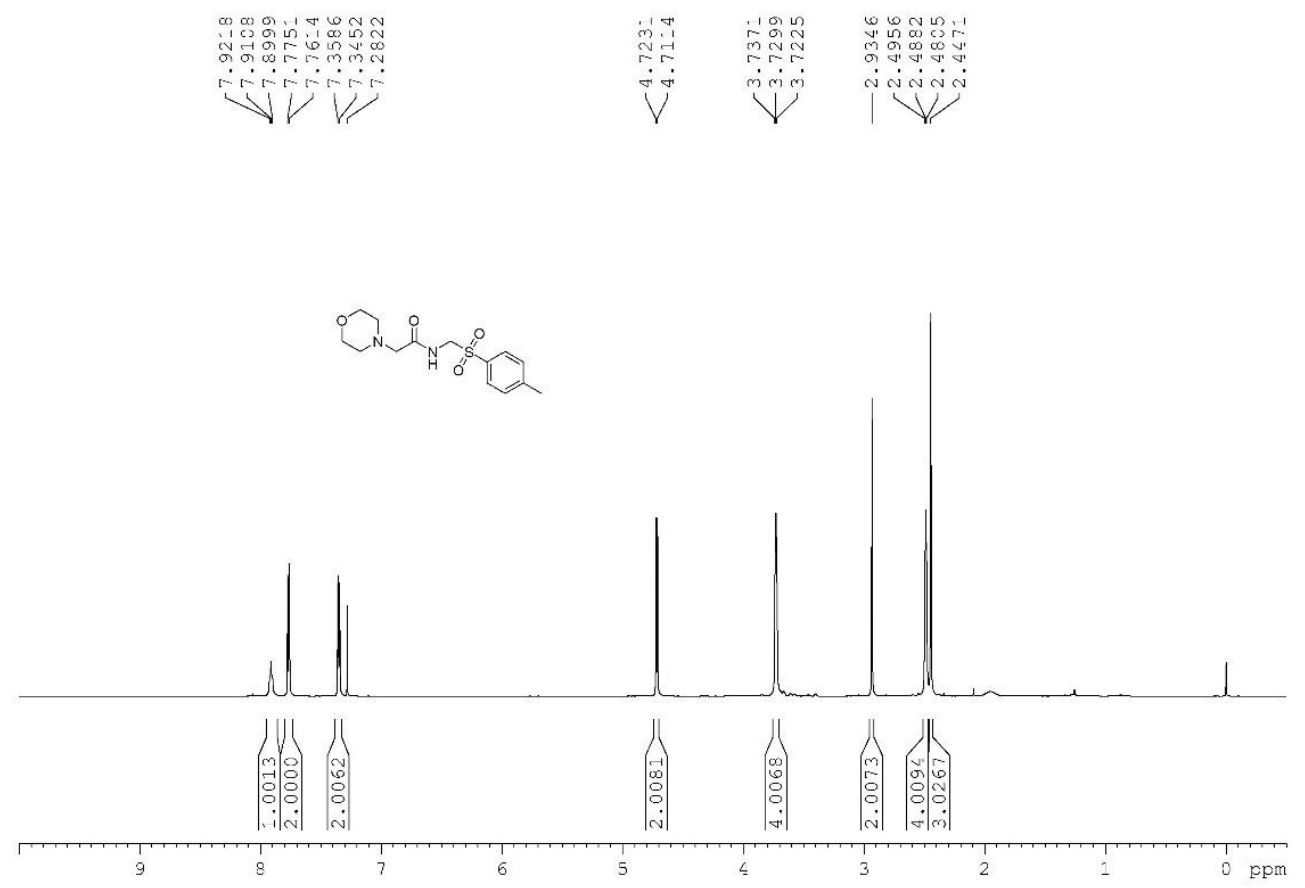

${ }^{13} \mathrm{C}\left\{{ }^{1} \mathrm{H}\right\}$ NMR $\left(151 \mathrm{MHz}, \mathrm{CDCl}_{3}\right)$ of compound $\mathbf{5 l}$
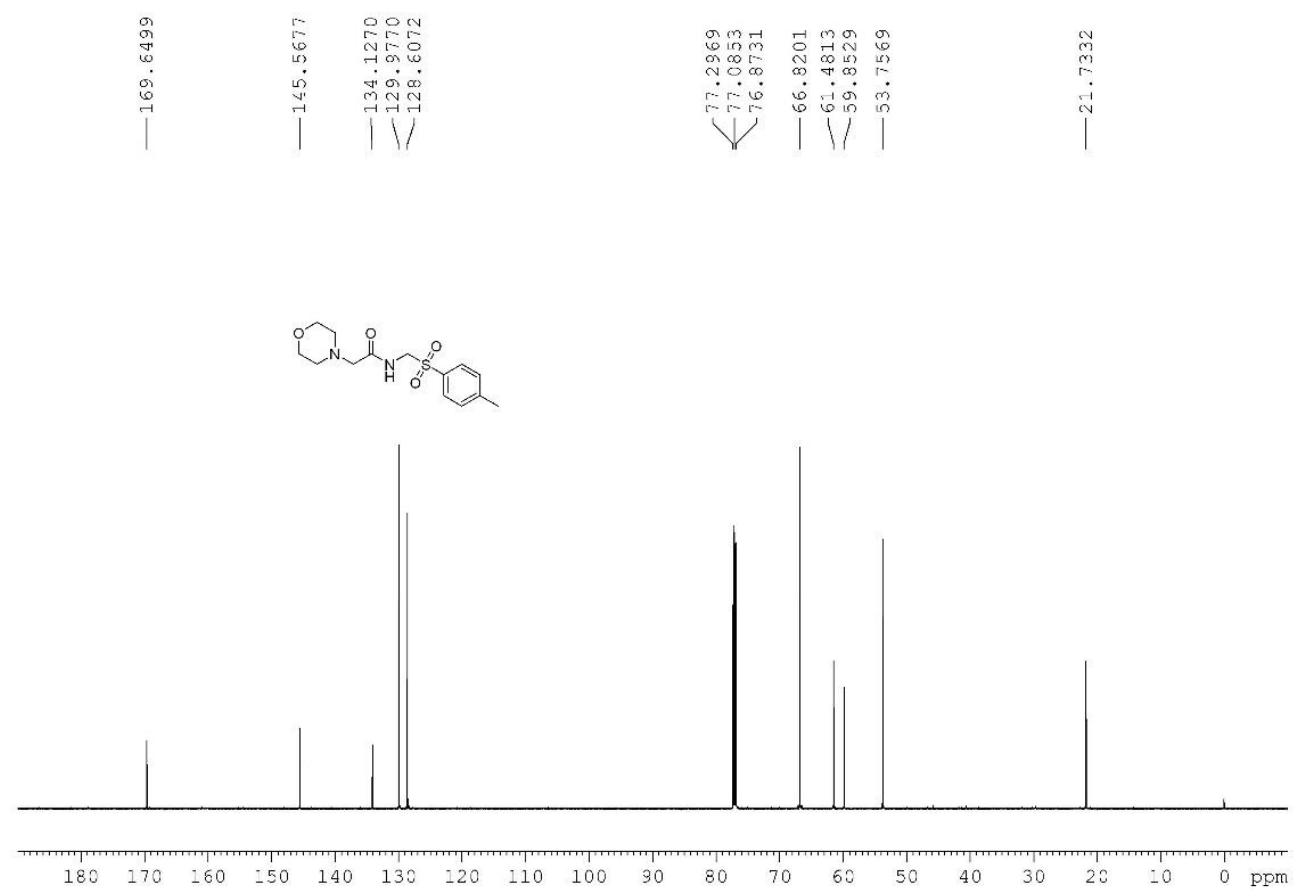
${ }^{1} \mathrm{H}$ NMR (600 MHz, $\mathrm{CDCl}_{3}$ ) of compound $\mathbf{5 m}$

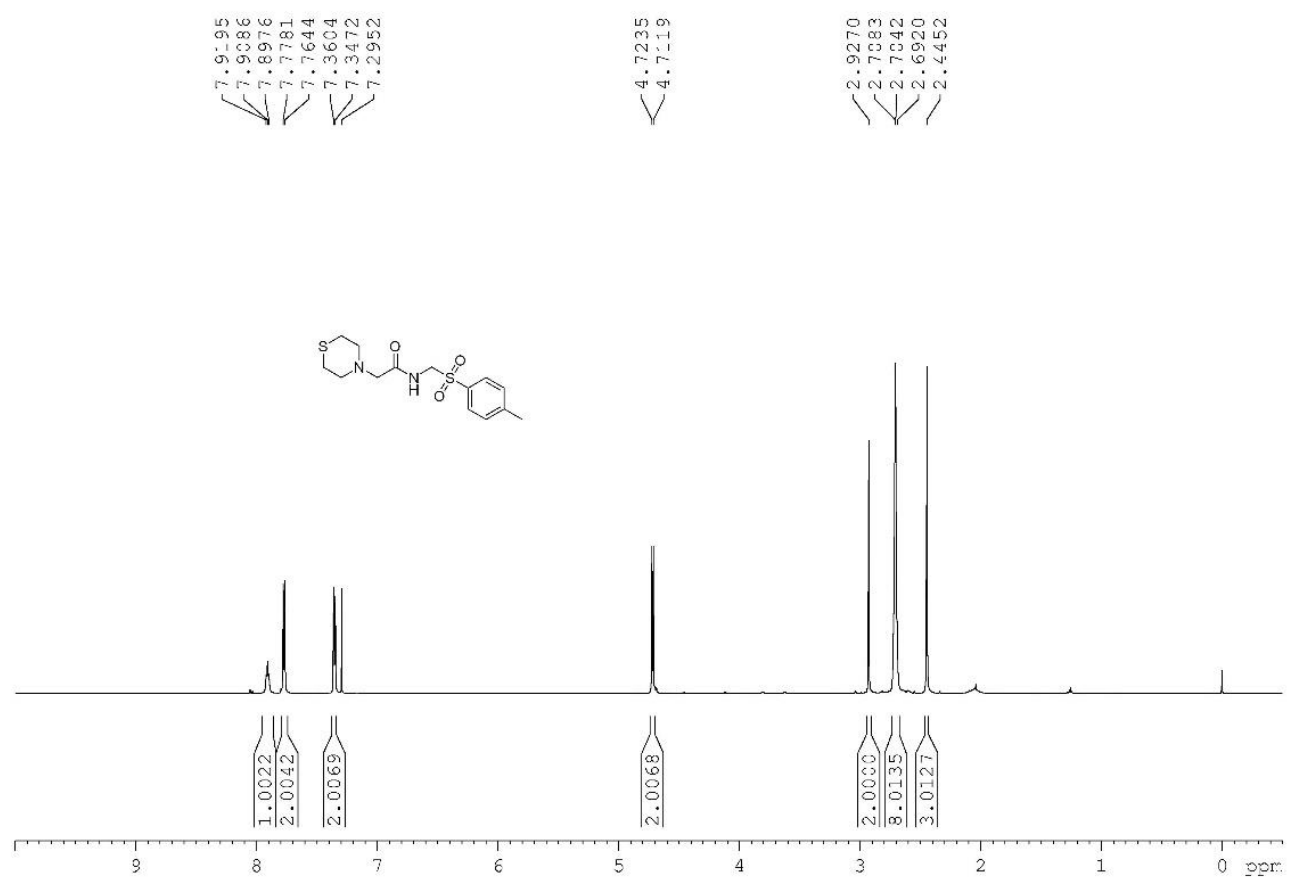

${ }^{13} \mathrm{C}\left\{{ }^{1} \mathrm{H}\right\}$ NMR (151 MHz, $\left.\mathrm{CDCl}_{3}\right)$ of compound $\mathbf{5 m}$

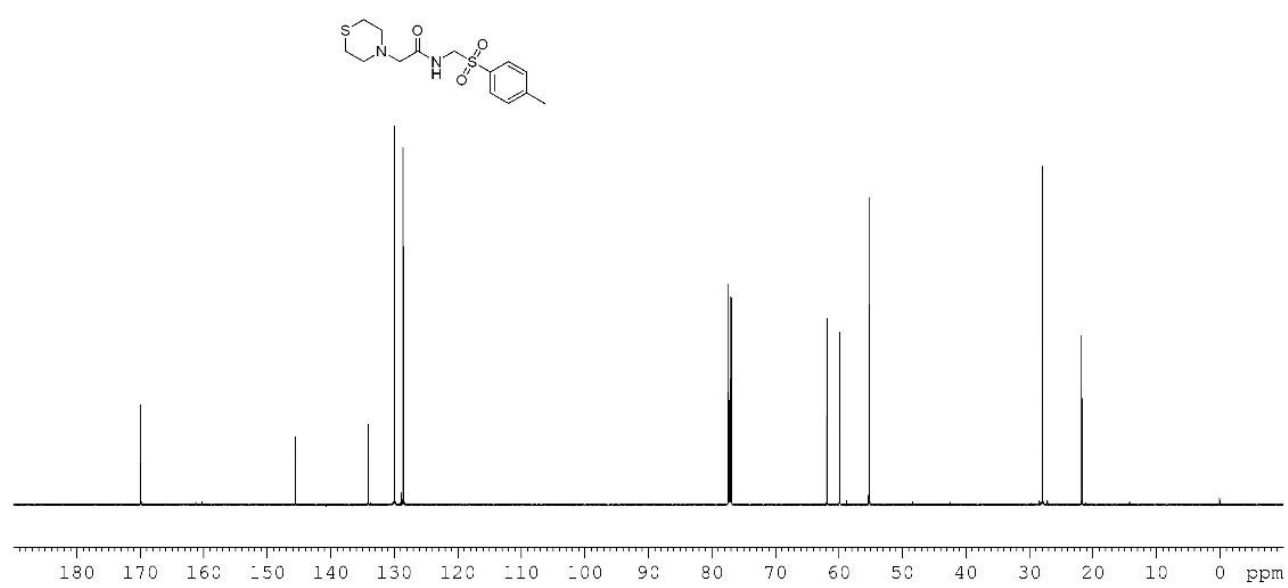


${ }^{1} \mathrm{H}$ NMR $\left(600 \mathrm{MHz}, \mathrm{CDCl}_{3}\right)$ of compound $\mathbf{5 n}$
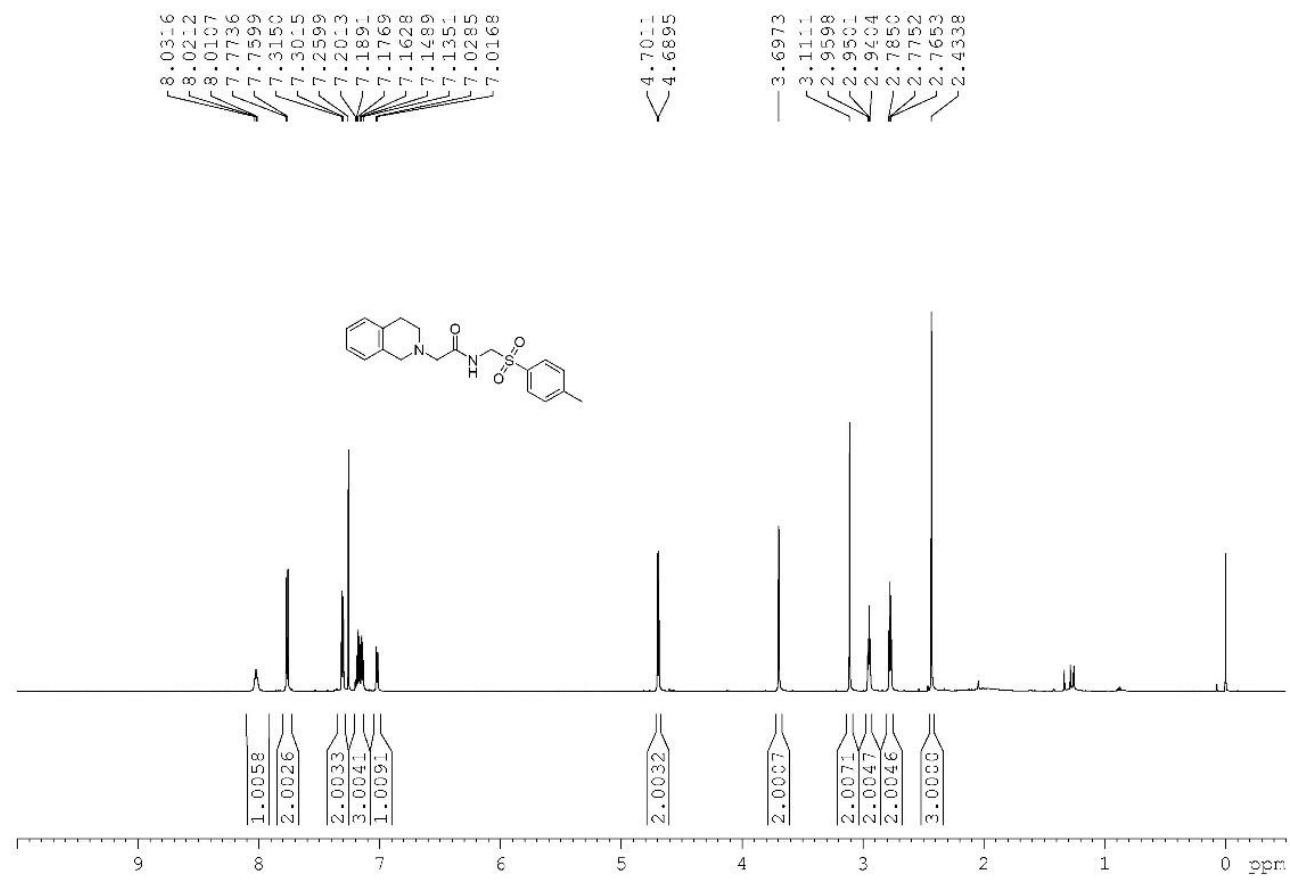

${ }^{13} \mathrm{C}\left\{{ }^{1} \mathrm{H}\right\}$ NMR (151 MHz, $\left.\mathrm{CDCl}_{3}\right)$ of compound $\mathbf{5 n}$

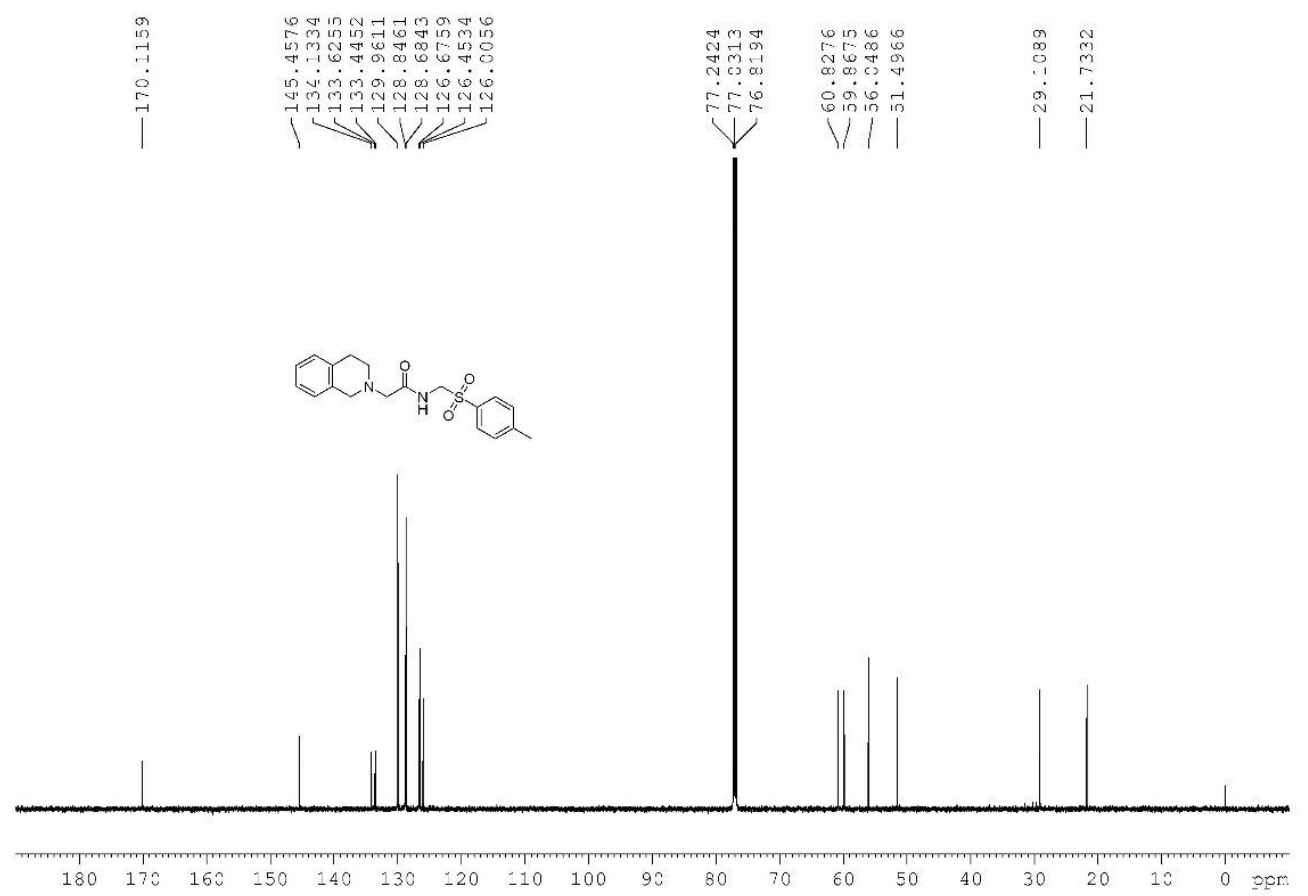


${ }^{1} \mathrm{H}$ NMR $\left(600 \mathrm{MHz}, \mathrm{CDCl}_{3}\right)$ of compound $\mathbf{6 a}$

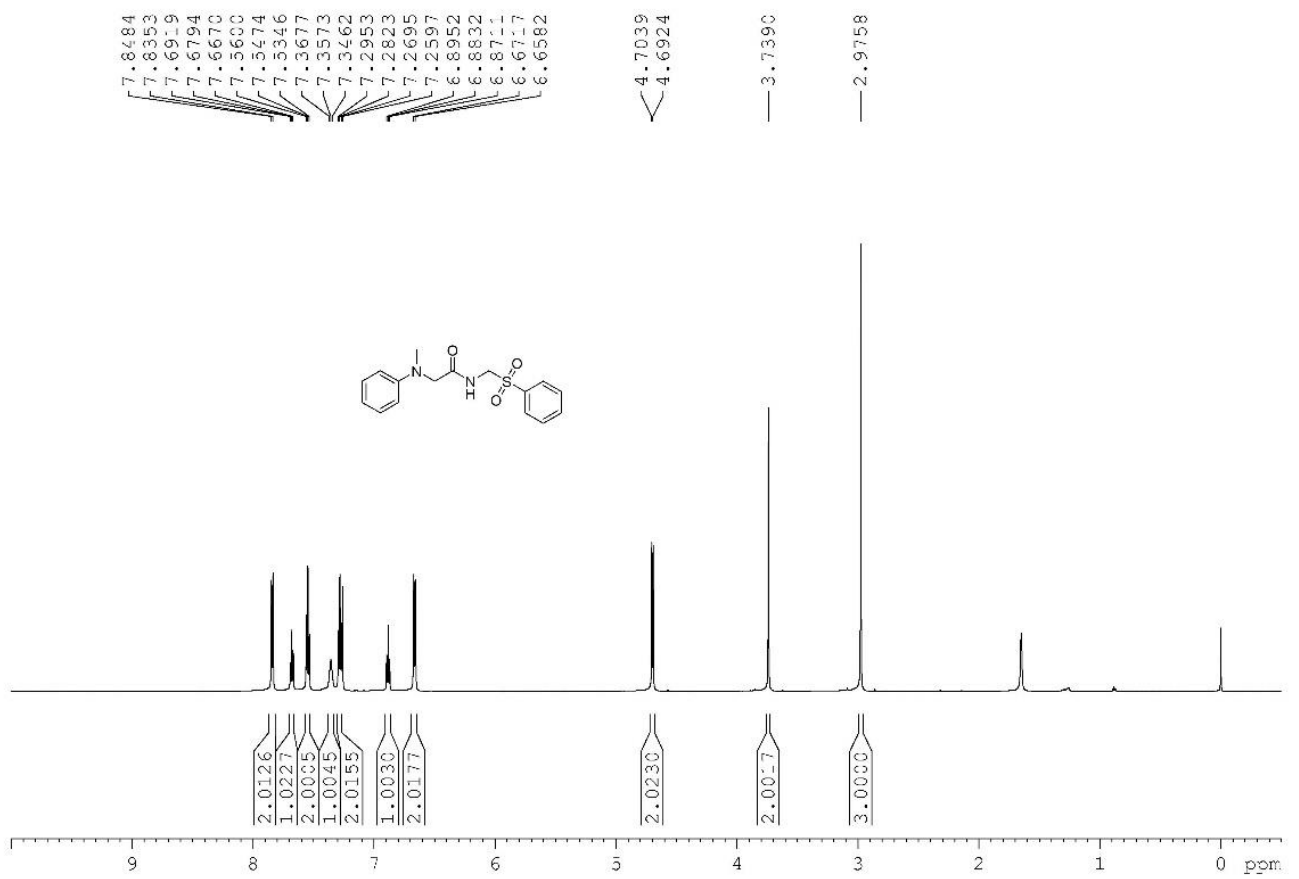

${ }^{13} \mathrm{C}\left\{{ }^{1} \mathrm{H}\right\}$ NMR (151 MHz, $\left.\mathrm{CDCl}_{3}\right)$ of compound $\mathbf{6 a}$

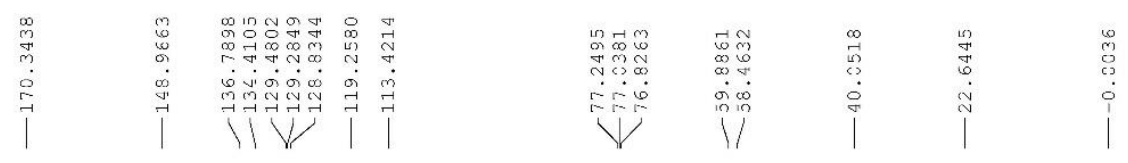

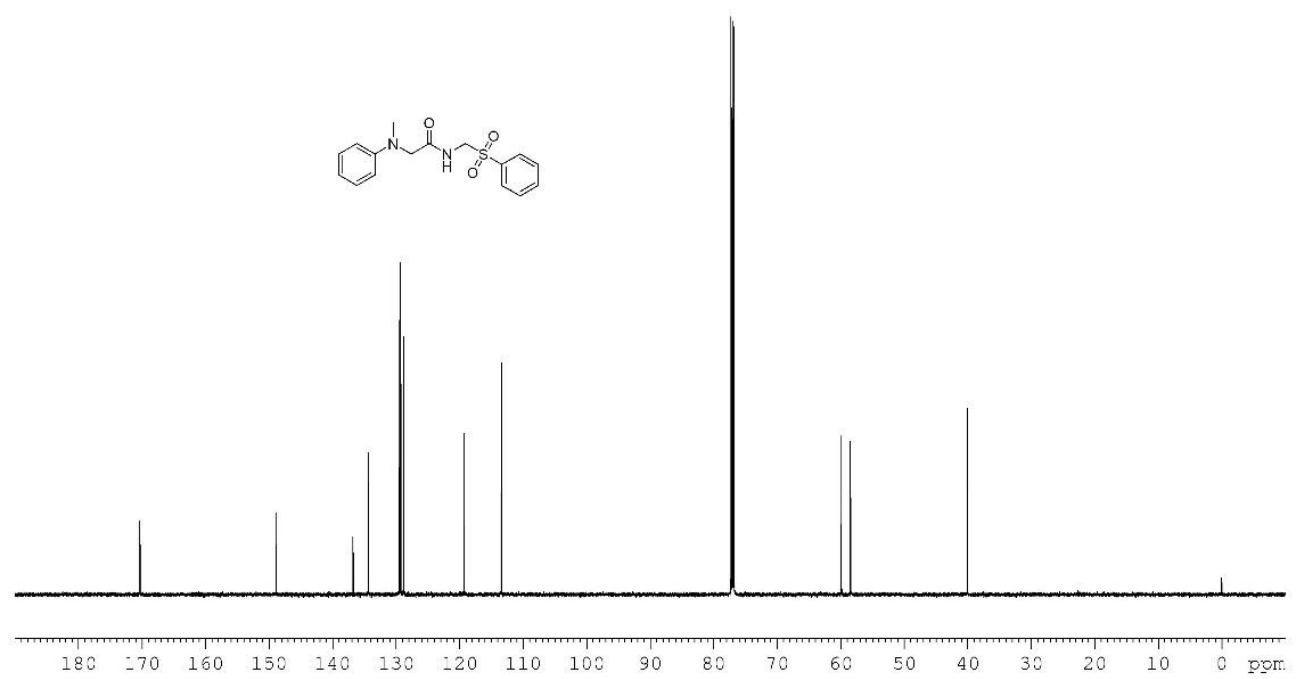


${ }^{1} \mathrm{H}$ NMR $\left(600 \mathrm{MHz}, \mathrm{CDCl}_{3}\right)$ of compound $\mathbf{6 b}$

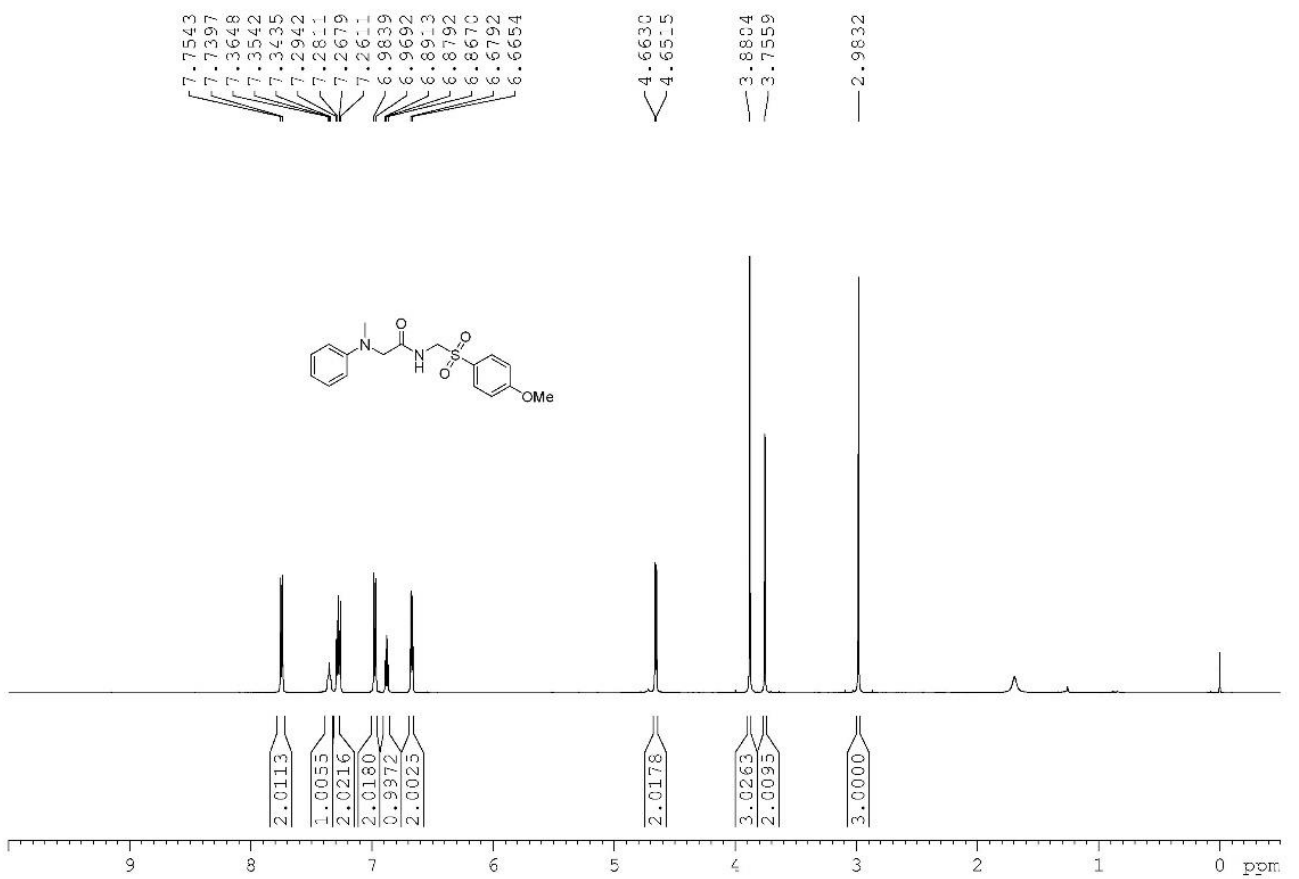

${ }^{13} \mathrm{C}\left\{{ }^{1} \mathrm{H}\right\}$ NMR (151 MHz, $\mathrm{CDCl}_{3}$ ) of compound $\mathbf{6 b}$
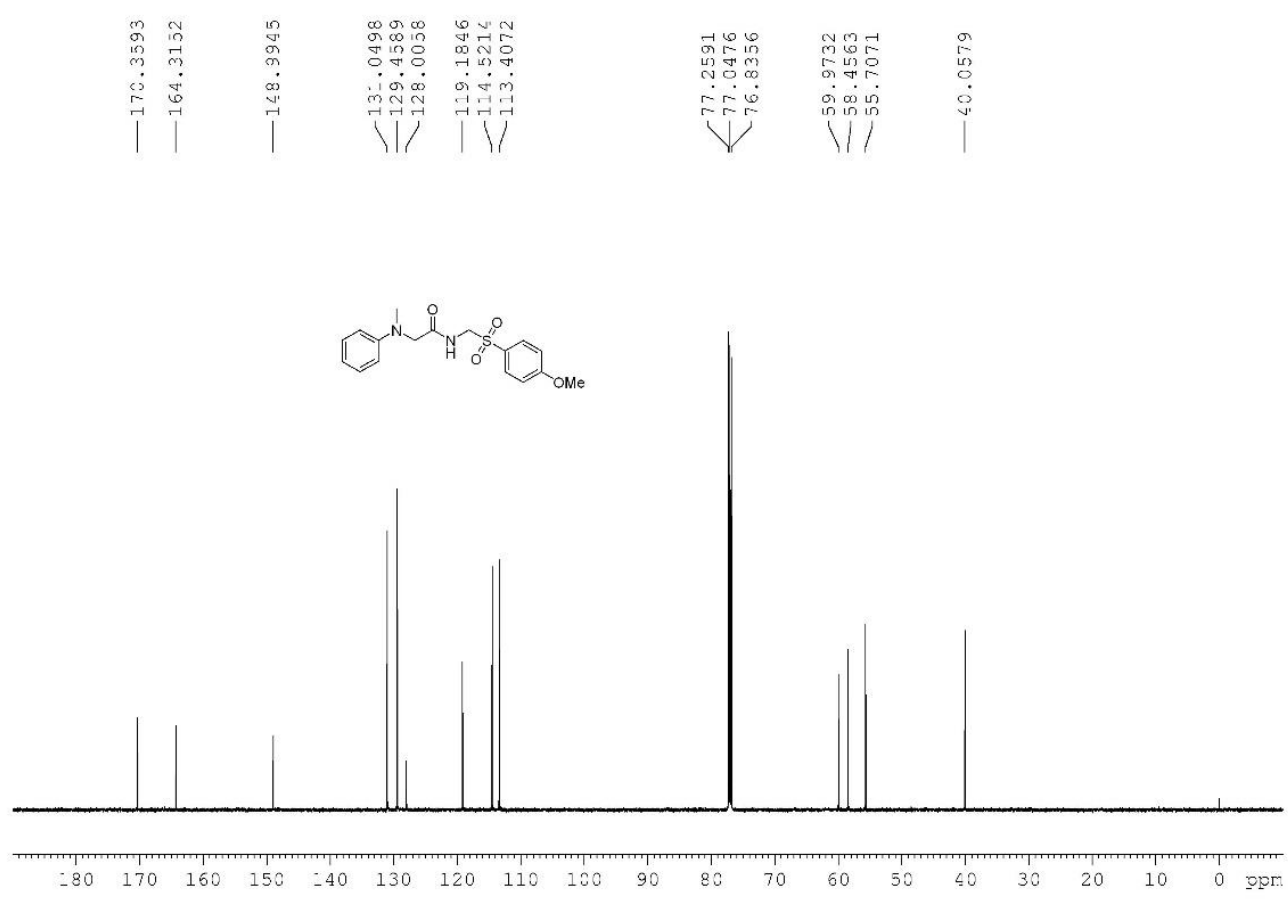
${ }^{1} \mathrm{H}$ NMR $\left(600 \mathrm{MHz}, \mathrm{CDCl}_{3}\right)$ of compound $\mathbf{6 c}$

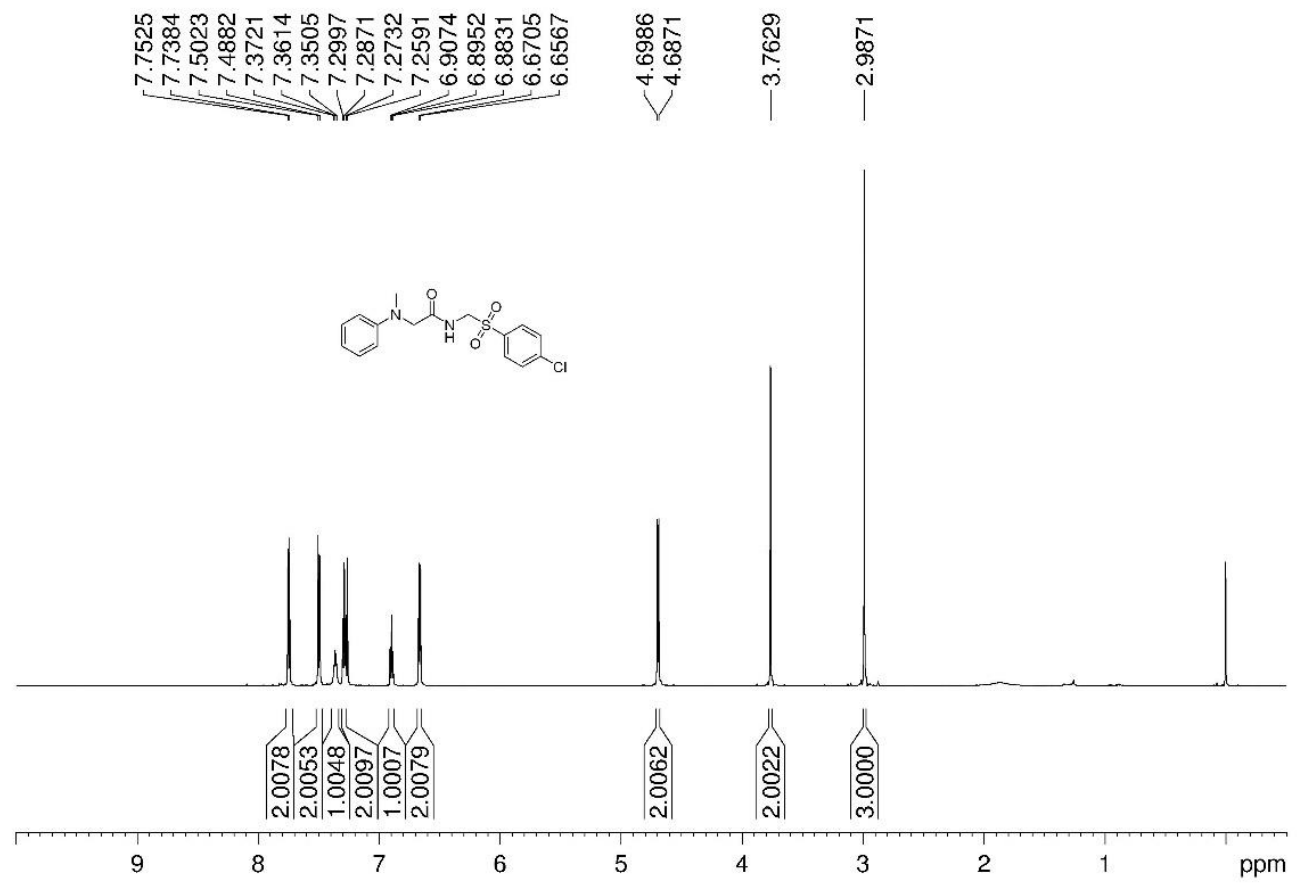

${ }^{13} \mathrm{C}\left\{{ }^{1} \mathrm{H}\right\}$ NMR (151 MHz, $\left.\mathrm{CDCl} 3\right)$ of compound $\mathbf{6 c}$
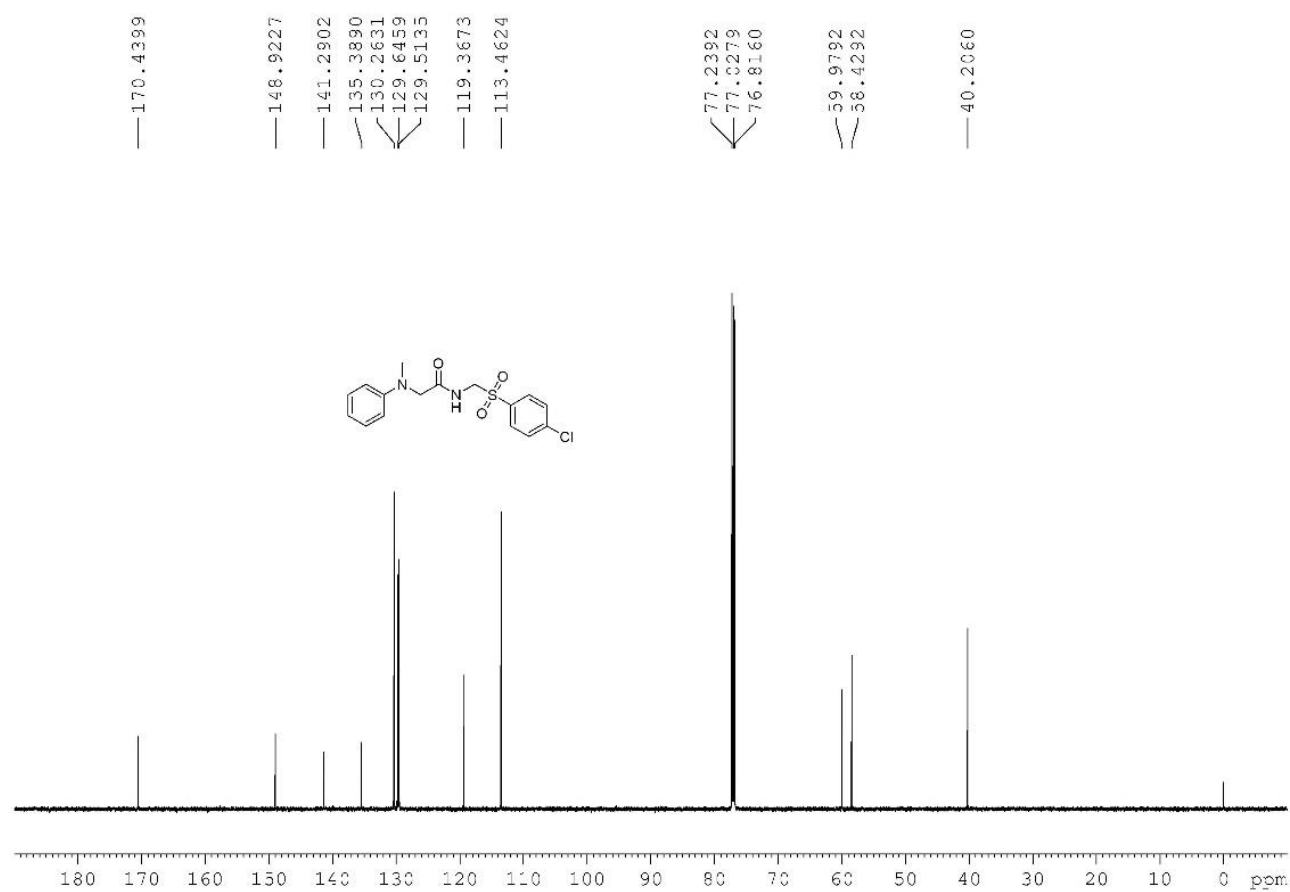
${ }^{1} \mathrm{H}$ NMR (600 MHz, $\mathrm{CDCl}_{3}$ ) of compound $\mathbf{6 d}$
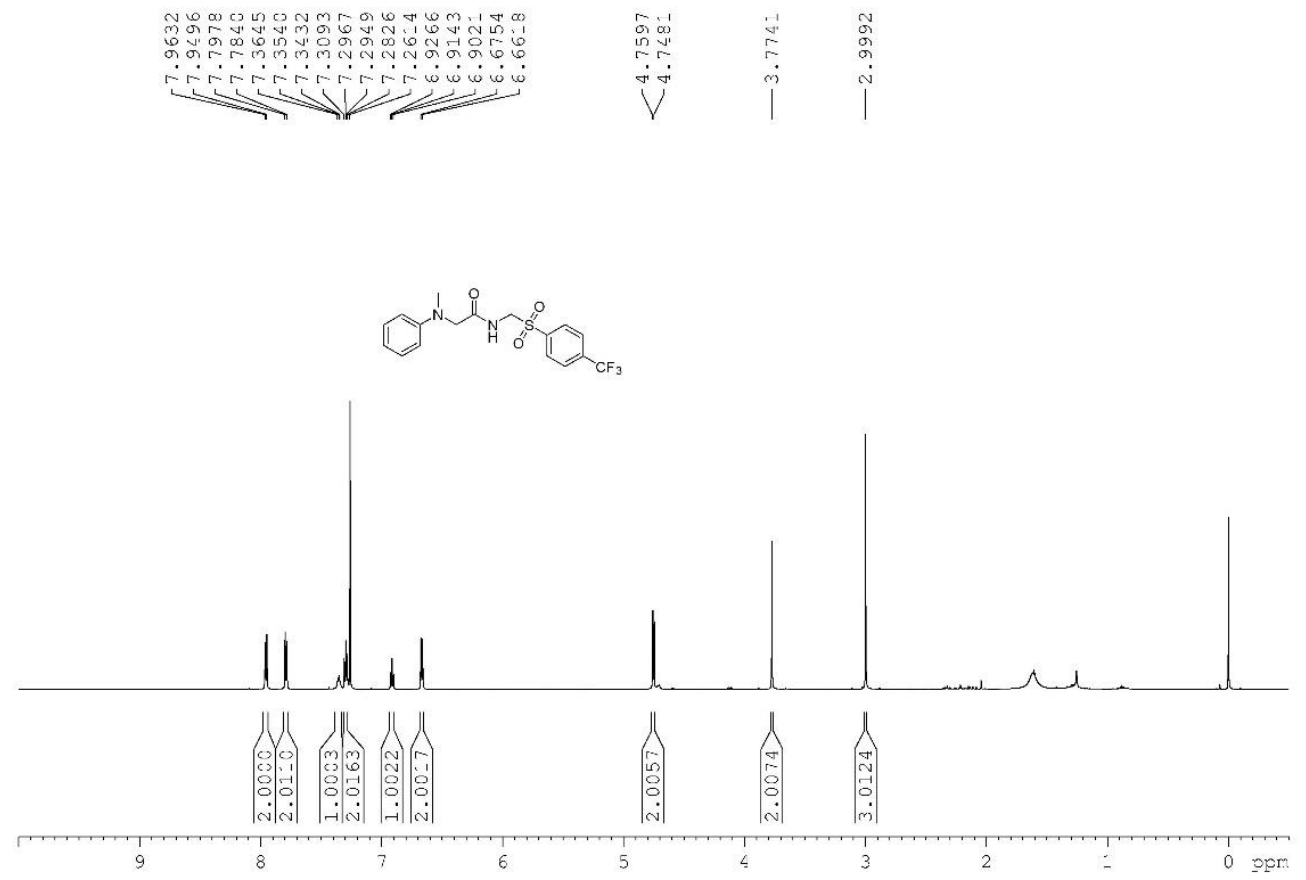

${ }^{13} \mathrm{C}\left\{{ }^{1} \mathrm{H}\right\}$ NMR (151 MHz, $\left.\mathrm{CDCl}_{3}\right)$ of compound $\mathbf{6 d}$

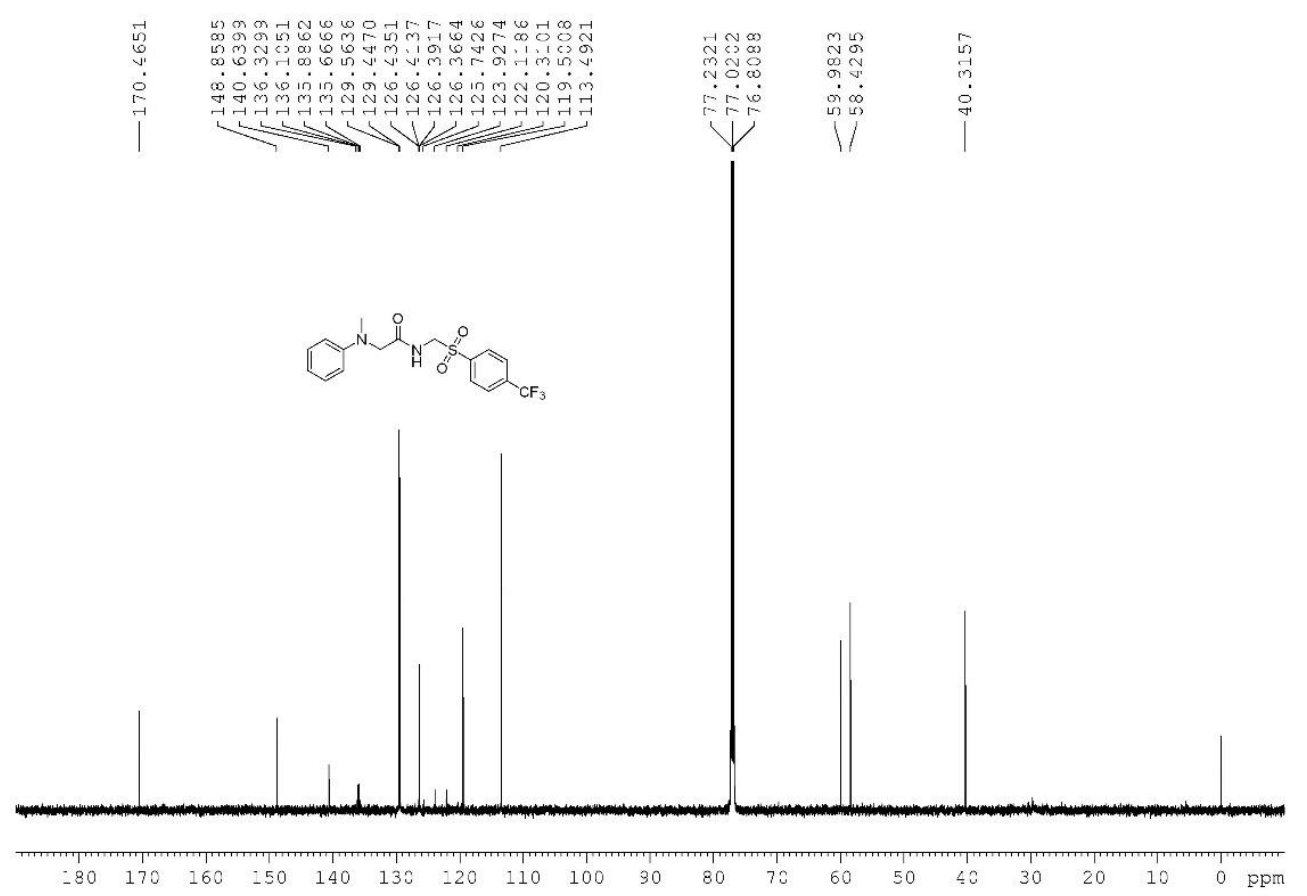


${ }^{19} \mathrm{~F}$ NMR (565 MHz, $\mathrm{CDCl}_{3}$ ) of compound $\mathbf{6 d}$

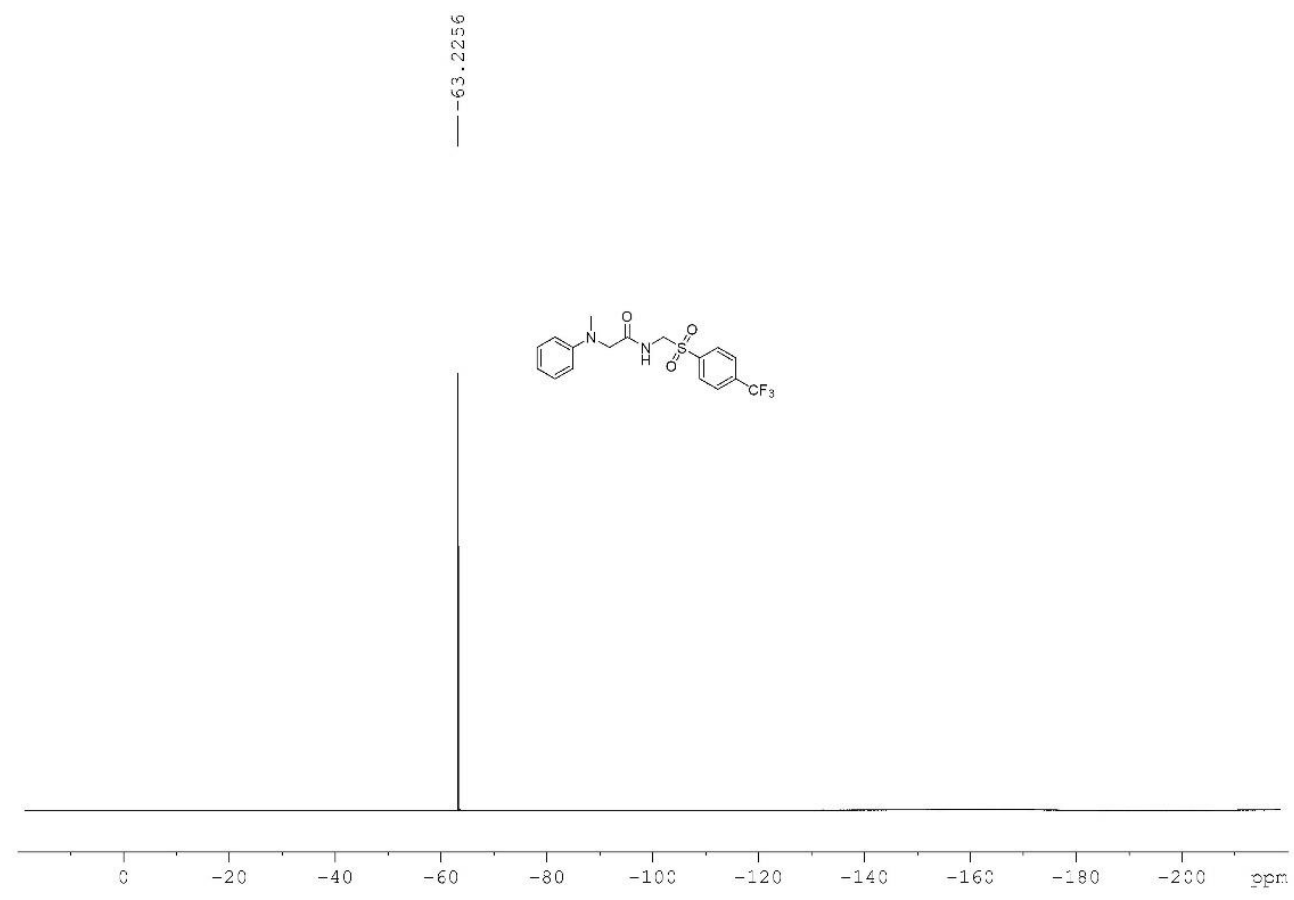

${ }^{1} \mathrm{H}$ NMR (600 MHz, $\left.\mathrm{CDCl}_{3}\right)$ of compound $\mathbf{6 e}$
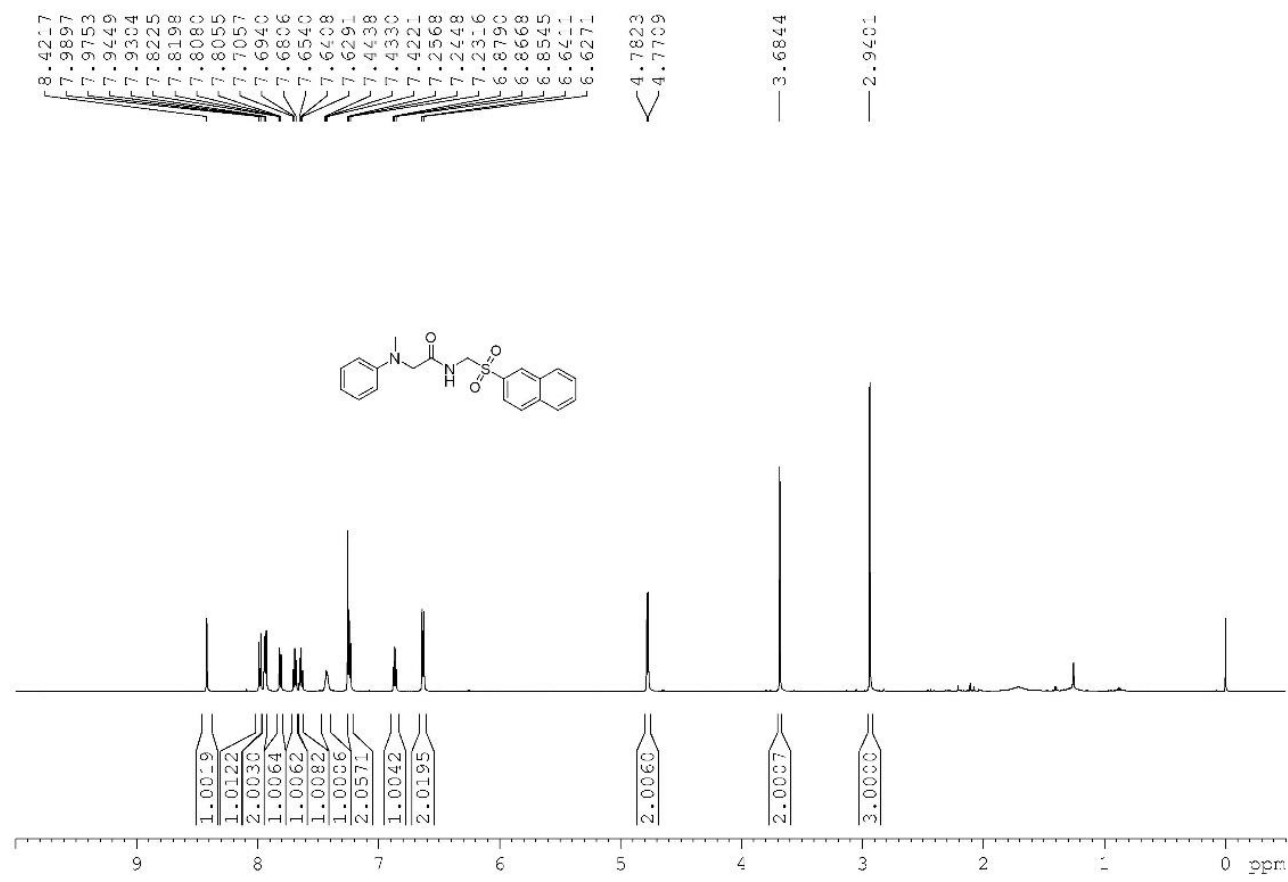
${ }^{13} \mathrm{C}\left\{{ }^{1} \mathrm{H}\right\}$ NMR (151 MHz, $\left.\mathrm{CDCl}_{3}\right)$ of compound $\mathbf{6 e}$
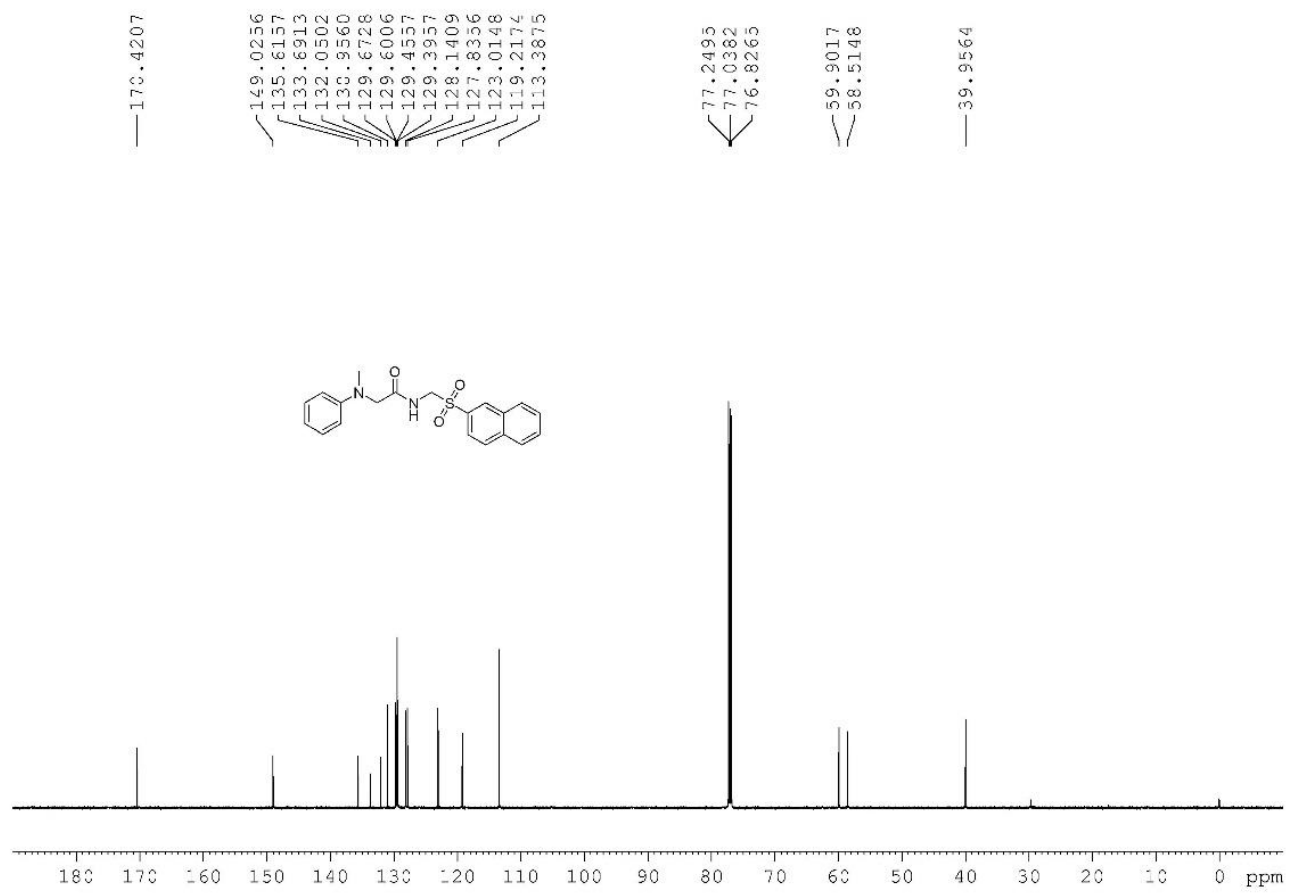

${ }^{1} \mathrm{H}$ NMR $\left(600 \mathrm{MHz}, \mathrm{CDCl}_{3}\right)$ of compound $\mathbf{6 f}$
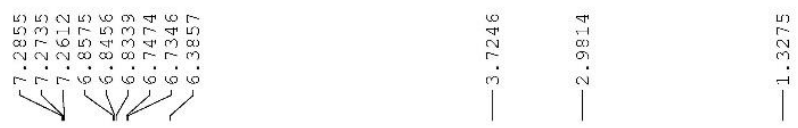

$\mathbb{1}^{i} \operatorname{lin}_{n} x$

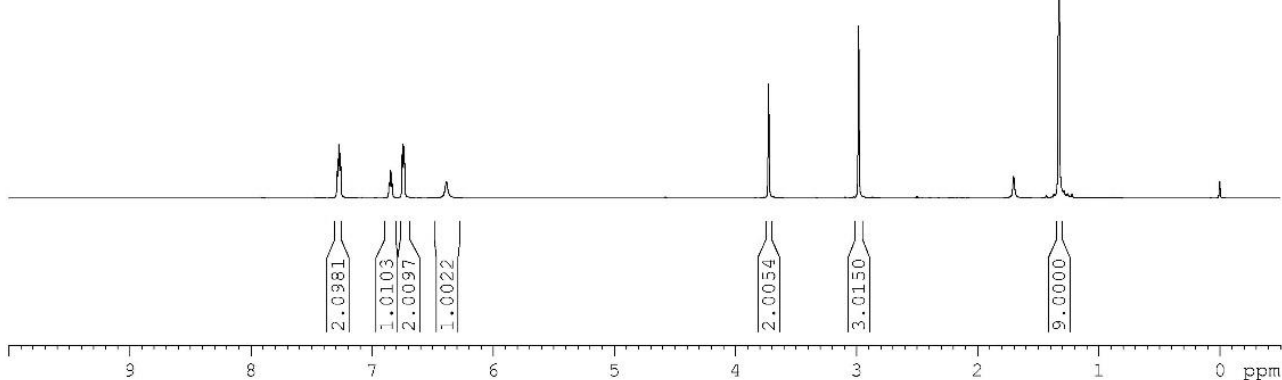


${ }^{13} \mathrm{C}\left\{{ }^{1} \mathrm{H}\right\}$ NMR (151 MHz, $\left.\mathrm{CDCl}_{3}\right)$ of compound $\mathbf{6 f}$

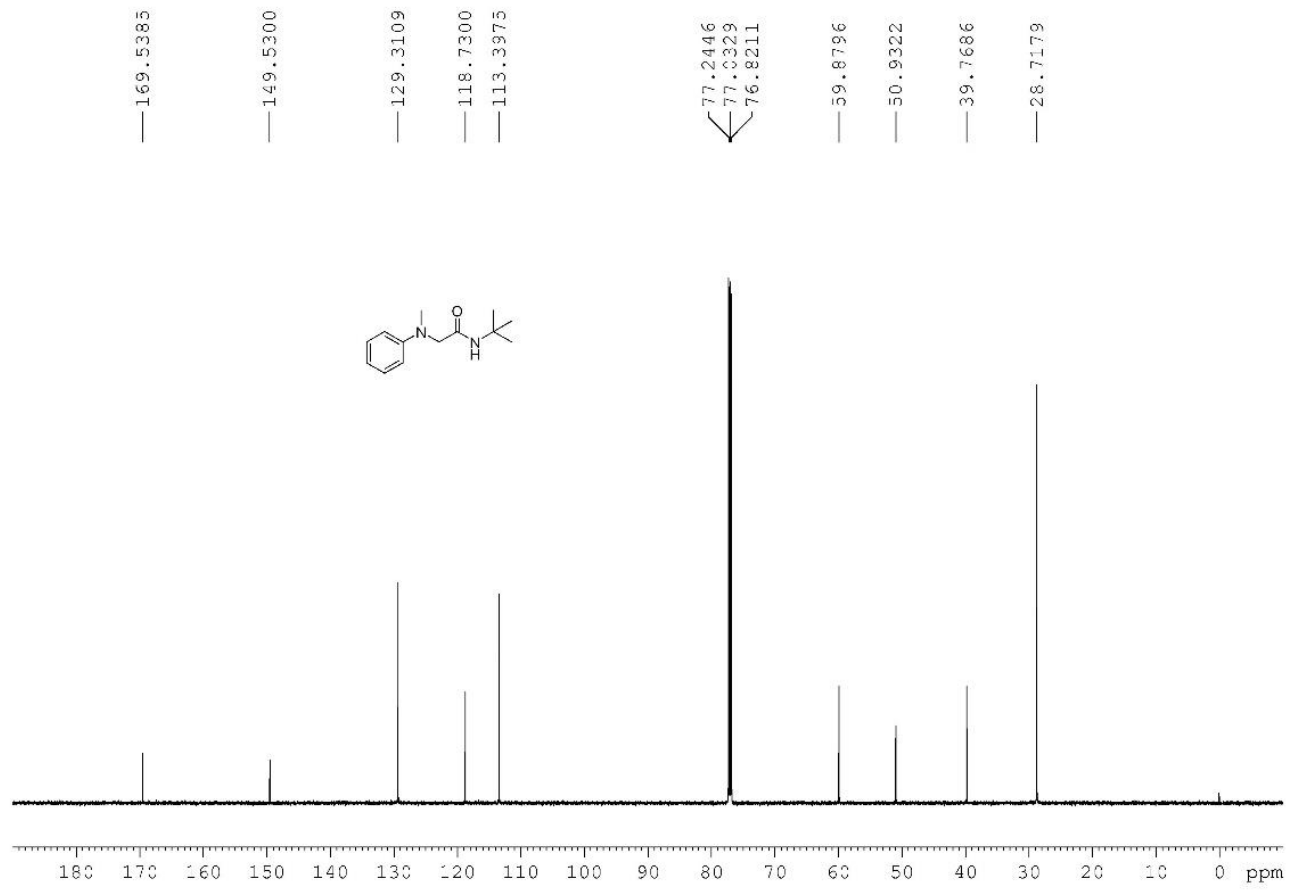

${ }^{1} \mathrm{H}$ NMR (600 MHz, $\left.\mathrm{CDCl}_{3}\right)$ of compound $\mathbf{6 g}$
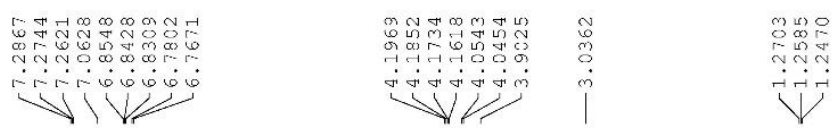

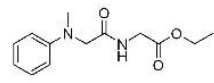

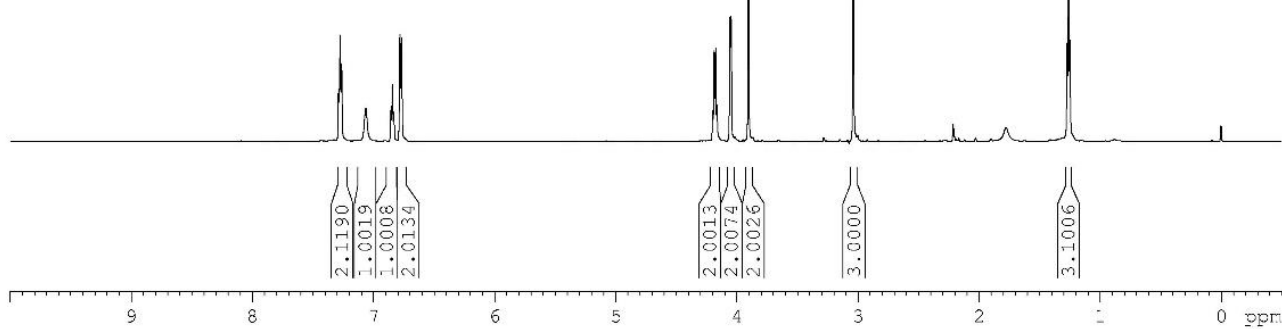


${ }^{13} \mathrm{C}\left\{{ }^{1} \mathrm{H}\right\}$ NMR (151 MHz, $\mathrm{CDCl}_{3}$ ) of compound $\mathbf{6} \mathbf{h}$

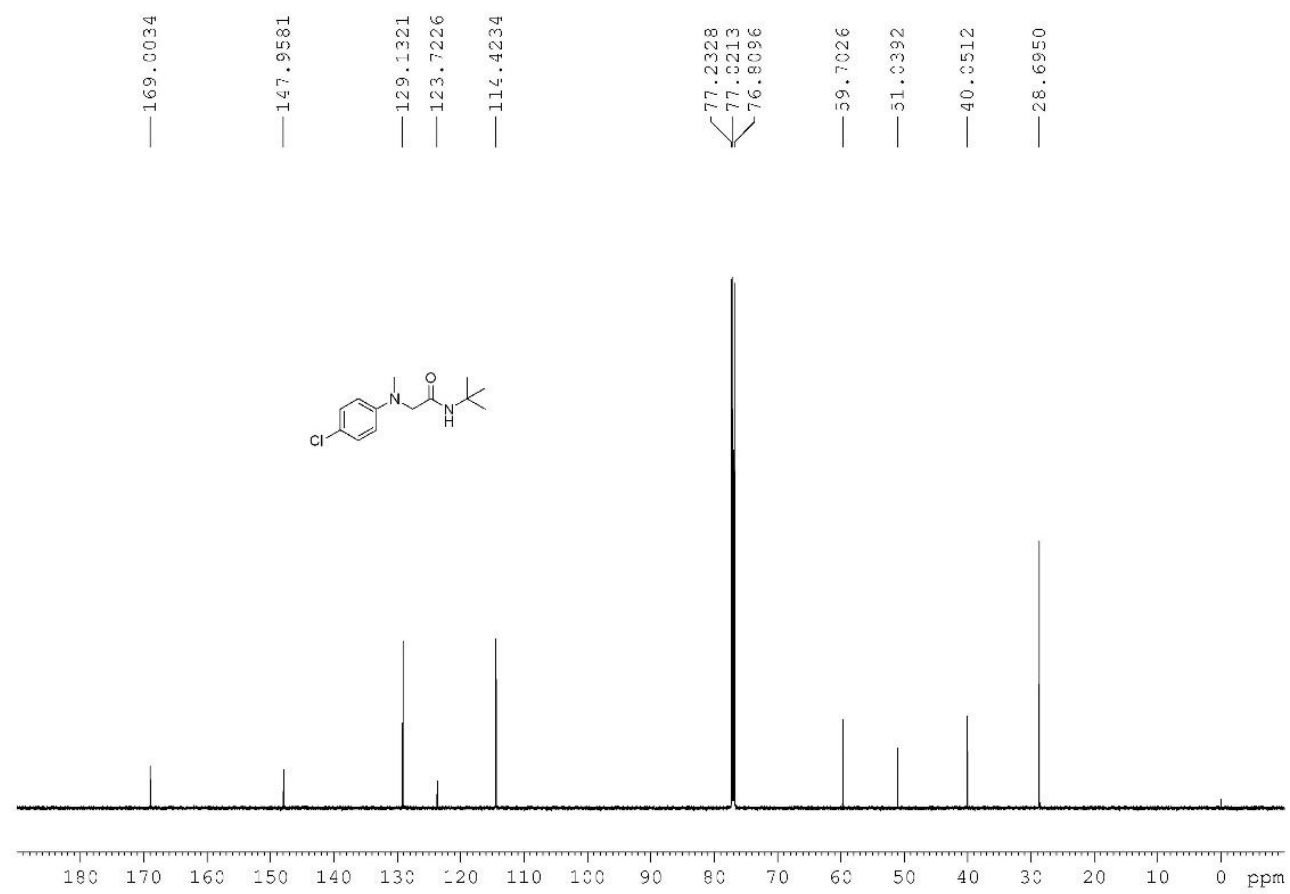

${ }^{1} \mathrm{H}$ NMR $\left(600 \mathrm{MHz}, \mathrm{CDCl}_{3}\right)$ of compound $\mathbf{6 i}$
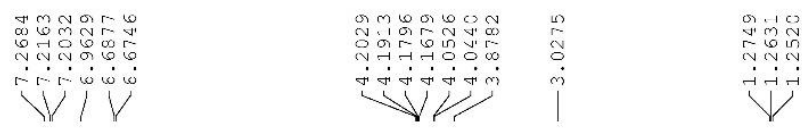

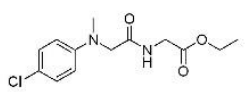

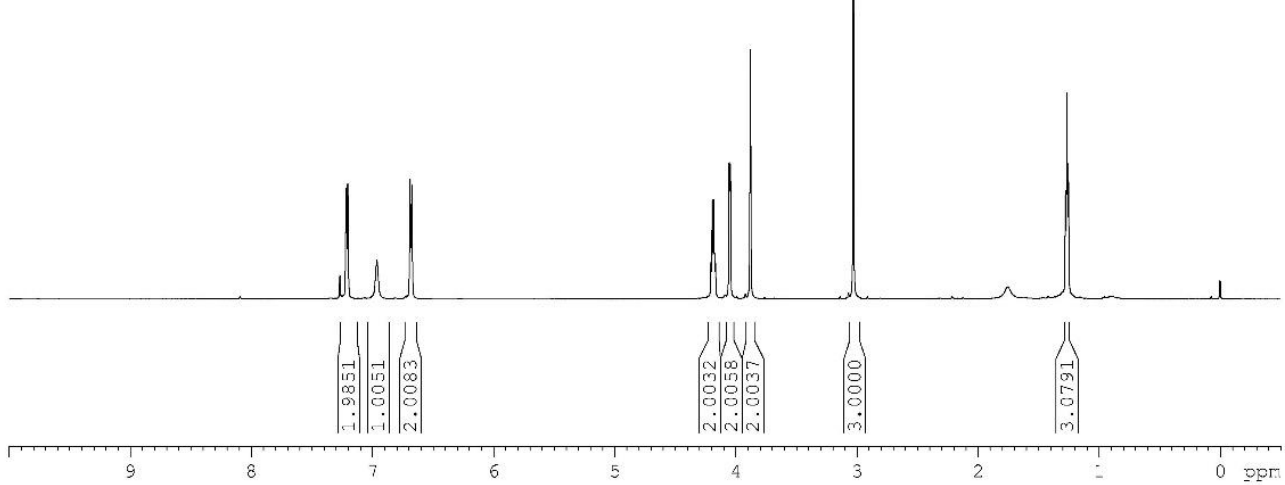


${ }^{13} \mathrm{C}\left\{{ }^{1} \mathrm{H}\right\}$ NMR (151 MHz, $\mathrm{CDCl}_{3}$ ) of compound $\mathbf{6 i}$

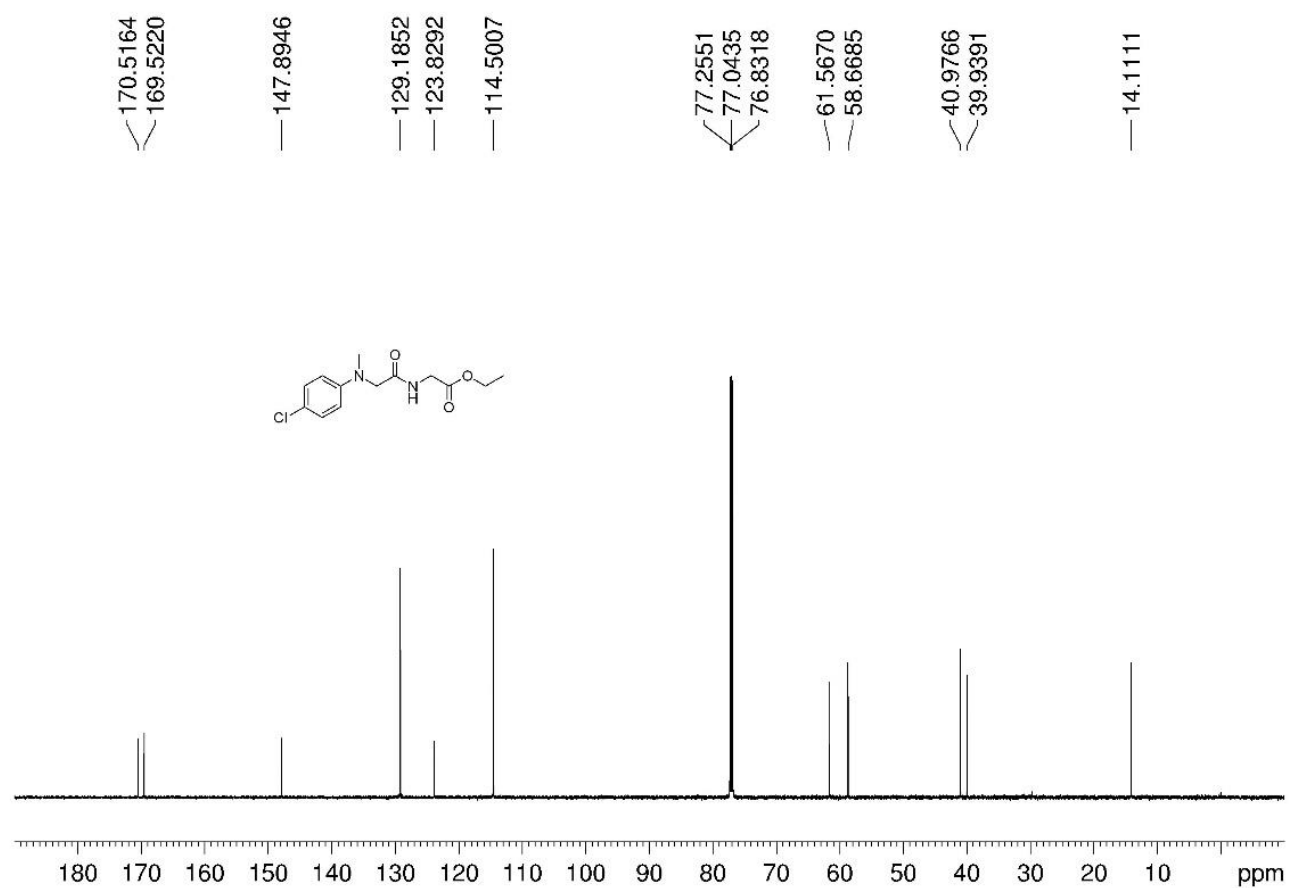

${ }^{1} \mathrm{H}$ NMR (600 MHz, $\mathrm{CDCl}_{3}$ ) of compound 7

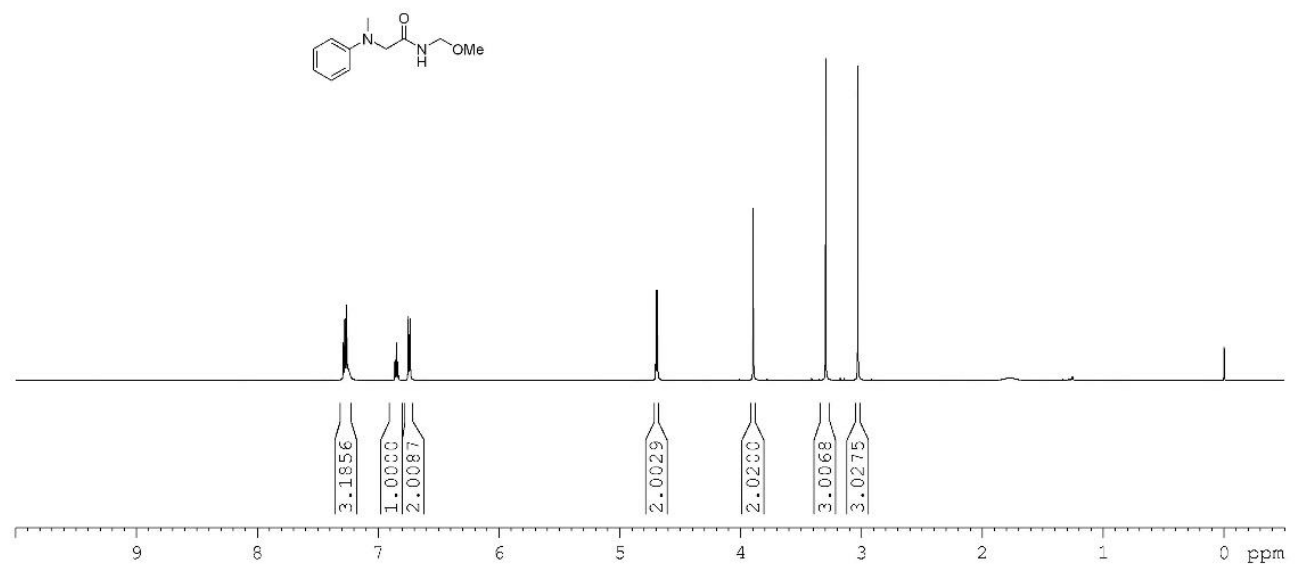


${ }^{13} \mathrm{C}\left\{{ }^{1} \mathrm{H}\right\}$ NMR (151 MHz, $\mathrm{CDCl}_{3}$ ) of compound 7

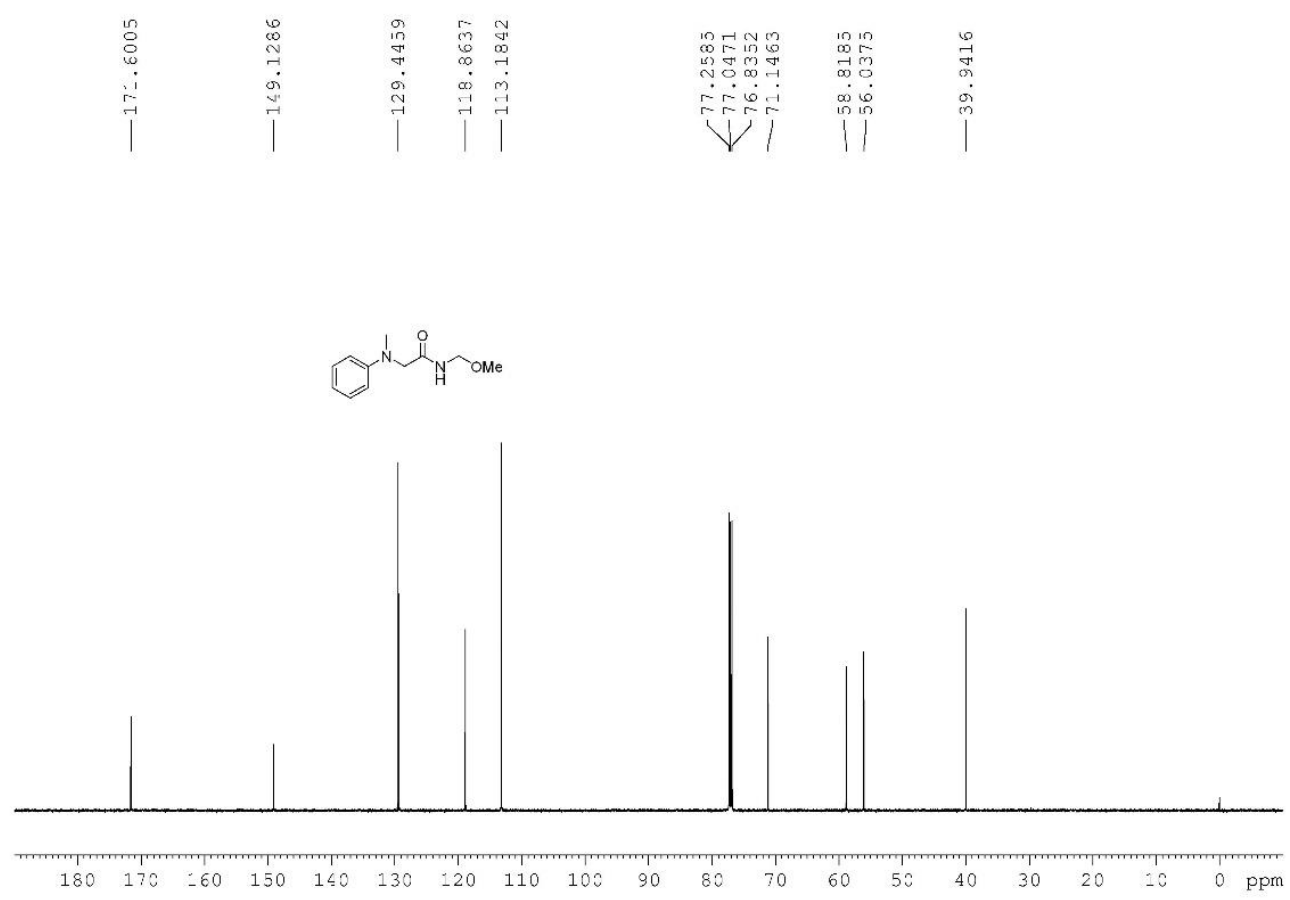

${ }^{1} \mathrm{H}$ NMR (600 MHz, $\mathrm{CDCl}_{3}$ ) of compound 8

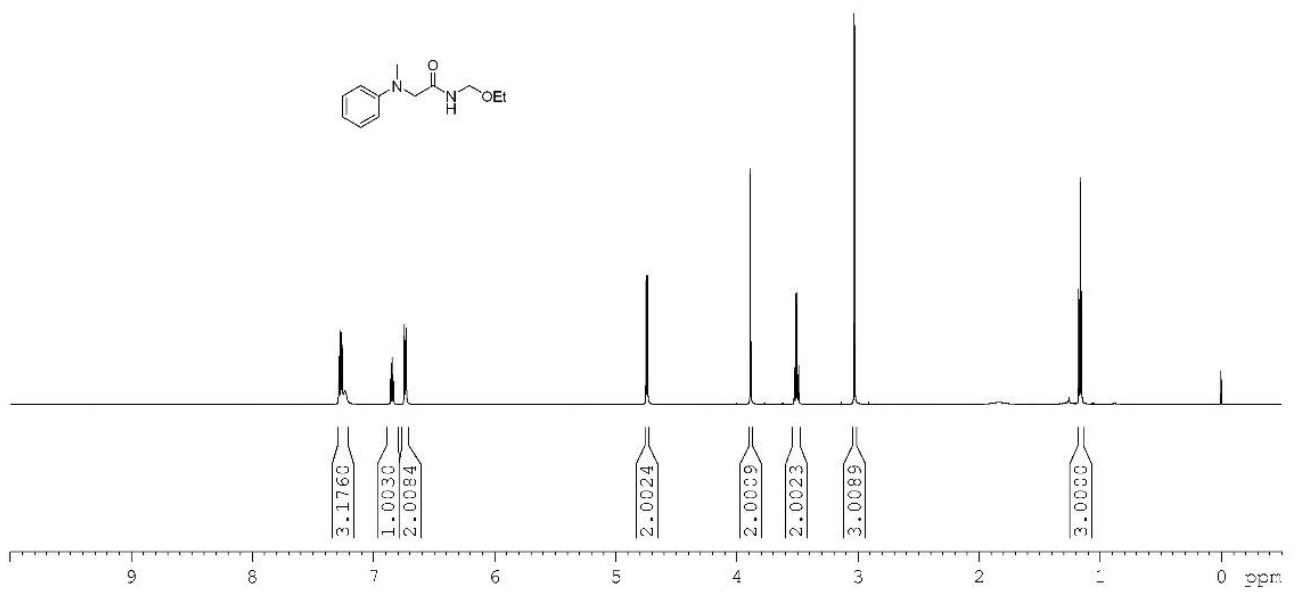


${ }^{13} \mathrm{C}\left\{{ }^{1} \mathrm{H}\right\}$ NMR (151 MHz, $\left.\mathrm{CDCl}_{3}\right)$ of compound $\mathbf{8}$

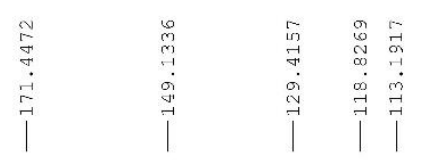

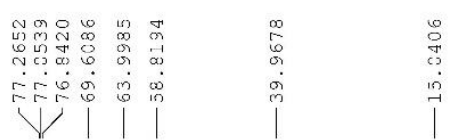

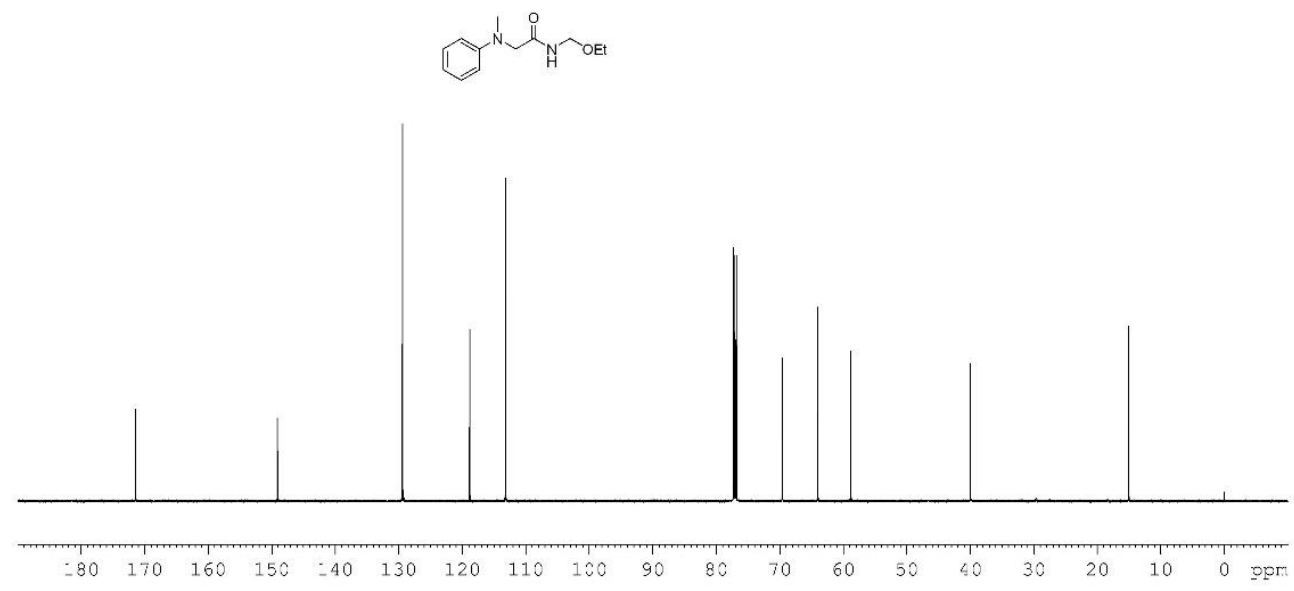

${ }^{1} \mathrm{H}$ NMR (600 MHz, $\mathrm{CDCl}_{3}$ ) of compound 9

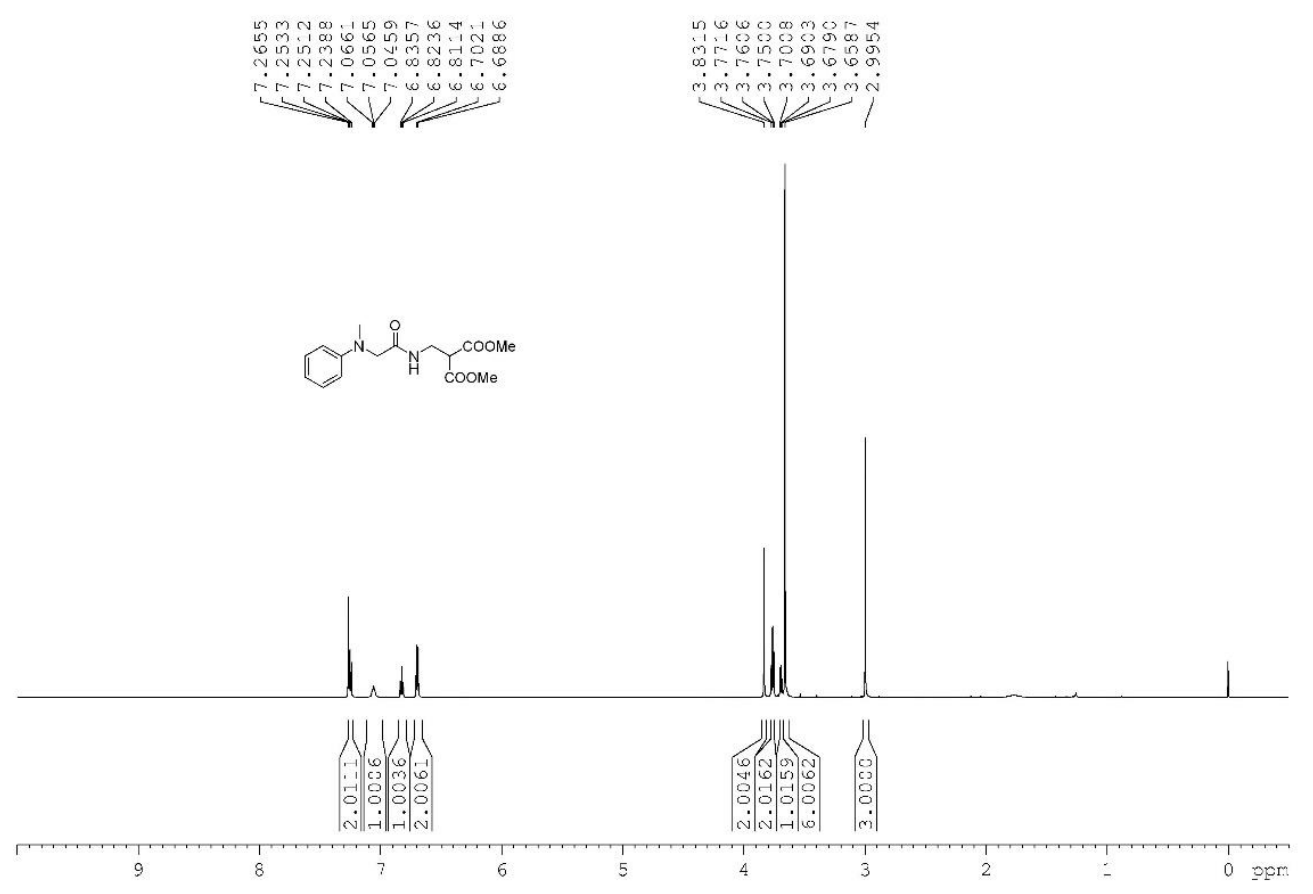


${ }^{13} \mathrm{C}\left\{{ }^{1} \mathrm{H}\right\}$ NMR (151 MHz, $\left.\mathrm{CDCl}_{3}\right)$ of compound 9

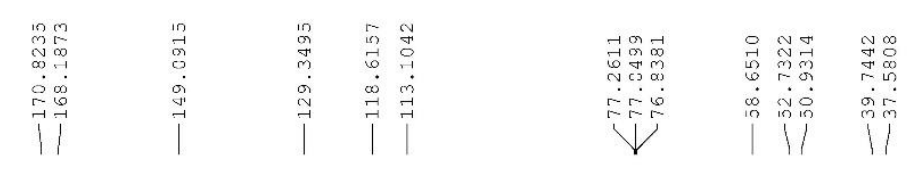

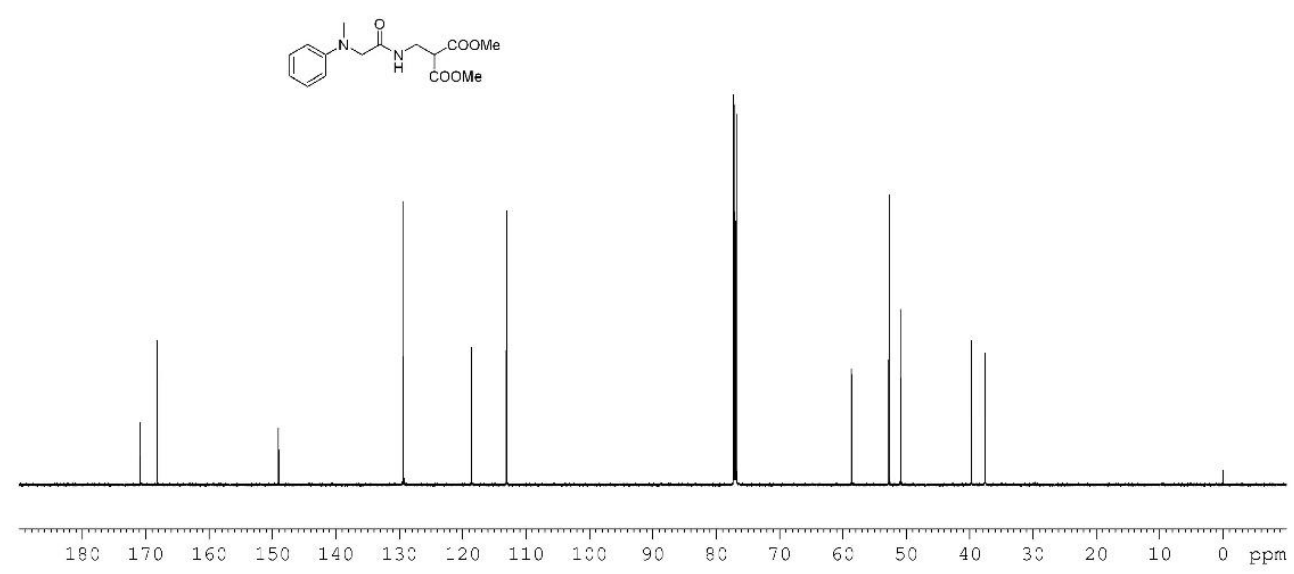

${ }^{1} \mathrm{H}$ NMR (600 MHz, $\mathrm{CDCl}_{3}$ ) of compound $\mathbf{1 0}$

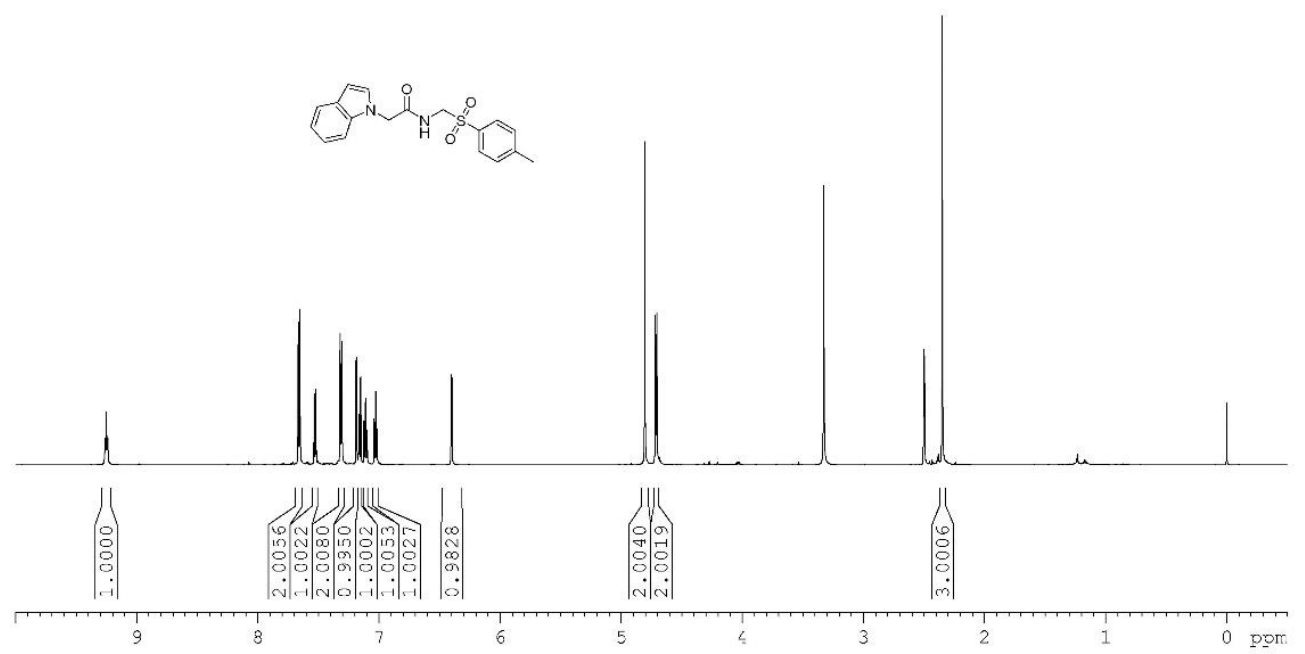


${ }^{13} \mathrm{C}\left\{{ }^{1} \mathrm{H}\right\}$ NMR (151 MHz, $\left.\mathrm{CDCl}_{3}\right)$ of compound $\mathbf{1 0}$

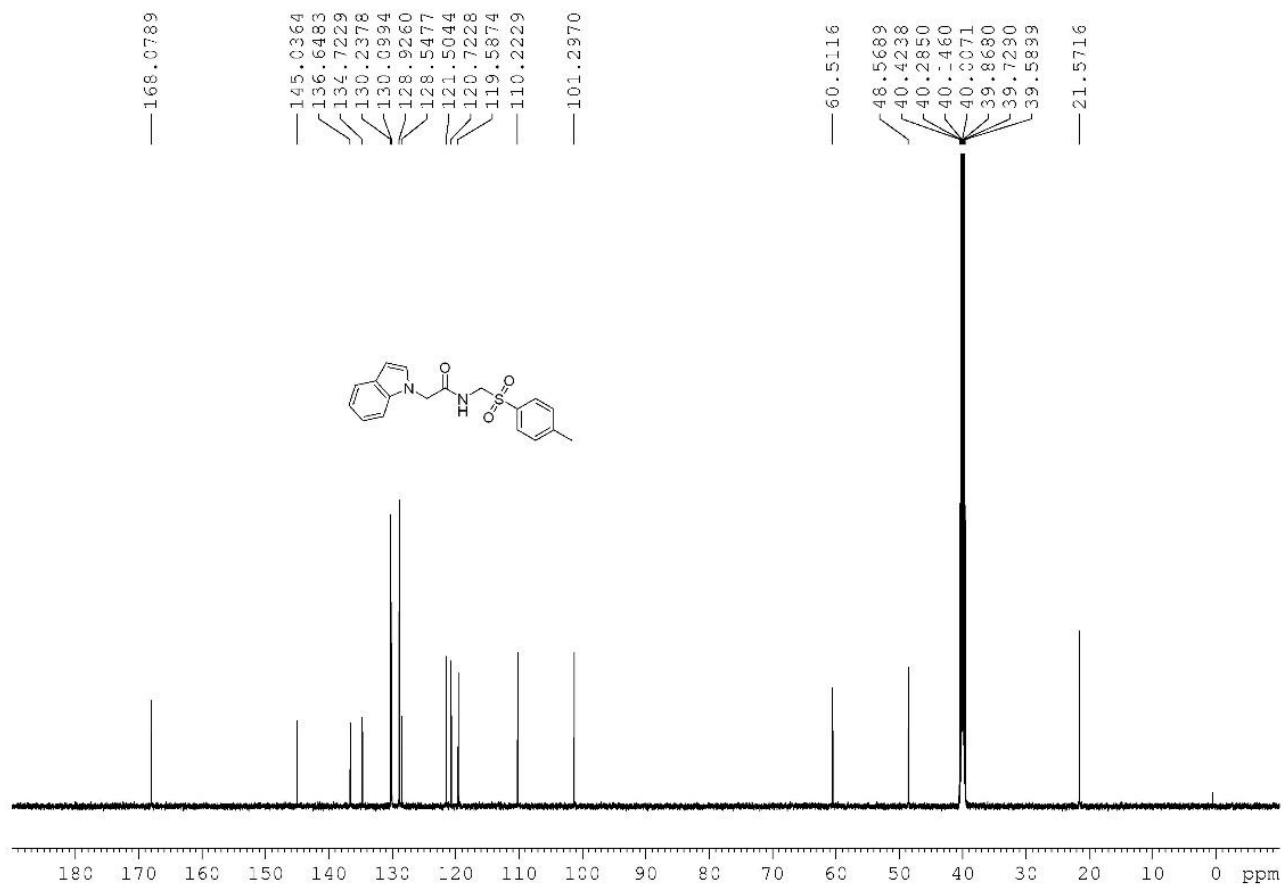

${ }^{1} \mathrm{H} \mathrm{NMR}\left(600 \mathrm{MHz}, \mathrm{CDCl}_{3}\right)$ of compound 11a
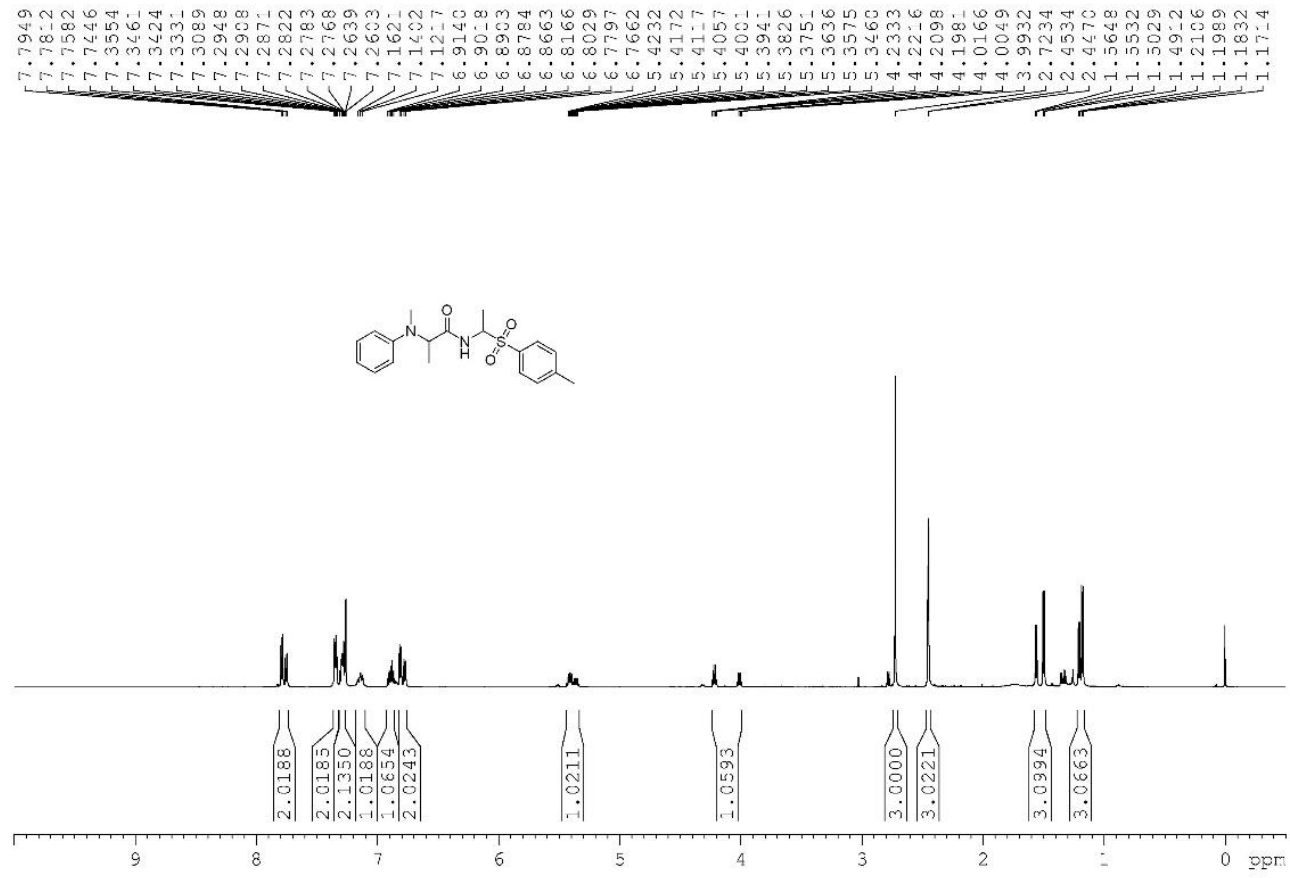
${ }^{13} \mathrm{C}\left\{{ }^{1} \mathrm{H}\right\}$ NMR $\left(151 \mathrm{MHz}, \mathrm{CDCl}_{3}\right)$ of compound 11a

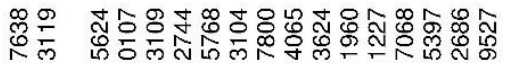

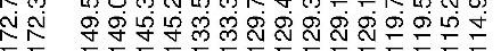

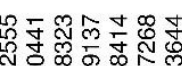

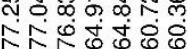

NiV
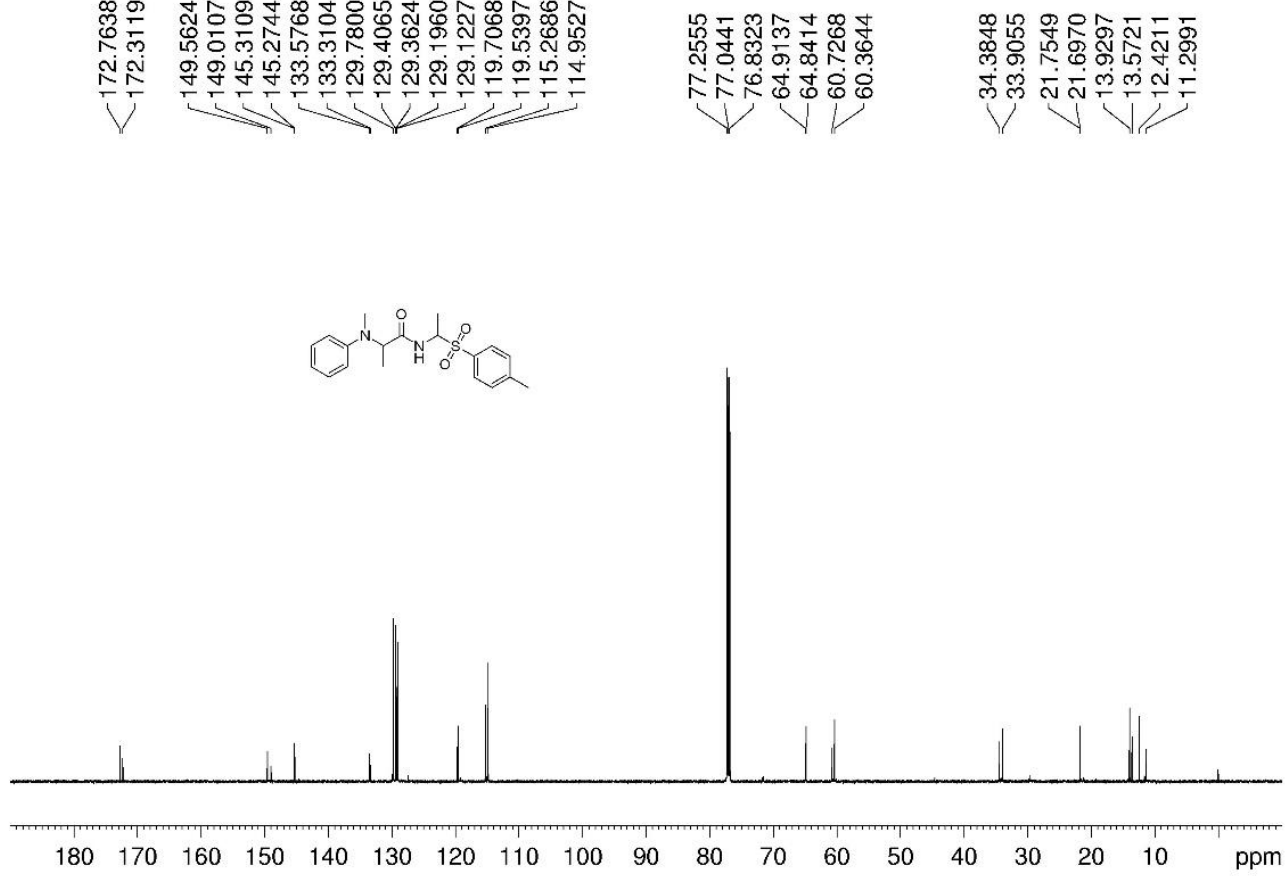

${ }^{1} \mathrm{H}$ NMR $\left(600 \mathrm{MHz}, \mathrm{CDCl}_{3}\right.$ ) of compound $\mathbf{1 1 b}$
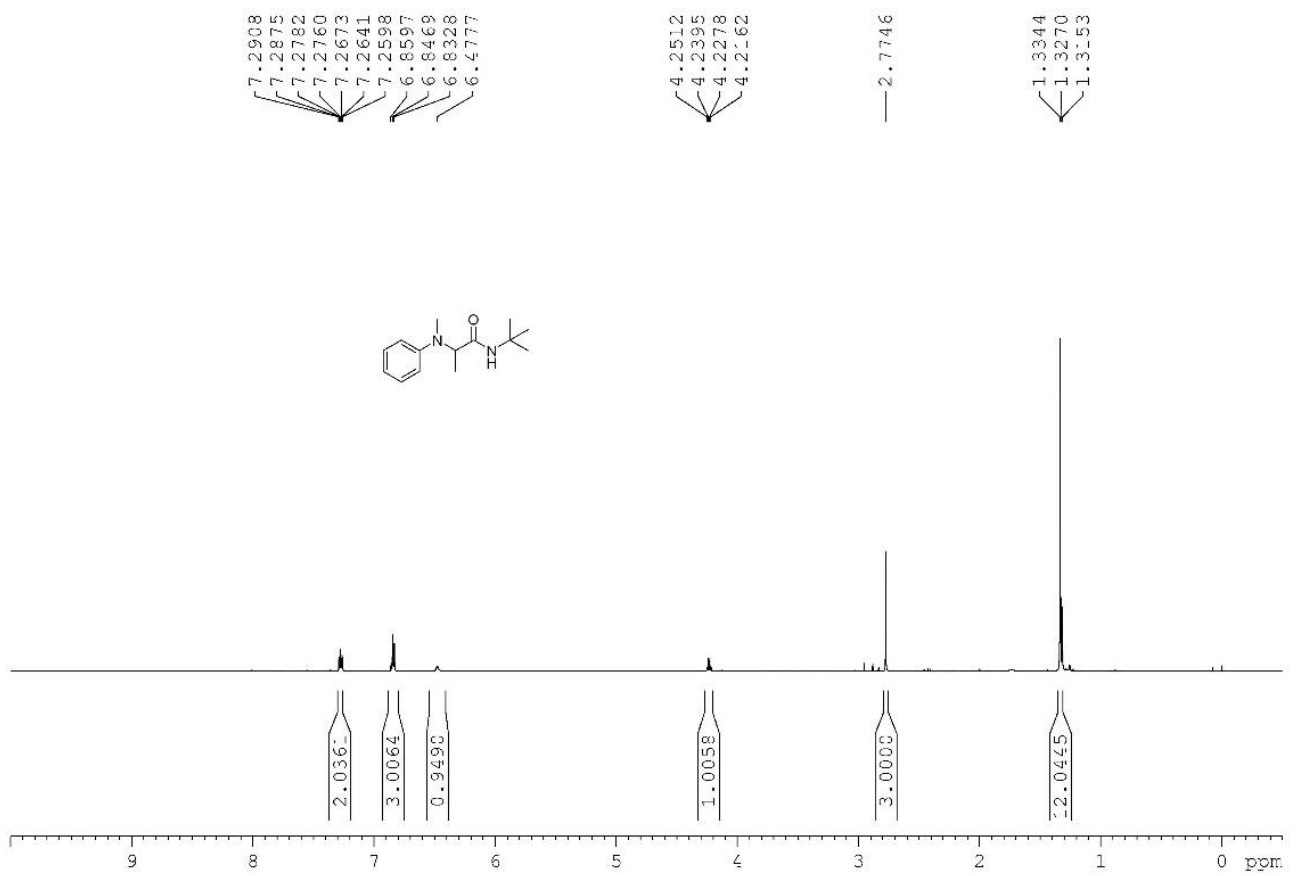
${ }^{13} \mathrm{C}\left\{{ }^{1} \mathrm{H}\right\}$ NMR (151 MHz, $\mathrm{CDCl}_{3}$ ) of compound $\mathbf{1 1 b}$

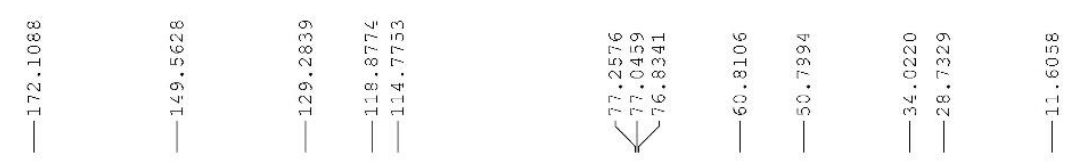

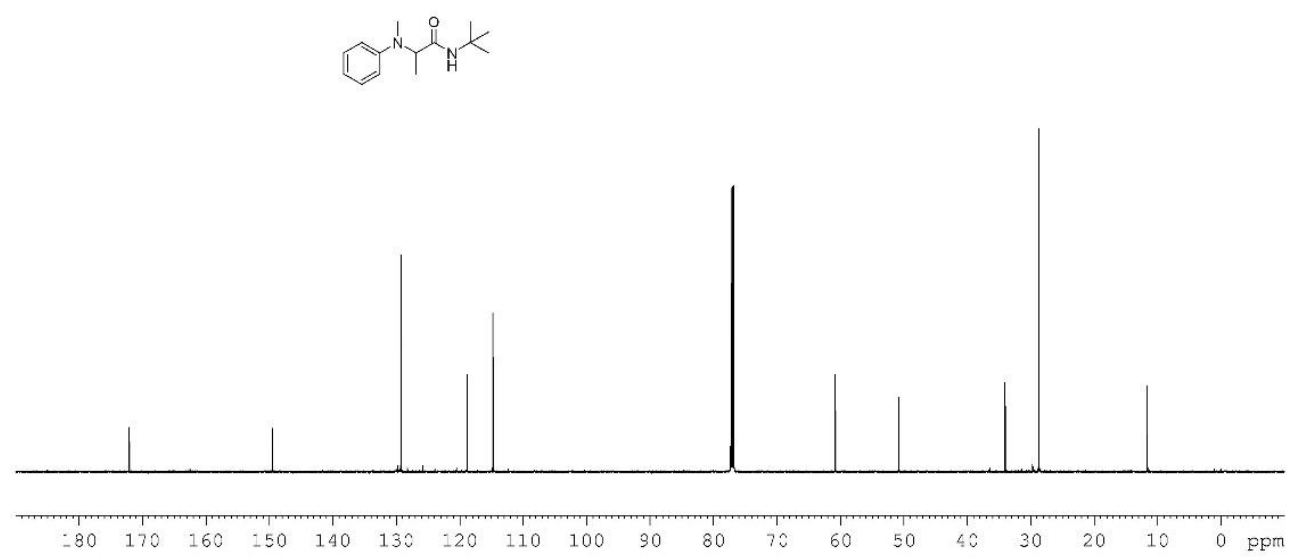

${ }^{1} \mathrm{H}$ NMR (600 MHz, $\mathrm{CDCl}_{3}$ ) of compound 12

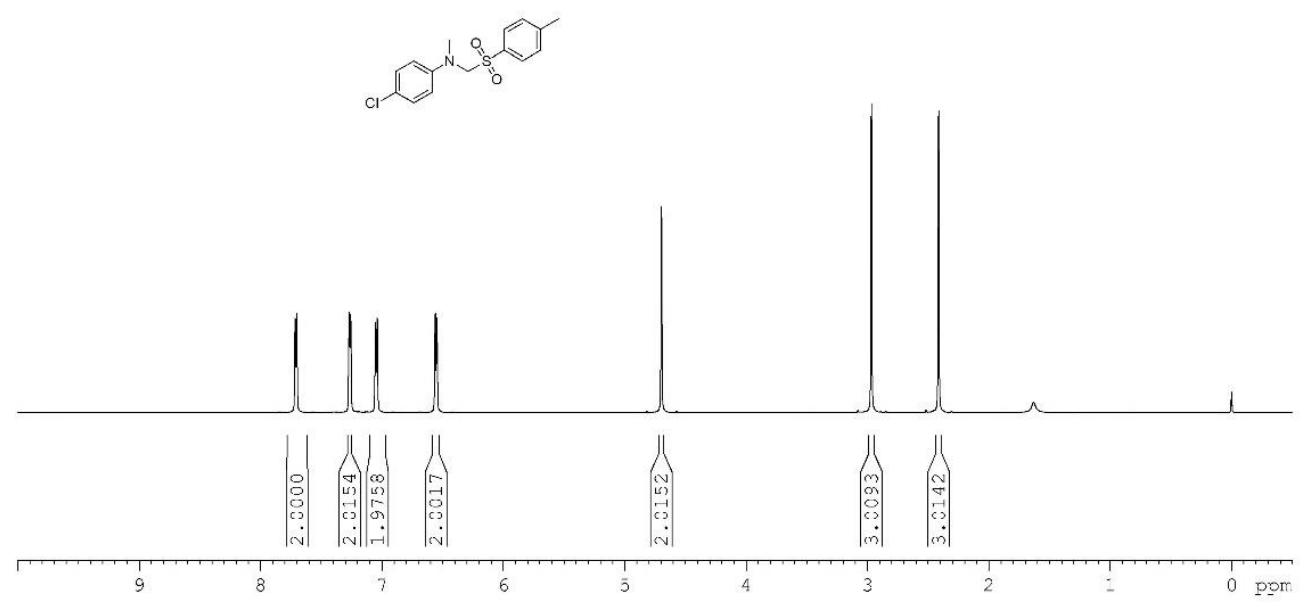


${ }^{13} \mathrm{C}\left\{{ }^{1} \mathrm{H}\right\}$ NMR (151 MHz, $\mathrm{CDCl}_{3}$ ) of compound $\mathbf{1 2}$
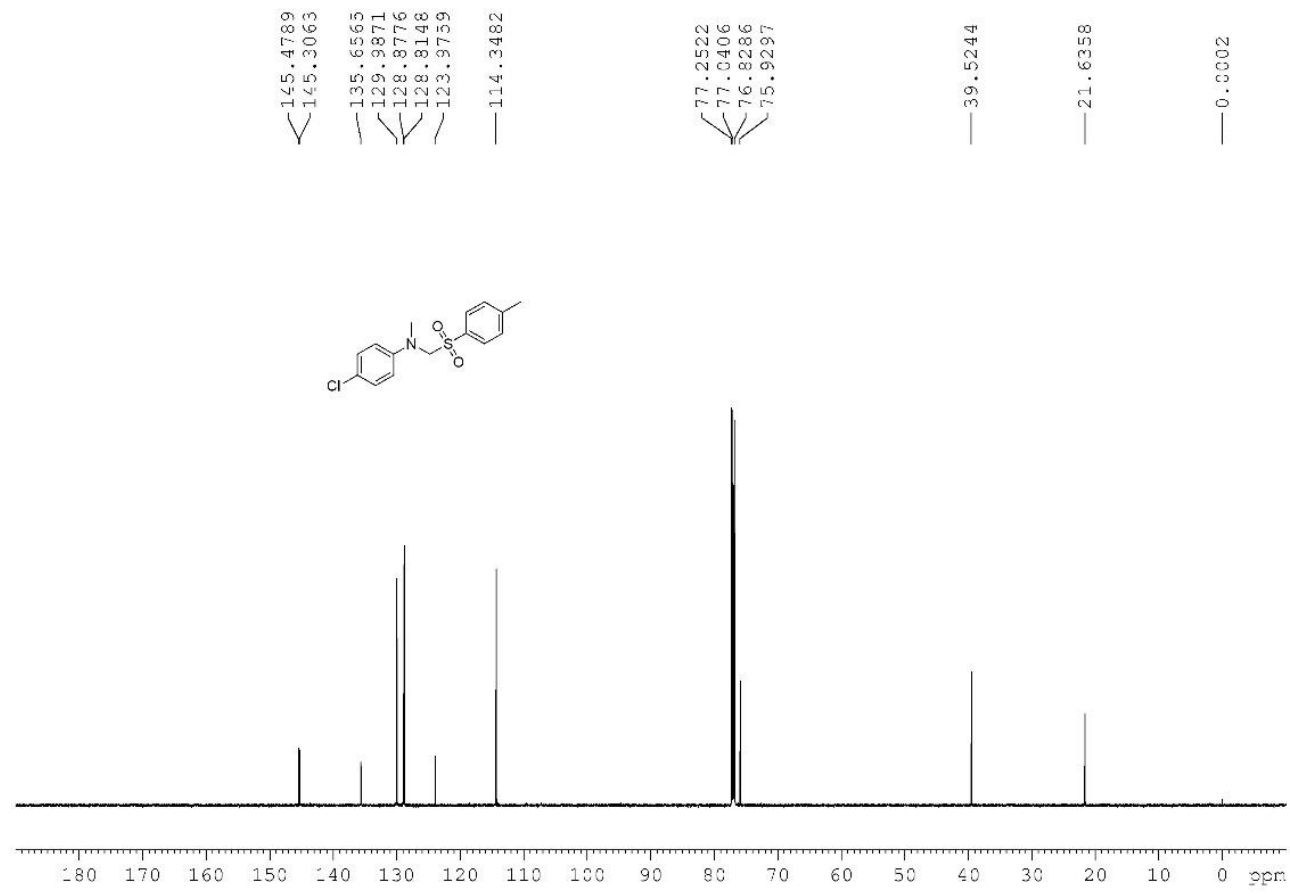

${ }^{1} \mathrm{H}$ NMR $\left(600 \mathrm{MHz}, \mathrm{CDCl}_{3}\right)$ of compound $\mathbf{1 3}$
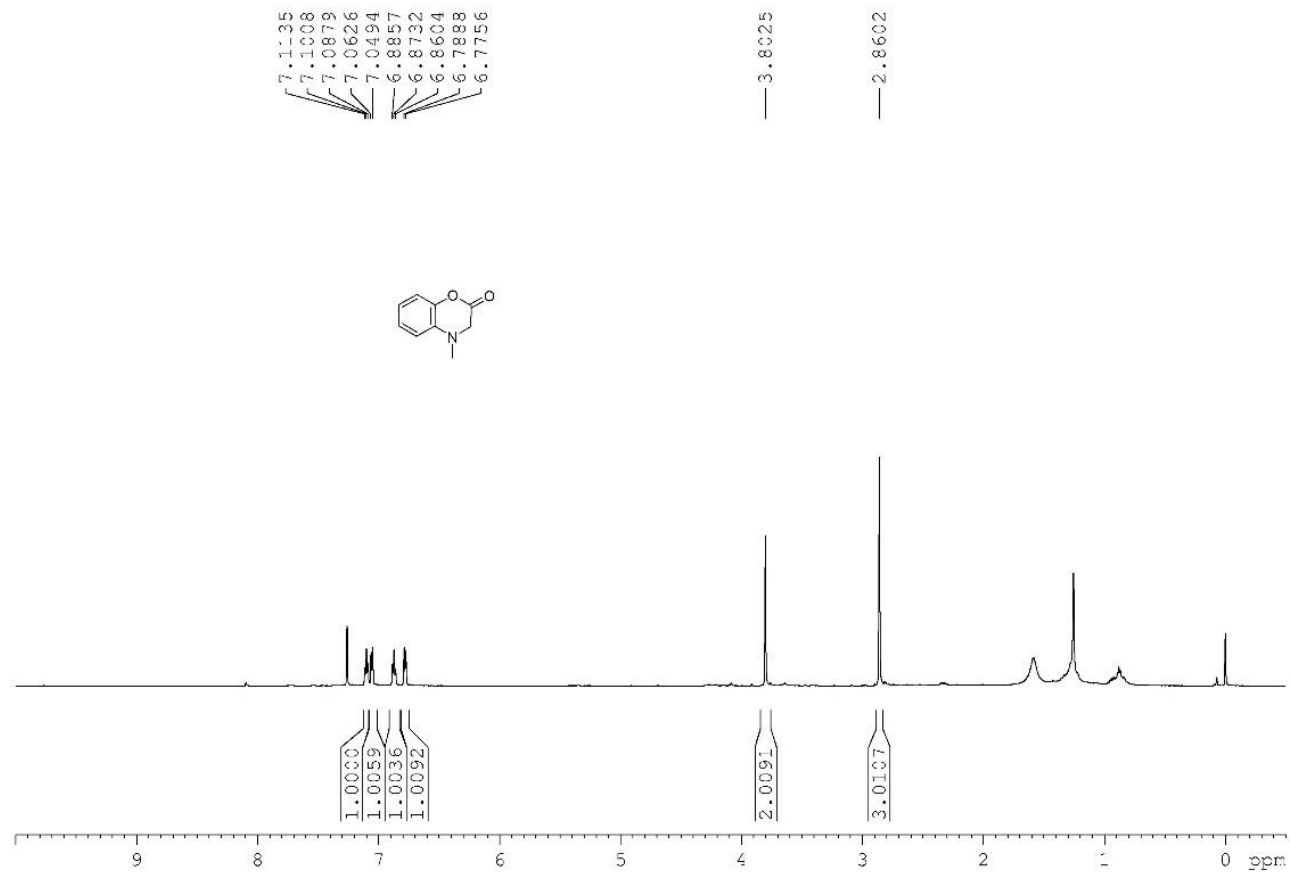
${ }^{13} \mathrm{C}\left\{{ }^{1} \mathrm{H}\right\}$ NMR (151 MHz, $\mathrm{CDCl}_{3}$ ) of compound $\mathbf{1 3}$

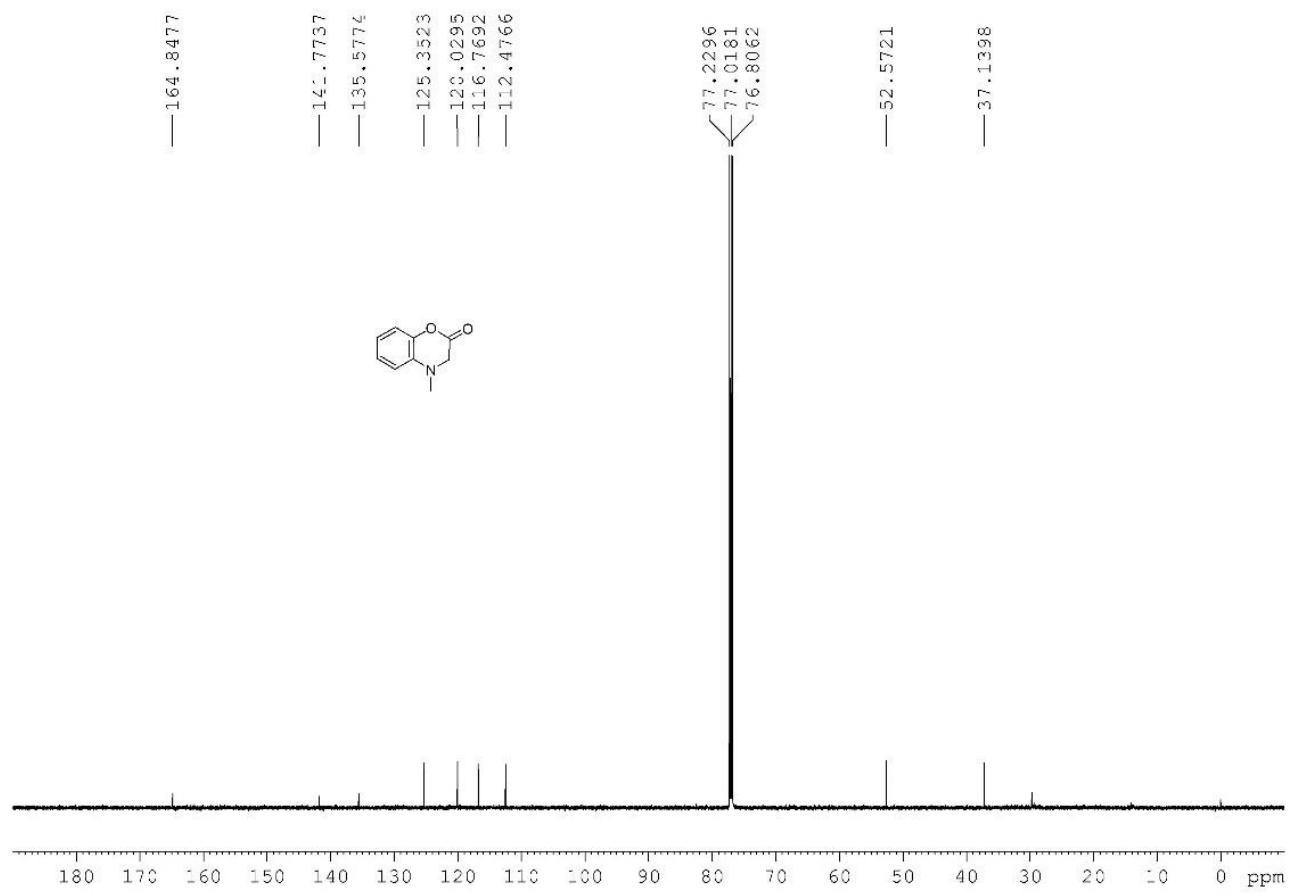

